\title{
Nonparametric consumer and producer analysis
}

\author{
Citation for published version (APA):
}

Houtman, M. (1995). Nonparametric consumer and producer analysis. [Doctoral Thesis, Maastricht University]. Rijksuniversiteit Limburg. https://doi.org/10.26481/dis.19950915mh

Document status and date:

Published: 01/01/1995

DOI:

10.26481/dis.19950915mh

Document Version:

Publisher's PDF, also known as Version of record

\section{Please check the document version of this publication:}

- A submitted manuscript is the version of the article upon submission and before peer-review. There can be important differences between the submitted version and the official published version of record.

People interested in the research are advised to contact the author for the final version of the publication, or visit the DOI to the publisher's website.

- The final author version and the galley proof are versions of the publication after peer review.

- The final published version features the final layout of the paper including the volume, issue and page numbers.

Link to publication

\footnotetext{
General rights rights.

- You may freely distribute the URL identifying the publication in the public portal. please follow below link for the End User Agreement:

www.umlib.nl/taverne-license

Take down policy

If you believe that this document breaches copyright please contact us at:

repository@maastrichtuniversity.nl

providing details and we will investigate your claim.
}

Copyright and moral rights for the publications made accessible in the public portal are retained by the authors and/or other copyright owners and it is a condition of accessing publications that users recognise and abide by the legal requirements associated with these

- Users may download and print one copy of any publication from the public portal for the purpose of private study or research.

- You may not further distribute the material or use it for any profit-making activity or commercial gain

If the publication is distributed under the terms of Article $25 \mathrm{fa}$ of the Dutch Copyright Act, indicated by the "Taverne" license above, 
NONPARAMETRIC CONSUMER AND PRODUCER ANALYSIS 
- 1995 Martijn Houtman, Voorburg

Druk: Datawyse Maastricht / Krips Repro Meppel

CIP-DATA KONINKLIVKE BIBLIOTHEEK, DEN HAAG

Houtman, Martijn

Nonparametric consumer and producer analysis / Martijn Houtman. - [S.1. : s.n.]. - III.

Thesis Rijksuniversiteit Limburg Maastricht. - With index, ref. - With summary in Dutch.

ISBN $90-9008566-1$.

NUGI 681

Subject headings: consumer theory; nomparametric approach 1 producer theory; nonparametric apptoach. 
NONPARAMETRIC CONSUMER AND PRODUCER ANALYSIS

\section{PROEFSCHRIFT}

ter verkrijging van de graad van doctor aan de Rijksuniversiteit Limburg te Maastricht, op gezag van de Rector Magnificus, Prof. mr. M.J. Cohen, volgens het besluit van het College van Dekanen, in het openbaar te verdedigen op

vrijdag 15 september 1995 om 14.00 uur

door

Martijn Houtman

geboren te 's-Gravenhage 
Promotor

Co-promotor

Beoordelingscommissie: Prof. dr. H.J.M. Peters (voorzitter)

Prof. dr. J. Muysken

Prof. dr. H.R. Varian (University of Michigan, USA)

This research was sponsored by the Foundation for Law and Government (Reob), which is part of the Netherlands Organisation for Scientific Research (NWO). 
TO MY FAMILY AND PARENTS 
$\therefore$ 


\section{ACKNOWLEDGEMENTS}

This dissertation is a result of efforts that have been made possible by the support of a number of people. I wish to thank Elmer Sterken and Gerard Kuper for not, complaining about smoking cigars in their neighbounhood and, of course, for their positive support that increased my interest in the economic sciences. Adriaan wan Zon has inspired me while I was writing the computer programs in order to apply the theory to empirical data. Leo R.J. Westeman had the courage to read this dissertation from beginning to end. He has made many useful suggestions and comments. Finally, I wish to thank Gerard Sierksema for his comments on graph theory and Jan C. Willems for reading Part IV, that is concerned with the user cost and volume of capital.

Voorburg, Aprïl 1995 


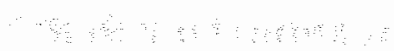

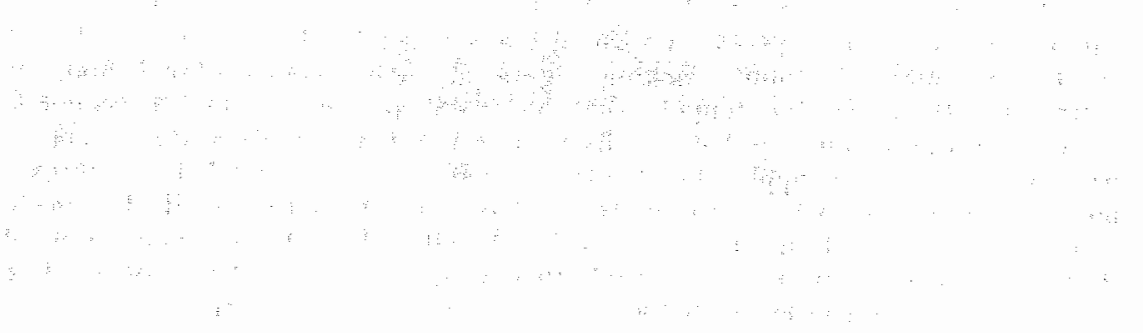

$\vdots$

; 


\section{CONTENTS}

Frequently Used Notation

xiii

Common Notation

Specific Notation

xili

Introduction

Contents

xiv

1

2

\section{PART I CONSUMER DEMAND}

\section{Introduction}

1.1 Neoclassical Theory

1.2 Basis for a Nonparametric Consumer Theory . .

1.3 Differences with Standard Theory

1.4 Application of the Nonparametric Theory 7

1.5 Contents of Part I

\section{Consumer Demand}

2.1 Introduction

2.2 Consumer Demand

2.3 Rationalizing Preference Orders and Utility Functions

2.4 Data Enclosure

2.5 Efficiency Transformations

2.6 Efficiency Upper Bounds

2.7 Existence of a Rationalizing Preference Order

2.8 The Revealed Preference Axiom and Dutch Consumer Data

2.9 Existence of a Rationalizing Utility Function

\section{Limearly Homogeneous Consumer Demand}

3.1 Introduction

3.2 Linearly Homogeneous Utility Functions

3.3 Existence of a Rationalizing Linearly Homogeneous Function

3.4 The Existence Problem in Case of Inefficiency

3.5 A Linearly Homogeneous Function for Dutch Consumer Data

3.6 Restrictions on Linearly Homogeneous Utility Functions

3.7 Construction of Nonparametric Bounds

3.8 Nonparametric Bounds for Dutch Consumer Data

3.9 Approximating Nonparametric Bounds for Concave Functions 39

3.10 Rationalizing Nonparametric Bounds

4.2 Weakly Separable Utility Functions $\quad 42$

4.3 Existence of a Rationalizing Aggregator Function 44

4.4 Existence of a Rationalizing Weakly Separable Function 45

4.5 The Assumption of Strictly Increasing Cost Functions 48

5 Some Nonparametric Theory : 49

5.1 Introduction 49

5.2 Closed Mappings 49

5.3 Approximately Finite Data 51

5.4 Examples of Approximately Finite Data

5.5 Utility Maximization and Cost Minimization : 54

5.6 Utility Functions and Convergence 55

5.7 Existence of a Rationalizing Concave Function 56 
6 Nonparametric Analysis of Aggregate Data

6.1 Introduction

6.2 Empirical Results

6.3 Explanations for the Empirical Results

6.4 The Use of Price Index Numbers

6 Results for Aggregate Dutch Consumption Data 60

6.6 Results for Aggregate Random Data $\quad: \quad: 62$

6.7 Evaluation and Some More Results 63

6.8 Summary 65

\section{PART II PRODUCER DEMAND}

\section{Introduction}

7.1 Theory of Producer Behaviour $\quad 66$

7.2 Producer Demand 66

T.3 Technical Inefficiency and Frontier Production Functions $\quad 66$

7.4 Technical Change

7.5 Rationalizing Production Functions
7.67

7.6 Application to Empirical Data : $\quad 68$

7.7 Contents of Part II 68

8 Producer Demand $\quad 69$

8.1. Introduction $\quad \cdots$

8.2 Producer Demand $\quad 69$

8.3 Frontier Production Functions $\quad \cdots \quad 70$

8.4 Technical Progress $\quad 72$

8.5 Hicks Neutral Change $\quad 74$

8.6 Neutral Change : : $\quad 78$

8.7 Efficiency Transformations for Neutral Change $\quad 81$

9 Linearly Homogeneous Producer Demand $\quad \mathbf{8 3}$

9.1 Introduction $\quad 83$

9.2 Conditions for Linearly Homogeneous Demand 84

9.3 Upper Bounds and Producer Demand $\quad 85$

9.4 Frontier Production Functions 86

9.5 Technical Progress $\quad 89$

9.6 Technical Progress for Frontier Production Functions $\quad 90$

9.7 Hicks Neutral Change 91

9.8 Neutral Change 94

10 Homothetic Producer Demand 99

10.1 Introduction $\quad 99$

10.2 Existence of a Homothetic Production Function 99

10.3 Restrictions on the Underlying Linearly Homogeneous Function : 101

11. Weakly Separable Producer Demand $\quad$ : : : : $\quad 104$

11.1 Introduction $\quad 104$

11.2 Existence of an Aggregator Function , $\quad 104$

11.3 Existence of a Weakly Separable Function 105

11.4 Existence of a Linearly Homogeneous Subfunction 106

11.5 Existence of a Linearly Homogeneous Weakly Separable Function 108

\section{PART III PROFIT MAXIMIZATION}

12.1 Textbook Theory 112

12.2 Profit Maximization 113

$\begin{array}{ll}12.3 \text { Contents of Part III } & 114\end{array}$ 
13.1 Introduction

13.2 Conditions for Profit Maximization

13.3 Bounds for Profit Maximization

13.4 Frontier Production Functions

13.5 Technical Progress

13.6 Hicks Neutral Change

120

13.7 Neutral Change

121

124

14 Homothetic Profit Maximization

14.2 Existence of a Homothetic Production Function

15 Weakly Separable Profit Maximization

132

15.1 Introduction

132

15.2 An Aggregator Function

132

15.2 Existence of a Weakly Separable Function

139

\section{PART IV CAPITAL}

\section{Introduction}

136

16.1 Static or Dynamic Producer Behaviour

16.2 The Dynamic Optimization Problem

136

136

138

16.4 Capital Stocks

1.40

16.5 Contents of Part IV

141

141

141

143

144

145

147

148

17.7 Summary and Conclusions

150

150

150

151

152

154

156

156

157

159

160

19.5 The Discrete-Time Model

Generalizations of the Nonparametric Theory

Applications of the Nonparametric Theory 


\section{APPENDIX}

A Binary Relations $\quad 165$

A.1 Binary Relations 165

A.2 Basic Properties of Binary Relations 165

A.3 Particular Binary Relations 165

A.4 Extensions of Orders $\quad 166$

A.5 Extensions of Binary Relations 167

B Graph Theory 169

B.1 Introduction 169

B.2 Graphs $\quad 169$

B.3 Subgraphs $\quad 170$

B.4 Paths in a Graph

B.5 Strongly Connected Vertices $\quad 170$

B.6 Absorption of a Vertex 171

B.7 Feedback Vertex Sets $\quad 171$

C Path Algebra's and Labelled Graphs $\quad 173$

C.1 Introduction $\quad 173$

C.2 Path Algebra $\quad \therefore \quad \therefore \quad 173$

C.3 Examples of Path Algebra's 174

C.4 Labelled Graphs $\quad 174$

$\begin{array}{ll}\text { C.5 The Weak Closure of an Adjacency Matrix } & 175\end{array}$

C.6 Theorems for Labelled Graphs 176

D Laplace Transforms $\quad 180$

E Description of the Dutch Industry Data $\quad 181$

E.1 Prices and Quantities 181

E.2 Capital Stocks 182

E.3 Sensitivity to the Choice of Initial Values 183

E.4 User Cost of Capital $\quad 183$

F Results of Producer Tests $\quad \therefore \quad 186$

G Overview of Nonparametric Tests 188

G.1. Consumer Demand $\quad 188$

G.2 Producer Demand $\quad 189$

G.3 Profit Maximization $\quad 191$

References $\quad: \quad 193$

$\begin{array}{ll}\text { Related Literature } & 197\end{array}$

Index 201

Nederlandse Samenvatting / Summary in Dutch 206

Generalisatie van de niet-parametrische theorie $\quad 207$

Toepassingen van de niet-parametrische theorie : $\quad 207$

$\begin{array}{lr}\text { Curriculum Vitae } & 200\end{array}$ 


\section{FREQUENTLY USED NOTATION}

\section{Common Notation}

The notation of, for instance,

$$
f(x)=c \quad(x \in X)
$$

means $f(x)=c$ for all $x \in X$.

For preorders Herstein and Milnor's (1953) notation is used. In that case a preorder is denoted as $\succsim$, using the notations:

$$
\begin{aligned}
& x \sim y \Leftrightarrow x \succsim y \text { and } y \succeq x, \\
& x \succ y \Leftrightarrow x \succsim y \text { and not } y \succsim x, \\
& x \precsim y \Leftrightarrow y \succsim x, \\
& x \prec y \Leftrightarrow y \succ x .
\end{aligned}
$$

These relations have usually the following interpretation:

$x \succsim y: y$ is not preferred to $x$,

$x \succ y: x$ is preferred to $y$,

$x \sim y: x$ is indifferent to $y$.

In the text such a preorder $\succsim$ is denoted as $\succ, \sim$ for typographical convenience.

Next, the following common notation is used.

$\boldsymbol{A}, \boldsymbol{B}$ etc. operatior

$A, B$ etc. matrix, set, binary relation

0 vector of zero's

$\emptyset$ empty set

$\approx$ approximately equal

$\equiv$ is equal by definition

* convolution:

$$
(f * g)(t) \equiv \int f(t-\tau) g(\tau) d \tau
$$

a function of a function: $f \circ g(x) \equiv f(g(x))$

$\mathbb{R}$ real numbers

$R_{\text {, nonnegative real numbers }}$

$\mathbb{R}_{++}$positive reall numbers

C complex numbers

N positive integers

2 integers

$P^{c}$ complement of a set $P$

$R^{*}$ transitive closure when $R$ is a binary relation

$C^{*}$ shortest path matrix when $C$ is a cost matrix for paths

$\mathcal{L}$ luaplace transformation:

$$
\mathcal{L}[f] \equiv \int f(\tau) \mathrm{e}^{8 \tau} d \tau
$$

$\tilde{g}$ Laplace transform of $g: \tilde{g} \equiv \mathcal{L}[g]$

$\breve{g}$ transformation of function $g$ such that: $\breve{g}(t) \equiv g(-t)$

$|x|$ absolute value of $x$

$\|x\|$ Euclidean length of vector $x$ 
$x$ deriwative

$$
x(t)=\frac{d}{d t} x(t)
$$

$\langle x, y\rangle$ innerproduct of $x$ and $y$

$A^{t}$ transposed matrix $A$

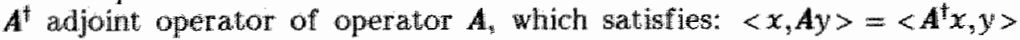

$\delta(\cdot)$ Delta function:

$$
\int f(\tau) \delta(\tau) d \tau \equiv f(0)
$$

$1(\cdot)$ step function:

$$
U(t)= \begin{cases}0 & (t<0) \\ 1 & (t \geq 0)\end{cases}
$$

\section{Specific Notation}

Concerning sets the following specific notation is used:

$$
\begin{aligned}
& \mathcal{R} \text { set of preference relations } \\
& \mathcal{F} \text { set of functions } \\
& \mathcal{D} \text { set of data }
\end{aligned}
$$

To denote that we consider consistency with a certain type of economic behaviour, the lower indices $c, u, d$, and $p$ are used:

$\mathcal{F}_{c}$ the set of functions consistent with cost minimization

$\mathcal{F}_{u}$ the set of functions consistent with utility maximization

$\mathcal{F}_{d}$ the set of functions consistent with demand

$\mathcal{F}_{p}$ the set of functions consistent with profit maximization

Concerning $D$ and $R$ these indices have an analogous meaning. Furthermore, the indices are used in combination with the following upper indices:

$\mathcal{F}^{o}$ set of functions for data that includes observations of output

$\mathcal{F}^{f}$ set of frontier production functions

Special functions, that have as argument a set $F$ of functions $f: X \rightarrow \mathbb{R}$, are:

$$
\begin{aligned}
& U(F, x) \quad=\sup _{f \in F} f(x) \quad \text { upper bound } \\
& U_{/}\left(F, x, x^{\prime}\right) \equiv \sup _{f \in F} f(x) / f\left(x^{\prime}\right) \text { relative upper bound } \\
& U_{-}\left(F, x, x^{*}\right) \equiv \sup _{f \in F} f(x)-f\left(x^{*}\right) \text { difference upper bound } \\
& L(F, x) \quad \inf _{\mathbb{f} \in F} f(x) \quad \text { lower bound } \\
& L_{/}\left(F, x, x^{\prime}\right) \equiv \inf _{f \in F} f(x) / f\left(x^{\prime \prime}\right) \text { relative lower bound } \\
& L_{\ldots}\left(F, x, x^{\prime}\right) \equiv \inf f(x)-f\left(x^{\prime}\right) \text { difference lower bound } \\
& f \in F^{2}
\end{aligned}
$$




\section{INTRODUCTION}

This book contains both a theoretical and empirical research of nomparametric consumer and producer theory. The emphasis lies on the theoretical development of a nonparametric theory, while the empirical research is meant as an example of application of the developed theony to real data.

The starting-point of the nonparametric theory is a model of optimizing behaviour that satisfies a certain symmetry. This idea has applications in different fields of science. One is the field of nonparametric statistics, where the symmetry assumption is applied to probability distributions. Another field where results are derived by using symmetry assumptions is physics. The role of symmetry is very important in classical mechanics and quantum physics. In classical mechanics the Noether theorem is used to derive conservation laws in case the dynamical system has a symmetric propery. For example, the conservation of energy, momentum, and angular momentum follows from the symmetry assumption that the Lagrangian is invariant under time translation, space translation, and rotation respectively. In quantum field theory the assumption that the dynamics are invariant for Poincare transformations is a starting-point to derive important theoretical results. It is clear that this fruitful nomparametric approach in physics may become important in economics. In this book such an approach is not applied to dynamical optimization problems, but to series of statical optimization.

The usual approach to analyse a certain specified type of ecomomic behaviour is the specification of a production or utility function after which the corresponding economic behaviour is analysed. This book stresses the importance of a reverse approach that is especially suitable for empirical applications. This reverse approach considers data of observed behaviour and analyses properties of the corresponding set of possible utility or production functions. This approach relies on the assumption of a symmetry in the optimization model.

When we consider data that might be generated by statical optimization, the following questions are appropriate for the set of possible utility or production functions, which correspond with such an optimization model:

1. Is there such a function?

2. What may be the properties of such a function?

3. Are there restrictions on such functions?

The subject of this research is divided in consumer demand, producer demand and profit maximization. Concerning these kinds of optimization behaviour, the existence of a suitable optimized function is considered. Furthermore, several properties of these functions are considered: limear homogeneity, homotheticity and weak separability. Hesides utility functions, the theory of consumer demand also treats preference orders.

A fourth subject of research is capital. Using capital in production functions is easy in textbook theory, but a real problem in empirical studies. When the nonparametric producer theory was ready to be applied to data, I discovered - after solving the problem of lacking price data - that I missed prices and quantities for a simple variable in the nonparametric theory: capital. When I tried to construct these prices and corresponding quantities, I was surprised by the usual rough approach to this problen in empirical production models. In these models investments are nearly allways 
exponentially decaying. This hypothesis is of course not based on empirical evidence, but on easy solutions for the capital problem. Exponential decay allows using a simple dynanical model for the capital stock. For this model the user cost of capital can easily be solved by using the maximum principle of Pontryagin (1962). Except for the lack of a general theory for the user cost of capital, using unreliable capital data may be a reason for this attitude. In case of unireliable data an error more or less does not matter. Another problem is the question whether producers are behaving as rational optimizers.

I, used to fairly exact measurements in physical experiments, was at first not aware of these problems in economics. So I found it necessary to develop a rigorous theory, suitable for reliable capital data and rational optimizing producers. The basic ideas that are used in this book to derive a user cost of capital theory are inspired by the theory of quantum physics and they may be new in the context of a capital theory.

\section{Contents}

The treatment of consumer demand in Part $I$ is based on a definition of consumer behaviour. This definition differs in several ways from the neoclassical textbook definition of utility maximization. In the neoclassical theory one often uses restrictions on the utility function to assure that a utility maximizer is a cost minimizer. Here I assume a priori that a consumer is both a utiluty maximizer and a cost minimizer.

Another difference with the standard theory is the allowance of economica] inefficiency as is also proposed by Afriat (1973). The idea of Afriat is generalized by using efficiency transformations. The idea behind such a transformation is simple. Efficiency transformations are used to transform data into new data with the aim to relax the conditions for consumer demand. Such transformations are dependent on an efficiency parameter that indicates how seriously we have deformed the data.

Furthermore, I do not use prices, but functions to describe the cost of a quantity vector. This allows for the application of the theory to noncompetitive markets. Using nonlinear budget constraints in revealed preference theory is not new. Richter (1966) used the concept of budget sets as a generallization of the usual linear budget constraint.

In Part II the jdea of consumer demand is applied to producer demand, where the utility function is replaced by a production function. In contrast to consumer demand, a producer demand theory includes the observation of produced output. So, the theory of producer demand can be conceived as a consumer demand theory in which output may be observed. Moreover, a producer demand theory may consider frontier production functions, technical progress and technical inefficiency.

Part III applies the ideas that are used in Part I and Part II, like efficiency transformations and using cost functions instead of prices, to profit maximization.

Part IV treats the essential problem of capital: the dynamical relation of investments and capital and the corresponding dynamical optimization problem for a producer. The point of view in this part of the book differs from the other parts. While Part I, II and III studies mainly the properties of data, derived from consumer and producer behaviour, the main subject in Part IV, however, is the development of a behaviour model itself. This part studies how one may derive prices and quantities for capital, given a dymamic capital investment relation. 
The user cost of capital is analysed, using the conventional assumption that investment costs are linear. There is a connection with the nonparametric approach, because the theory does not depart from a parametric specified capital investment relation. This relation is specified by using a few general assumptions.

Another main subject in Part IV is the generation of capital stocks To generate these stocks a simple parametric model is proposed. This model is derived from the statistical properties for the lifetime of investment. It is argued that the parametric model is flexible enough to fit with empirical survival functions. 


\section{PART I \\ CONSUMER DEMAND}

\section{INTRODUCTION}

\subsection{Neoclassical Theory}

The neoclassical theory postulates that a consumer maximizes his utility subject to a budget constraint. This is formalized as follows. Let $f(\cdot)$ be the consurner's utility function, representing his preferences, and $p$ the vector of prevailing prices. The choice behaviour of a utility maximizing consumer is now

$$
f\left(x^{5}\right)=\sup _{p x \leq p x^{*}} f(x)_{m}
$$

A simple example of this form of consumer behaviour, which is usually presented in text books, is illustrated in Figure 1.1.

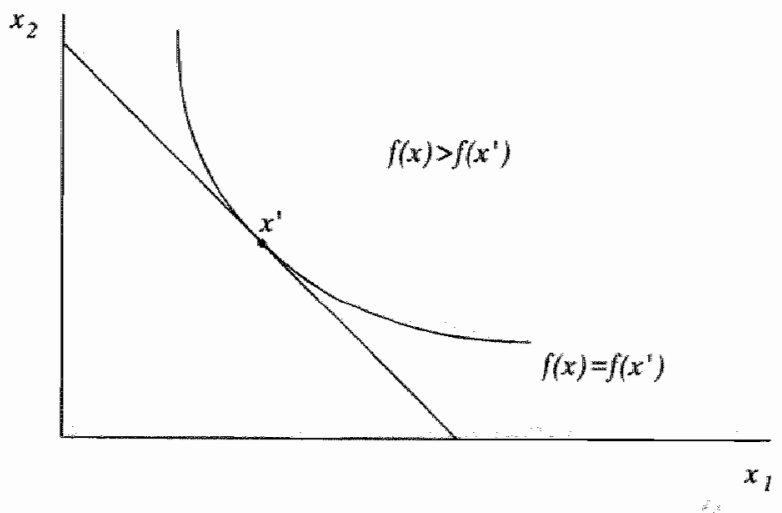

Figure 1.1 Consumer Demand

The assumption of utility maximization, however, does not guarantee that the consumer spends his budget without a waste of money, A utility maximizing consumer may waste money when there are 'thick' indifference curves, as is illustrated in Figure 1.2. A cost minimizing consumer, who does not waste money, displays the behaviour

$$
p x^{*}=\inf _{f(x) \geq f\left(x^{\prime}\right)} p x
$$


To exclude "thick" indifference curves, one often assumes that consumers utility function $f$ is a locally nonsatiated function. For a utility maximizing consumer this assumption assures that he spends his entire budget $p x^{\prime}$, while being a cost minimizer.

Definition: Suppose $f: X \rightarrow \mathbb{R}$ and $x^{*} \in X$. Then $f$ is said to be locally nonsatiated at $x^{\prime}$ if for every open neighbourhood $F$ of $x^{2}$ there is a point $x \in E^{*}$ such that $f(x)>f(x)$. If $f$ is locally nonsatiated at every point of $X$, then $f$ is said to be locally nonsatiated.

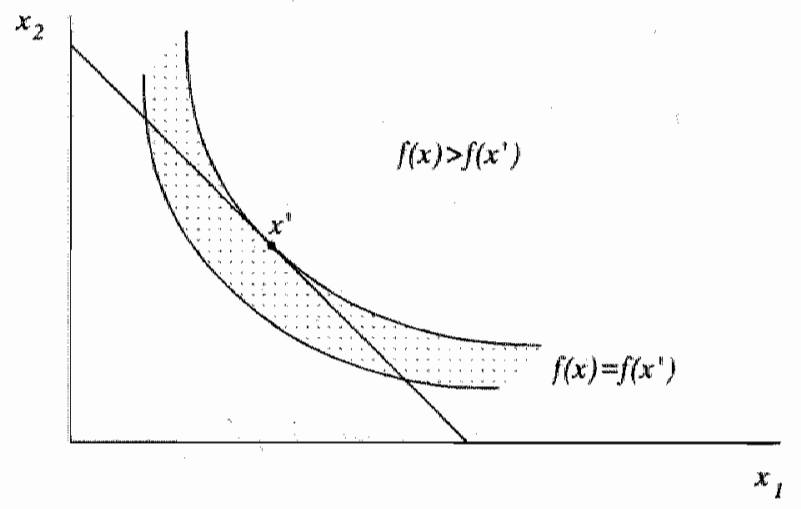

Figure 1.2 Thick Indifference Curve

We can generalize the above theory by replacement of the utillty function by a preorder. The choice behaviour for such a preorder, called a preference order, is derived in the same way as for a utility function. For instance, the inequality $f\left(x^{*}\right) \geq f(x)$, for a utility function $f$, means that the choice $x$ is not preferred to $x^{\prime}$. Similarly, for a preorder $\succ, \sim$ this is the case when one has $x^{\prime} \succsim x$.

\subsection{Basis for a Nonparametric Consumer Theory}

This part is concemed with the nompanametric analysis of consumer behaviour. The idea of such a nonparametric analysis is as follows. Suppose that one observes data of consumer behaviour. Then one may postulate that this data is generated by a rational consumer, using a preference order or utility function. Furthermore, one may postulate the hypothesis that this preference order or utility function is member of a certain nonparametric described family of preorders or functions respectivelly. Examples of such nonparametric families are families of functions that satisfy a certain symmetry, i.e. they are invariant under certain transformations. For example, a widelly used nomparametric family of functions is the family of linearly homogeneous functions. This family can be described as the family of functions $f(x)$ that is invariant under transformations $T_{\lambda}$ of the form

$$
\left(T_{\lambda} f\right)(x)=\lambda^{-1} f(\lambda x) \quad(\lambda \neq 0) .
$$

Another family is the family of weakly separable functions $f(x)$ of the form 
$f\left(x_{1}, x_{2}\right)=g\left(x_{1}, h\left(x_{2}\right)\right)$, where $g$ is increasing in $h$. Such a function is invariant under the transformation $T_{h}$ of the form

$$
\left(T_{h} f\right)\left(x_{1}, x_{2}\right)=\inf _{h\left(x_{2}\right) \geq h\left(x_{2}\right)} f\left(x_{1}, x_{2}^{\prime}\right) \text {. }
$$

So symmetry properties can be used to define a nomparametric family of functions.

In the nonparametric consumer analysis there are now two central problems. First, there is the question which properties have to be satisfied by the given data set in order to accept a postulated hypotheses. Such a property may be a symmetry that has to be satisfied by the data. Secondly, there is the question what the given data set tells us about the underlying preference order or utility function, for instance, in the form of restrictions on the utility function. Such restrictions can be seen as conserved properties for the consumer data, if they remain valid when new consistent data are added.

\subsection{Differences with Standard Theory}

The starting-point in this book is a generalization of the standard neoclassical theory. This generalization differs from the standard neoclassical theory in three ways:

1. In the standard theory a consumer is a utility maximizer. In this book, however, we assume a priori that a consumer is both a utility maximizer and a cost minimizer.

2. The standard assumption of given prices is generalized. It is assumed that the consumer has a given function that describes the cost of his choices. I will call such a function a cost function.

3. Economical inefficiency is allowed.

The assumption that a consumer is both a utility maximizer and a cost minimizer is advantageous, because one can skip the functional restrictions, like nonsatiation, needed to assure that the consumer is also a cost minimizer.

The second difference with the standard theory, the use of cost functions, does not cause theoretical complications. It may even help to simplify the theory. This generalization means that the analysis applies not only to competitive consumers, where prices are fixed, but also to noncompetitive consumers, where prices are a function of consumer demand.

The nonparametric approach is not very popular in the current literature. This has to do with the limited empirical use of this approach in case one supposes consumer and producer behaviour is efficient. In empirical studies the assumption of efficient consumer behaviour is often violated. In order to apply the nonparametric methods, I will introduce efficiency measures that indicate how serious such a violation is. Hence, the allowance of economical inefficiency has an important pragmatic reason: it may be of great help in empirical applications. There is however a drawback of the inclusion of economical inefficiency in the theory, It may cause complications, which interfere with the need for a simple clear theory, So, I had the choice between the presentation of a nice clean theory, concerning efficient economical behaviour, or to accept some complications due to the inclusion of economical inefficiency. I have chosen for the latter, because such a theory is more suitable for empirical applications. 


\subsection{Application of the Nomparametric Theory}

To illustrate the use of the nomparametric theory, developed in this book, the theory is applied to Dutch empirical constimption data. The Netherlands Central Bureau of Statistics (1981) published annual private consumption expenditure data and several types of price index numbers, covering the period 1951-1977, on various levels of aggregation. In this study the geometric price index numbers for 106 commodity groups are used (Netherlands Central Bureau of Statistics, 1981, Table 4). Implicit per capita quantity measures for each commodity group are obtained by the diwision of the nominal commodity group expenditures per capita by the corresponding geometric price index numbers. The data used for this computation, are the expenditure shares for the 106 commodity groups, total expenditures and population data (Netherlands Central Burean of Statistics, 1981, Table 3, 44 and 47).

\subsection{Contents of Part I}

Chapter 2 treats the problem whether there is a preference relation or utility function for given consumer data. The search for a utility function which fits with given consumer demand, the 'Integrability Problem', has been traced as far back as Antonelli (1886). There is however one difference between the 'Integrability Problem' and the problem in Chapter 2. In the 'Integrability Problem' it is assumed that all possible observations of consumer demand are known. However, in this book it is assumed that only certain observations of consumer demand are available, as is the case in an empirical context. This demands a global analysis, based on the axiom of revealled preference in the work of Samuelson $(1948,1950 \%$, Houthakker (1950) and Uzawa (1960), whereas the "Integrability Problem" is solved with a local analysis.

Chapter 3 investigates the existence of linearly homogeneous utility functions for a given data set. This problem is solved for neoclassical consumer behaviour in Afriat $(1972,1981)$, Diewert (1973) and Varian (1983). Their results are generalized to the more general definition of consumer demand used in this book. Another point of interest is the existence of lower and upper bounds for the linearly homogeneous utility function for given consumer demand data. This allows one to derive an idea of consumers utility function without the conventional parametric specification of this function and an estimation of the parameters.

In Chapter 4 the weak separability of utility functions is treated. This problem has not got much attention in the nonparametric approach. Diewert and Parkan (1978, 1985) and Varian (1983) consider concave weakly separable utility functions. In that case one can obtain results by using separating hyperplanes. This book presents new results for consumer demand with no other restriction on the utility function than weak separability.

When I started this research as mathematician between economists, I tried to get some backup from the mathematical department. When I explained the subject to a mathematician there, he said: "I can't help you. This is no mathematics?" Well, Chapter 5 is an attempt to falsify this staternent. It is a mathematical exercise of a nonparametric approach to consumer demand..

In Chapter 6 it is shown that aggregation of random data may result in data that display economical behaviour. This result is obtained by the application of several nonparametric tests to aggregate random data. I have to admit that this part of the research is not a result of a sharp mind. As many results in science it is a result of a mistake. Accidentally a programming mistake did transpose the price matrix in the computer program that evaluated the 
corresponding quantities. When $\mathbb{I}$ discovered this mistake I was surprised about the nice empirical results, which can shortly be described as "Garbage In Economics: Out". Of course this may also happen, when one uses unrellable data. Let this be arning to empirical researchers! 


\section{CONSUMER DEMAND}

\subsection{Introduction}

This chapter contains basic definitions and theorems concerning the nonparametric analysis of consumer behaviour. The starting-point of these theorems is the assumption that there is one and the same utility function for all observed data. Assuming the data refer to observations at different points of time, this means in terms of symmetry that the utility function in the model is invariant under time translation. Using this assumption it will be shown that the axiom of revealed preference is a necessary and sufficient: condition for the existence of a rationalizing preference order. Moreover, as was shown earlier by Afriat (1973), the axiom of revealed preference appears also to be a necessary and sufficient condition for the existence of a rationalizing utility function, provided the consumer data consist of a finite number of elements. That the axiom of revealed preference is not sufficient for infinite data sets follows from counter examples, for example in Chipman et al. (1971). The existence of rationalizing utility functions for data sets of an infinite number of elements is not treated in this chapter. I will consider this problem, which is mainly of theoretical interest, later on.

Important in this chapter is the introduction of efficiency transformations. A feature of an efficiency transformation is the possibility to relax the conditions belonging to efficient behaviour. The idea can be applied to every nomparametric test described in this book. Furthermore, it provides a measure that describes how seriously a nonparametric test is violated.

\subsection{Consumer Demand}

Let us consider an arbitrary choice space $X$ of elements, avalable to a consumer to a certain cost. We may represent the cost of consumers choice $x$ in $X$ by using a function. I will call such a function a cost function. Assuming that costs are nonnegative, we can describe cost functions as follows.

Definition: Let $X$ be a given set. A cost function defined on $X$ is $a$ function $c: X \rightarrow \mathbb{R}_{+}$. The set of all possible cost functions $c: X \rightarrow \mathbb{R}_{+}$is denoted as $C(X)$.

The cost functions $c$ that appear in textbook theory, are simply of the form $c(x)=p x$, where $x$ is a quantity vector and $p$ a given price vector. When consider this particular case, I will suppose prices positive and quantities nonnegatiwe, i.e. $p \in \mathbb{R}_{+++}^{n}$ and $x \in \mathbb{R}_{+*}^{n *}$. To refer to the function $c(x)=p x$ in formulae, I will use the abuse of notation $p$ when actually the function $c$ is meant.

Let us assume that consumer behaviour can be derived from a utility function $f: X \rightarrow \mathbb{R}$. Then a utility maximizer will choose the best $x \in X$ for its money, i.e., given a cost function $c: X \rightarrow \mathbb{R}_{+,}$, his choice behaviour can be described as

$$
f\left(x^{\prime}\right)=\sup _{c(x) \leq c(x)} f(x)
$$

Similarly, a cost minimizer will choose the cheapest $x \in X$ for its utility value, thus 


$$
c\left(x^{\prime}\right)=\inf _{f(x) \geq f\left(x^{*}\right)} c(x) .
$$

The preferences of a consumer for a given utility function can be represented as a weak order of the following form.

Definition: Let $f: X \rightarrow \mathbb{R}$ be a function. Then the weak order $\sim_{*} \sim$ on $X$ induced by $f$ is defined as

$$
x \geq y \Leftrightarrow f(x) \geq f(y) \quad(x, y \in X) .
$$

(Note that preorders $\succsim$ are often denoted as $>, \sim$ in this book, which is not a standard notation).

For the contents of a consumer theory there is no difference in using a weak order or a utility function. We may generalize this theory by using a preorder instead of a weak order. This is a generalization, because any weak order is preorder, but not every preorder is a weak order.

Definition: Let $R$ be a binary relation on $X$. Such a relation may have the following properties. It may be:

- transitive if $x R y$ and $y R z$ implies $x R z$, for every $x, y, z \in X$;

- reflexive if $x R x$ for all $x \in X$;

- strongly complete if $x R y$ or $x R y$ for every $x, y \in X$.

A prearder is a transitive reflexive binary relation. A weak order is a transitive strongly complete binary relation.

One may expect that preferences do order things in a consistent way. So the choice of a transitive binary relation to represent preferences is a natural one. Furthermore, something is as good as itself. Thus preferences are reflexive. So in general we may represent consumers preferences as a preorder. In the special case that a consumer can compare every pair of choices such a preorder is a weak order.

For preference orders we obtain the following definition for a utility maximizer, who chooses the best element in the budget set.

Definition: Let $\succ, \sim$ be a preorder defined on $X$. Then utility maximizing behaviour is defined as

$$
D_{u}(\succsim) \equiv\left\{\left(c, x^{\prime}\right) \in C(X) \times X^{\prime} \mid c(x) \leq c\left(x^{\prime}\right) \Rightarrow x \lesssim x^{\prime} \quad(x \in X)\right\}
$$

Similarly, a cost minimizer chooses the cheapest element in the set of choices of higher or equal utility. Cost mimimizing behaviour is defined as

$$
D_{c}(\succsim)=\left\{\left(c, x^{x}\right) \in C(X) \times X \| x \succsim x^{\prime} \Rightarrow c(x) \geq c\left(x^{*}\right) \quad(x \in X)\right\}
$$

Combining both type of behaviour results in consumer demand, which is defined as $\mathcal{D}_{d}(\succsim) \equiv \mathcal{D}_{u}(\succsim) \cap \mathcal{D}_{\mathrm{c}}(\succsim)$

So, if we write $f$ when we actually mean the weak order induced by $f$, the set of consumer demand $\mathcal{D}_{d}(f)$ contains data $\left(c, x^{\prime}\right)$. such that one has both

$$
\begin{aligned}
& f(x)=\sup _{c(x) \operatorname{sc}\left(x^{\prime}\right)} f(x), \\
& c\left(x^{\prime}\right)=\inf _{f(x) \geq f\left(x^{\prime}\right)} c(x) .
\end{aligned}
$$

Consumer demand is clearly invariant under monotonic transformations of the utility function, i.e. one has $D_{d}(f)=D_{d}(m \circ f)$, where $m$ is a strictly increasing function. In physics such a symmetry is said to be a gauge symmetry. Gauge symmetry means that the behaviour of physical variables can be described by several internally different models, More precisely, a physical model has a gauge symmetry when the observable physical variables are invariant under a transformation of the model equations. 
Now, we have given a definition of consumer demand. What is the use of this definition? A typical empirical situation is the following. We observe a set of consumer data $D=\left\{\left(c_{i}, x^{i}\right)\right\}_{i \in I}$, where $\left(c_{i}, x^{i}\right) \in C(X) \times X$, and there is no information about a preference order that might have generated the data: $A$ question then is whether or not there may be a consumer that has generated the data, i.e. is there a preorder $\succ, \sim$ such that $D \subset D_{d}(\succ)$.

Hence, problem of interest is to find restrictions that are imposed on consumer data by each of the given types of consumer behaviour $\mathcal{D}_{d}, \mathcal{D}_{z}$ and $\mathcal{D}_{c}$. For empirical work it is important to know whether these restrictions are strong or weak restrictions. We may also consider the assumption that such a preorder has certain properties. This again implies more restrictions, which can be studied.

To get an idea of the meaning of this questions, I will give some trivial examples of consumer behaviour. Later on I will investigate restrictions that the assumption of consumer demand imposes on consumer data.

Example 2.1 Obviously, one has $\mathcal{D}_{u}(f)=C(X) \times X$ for any constant function $f: X \rightarrow \mathbb{R}$. Similarly, one has $\mathcal{D}_{u}(\succsim)=C(X) \times X$ for the trivial preorder $\succ \sim$ defined on $X$ such that

$$
x \succsim y \quad(x, y \in X) \text {. }
$$

Example 2.2 One has $\mathcal{D}_{c}(\succsim)=C(X) \times X$ for the preorder $\succ, \sim$ defined on $X$ such that

$$
x \succsim y \Leftrightarrow x=y \quad(x, y \in X)
$$

So, with the above it is easy to find a cost minimizing or a utility maximizing preorder for a data set. For consumer demand, however, there are not such obvious solutions.

\subsection{Rationalizing Preference Orders and Utility Functions}

In the previous section $I$ described data sets that represent consumer behaviour for a given preference order or a utility function. This is the usual view on consumer behaviour in textbook theory. In empirical research, however, the point of view is another. Then one is ignorant of consumers preference order, or utility function, and one is curious how one may represent consumers preferences for available empirical data. This urges another theoretical basis, where the point of departure is an arbitrary data set of consumer behaviour $D \subset C(X) \times X$.

The existence of a utility function for consumer datia is related to an old problem which, as the Integrability Problem, has been traced as far back as Antonelli (1886). This is the problem to construct a utility function, which generates a given demand function. The given demand function $h(p, M)$ represents the demand of a consumer with income $M$ when the price vector $p$ is given. The 'Integrability Problem' now is the question whether there is a utility function $f$, such that the demand function can be derived from neoclassical consumer behaviour, li.e.

$$
f(h(p, M))=\sup _{p \times \leq \leq M} f(x) .
$$

The theory concerned with this problem can be divided into two main streams. In the early work of Antonelli a local analysis is made using differential equations. The last decades there has been another approach to the Integrability Problem*. In the pioneering work by Samuelson (1948, 1950), Houthakker (1950), and Uzawa (1960), the analysis is based on global characteristics with the axiom of revealed preference as a starting-point. For a review of results in revealed preference theory II refer to Chipman et 
al. (1971) and Richter (1979). The theory that shall bie presented in this book is also based on global characteristics. I will investigate whether given consumer data might be generated by a rational consumer using a preference order or a utility function. Therefore the following definition may be useful.

Definition: The family of preorders $>, \sim$ on $X$, which rationatize the data $D$, is defined as

$$
R_{d}(D)=\left\{\succeq \in X \times X \mid \succsim \text { is a preorder, } D \subset D_{d}(\beth)\right\}
$$

So $R_{d}(D)$ contains all the preorders that might have generated the data $D$. The family of utility functions $f: X \rightarrow \mathbb{R}$, which rationalize $D$, is analogously defined as

$$
\mathcal{F}_{d}(D) \equiv\left\{f: X \rightarrow \mathbb{R} \mid D \subset \mathcal{D}_{d}(f)\right\}
$$

The definitions of $\mathcal{R}_{u}, \mathcal{F}_{w}$, and $R_{c}, \mathcal{F}_{c}$ are analogous by replacing $\mathcal{D}_{d}$ by $\mathcal{D}_{u}$ and $\mathcal{D}_{\text {c }}$ respectively.

Clearly one has the following dual relation between the above defined families and sets of consumer data:

$$
\mathcal{F}_{d}\left(D_{1} \cup D_{2}\right)=\mathcal{F}_{d}\left(D_{1}\right) \cap \mathcal{F}_{d}\left(D_{2}\right)
$$

where $\mathcal{F}_{d}$ may be replaced by any of the other families $\mathcal{F}_{u ;} \mathcal{F}_{c}, R_{t}, R_{c}$ and $R_{d}$

\subsection{Data Enclosure}

In this section I will say something about the interesting idea of data enclosure, although it will not be used in this book. For the rationalizing set of utility functions $f: X \rightarrow \mathbb{R}$ we have

$$
\mathcal{F}_{d}(D)=\left\{f^{*}: X \rightarrow \mathbb{R} \mid f \in \mathcal{F}_{d}(d) \text { for all } d \in D\right\},
$$

using the abuse of motation $\mathcal{F}_{d}(d) \equiv \mathcal{F}_{d}(\{d\})$. Using this idea, we may generalize the definition of $D_{d}(f)$ in the following symmetrical way.

Definition: Let $F$ be a set of utility functions $f: X \rightarrow \mathbb{R}$ (or preorders). Then we define the following set of consumer demand data

$$
\mathcal{D}_{d}(F)=\left\{d \in C(X) \times X \mid d \in D_{d}(f) \text { for all } f \in F\right\} \text {. }
$$

Now, we may write $\mathcal{D}_{d}\left(\mathcal{F}_{d}(D)\right)$, where $D$ is a data set of consumer demand. This expression contains the data enclosure of $D$.

The data enclosure has the following meaning. If we observe data $D$ generated by consumer demand then, using this information and assuming the utility lunction $f$ unknown, the set $\mathcal{D}_{d}\left(\mathcal{F}_{d}(D)\right)$ is the maximal set of data that is known to be compatible with $D$.

If we add the hypothesis that the utility function $f$ satisfies $f \in F$, where $F$ is a family of utility functions, then the data enclosure is $\mathcal{D}_{d}\left(f \cap \mathcal{F}_{d}(D)\right)$. Now, the condition $f \in F$ means that we have more information available. Hence, the set $D_{d}\left(F \cap \mathcal{F}_{d}(D)\right)$ contains more data than $D_{d}\left(\mathcal{F}_{d}(D)\right)$. We have in general

$$
D \subset D_{d}\left(\mathcal{F}_{d}(D)\right) \subset D_{d}\left(F \cap \mathcal{F}_{d}(D)\right)
$$

\subsection{Efficiency Transformations}

This book describes several nomparametric tests of hypotheses that concern consumer and producer behaviour. The result of such tests has only two possibilities: the hypothesis is accepted or it is falsified. In empirical research it appears that the tested hypothesis is nearly always falsified by the nonparametric tests. We have to agree that falsification of the tested 
hypothesis does not mean that such a hypothesis is useless. In other cases we also accept errors: For example, when we try to estimate the parameters of a production function for given data. In that case we use a statistical measure to judge how serious these errors are. To develop such a measure for nonparametric tests, I introduce the idea of effictency tronsfomations. A feature of an efficiency transformation - important for empirical research is the possibility to relax the conditions belonging to effictent consumer behaviour. Efficiency transformations are a generalization of Afriat's idea of partial efficient consumer behaviour. Afriat (1973) introduced this idea to describe approximate utility maximization.

First, I will explain Afriat's idea. Consider the conditions for consumer demand, given a utility function $f: X \rightarrow R$ and price vector $p$. By the definition of consumer demand in the previous section, the hypothesis that $x \in X$ is consistent with consumer demand is equivalent to:

$$
\begin{array}{ll}
p x \leq p x^{\prime} \Rightarrow f(x) \leq f\left(x^{\prime}\right) & (x \in X) \\
p x<p x^{\prime} \Rightarrow f(x)<f\left(x^{*}\right) & (x \in X) .
\end{array}
$$

The idea of Afriat is to change this hypothesis into the weaker hypothesis

$$
\begin{array}{ll}
p x \leq e p x^{*} \Rightarrow f(x) \leq f\left(x^{*}\right) & (x \in X), \\
p x<e p x^{n} \Rightarrow f(x)<f\left(x^{n}\right) & (x \in X),
\end{array}
$$

where $e \in[0,1]$ is an efficiency measure. Then for $e=1$ we obtain the original hypothesis and by lowering $e$ this hypothesis is getting weaker.

I will generalize Afriat's idea of partial efficient behaviour by making use of efficiency transformations. The idea behind such a transtormation is simple. Efficiency transformations transform data into new data with the aim to relax the conditions for consumer demand. Such transformations $\phi_{e}$ are dependent on an efficiency parameter $e$ that indicates how serious we have deformed the data.

We may relax the conditions for consumer demand by changing the cost function $c$ to another cost function that increases the cost for all $x \neq x^{2}$. Then $a$ tighter budget restriction with less choice freedom will result in weaker conditions for consumer demand. The idea of Afriat can now be reformulated as follows.

Example 2.3: Consider the following transformation rule that transforms each cost function $c(x)$ into a function $c^{e}(x)$. This depends on a parameter $e$, called the efficiency level, and is such that

$$
c^{e}(x)= \begin{cases}c(x) & \left(x=x^{\prime}\right) \\ e^{-1} c(x) & \text { (otherwise) }\end{cases}
$$

This defines a transformation rule $\Phi_{e}$ that transforms every data element $\left(c, x^{\prime}\right)$ into $\left(c^{e}, x^{\prime}\right)$.

Now, let us consider the hypothesis $\left(c^{e}, x^{n}\right) \in \mathcal{D}_{d}(f)$, where $e \in[0,1]$, in the above example. In that case the conditions concerning $\left(c^{c}, x^{2}\right) \in \mathcal{D}_{d}(f)$ are equivalent to the conditions given by Afriat

$$
\begin{array}{ll}
c(x) \leq e c\left(x^{\prime}\right) \Rightarrow f(x) \leq f\left(x^{\prime}\right) & (x \in X), \\
c(x)<\operatorname{ec}\left(x^{\prime}\right) \Rightarrow f(x)<f\left(x^{\prime}\right) & (x \in X) .
\end{array}
$$

If $e<1$, clearly this hypothesis is weaker than the hypothesis that we have $\left(c, x^{\prime}\right) \in \mathcal{D}_{d}(f)$. The replacement of $c$ by $c^{e}$ allows the consumer to waste a fraction of $(1-e) c\left(x^{\prime}\right)$ of the expenditures. This is shown in Figure 2.1, where the consumer with cost function $c(x)=p x$ can achieve the same utillty as attained at $x^{\prime}$ by spending $e p x^{r}$ instead of $p x^{\prime}$. Note that in this figure one has $\left(c^{e}, x^{\prime \prime}\right) \in \mathcal{D}_{d}(f)$ for every $x^{\prime \prime}$ on $S$. 


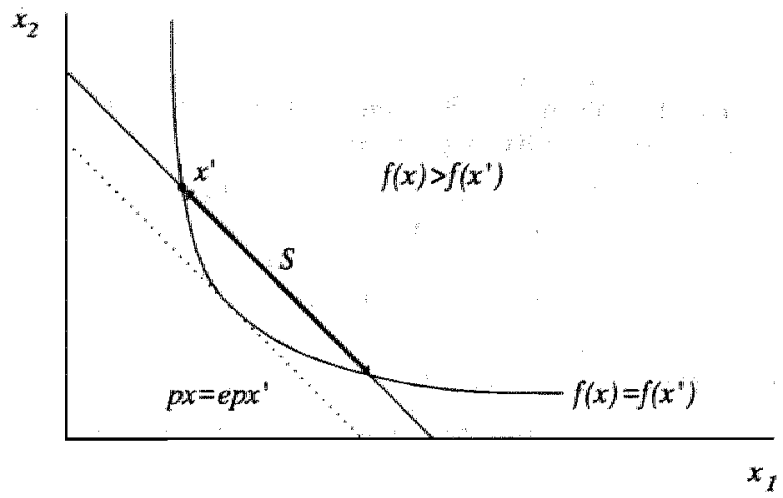

Figure 2.1 Partial Efficient Demand

So by using a transformation rule that changes all cost functions in a data set, we may weaken the hypothesis of consumer demand. For a general description of this idea, we need the following definition. It contains a summary of the properties of the given example.

Definition: An ef ficiency transformation $\Phi_{e}: C(X) \times X \rightarrow C(X) \times X$, where $e \in[0,1]$ is called the efficiency level, maps each data element $\left(c, x^{\prime}\right)$ on a data element $\Phi_{e}\left(c, x^{\prime}\right)=\left(c^{e}, x^{\prime}\right)$, such that $c^{e}\left(x^{\prime}\right)=c\left(x^{\prime}\right)$ and

$$
\begin{aligned}
& c^{a} \geq c^{b} \geq c^{1}=c \quad(0 \leq a \leq b \leq 1), \\
& c^{0}(x)=\infty \quad\left(x \in X: x \neq x^{\prime}\right) \text {. }
\end{aligned}
$$

For data sets $D$ we use the notation

$$
\Phi_{\mathrm{a}} D \equiv\left\{\Phi_{\mathrm{e}} d \mid d \in D\right\}
$$

Efficiency transformations are useful, because they provide an easy way to introduce an efficiency measure $e$. Using such an efficiency measure $e$ that depends on a data transformation, has the following advantage. Every: theory that can be applied to consumer data $D$ can also be applied to the transformed consumer data $\phi_{e} D$. Hence, theory concerning efficient belhaviour can directly be carried over to build a theory for inefficient behaviour.

The hypothesis that we have $\Phi_{e}\left(c_{\gamma} x^{\prime}\right) \in \mathcal{D}_{a}(f)$ is getting weaker when we lower the efficiency levell $e$. Thus clearly the following theorem is valid.

Theorem 2.1: Stppose $\Phi_{e}$ is an efficiency transformation and $a, e \in[0,1]$. Then

$$
\Phi_{e}\left(c, x^{2}\right) \in \mathcal{D}_{d}(\succsim) \Rightarrow \Phi_{a}\left(c, x^{*}\right) \in \mathcal{D}_{d}(\succsim) \quad(a \leq e) .
$$

This is also valid if $\mathcal{D}_{\mathrm{v}}$ is replaced by $\mathcal{D}_{u}$ or $\mathcal{D}_{c^{*}}$

In the applications of the theory, I will often use the simple efficiency transformation given in Example 2.3. 


\subsection{Efficiency Upper Bounds}

A feature of an efficiency transformation with an efficiency level $e$ is the possibility to relax the conditions belonging to efficient consumer behaviour. Suppose a given data set $D$ of consumer behawiour and we postulate that consumers preference order belongs to a certain family $F$ of preference orders. Then we are interested in the set of preference orders $F \cap R_{d}(D)$. If this set is empty then we may lower the efficiency level $e$ to find a nonempty set $F \cap R_{d}\left(\Phi_{e} D\right)$. By Theorem 2.1 the efficiency lewels $e$, for which one has $F \cap R_{d}\left(\Phi_{e} D\right) \neq \emptyset$, form always a set $[0, a]$ or $[0, a)$. We may use the upper bound $a$ to evaluate the hypothesis that consumers preference order belongs the family $F$ of preference orders. So the following definition is useful.

Definition: Let $D \subset C(X) \times X$ be a data set of consumer behaviour and let $F$ be a family of preorders on $X$. Then the efficiency upper bound $e_{d}(F, D)$ for efficiency transformation $\Phi_{e}$ is defined as

$$
e_{d d}(F, D) \equiv \sup \left\{e \in[0,1] \| F \cap R_{d}\left(\Phi_{e} D\right) \neq \varnothing\right\} .
$$

of course, this farmily $F$ of preference orders may be induced by a certain family of utility functions. Note that $e_{d}(F, D)$ is only well-defined when $F \cap \mathcal{R}_{d}\left(\Phi_{0} D\right) \neq \emptyset_{\text {, }}$ because otherwise $F \cap \mathcal{R}_{d}\left(\Phi_{e} D\right)=\emptyset$ for all $e \in[0,1]$. The following theorem shows when such a problem will not occur.

Theorem 2.2: Suppose $D$ is an arbitrary consumer data set and $\Phi_{e}$ an efficiency transfarmation. Then $R_{d i}\left(\Phi_{0} D\right)$ contains every reflexiwe prearder $\succ, \sim$

Proof: Let $\left(c, x^{*}\right) \in \Phi_{0} D$ and let $\succ_{2} \sim$ be a reflexive preorder. Then the definition of an efficiency transformation implies that we have $c(x)=\infty$ for all $x \neq x^{\prime \prime}$. So, we have

$$
c(x) \leq c\left(x^{\prime}\right) \Rightarrow x=x^{\prime} \Rightarrow x \precsim x^{\prime} \quad(x \in X),
$$

and hence $\left(c, x^{\prime}\right) \in \mathcal{D}_{u}(\succsim)$. Moreover, we have $\left(c, x^{\prime}\right) \in \mathcal{D}_{c}(\succsim)$, because $c(x) \geq c\left(x^{r}\right)$ for all $x \in X$.

The above theorem implies that $e_{d}(F, D)$ is well-defined if $F^{*}$ contains a preference order that is induced by a utility function, because such $a$ preorder is reflexive. Thus $e_{d}(F, D)$ is well defined for any nonempty family $F$ of utility functions.

Now, suppose we have a numerical test available that tests whether one has $F \cap R_{d d}\left(\Phi_{e} D\right) \neq \varnothing$ for arbitrary $e \in[0,1]$. In that case we can compute $e_{d d}(F, D)$ within any specified tolerance $\varepsilon>0$ by using the following simple bisection a.gorithm:

Step 1. Set $e_{0}=0, e_{1}=1$, and $e=1 / 2$.

Step 2. Repeat the following $n-1$ times, where $n$ is such that $2^{-n} \leq \varepsilon$ :

If $F \cap \mathcal{R}_{d}\left(\Phi_{e} D\right) \neq \emptyset_{3}$, put $e_{0}=e$, otherwise, put $\mathfrak{e}_{1}=e$;

Put $e=\left(e_{0}+e_{1}\right) / 2$.

Obviously the result will satisfy $\left|e_{d}(F, D)-e\right|<\varepsilon$.

\subsection{Existence of a Rationalizing Preference Order}

To define the axiom of revealed preference I will use the lollowing notation.

Definition: In case we consider consumer demand for the data $D=\left\{\left(c_{i}, x^{i}\right)\right\}_{i \in I}$, the binary relations $R(D)$ and $P(D)$ on $\left\{x^{i}\right\}_{i \in I}$ are defined as follows 


$$
\begin{array}{ll}
x^{i} R x^{j} \Leftrightarrow c_{i}\left(x^{j}\right) \leq c_{i}\left(x^{i}\right) & (i, j \in I) \\
x^{i} P x^{j} \Leftrightarrow c_{i}\left(x^{j}\right)<c_{i}\left(x^{i}\right) & (i, j \in I) .
\end{array}
$$

Table 2.1 Revealed Preferences

55555555556666666606067

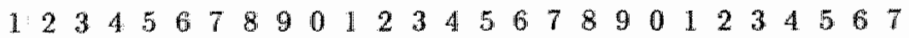

1951: $R P$

$1952 \cdot R$

$1953 P P R$

$1954 P P P R$

$1955 P P P P R$

1956 P P PPRPP

$1957 P P P P P \cdot R P$

$1958 P P P P P \cdot \cdot R$

1959PPPPPPPPR.

$1960 P P P P P P P P P R$

1961.PPPPPPPPPPR

$1962 P P P P P P P P P P P R$

1963 P P P PPPPPPPPR

$1964 P P P P P P P P P P P P P R$

1965 P P P P P P P P P P $R$

1966 P P P P P P P P P P PR

$1967 P P P P P P P P P P P P P P P P R$

1968 P P P P P P P P P P P P PR

$1969 P P P P P P P P P P P P P P P P P P R$

$1970 P P P P P P P P P P P P P P P P P P P R$

1971 $P P P P P P P P P P P P P P P P P P P P R$

$1972 P P P P P P P P P P P P P P P P P P P P P R$

$1973 P P P P P P P P P P P P P P P P P P P P P P R$

$1974 P P P P P P P P P P P P P P P P P P P P P P P R$

$1975 P P P P P P P P P P P P P P P P P P P P P P P P R$

1976 $P P P P P P P P P P P P P P P P P P P P P P P P P R$.

$1977 P P P P P P P P P P P P P P P P P P P P P P P P P P R$

So $x^{i} R x^{y}$ means that $x^{i}$ was at period $i$ available to the consumer. Further, $x^{i} P x^{j}$ means that there was even money lefti. These relations $R$ and $P$ have clearly the following meaning for consumer preferences:

$x^{i} R x^{j} \Rightarrow x^{j}$ is not preferred to $x^{i}$

$x^{i} P x^{j} \Rightarrow x^{i}$ is preferted to $x^{j}$.

Note that one has clearly $P \subset R$. As an example of such relations, the relations $P$ and $R$ of the Dutch consumption data are given in Table 2.1. The table shows that the later years are usually preferred above the earlier years, which is due to economical progress.

The axiom of revealed preference is based on the nearly trivial observation that data of consumer demand must satisfy: $x^{j}$ is not preferred to $x^{i}$ implies not; $x^{j}$ preferred to $x^{i}$. Assuming that consumers preferences are represented by a preorder, the meaning of $R$ holds too for the transitive closure $R^{*}$. So, consumer demand data must satisfy

$$
x^{i} R^{*} x^{j} \Rightarrow x^{j} \text { is not preferred to } x^{i} \Rightarrow \text { not } x^{j} P x^{i} \text {. }
$$

This is the axiom of revealed preference. 
Definition: $A$ set $D$ of consumer data is said to satisfy the axiom of revealed preference when

$$
x^{i} R(D)^{*} x^{i} \Rightarrow \operatorname{not} x^{i} P(D) x^{i} \quad(i, j \in I),
$$

which is equivalent to the statement $R(D)^{*} \cap P(D)^{c}=0$, (see Appendix A for the definition of the transitive closure $R^{*}$ of $R$, and the complement $P c$ of $P$.

In case cost functions are derived from price vectors, this axiom is reduced to the Generalized Axiom of Revealed Preference (GARP) defined in Varian (1982). The axiom of revealed preference is a necessary and sufficient condition for the existence of a rationalizing preorder. If the axiom is satisfied, we can even prove the existence of a rationalizing weak order. This result is contained in the following theorem that is even valid for data sets of an infinite number of elements.

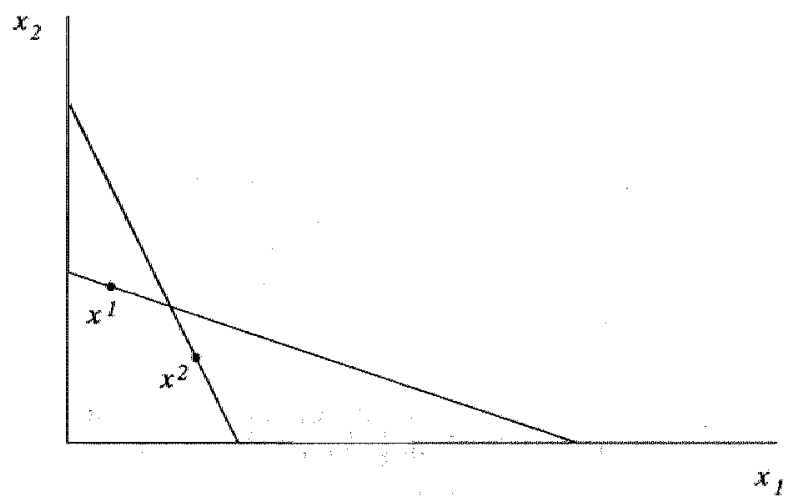

Figure 2.2 Inconsistent Behaviour

Theorem 2.3: Suppose $D=\left\{\left(c_{i}, x^{i}\right)\right\}_{i \in I}$ as set of consumet data. Them the following conditions are equivalent:

(i) $R_{d}(D) \neq \varnothing$.

(ii) There exists a weak order $>$, in $R_{d}(D)$.

(iii) $D$ satisfies the axion of revealed preference.

Proof: Define the binary relations $\bar{R}$ and $\bar{P}$ on $X$ by

$$
\begin{aligned}
& x \vec{R} y \Leftrightarrow \exists \vec{i} \in I: x^{i}=x \text { and } c_{i}(y) \leq c_{i}\left(x^{i}\right) \quad(x, y \in X) \text {, } \\
& x \vec{P} y \Leftrightarrow \exists i \in I: x^{*}=x \text { and } c_{i}(y)<c_{i}\left(x^{i}\right) \quad(x, y \in X) \text {. }
\end{aligned}
$$

According to the definition of $R_{d}(D)$, one has obviously

$$
R_{d}(D)=\left\{\searrow \subset X \times X \mid \succsim \text { is a preorder such that } \mathbb{R}<\_ \text {and } \vec{P} \subset>\right\}
$$

Now, from $\bar{P} \subset \bar{R}$ and Theorem $A .4$ in Appendix $A$, it follows that one has

$$
\text { (i) } \Leftrightarrow(\mathrm{ii}) \Leftrightarrow \bar{R}^{*} \cap \bar{P}^{c}=\emptyset \text {. }
$$

To prove the theorem it is thus sufficient to show the equality $\vec{R}^{*} \cap P^{c}=R^{*} \cap P c$. One can easily show that the following relations are vallid 


$$
\begin{array}{lc}
x^{j} \bar{R}^{*} x^{j} \Leftrightarrow x^{i} R^{*} x^{j} & (i, j \in I), \\
x^{i} \bar{P}_{x} \Leftrightarrow x^{i} P x^{j} & (i, j \in I) .
\end{array}
$$

This and the observation that we have

$$
\begin{aligned}
& x \vec{R}^{*} y \Rightarrow \exists i \in I: x=x^{i} \quad(x, y \in X), \\
& y \bar{P} x \Rightarrow \exists j \in I: y=x^{j} \quad(x, y \in X),
\end{aligned}
$$

imply the equivalence relation

$$
x \bar{R}^{*} y \text { and } y \bar{P} x \Leftrightarrow x R^{*} y \text { and } y P_{x} \quad(x, y \in X)
$$

we had to prove:

Figure 2.2 displays a simple example of price and quantity data, which does not satisfy the axiom of revealed preference. It has the following revealed preference table.

Table 2.2 Revealed Preferences

\section{2}

$1 R P$

$2 P R$

Summarizing the above in terms of symmetry and conserved properties, we have the following result. In generall we can obtain consumer data from a model of consumer behaviour that uses a preorder $\succ, \sim$. If we have data $d_{i}$, for different periods $i \in I$, then we might use in the general model for each period $i$ another preorder $>_{i}, \sim$. When we use the symmetry assumption that this preorder is time-independent, i.e.

$$
\succsim_{i}=\succsim_{j} \quad\left(i_{*}, j \in I\right),
$$

then we obtain the following conserved property. Sets of data $D \subset D^{\prime}$, both generated by the same time-independent preorder model, satisfy

$$
\succsim \in R_{d}\left(D^{\prime}\right) \Rightarrow R(D) \subset \succsim \text { and } P(D) \subset>\text {. }
$$

So the right hand restriction remains valid when new data is observed and added to $D$. It is a conserved property. This is in fact the basis of the axiom of revealed preference.

We can draw the following parallel for this result in classical mechanics. In classical mechanics a time-independent Lagrange function means that the energy will be conserved by the system. Ir economic science a timeindependent utility function implies the conservation of the revealed preferences. In both cases this result is independent from the specific form of the Lagrangian or utility function respectively.

\subsection{The Revealed Preference Axiom and Dutch Consumer Data}

Maks (1978, 1980), Landsburg (1981) and Varian (1982) tested aggregate price and quantity consumption data on the axiom of revealed preference. They find hardly any violations of axiom of revealed preference. The Dutch consumer data appear also to satisfy the axiom of revealed preference. In Table 2.3 the annual observations from Table 21 are ordered in such way that the resulting matrix is lower triangular. Such an ordering is only possible if the axiom of revealed preference is satisfied. To obtain this observation order from the natural year order, the observations for the years 1951 and 1952, and the years 1956 and 1958, are interchanged. As I will show in the following section, the resulting ordering of the years is compatible with 
increasing utility values for a rationalizing utility function.

At rirst sight it is striking that the lower triangle in Table 2.3 is completely filled, which is in general not necessary. This means that there is only one unique observation order that generates a lower triangle matrix. Furthermore, it means that the closure $R^{*}$ is equal to $R$, and we cannot increase - in this particular case - our information about consumer preferences by taking the closure over $R$.

Table 2.3 Revealed Preferences

$\begin{array}{lllllllllllllllllllllllllll}5 & 5 & 5 & 5 & 5 & 5 & 5 & 5 & 5 & 6 & 6 & 6 & 6 & 6 & 6 & 6 & 6 & 6 & 6 & 7 & 7 & 7 & 7 & 7 & 7 & 7 & 7\end{array}$

$\begin{array}{lllllllllllllllllllllllllll}2 & 1 & 3 & 4 & 5 & 8 & 7 & 6 & 9 & 0 & 1 & 2 & 3 & 4 & 5 & 6 & 7 & 8 & 9 & 0 & 1 & 2 & 3 & 4 & 5 & 6 & 7\end{array}$

1952

$1951 P R$

$1953 P P R$

1954 PPPR

$1955 P P P P R$

$1958 P P P P P R$

$1957 P P P P P P R$

1956 P P P P P P $R$

$1959 P P P P P P P P R$

$1960 P P P P P P P P P R$

$1961 P P P P P P P P P P R$

$1962 P P P P P P P P P P P R$

$1963 P P P P P P P P P P P P R$

$1964 P P P P P P P P P P P P P R$

$1965 P P P P P P P P P P P P P P R$

$1966 P P P P P P P P P P P P P P P R$

$1967 P P P P P P P P P P P P P P P P R$

$1968 P P P P P P P P P P P P P P P P P R$

$1969 P P P P P P P P P P P P P P P P P P R$

$1970 P P P P P P P P P P P P P P P P P P P R \ldots . . .$.

$1971 P P P P P P P P P P P P P P P P P P P P R \cdots \cdots$

$1972 P P P P P P P P P P P P P P P P P P P P P R \ldots .$.

$1973 P P P P P P P P P P P P P P P P P P P P P P R \cdots$

$1974 P P P P P P P P P P P P P P P P P P P P P P P R$

$1975 P P P P P P P P P P P P P P P P P P P P P P P P R$.

$1976 P P P P P P P P P P P P P P P P P P P P P P P P P R$.

$1977 P P P P P P P P P P P P P P P P P P P P P P P P P P R$

The cause of the filled lower trangle is a lack of variation in the price data, which was also reported by Varian (1982) for aggregate U.S. consumption data. Figure 2.3 illustrates why one may expect to obtain a filled lower triangle, when prices are slowly changing, while budgets are uncreasing. Then each budget restriction contains the previous quantity choices. The Dutch consumption data satisfy these properties, as is orten the case for aggregate consumption data, except for the innocent interchange of the observations of a few subsequent years.

\subsection{Existence of a Rationalizing Utility Function}

According to Theorem 2.3 the axiom of revealed preference is a necessary condition for the existence of a utility function, which rationalizes a giwen set of consumer data. In this section I prove a theorem which states that the axiom of revealed preference is also a sufficient condition, if the given 
data set has a finite number of elements. Thus, since the Dutch consumer data satisfy the axion of revealed preference, there exists a utility function for the Dutch consumer datat.

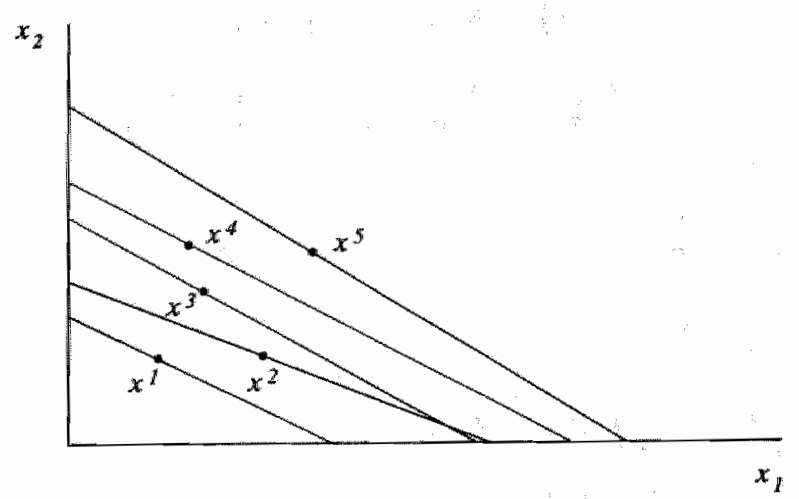

Figure 2.3 Little Price Variation

To prove the existence theorem a rationalizing utility function is constructed. For this construction we need the following theorem.

Theorem 2.4: Suppose $x^{i} \in X$ and $z_{i}: X \rightarrow \mathbb{R}$ for all $i \in I$, where $I$ is a finite index set. Let the binary relations $R$ and $P$ an $\left\{x^{i}\right\}_{\text {ier }}$ be defined by

$$
\begin{array}{ll}
x^{i} R x^{j} \Leftrightarrow z_{i}\left(x^{j}\right) \leq 0 & (i, j \in I), \\
x^{i} P x^{j} \Leftrightarrow z_{i}\left(x^{j}\right)<0 & (i, j \in I),
\end{array}
$$

and suppose there is a preorder $\succ$, on $\left\{x^{i}\right\}_{\text {iel }}$ such that $R \subset \succsim, P \subset \succ$. Then there exists a function.

$$
f(x)=\min _{i \in I} f_{i}+\lambda_{i} z_{i}(x) \quad(x \in X)
$$

such that $\lambda_{i}>0$ and $f\left(x^{i}\right) \geq f_{i}$ for all $i$. Moreover, if $>\sim$ is a weak order then the numbers $f_{i}$ can bo chosen in such a way that they represent this weak order.

Proor: If there is a preorder $\succ$ satisfying the above conditions, then by Theorem A.4 in Appendix A there is also a weak order that satisfies these conditions. 50 , we may assume that $r \sim$ is a weak order. Since $\succ, \sim$ is a weak order on a finite set, there is a finite sequence of elements

$$
x^{i}>x^{i} 2>\cdots>-x^{i} n_{1}
$$

such that $I$ is partitioned in equivalence classes

$$
\left.E_{l}=\left\{i \in I \| x^{i} \sim x_{i}\right\}\right\} \quad(l=1, \ldots, n),
$$

satisfying $E_{l} \cap E_{k}=\emptyset$ if $l \neq k$, and

$$
I=\bigcup_{l=1}^{n} E_{l} \text {. }
$$


Now, choose $f_{i}$ and $\lambda_{i}$ as follows. Put $f_{i}=\lambda_{i}=1$ for all $i \in E_{1}$, and for $l=2, \ldots$, fl do the following:

Step 1. Let

$$
\begin{aligned}
& \quad L_{i}<\min \left\{f_{j}+\lambda_{j} z_{j}\left(x^{i}\right), f_{j}\right\} \quad\left(i \in E_{3}, j \in \bigcup_{m \in l} E_{m}\right), \\
& \text { and put } f_{i}=L_{l} \text { for all } i \in E_{l}^{2} \text {. }
\end{aligned}
$$

Step 2. Then let

$$
U_{l} \geq\left(f_{j}-f_{i}\right) / z_{i}\left(x^{j}\right) \quad\left(i \in E_{l,} j \in \bigcup_{m<l} E_{m}^{\prime}\right)_{m}
$$

and put $\lambda_{i}=U_{l}$ for all $i \in E_{l}$.

Now, the condition $f_{i} \leq f\left(x^{i}\right)$ for all $i \in l$ follows clearly if we can show

$$
f_{i} \leq f_{j}+\lambda_{j} z_{j}\left(x^{i}\right) \quad(i, j \in I) .
$$

This inequality holds indeed, because our choice of $f_{i}$ and $\lambda_{i}$ in conjunction with the assumptions for the weak order $\succ, \sim$, imply

$$
\begin{aligned}
& x^{j} \sim x^{i} \Rightarrow f_{j}=f_{i} \text { and } z_{j}\left(x^{i}\right) \geq 0 \quad(i, j \in I), \\
& x^{j} \succ x^{i} \Rightarrow f_{i}<f_{j}+\lambda_{j} z_{j}\left(x^{i}\right) \quad(i, j \in I), \\
& x^{j}<x^{i} \Rightarrow \lambda_{j} \geq\left(f_{i}-f_{j}\right) / z_{j}\left(x^{i}\right) \text { and } z_{j}\left(x^{i}\right)>0 \quad(i, j \in I) .
\end{aligned}
$$

Finally, the numbers $f_{i}$ are clearly chosen in such a way that they represent. the weak order $\succ_{\text {, } ~}$.

Now we are able to prowe the main theorem which states necessary and sufficient conditions for the existence of a rationalizing utility function. In Afriat (1967) such a theorem was proved, which is improved by Varian (1982, 1983). I will generalize Varian's results by using a more general definition of consumer demand. The results improve a theorem given by Afriat (1973), concerning inefficient consumer demand for given prices, and generalize it by using cost functions.

Theorem 2.5: Suppose $D=\left\{\left(c_{i,} x^{i}\right)\right\}_{i \in I}$ is a finite data set of consumer behaviour.

(a) Then the following conditions are equivalent:

(i) $\mathcal{F}_{d}(D) \neq \emptyset$.

(ii) $D$ satisfies the axion of revealed preference.

(iii) There exist numbers $\lambda_{i}>0$ and $f_{i}$ such that

$$
f_{i} \leq f_{j}+\lambda_{j}\left[c_{j}\left(x^{i}\right)-c_{j}\left(x^{j}\right)\right] \quad(i, j \in I) .
$$

(b) One has $f \in \mathcal{F}_{d}(D)$ for every function of the form

$$
f(x)=\min _{i \in \mathbb{I}} f_{i}+\lambda_{i}\left[c_{i}(x)-c_{i}\left(x^{i}\right)\right] \quad(x \in X)
$$

where $f_{i}$ and $\lambda_{i}>0$ satisfy the conditions in (iii).

Proof (a) (i) $\Rightarrow$ (ii): If $\mathcal{F}_{d}(D) \neq \emptyset$ then $\mathcal{R}_{d}(D) \neq \emptyset$, hence (ii) follows rrom Theorem 2.3.

(ii) $\Rightarrow$ (iii): Put

$$
z_{i}(x)=c_{i}(x)-c_{i}\left(x^{i}\right) \quad(x \in X, i \in I) .
$$

From theorem 2.3 it follows that, if the axiom of revealed preference is satisfied, there is a rationalizing weak order. Hence, we may apply Theorem 2.4 to obtain a function

$$
f(x)=\min _{i \in I} f_{i}+\lambda_{i} z_{i}(x) \quad(x \in X)
$$

where $f_{i}$ and $\lambda_{i}$ satisfy the conditions in (iii).

(iii) $\Rightarrow$ (i): From (iii) it follows that the function $f$, defined ass above in 
the proof of $(i i) \Rightarrow(11)$, satisties

$$
\begin{array}{ll}
z_{i}(x) \leq 0 \Rightarrow f(x) \leq f_{i}=f\left(x^{i}\right) & (i \in I, x \in X) \\
z_{i}(x)<0 \Rightarrow f(x)<f_{i}=f\left(x^{i}\right) & (i \in I, x \in X) .
\end{array}
$$

Hence $f \in \mathcal{F}_{d}(D)$.

(b) That we thave $f \in \mathcal{F}_{d}(D)$ follows from the proof of $(i i) \Rightarrow(1)$.

The above theorem can be extended to a theorem in which the utility functions are restricted to a class of functions with one or more of the following properties: concave, strictly concave, monotonically increasing, strictly monotonically increasing, continuous, uniformly continuous. The proof of such a theorem is easy, if we suppose all cost functions $c_{i}$ are element of this class of functions. In that case the constructed utility function $f$ in Theorem 2.5 (b) will be an element of this class too. So the mentioned properties of the cost functions can be carried over to properties of the utility function.

Now, the following problem occurs if we try to prove the exisitence of a continuous function for inefficient behaviour. We may get into problems if we use an efficiency transformation $\Phi_{e}$, because this may introduce a discontinuity in the cost functions. The transformation $\Phi_{1}\left(c, x^{\prime}\right)$ increases all walues of the cost function $c$, except the value $c\left(x^{\prime \prime}\right)$ at $x^{\prime}$. So in general we are left with a discontinuily at $x^{\prime}$ after the transformation of a continuous cost function. This means that, for data of the form $\Phi_{e} D_{\text {, }}$ discontinuities will be carried over to the constructed utility function. Such discontinuities have the following property.

Theorem 2.6: Suppose $c: X_{\rightarrow} \rightarrow \mathbb{R}_{+}$is contintwous at $x^{\prime}$, and $\Phi_{e}$ is an efficiency transformation. Then $\Phi_{e}\left(c, x^{\prime}\right)=\left(c^{e}, x^{\prime}\right)$ satisfies

$$
\lim _{x \rightarrow x^{\prime}} c^{e}(x) \geq c^{e}\left(x^{\prime}\right)
$$

Proof: For an efficiency transformation one has in general $c^{e}\left(x^{\prime}\right)=c\left(x^{\prime}\right)$ and $c^{g}(x) \geq c(x)$ for all $x \in X$. Hence, we have

$$
\lim _{x \rightarrow x^{\circ}} c^{e}(x) \geq \lim _{x \rightarrow x^{\circ}} c(x)=c\left(x^{*}\right)=c^{e}\left(x^{\prime}\right)
$$

where we have used the continuity of $c$ at $x^{2}$.

The following theorem shows how such discontinuities may be fixed.

Theorem 2.7: Suppose $D=\left\{\left(c_{i}, x^{i}\right)\right\}_{\text {ieI }}$ is a finite data set of consumer behaviour and $f_{i}$ and $\lambda_{i}>0$ satisfy

$$
f_{i} \leq f_{j}+\lambda_{j}\left[c_{j}\left(x^{i}\right)-c_{j}\left(x^{j}\right)\right] \quad\left(i_{i}, j \in I\right) .
$$

Then whe has $f \in \mathcal{F}_{d}(D)$ for every function of the form

$$
f(x)=\min _{i} f_{i}+\lambda_{i}\left[\hat{c}_{i}(x)-c_{i}\left(x^{i}\right)\right] \quad(x \in X),
$$

where all the finctions $\hat{c}_{i}$ satisfy

$$
\begin{array}{ll}
\hat{c}_{i}(x) \geq c_{i}(x) & \left(x=x^{i}\right)_{,} \\
\hat{c}_{i}(x)=c_{i}(x) & \text { (otherwise). }
\end{array}
$$

Proof: The construction of $f$ implies that we have $f\left(x^{i}\right) \geq f_{i}$ for all $i \in I$. Now, let $i \in I$ and suppose $x \neq x^{i}$. Then we have $c_{i}(x)=\hat{c}_{i}(x)$ and thus

$$
c_{i}(x) \leq c_{i}\left(x^{i}\right) \Rightarrow f(x) \leq f_{i} \leq f\left(x^{i}\right), c_{i}(x)<c_{i}\left(x^{i}\right) \Rightarrow f(x)<f_{i} \leq f\left(x^{i}\right)
$$

Furthermore, in case $x=x^{i}$, we have

$$
c_{i}(x) \leq c_{i}\left(x^{i}\right) \Rightarrow f(x)=f\left(x^{i}\right) .
$$


Hence $f \in F_{d}(D)$.

From the above theorems we can derve the following theorem in which we remove the discontinuity, caused by an efficiency transformation.

Theorem 2.8: Suppose $\left\{\left(c_{i} x^{i}\right)\right\}_{\text {ieI }}=\Phi_{e} D$, where $D$ is a finte data set and $\phi_{e}$ is an efficiency transformation. Put

$$
\hat{c}_{i}(x)=\lim _{x \rightarrow \infty} c_{i}(v) \quad(i \in I, x \in X)
$$

Suppose all functions $\hat{c}_{i}$ and the cost functions of $D^{\prime}$ are continnaws, and the cost functions $c_{i}$ have only one discontinuity at $x^{i}$. Then $\mathcal{F}_{d}\left(\Phi_{0} D\right)$ the existence of a continuous function $f \in \mathcal{F}_{d}\left(\Phi_{e} D\right)$

Proof: Suppose $\mathcal{F}_{d}\left(\Phi_{e} D\right) \neq 0$. Then by Theorem 2.5 there are numbers $\lambda_{i}>0$ and $f_{i s}$ such that $f_{i} \leq f_{j}+\lambda_{j}\left[c_{j}\left(x^{i}\right)-c_{j}\left(x^{j}\right)\right]$ for all $i, j \in I$. The functions $\hat{c}_{i}$ are continuous functions, equal to $c_{i}$ except at $x^{i}$, where the discontinutity is removed. By Theorem 2.6 such functions satisfy $\hat{c}_{i}\left(x^{i}\right) \geq c_{i}\left(x^{i}\right)$ for all $i \in I$. Hence by Theorem 2.7 , we have $f \in \mathcal{F}_{d}(D)$ for the function

$$
f(x)=\min _{i \in I} f_{i}+\lambda_{i}\left[\hat{c}_{i}(x)-c_{i}\left(x^{i}\right)\right] \quad(x \in \mathbb{X}) .
$$

This function is continuous, because all functions $\hat{c}_{i}$ are continuous.

If we apply the efficiency transformation of Example 2.3 to price and quantity data; we may use the above theorem to prove the existence of a concave continuous utility function.

Theorem 2.9: Suppose $D$ is a finite price and quantity date set and $\phi_{\mathrm{e}}$ is the efficiency transformation of Example 2.3. Then $\mathcal{F}_{d}\left(\Phi_{e} D\right) \neq \emptyset$ implies the existence of a concave continuods function $f \in \mathcal{F}_{d}\left(\Phi_{e} D\right)$.

Proof: From the proof of Theoren 2.8 it follows that properties of the cost functions $\hat{c}_{i}$, where the discontinuity is removed, can be carried over to the constructed utility function $f$. If we apply the efficiency transformation of Example 2.3 to price and quantity data then we will obtain concave continuous functions $\hat{c}_{i}$ in Theorem 2.8. Hence, in the proof of Theorem 2.8, we obtain the existence of a concave continuous function $f \in \mathcal{F}_{d}\left(\Phi_{e} D\right)$, assuming that we have $\mathcal{F}_{d}\left(\Phi_{e} D\right) \neq 0$. 


\section{LINEARLY HOMOGENEOUS CONSUMER DEMAND}

\subsection{Introduction}

The question whether there exists a linearly homogeneous utility function for a given data set, which is compatible with efficient neoclassical consumer behaviour, is treated in Afriat (1972, 1981), Diewert (1973), and Varian (1983). In the present chapter I generalize their results, using the definition of consumer demand, given in Chapter 2 , and formulate a necessary and surficient condition which has to be satisfied by linearly homogeneous consumer data. In contrast to earlier results, concerning the existence of am arbitrary rationalizing utility function, this condition can be applied to both finite and infinite data sets. The results can allso be applied to the more general case where utility functions are assumed to be homothetic, because a homothetic function is a strictly increasing transformation of a linearly homogencous function.

Another point of interest is the existence of lower and mpper bounds for the linearly homogeneous utility function for given consumer denand data. These bounds follow from the symmetry of a consumer demand model in case utility and cost functions are linearly homogeneous. Such bounds may give an impression of consumers utility function without the need of a parametric specification of the utility function and estimation of the parameters.

\subsection{Linearly Homogeneous Utility Functions}

I consider in this chapter linearly homogeneous functions $f: X \rightarrow \mathbb{R}$ where the choice space $X$ is cone.

Definition: A cone is a set $X$ such that

$$
\lambda x \in X \quad(x \in X, \lambda \geq 0) \text {. }
$$

Definition: A function $f: X \rightarrow \mathbb{R}$ is said to be linearly homogeneows when

$$
f(\lambda x)=\lambda f(x) \quad(x \in X, \lambda \in \mathbb{R}) \text {. }
$$

An alternative way to define linear homogeneity is the following, where the idea of symmetry is used. A linearly homogeneous function is a function that is invariant under the family of transformations $\boldsymbol{T}_{\lambda}$ defined as

$$
\left(T_{\lambda} f\right)(x)=\lambda^{-1} f(\lambda x) \quad\left(\lambda \neq 0, x \in X^{\prime}\right)
$$

Remark: Note that $f(0)=0$ follows in the first definition from $f(0)=\lambda f(0)$ with $\lambda=0$ and in the secord definition from $\lambda^{-1} f(0)=f(0)$ for every $\lambda \neq 0$.

The underlying idea of the theory in this chapter is the following. Consider the optimization problem

$$
\sup _{x \rightarrow \leq c(x)} f(x) \text {. }
$$

Using a Lagrangian

$$
L\left(x, x^{\prime}\right)=f(x)-u\left[c(x)-c\left(x^{\prime \prime}\right)\right] \quad(x \in X)
$$

this problem can be transformed into the unconstrained optimization problem

$$
\sup _{x} L\left(x, x^{\prime}\right)
$$


where the Lagrangian is maximized. When both $f$ and $c$ are linearly homogeneous then this Lagrangian has a symmetric property, because one has

$$
L\left(\lambda x, \lambda x^{n}\right)=\Lambda\left(x, x^{n}\right) \quad\left(\lambda \in \mathbb{R}, x, x^{\prime} \in X\right) .
$$

The existence of such a symmetry implies that we can derive a conservation law for linearly homogeneous consumer demand.

The following theorem states that linearly homogeneous consumer demand consists of rays of consumer data.

Theorem 3.1: Suppose $f: X \rightarrow \mathbb{R}$ is limedrly homogencous and $X$ is a cone. Then

$$
\left(c, x^{\prime \prime}\right) \in D_{d}(f) \Leftrightarrow\left(T_{\lambda} c_{7} \lambda x^{\prime}\right) \in D_{d}(f) \quad(\lambda>0)
$$

where the transformation $\boldsymbol{T}_{\lambda}$ is defimed by

$$
\left(\boldsymbol{T}_{\lambda} c\right)(x)=\lambda^{-1} c(\lambda x) \quad(x \in X)
$$

Proof: The elementary proof is left to the reader.

So, by the above theorem the symmetry $\boldsymbol{T}_{\lambda} f=f$, that holds for linearly homogeneous functions, results in the symmetry $\left(c, x^{\prime}\right) \sim\left(T_{\lambda} c, \lambda x^{\prime}\right)$ of the corresponding consumer demand data, where we may write $T_{\lambda} c=c$ in case $c$ is linearly homogeneous.

Figure 3.1 displays an example of consumer demand for a linearly homogeneous function.

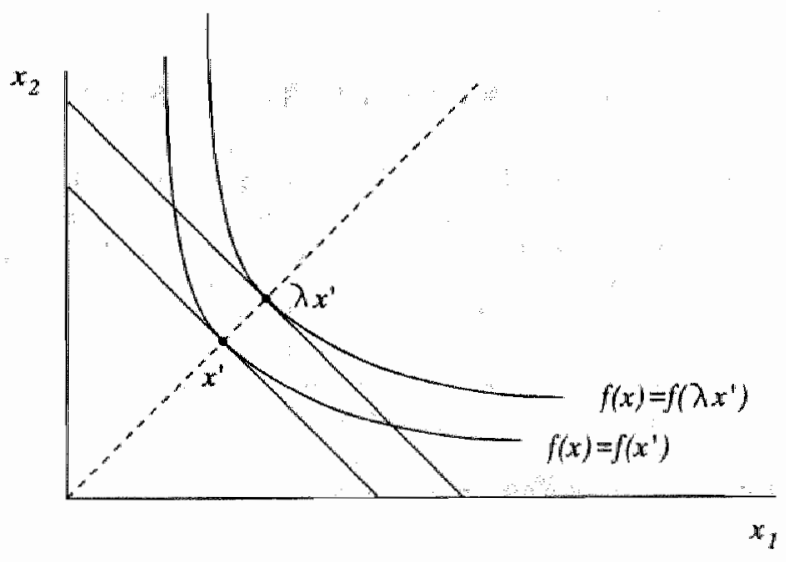

Figure 3.1. Linearly Homogeneous Function

Below 1 prove that linearly homogeneous utility maximization is equivalent to linearly homogeneous cost minimization, assuming that the attained utility value is positive.

Theorem 3.2: Suppose $f: X \rightarrow \mathbb{R}$ and $c: X \rightarrow \mathbb{R}_{+}$are linearly homogeneows, $X$ is a cone, $x^{\prime \prime} \in X, c\left(x^{\prime}\right)>0$ and $f\left(x^{\prime}\right)>0$. Then $\left(c, x^{\prime}\right) \in \mathcal{D}_{n}(f)$ if and only if $\left(c, x^{\prime \prime}\right) \in \mathcal{D}_{0}(f)$.

Proof $(\Rightarrow)$; Let $\left(c, x^{*}\right) \in D_{u}(f)$ and $x \in X$. Suppose $c(x)<c\left(x^{\prime}\right)$. Then one has $f(x) \leq f\left(x^{\prime}\right)$, since $\left(c, x^{\prime \prime}\right) \in \mathcal{D}_{u}(f)$, and we may take $\lambda>1$ such that $\lambda c(x)<c\left(x^{\prime}\right)$. Now, assume that we have $f(x)=f(x)$. Then 


$$
c(\lambda x)=\lambda c(x)<c(x), f(\lambda x)=\lambda f(x)>f(x), \lambda x \in X
$$

This contradicts however $\left(c, x^{\prime}\right) \in D_{u}(f)$. Thus $f(x) \neq f\left(x^{\prime}\right)$, which implies that we have $f(x)<f\left(x^{\prime}\right)$. This means $\left(c_{*} x^{\prime}\right) \in D_{d}(f)$ according to the definition of $\mathcal{D}_{c}$.

$(-1)$ Let $\left(c, x^{\prime}\right) \in D_{c}(f)$ and $x \in X$. Suppose $f(x)>f\left(x^{4}\right)$. Then $c(x) \geq c\left(x^{*}\right)$, since $(c, x) \in D_{c}(f)$, and we may take $\lambda<\mathbb{1}$ such that $f(x)>f\left(x^{2}\right)$. Now, assume $c(x)=c\left(x^{2}\right)$. Then

$$
c(\lambda x)=\lambda c(x)<c(x), f(\lambda x)=\lambda f(x)>f(x), \lambda x \in X .
$$

However, this contradicts $\left(c, x^{\prime}\right) \in \mathcal{D}_{c}(f)$ and we have thus $c(x) \neq c\left(x^{*}\right)$. This means $c(x)>c\left(x^{\prime}\right)$, and hence $\left(c, x^{\prime}\right) \in D_{n s}(f)$.

The following theorem show a property of data that is generated by a consumer using a linearly homogeneous utility function.

Theorem 3.3: Suppose $f: X \rightarrow \mathbb{R}$ is linearly homogeneous and $X$ is a cone. Then

$$
(c, x) \in \mathcal{D}_{d}(f) \Rightarrow c\left(f\left(x^{\prime}\right) f(x)^{-1} x\right) \geq c\left(x^{\prime}\right) \quad(x \in X: f(x)>0) \text {. }
$$

Proof: Since $f$ is linearly homogeneous, one has $f\left(f\left(x^{\prime}\right) f(x)^{-1} x\right)=f\left(x^{4}\right)$. Thus $\left(c, x^{\prime}\right) \in D_{c}(f)$ implies $c\left(f\left(x^{\prime}\right) f(x)^{-1} x\right) \geq c\left(x^{\prime}\right)$.

The following theorem prowides a condition which guarantees that $f\left(x^{n}\right)>0$ is satisfied.

Theorem 3.4: Suppose $f: X \rightarrow \mathbb{R}$ and $c: X \rightarrow \mathbb{R}_{+}$are linearly homogeneons functions, $X$ is a cone, and $c\left(x^{\prime}\right)>0$. If $\left(c, x^{\prime}\right) \in \mathcal{D}_{c}$ (f) then $f\left(x^{\prime}\right)>0$.

Proof: Since $X$ is a cone it contains the origin. Furthermore, since $f$ and $c$ are linearly homogeneous, one has $f(\mathbf{0})=c(0)=0$. Now, $c(\mathbf{0})=0<c\left(x^{\prime}\right)$ and $\left(c, x^{\prime \prime}\right) \in \mathcal{D}_{d}(f)$ implies $f(0)=0<f\left(x^{\prime \prime}\right)$.

\subsection{Existence of a Rationalizing Linearly Homogeneous Function}

Using the above theorems, I am able to prove a necessary and sufficient condition for the existence of a linearly homogeneous utility function, which rationalizes consumer data. The condition refers to the idea of an absorptive matrix. Such a matrix can be defined as follows. Let $A=\left[a_{i j}\right], i, j \in I$, be a matrix for which $a_{i j}$ represents the cost of moving from $i$ to $j$. Then $A$ is called an absorptive matrix when the total cost of every cycle path is nonnegative. For instance, for the cycle path $i \rightarrow j \rightarrow k \rightarrow i$ this means $a_{i j}+a_{j k}+a_{k i} \geq 0$. More information concerning absorptive matrices is given in Appendix $C$. This appendix describes, for instance, a simple algorithm which tests whether a matrix is absorptive. At this point we shall confine ourselves to give the following definition.

Definition: $A$ matrix $A \in \mathbb{R}^{n} \times \mathbb{R}^{n}$ is said to be absorptive when

$$
a_{i j}+a_{j k}+\cdots+a_{l m}+a_{m i i} \geq 0
$$

for every possible sequence $i, j, k, \ldots, t, m \in l$.

This definition is used in the following theorem that considers necessary and sufficient conditions for the existence of a linearly homogeneous utility
function.

Theorem 3.5: Suppose $D=\left\{\left(c_{i}, x^{i}\right)\right\}_{\text {iel }}$ is a data set of consumer behaviour, where $c_{i}: X \rightarrow \mathbb{R}_{+}$are linearly homogeneous and $c_{i}\left(x^{i}\right)>0$, for all $i \in I$, and $X$ is $a$

(a) Then the following conditions are equivalent:

(i) There exists a linearly homogeneows function $f \in \mathcal{F}_{a}(D)$.

(ii) There exists a linearly homogeneous function $f \in \mathcal{F}_{a}(D)$.

(iii) There exist numbers $\lambda_{i}>0$ such that 


$$
\lambda_{i} \lambda_{j}^{-1} c_{i}\left(x^{j}\right) \geq c_{i}\left(x^{i}\right) \quad(i, j \in I)
$$

(iv) The matrix $A$ is absorptive, where

$$
a_{i j}=\ln \left(c_{j}\left(x^{i}\right) / c_{j}\left(x^{j}\right)\right) \quad(i, j \in I) .
$$

(b) If the above conditions are satisfied then one has $f \in \mathcal{F}_{\text {al }}(D)$ for every function of the form

$$
f(x)=\inf _{i \in \mathbb{I}} \lambda_{i} c_{i}(x) / c_{i}\left(x^{i}\right) \quad(x \in X),
$$

such that the numbers $\lambda_{i}$ satisfy the condition given in (iii).

Proof (a) (i) $\Rightarrow$ (ii): Trivial.

(ii) $\Rightarrow$ (iii): Suppose $f \in \mathcal{F}_{c}(D)$ is a linearly homogeneous function. Then by Theorem 3.4 one has $f\left(x^{i}\right)>0$ for all $i \in l$. Thus from Theorem 3.3 it follows that

$$
c_{i}\left(f\left(x^{i}\right) f\left(x^{j}\right)^{-1} x^{j}\right) \geq c_{i}\left(x^{i}\right) \quad(i, j \in I) .
$$

Hence the numbers $\lambda_{i}=f\left(x^{i}\right)$ satisfy condition (iii) , since the functions $c_{i}$ are by assumption linearly homogeneous.

(iii) $\Rightarrow$ (i) : Put

$$
f(x)=\inf _{i \in I} \lambda_{i} c_{i}(x) / c_{i}\left(x^{i}\right) \quad(x \in X) .
$$

Since $\lambda_{i} \lambda_{j}^{-1} c_{i}\left(x^{j}\right) \geq c_{i}\left(x^{i}\right)$ for all $i, j \in l$, one has now

$$
f\left(x^{j}\right)=\inf _{i \in I} \lambda_{i} c_{i}\left(x^{j}\right) / c_{i}\left(x^{i}\right) \geq \lambda_{j} \quad(j \in I) \text {. }
$$

To show that $f \in \mathcal{F}_{d}(D)$, let $x \in X$ and $i \in I$. Suppose $c_{i}(x) \leq c_{i}\left(x^{i}\right)$. Then clearly $f(x) \leq \lambda_{i} \leq f\left(x^{i}\right)$. Similarly, $f(x)<\lambda_{i} \leq f\left(x^{i}\right)$ if $c_{i}(x)<c_{i}\left(x^{i}\right)$, thus $f \in \mathcal{F}_{d}(D)$. Further, since all functions $c_{i}$ are by assumption element of $F$, one has obviously $f \in F$.

(iii) $\Leftrightarrow$ (iv): Using $\phi_{i}=\ln \lambda_{i}$, one has (iii) equivalent to the existience of numbers $\phi_{i}$ such that

$$
\phi_{i}-\phi_{j} \leq a_{i j} \quad(i, j \in I) \text {. }
$$

Theorem C.2, given in Appendix C, sthows that this is equivalent to the condition that $A$ is absorptive.

(b) That we have $f \in \mathcal{F}_{d}$ (D) follows from the proof of (iii) $\Rightarrow$ (i).

Figure 3.2 displays an example of price and quantity data for efricient linearly homogeneous consumer behaviour. The quantity data $x^{\text {is }}$ is multiplled by $\lambda_{i}$, where $\lambda_{i}$ satisfles condition (iii) in Theorem 3.5 . We may interpret these numbers as $\lambda_{i}=f\left(x^{*}\right)$ of a rationalizing linearly homogeneous function $f$. The figure shows how the numbers $\lambda_{i}$ are rellated to the corresponding unit isoquant.

Theorem 3.5 can be extended to a theorem where the utility functions are restricted to a class of functions with one or more of the following properties. concave, monotonically increasing, upper semicontinuous. Furthermore, when the data set is finite, we may include the properties: strictly concave, strictly monotonically increasing, continuous, uniformily continuous. For a simple proof of such a theorem, we need the assumption that all cost functions $c_{i}$ has such a property. Then the consucucted utility function $f$ in the above proof has this property too. 


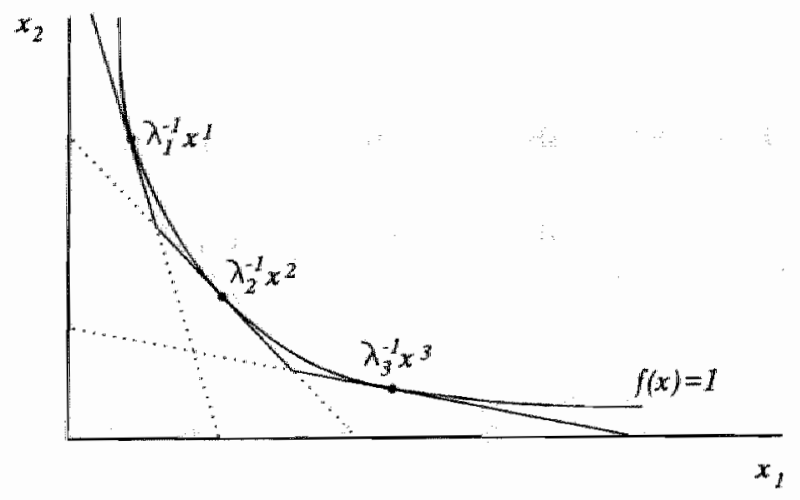

Figure 3.2 Linearly Homogeneous Behaviour

We can translate the above results for linearly homogeneous consumer demand in tems of symmetry and conserved properties as follows. The symmetry assumption is that the utility function and cost functions are invariant under the family of transformations $\boldsymbol{T}_{\lambda}$ such that

$$
\left(T_{\lambda} f\right)(x)=\lambda^{-1} f(\lambda x) \quad(\lambda \neq 0) \text {. }
$$

The consumer demand conditions for which this symmetry holds are invariant under translations in the commodity space along the rays with the origin as source. So, for the data we have the symmetry

$$
\left(c, x^{\prime}\right) \in \mathcal{D}_{d}(f) \Leftrightarrow\left(c, \lambda x^{\prime}\right) \in \mathcal{D}_{d d}(f) \quad(\lambda>0) \text {. }
$$

This implies that sets of data $D$ generated by the linearly homogeneous model, satisfy

$$
f \in \mathcal{F}_{d}(D) \text { linearly homogeneous } \Rightarrow f(x) \leq f\left(x^{\prime}\right) e(x) / c\left(x^{\prime}\right) \text { for }\left(c, x^{\prime}\right) \in D,
$$

assuming that we have $c\left(x^{\prime}\right)>0$. This property is the basis of Theorem 3.5. The right hand inequalities remain valid when we add more data, generated by the same model, to $D$. So me may consider it as a conserved property. We can draw a parallel for this result in classical mechanics, where a Lagrange function that is translation invariant in space implies the conservation of momentum.

\subsection{The Existence Problem in Case of Inefficiency}

If we wish to apply theorem 3.5 to transformed data $\Phi_{e} D$, where $\Phi_{e}$ is an efficiency transformation, then the transformed cost functions have to be linearly homogeneous. So we may get into problems when using the efficiency transformation of Example 2.3 in which $\Phi_{e}\left(c, x^{\prime}\right)=\left(c^{e}, x^{\prime}\right)$ is defined by

$$
c(x)= \begin{cases}c(x) & \left(x=x^{\prime}\right), \\ e^{-1} c(x) & \text { (otherwise) }\end{cases}
$$

The result $c^{e}$ is in that case in general not, a linearly homogeneous function, 
assuming that $c$ has this property. A slightly different efficiency transformation, that does transform Hinearly homogeneous cost functions into linearly homogeneous cost functions, is the following.

Example 3.1: Let the efficiency transformation $h_{e}\left(c, x^{c}\right)=\left(c^{e}, x^{*}\right)$ be such that

$$
c^{e}(x)= \begin{cases}c(x) & \left(x=\lambda x^{*}, \lambda \in \mathbb{R}\right), \\ e^{-1} c(x) & \text { (otherwise) }\end{cases}
$$

Still there remains to solve the same problem as in the previous chapter. What to do when we are searching for a continuous utility function and the efficiency transformation destroys the continuity of the cost functions? In that case we shall not derive a continuous utility function in the proof of Theorem 3.5. To solve this problem we may try to fix the discontinuities in the same way as in Theorem 2.8 in the previous chapter. This approach results in the following theorem.

Theorem 3.6: Suppose $\Phi_{e} D=\left\{\left(c_{i}, x^{i}\right)\right\}_{i \in I}$ is a finite data set, where $\Phi_{e}$ is an efficiency transformation, $c_{i}: X \rightarrow \mathbb{R}_{+}$are linearly homogeneous and $c_{i}\left(x^{i}\right)>0$, for all $i \in I_{3}$ and $X$ is a cone. Suppose every function $c_{\hat{i}}$ is only discontinuous at $\left\{\lambda x^{i} \mid \lambda \in \mathbb{R}\right\}$ and put

$$
\hat{c}_{i}(x)=\lim _{v \rightarrow x} c_{i j}(v) \quad(i \in I, x \in X)
$$

where $v \notin\left\{\lambda x^{i} \mid \lambda \in \mathbb{R}\right\}$ when we take the limit $v \rightarrow x$. Suppose all functions $\hat{c}_{i}$ and the cost functions of $D$ are continuous. If there is a linearly homogeneous function in $\mathcal{F}_{d}\left(\Phi_{e} D\right)$ then there exists a continuous inearly homogeneous function $f \in \mathcal{T}_{d}\left(\Phi_{e} D\right)$.

Proof: The proof is basically the same as the proof of Theorem 2.8. By replacing $c_{i}$ by $\hat{c}_{i}$, we change the rationalizing utility function of Theorem 3.5 (b) into

$$
f(x)=\min _{i \in I} \lambda_{i} \hat{c}_{i}(x) / c_{i}\left(x^{i}\right) \quad(x \in X)
$$

Then, analogously as is in the proof of Theorem 2.8 , we have $f \in \mathcal{F}_{d}\left(\Phi_{\mathrm{e}} D\right)$. Furthermore, $f$ is a continuous linearly homogeneous function, because all functions $\hat{c}_{i}$ are continuous and linearly homogeneous.

If we apply the efficiency transformation of Example 3.1 to price and quantity data we can even derive a concave continuous linearly homogeneous function.

Theorem 3.7: Suppose $D$ is a set of price and quantity data and $\Phi_{0}$ is the efficiency transformation of Example 3.1. If there is a linearly homogeneous function in $\mathcal{F}_{d}\left(\Phi_{E} D\right)$ then there exists a concave continuous linearly homogeneous function $f \in \mathcal{F}_{\mathrm{d}}\left(\Phi_{\mathrm{w}} D\right)$.

Proof: In this case all functions $\hat{c}_{i}$ in Theorem 3.7 are concave continuous linearly homogeneous. Hence the function $f \in \mathcal{F}_{d}\left(\Phi_{6} D\right)$ in the proof of Theorem 3.7 is a concave linearly homogeneous rationalizing utility function. In that case even an infinite data set is allowed, because the infimum over an arbitrary set of concave functions is a concave function.

Let us denote the efficiency transformations in Example 2.3 and Example 3.11 as $\Phi_{e}$ and $A_{e}$ respectively. If we are comparing efficiency upper bounds for both efficiency transformations, one may ask which of the following assumptions is stronger: (a) $f \in \mathcal{T}_{d}\left(\Phi_{e} D\right)$ or $(b) f \in \mathcal{F}_{d d}\left(\Lambda_{e} D\right)$. In the theorem below I show that both assumptions are equivalent to each other in case $f$ is a linearly homogeneous utility function. 
Theorem 3.8: Suppose $f: X \rightarrow \mathbb{R}$ and $c: X \rightarrow \mathbb{R}_{+}$are binearly homogeneous, $x^{*} \in X$, $c\left(x^{\prime}\right)>0$, and $X$ is a cone. Then for the efficiency transformations $\Phi_{e}$ and $A_{t}$ as given above, one has $\Phi_{e}\left(c, x^{*}\right) \in \mathcal{D}_{d}(f)$ if and only if $\Lambda_{e}\left(c, x^{\prime}\right) \in D_{d}(f)$.

Proof: Let us use the notation $\left(a, x^{*}\right)=\phi_{e}\left(c, x^{\prime}\right)$ and $\left(b, x^{*}\right)=\Lambda_{e}\left(c, x^{\prime}\right)$. So, we have

$$
\begin{aligned}
& a(x)= \begin{cases}c(x) & \left(x=x^{\prime}\right) \\
e^{-1} c(x) & \text { (otherwise) },\end{cases} \\
& b(x)= \begin{cases}c(x) & \left(x=\lambda x^{\prime}, \lambda \in \mathbb{R}\right), \\
e^{-1} c(x) & \text { (otherwise). }\end{cases}
\end{aligned}
$$

$(\leftarrow)$ The conditions for consumer demand are automatically satisfied for $\left(a, x^{\prime}\right)$ in the area

$$
\left\{x \in X \mid x=\lambda x^{\prime \prime}, \lambda<0\right\}
$$

because we have

$$
a\left(\lambda x^{\prime}\right)=\lambda a\left(x^{\prime \prime}\right)<\alpha\left(x^{\prime}\right)=c\left(x^{\prime}\right) \quad(\lambda<0),
$$

since $c$ is linearly homogeneous and $c\left(x^{\prime}\right)>0$.

For $x$ in the remaining area we have $b(x) \leq a(x)$, because

$$
c\left(\lambda x^{*}\right) \leq e^{-1} c\left(\lambda x^{\prime}\right) \quad(\lambda \geq 0) \text {. }
$$

Hence, for $x$ in the area where we have to show that the conditions for consumer demand are satisfied, we have $b(x) \leq a(x)$. This and $b\left(x^{\prime}\right)=a\left(x^{\prime}\right)$ imply
that we have

$$
(b, x) \in \mathcal{D}_{d}\left(f^{\prime}\right) \Rightarrow\left(a, x^{\prime}\right) \in \mathcal{D}_{d}(f)
$$

$(\Rightarrow)$ : Let $\left(a, x^{\prime}\right) \in D_{d}(f)$. Then $a\left(x^{\prime}\right)=c\left(x^{\prime}\right)>0$ implies that we have

$$
a(0)=0<a\left(x^{\prime \prime}\right) \Rightarrow f(0)=0<f\left(x^{\prime}\right) \text {. }
$$

So, because both $b$ and $f$ are linearly homogeneous and positive at $x^{\prime}$, we have

$$
b(x) \leq b\left(x^{\prime}\right) \Leftrightarrow f(x) \leq f\left(x^{\prime}\right) \quad\left(x=\lambda x^{\prime}, \lambda \in \mathbb{R}\right) .
$$

Hence, for $x=\lambda x^{\prime}, \lambda \in \mathbb{R}$, the conditions of consumer demand are automatically
satisfied. In the remaining area, we have

$$
b(x)=a(x) \quad\left(x \in X: x \neq \lambda x^{\prime} \text { for all } \lambda \in \mathbb{R}\right)
$$

This, together with $b\left(x^{\prime}\right)=a\left(x^{\prime}\right)$, imply that in the remaining area there is no difference between both functions $a$ and $b$ with respect to the conditions of
consumer demand. So, we have

$$
\left(a, x^{\prime}\right) \in D_{d}(f) \Rightarrow\left(b, x^{\prime}\right) \in D_{d}(f)
$$
Varian (1983) describes also necessary and sufficient conditions for the
existence of a tationalizing linearly homogeneous utility function. A
difference with the theorems in this chapter economical efficient consumer in this chapter is that Varian considers quantities. The theorems in the demand for linite data sets of prices and Varian"s results in two ways the current and the previous section generalize demand, which includes efficient is the extension of the results to constumer data as a special case. The second from finite to infinite data sets. 


\subsection{A Linearly Homogeneous Function for Dutch Consumer Data}

A few researchers tested whether consumption data satisfy the nonparametric hypothesis of homotheticity of the utillity function. Manser and McDonald (1988) found no violation of the homotheticity hypothesis for U.S. consumption data, and Diewert and Parkan (1978) found a slight violation of the homotheticity hypothesis for Canadian consumption clata. They use a measure for the violation of the homotheticity hypothesis that is not very easy to compute. Furthermore, their measure has not a clear economical interpretation.

We may use the efficiency transformation given in Example 3.1 to define an efficiency measure for price and quantity data. Using this efficiency transformation, we can compute the efficiency upper bound for the Dutch consumption data with respect to the family of homothetic utility functions. Using condition (iv) of Theorem 3.5 and a bisection algorithm, we can compute this upper bound, as is made clear in section 2.6. The result was $e=0.9996$ and the hypothesis of homotheticity is only slightly violated.

In addition to this, I determined the maximal subsets of observations, which are consistent with the homotheticity hypothesis at efficiency level one. To obtain consistency it was sufficient to delete only one observation, where the choice is to delete the observation for 1951 or, otherwise, the observation for 1952. By coincidence both these years are mentioned in Chapter 2, concerning the existence of a rationalizing utility functions, where we had to interchange them to obtain the lower triamgular matrix in Table 2.3.

\subsection{Restrictions on Linearly Homogeneous Utility Functions}

The nonparametric approach allows the derivation of an impression of consumers utility function without the need of a parametric specification of the utility function and estimation of the parameters. An example of this approach is given in Manser and Mcbonald (1988). They used the nomparametric theory of Afriat $(1972,1981)$ to compute nonparametric lower and upper bounds for linearly homogeneous utility function values for observed U.S. consumer data. They could compute these bounds, because the consumer data satisfied the hypothesis of efficient homothetic utility maximization. In other empirical studies, however, the homotheticity hypothesis is rejected. Diewert and Parkan (1978) found a slight violation of this hypothesis for the Canadian consumer data they considered, and a similar result is obtatined in the previous section for Dutch consumer data. So, often the homotheticity hypothesis is falsified, and one is not able to compute Afriat's nonparametric bounds.

In the remaining part of this chapter I generalize Afrats theory, that is concerned with efficient consumer demand for price and quantity data. An important result of the generalized theory is the existence of nonparametric lower and upper bounds for any giwen consumer data, provided one lowers the consumer's efficiency level sufficiently. I show how these nomparametric bounds may be constructed. Furthermore, I prove - under weak conditions that the nonparametric bounds are tationalizing utility functions for the consumer data.

To derive the restrictions on rationalizing linearly homogeneous utility functions $\mathbb{I}$ use the following definition.

Definition: Let $F$ be a collection of functions $f: X \rightarrow \mathbb{R}$. Then the relative lower bound $L_{/}$is defined as 


$$
L_{A}\left(F, x, x^{n}\right)=\inf _{f \in F} f(x) f f\left(x^{*}\right) \quad\left(x, x^{s} \in X\right)
$$

Similarly the relative upper boutd $U_{\gamma}$ is

$$
U_{N}\left(F, x, x^{\prime}\right) \equiv \sup _{f \in F} f(x) / f\left(x^{\prime \prime}\right) \quad\left(x, x^{\prime} \in X\right)
$$

First I derive two elementary theorems concerning relative bounds.

Theorem 3.9: Suppose $x^{i} \in X$ for $i=1,2$ and $F$ is a collection of functions $f: X \rightarrow \mathbb{R}$ such that $f\left(x^{i}\right)>0$ for $i=1,2$. Then

$$
L_{j}\left(f, x^{1}, x^{2}\right)=U_{\lambda}\left(F_{3}, x^{2}, x^{d}\right)^{-1}
$$

where we define $\infty^{-1}=0$.

Proof: One has

$$
\inf _{a \in A}=\left(\sup _{a \in A} a\right)^{-1} \quad(A \subset(0, \infty)) .
$$

One has the following theorem for relative upper bounds. A similar theorem, where less than or equal is replaced by larger than or equal, is valid for relative lower bounds.

Theorem 3.10: Suppose $x^{i} \in X$ for $i=1, \ldots, n$ and $F$ is a collection of functions $f: X \rightarrow \mathbb{R}$ such that $f\left(x^{i}\right)>0$ for $i=1, \ldots$, n. Then

$$
U_{N}\left(F, x^{0}, x^{n}\right) \leq \prod_{i=1}^{n} U,\left(F, x^{i-1}, x^{i}\right)
$$

Proof: One has

$$
f\left(x^{0}\right) / f\left(x^{n}\right)=\prod_{i=1}^{n} f\left(x^{i-1}\right) / f\left(x^{i}\right) \leq \prod_{i=1}^{n} U_{/}\left(F, x^{i-1}, x^{i}\right) \quad(f \in F) .
$$

\subsection{Construction of Nonparametric Bounds}

First I consider a simple data set which contains one element.

Theorem 3.11: Suppose $c: X \rightarrow \mathbb{R}_{+}$is a linearly homogeneous function, $x^{\prime \prime} \in X$, $c\left(x^{s}\right)>0$, and $X$ is a cone. Put

$$
F=\left\{f \in \mathcal{F}_{d}\left(\left\{\left(c, x^{*}\right)\right\}\right) \mid f \text { linearly homogeneows }\right\} .
$$

Then one has $U_{/}\left(F,{ }^{\prime}, x^{\prime}\right) \in F$ and

$$
U_{\lambda}\left(F, x, x^{\prime}\right)=c(x) / c\left(x^{\prime}\right) \quad(x \in X) .
$$

Proof: Put

$$
g(x)=c(x) / c\left(x^{*}\right) \quad(x \in X) .
$$

One can easily show that one has $g \in F$ and $g\left(x^{\prime}\right)=1$. Thus

$$
g(x) \leq U_{N}\left(F, x, x^{\prime}\right) \quad(x \in X) .
$$

Now, let $f$ be an arbitrary function in $F$. Then $f\left(x^{4}\right)>0$ by Theorem 3.4 . Furthermore, since $f$ and $c$ are linearly homogeneous, Theorem 3.3 implies

$$
f(x) / f\left(x^{\prime}\right) \leq c(x) / c\left(x^{+}\right)=g(x) \quad(x \in X) .
$$

Hence

$$
U_{/}\left(F, x, x^{*}\right) \leq g(x) \quad(x \in X)
$$

since $f$ was an arbitrary function in $F$. So, both inequalities imply $U_{x}\left(F, x, x^{*}\right)=g(x)$ for all $x \in X$.

The above example is simple. The aim is now to derive a similar result that applies to consumer data sets of an arbitrary number of elements. For this 
result we need the definition of the weak closire $A^{*}$ of a square matrix $A$, which is defined in Appendix $C$. The ldea of the meak closure is the following. Let the cost of a single step that noves from $i \in I$ to $j \in I$ be equal to $a_{i j}$. Then the weak closure $a_{i j}^{*}$, for $i, j \in I$, represents the cost of the cheapest path from to $j$. We may define the weak clostre as follows.

Definition: The weak closure $A^{*}$ of a matrix $A \in \mathbb{R}^{n} \times \mathbb{R}^{\text {nt }}$ defined as

$$
a_{i j}^{*}=\inf a_{i k}+a_{k l}+\cdots+a_{m n}+a_{n j}
$$

where the infimum is taken over all possible sequences $k, l, \ldots, m, n \in I$.

Using this definition, we formulate in the following theorem relative upper and lower bounds for linearly homogeneous consumer demand.

Theorem 3.12: Suppose $D=\left\{\left(c_{i}, x^{i}\right)\right\}_{i \in I}$ is a data set, where $c_{\mathrm{i}} \times X_{\rightarrow} \rightarrow \mathbb{R}_{+}$are linearly homogeneous and $c_{i}\left(x^{i}\right)>0$, for all $i \in I$, and $X$ is a cone. $P_{\text {at }}$

$$
\begin{aligned}
& F=\left\{f \in \mathcal{F}_{d}(D) \| f \text { linearly homogencous }\right\}, \\
& a_{i j}=\ln \left(c_{j}\left(x^{i}\right) / c_{j}\left(x^{j}\right)\right) \quad(i, j \in I) .
\end{aligned}
$$

If $F \neq \emptyset$ then one has $U,\left(F,{ }^{n} x^{j}\right) \in F$ for all $j \in I$, and

$$
\begin{aligned}
& U_{\lambda}\left(F, x, x^{j}\right)=\inf _{i \in I}\left[c_{i}(x) / c_{i}\left(x^{i}\right)\right] \mathrm{e}^{a_{i j}^{*}} \quad(j \in I, x \in X), \\
& U_{\lambda}\left(F, x^{i}, x^{j}\right)=\mathrm{e}^{a_{i j}^{*}}, U_{\lambda}\left(F, x^{i}, x^{j}\right)^{-1}=L_{/}\left(F, x^{j}, x^{i}\right) \quad(i, j \in I) .
\end{aligned}
$$

Proof: Suppose $F \neq \varnothing$. Then, by Theorem 3.11 and Theorem 3.5 , the matrix $A$ has to be absorptive. This means that the weak closure $A^{*}$ is well-defined and we have $a_{i j}^{*} \leq a_{i k}+a_{k j}^{*}$, or equivalently

$$
e^{a_{i j}^{*}} \leq\left[c_{k}\left(x^{i}\right) / c_{k}\left(x^{k}\right)\right] e^{a_{k j}^{*}} \quad(k, i, j \in I) \text {. }
$$

Let $j \in I$ and put

$$
g(x)=\inf _{i \in I}\left[c_{i}(x) / c_{i}\left(x^{i}\right)\right] \mathrm{e}^{a_{i j}^{*}} \quad(x \in X) .
$$

Hence, one has

$$
g\left(x^{i}\right)=\inf _{k \in I}\left[c_{k}\left(x^{i}\right) / c_{k}\left(x^{k}\right)\right] \mathrm{e}^{a_{k j}^{*}}=\mathrm{e}^{a_{i j}^{*}} \quad(i \in I) .
$$

To prove that one has $g \in F$, define

$$
f_{i}(x)=c_{i}(x) / c_{i}\left(x^{i}\right) \quad(i \in I, x \in X),
$$

and thus $f_{i} \in \mathcal{F}_{d}\left(\left\{\left(c_{i}, x^{i}\right)\right\}\right)$ by Theorem 3.11. So one has

$$
\begin{array}{lll}
c_{i}(x) \leq c_{i}\left(x^{i}\right) & \Rightarrow g(x) \leq f_{i}(x) \mathrm{e}^{a_{i j}^{*}} \leq f_{i}\left(x^{i}\right) \mathrm{e}^{a_{i j}^{*}}=g\left(x^{i}\right) & (i \in I, x \in X), \\
c_{i}(x)<c_{i}\left(x^{i}\right) \Rightarrow g(x) \leq f_{i}(x) \mathrm{e}^{a_{i j}}<f_{i}\left(x^{i}\right) \mathrm{e}^{a_{i j}^{*}}=g\left(x^{i}\right) & (i \in I, x \in X) .
\end{array}
$$

Hence $g \in \mathcal{F}_{d}(D)$, and because $g$ is obviously a linearly homogeneous function we have thus $g \in F$.

From $a_{j j}=0$ follows $a_{j j}^{*}=0$, because $A$ is absorptive. So one has

$$
g\left(x^{j}\right)=\mathrm{e}^{a_{j j}^{*}}=1
$$

which in conjunction with $g \in F$ means

$$
g(x)=g(x) / g_{j}\left(x^{j}\right) \leq U_{,}\left(F, x, x^{j}\right) \quad(x \in X) .
$$

Thus to prove $g(x)=U_{f}\left(F, x, x^{j}\right)$, it remains to show that $U_{f}\left(F, x, x^{j}\right) \leq g(x)$. Put $F_{i}=\left\{f \in \mathcal{F}_{\alpha}\left(\left\{\left(c_{i}, x^{i}\right)\right\}\right) \mid f\right.$ linearly homogeneous $\}$. 
One has clearly $F \subset F$, so that

$$
U_{1}\left(F, x, x^{i}\right) \leq U_{1}\left(F_{i}, x, x^{i}\right) \quad(i \in I, x \in X)
$$

Now, because Theorem 3.4 impiles $f\left(x^{*} ;>0\right.$, for all $i \in I$ and $f \in F$, one has by Theorem 3.10 that

$$
\begin{aligned}
& U_{f}\left(f, x, x_{m}^{i}\right) \leq U_{J}\left(F, x, x^{i} v\right) \prod_{n=1}^{m} U_{N}\left(F, x^{i} n-1, x^{i} n\right)
\end{aligned}
$$

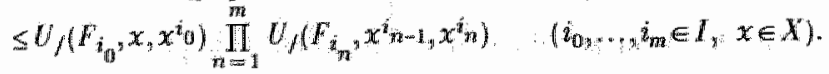

The later impities that we have

$$
v_{N}\left(F, x, x^{j}\right) \leq U_{N}\left(F_{i,}^{*} x, x^{i}\right) \mathrm{e}^{a_{i j}^{*}} \quad(i \in I, x \in X)
$$

Hence, since by Theorem 311 one has

$$
U_{/}\left(F_{i *}^{*}, x^{i}\right)=c_{i}(x) / c_{i}\left(x^{i}\right) \quad(i \in I, x \in X)
$$

we obtain

$$
U_{,}\left(F, x, x^{j}\right) \leq g(x) \quad(x \in X)
$$

which we had to show.

Finally, from Theorem 3.9 follows $L_{/}\left(F, x^{j}, x^{i}\right)=U_{,}\left(F, x^{i}, x^{j}\right)^{-11}$ for all $i, j \in l$.

Theorem 3.12 generalizes Afriat's theory in several ways. First, I have used a more general description of consumer behaviour. Secondly, the theorem is valid for infinite data sets. Thirdly, I have derived the upper bound $U_{,}\left(F, F_{2}, x^{j}\right)$ for any $x \in X$, whereas Afriat is only concened with the upper bounds $f_{A}\left(F^{i}, x^{i}, x^{j}\right)$, restricted to consumer's quantity choices.

Note that the lower bound $L_{f}\left(F, x, x^{j}\right)$ is not very interesting if $x \neq x^{i}$ for all $i \in l$. Then one has clearly $L /\left(F, x, x^{j}\right)=-\infty$. For a finite lower bound in this case one has to restrict $F$ to a smaller family of functions, for instance, the family of nonnegative linearly homogeneous functions.

Theorem 3.13: Sippose $D=\left\{\left(c_{i}, x^{i}\right)\right\}_{i \in \mathbb{I}}$ is a data set, where $c_{i}: X \rightarrow \mathbb{R}_{*}$ are linearly homogeneous and $c_{i}\left(x^{i}\right)>0$, for all $i \in I$, and $X$ is a cane. Pat

$$
F=\left\{f \in \mathcal{F}_{d}(D) \mid f \text { linearly homogeneous }\right\}, \bar{F}=\{f \in F \mid f \text { nonnegative }\} .
$$

Then one has:

$$
L_{/}\left(\vec{F}, x, x^{i}\right)=\max \left\{L /\left(F, x, x^{i}\right), 0\right\} \quad(i \in I, x \in X) .
$$

Proof: One can easily show that one has

$$
\overrightarrow{F^{2}}=\{g \mid f \in F, g(x)=\max \{f(x), 0\}\} \text {. }
$$

From this it is straightforward to derive the result.

Summaring the above we have derived the following result. For linearly homogeneous consumer demand there is a symmetry for the cost functions and the utility function. For the resulting consumer behaviour this symmetry implies a translation symmetry in the commodity space along the rays that have the origin as source. Using this translation symmetry we have derived an expression for the relative upper bound. This bound represents a conserved property that can be described as follows. Let $D$ be a set of data and let $\mathcal{L}$ denote the family of linearly homogeneous functions. Then we have

$$
f \in \mathcal{F}_{d}(D) \cap \mathcal{L} \Rightarrow f(x) / f\left(x^{\prime}\right) \leq V_{,}\left(\mathcal{F}_{d}\left(D^{\prime}\right) \cap \mathcal{L}, x, x^{\prime \prime}\right) \quad\left(f\left(x^{\prime}\right)>0, D^{\prime} \subset D\right) .
$$

The rellative upper bound $V_{f}\left(\mathcal{F}_{d}\left(D^{\prime}\right) \cap \mathcal{L}, x, x^{\prime}\right)$ is given in Theorem 3.12 . So, we have found a conserved property for linearly homogeneous consumer demand, because the right hand inequalities remain conserved when data is added to $D$. 
Remark: The above implication is a special case of the following result. For sets $F, F$ of functions one has in general

$$
f \in F \Rightarrow f(x) / f\left(x^{*}\right) \leq U,\left(F, x, x^{\prime}\right) \quad\left(f\left(x^{*}\right)>0, F \subset F^{*}\right)
$$

Note that $D^{\prime} \subset D$ implies $\mathcal{F}_{d}(D) \subset \mathcal{F}_{d}\left(D^{\prime}\right)$, because an increase in data information decreases the rationalizing set of functions, so that we have indeed

$$
\mathcal{F}_{d}(D) \cap \mathcal{L} \subset \mathcal{F} d\left(D^{\prime}\right) \cap \mathcal{L} \quad\left(D^{\prime} \in D\right)
$$

\subsection{Nonparametric Bounds for Dutch Consumer Data}

In this section the above theory is applied to the Dutch consumer data. The nonparametric lower and upper bounds are computed for rationalizing linearly homogeneous utility functions at efficiency level $e=0.9996$. This efficiency level is the efficiency upper bound for linearly homogeneous behaviour, we found earlier in this chapter. The first year 1951 is used as base year. As described in Theorem 3.12 , these nonparametric bounds follow from the shortest paths in a labelled graph with the adjacency matrix $\left.A=\llbracket a_{i j}\right]$, such that

$$
a_{i j}= \begin{cases}\lambda & \left(x^{i}=\lambda x^{j}, \lambda>0\right), \\ e^{-1} P_{i j} & \text { (otherwise), }\end{cases}
$$

where $P_{i j}=p^{j} x^{i} / p^{j} x^{j}$ is the direct Laspeyres index for current period and base period $j$. Because the Dutch consumption dlata have the property $x^{i} \neq \lambda x^{j}$ if $i \neq j$, as one may expect of empirical data, this matrix has the form

$$
a_{i j}= \begin{cases}1 & (i=j), \\ e^{-1} P_{i j} & \text { (otherwise) } .\end{cases}
$$

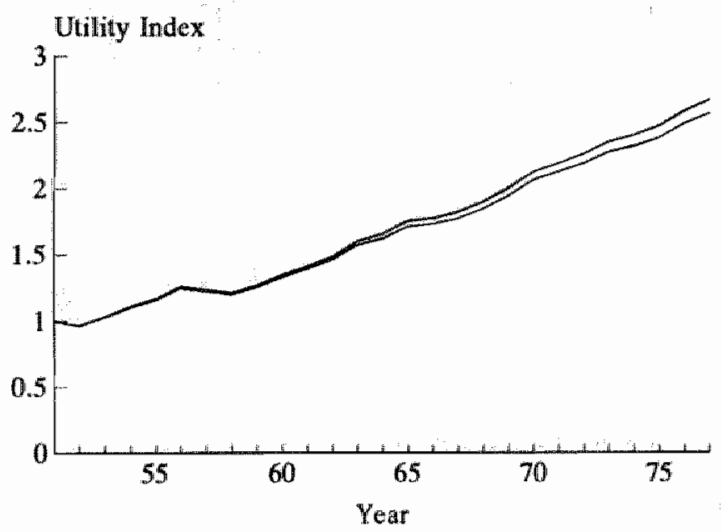

Figure 3.3 Bounds Linearly Homogeneous Utility

I computed the nonparametric lower and upper bounds for every year $i$ with

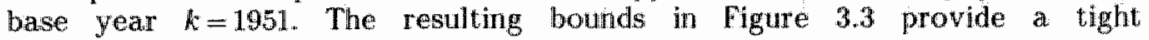


estmation for the willty values of a rationalizing linearly homogeneous utility function. By Theorem 3.12 these bounds are

$$
L_{y}\left(V_{,}, x^{i}, x^{k}\right)=\mathrm{e}^{-a_{k i}^{*}}, V_{N}\left(F, x^{i}, x^{k}\right)=\mathrm{e}^{a_{i k}^{*}}
$$

where $F$ is the family of rationalizing linearly homogeneous utility functions for data transformed using efficiency level $c$.

Table 3.1 Quantity Inclex Nunbers (base year 1951)

\begin{tabular}{|c|c|c|c|c|c|c|c|}
\hline \multicolumn{3}{|c|}{ Direct } & \multicolumn{2}{|c|}{ Chandined } & \multicolumn{2}{|c|}{ Nomparametric } & \\
\hline Year & Parasche & Laspeyres & Padsche & Laspeyres & Lower & Upper & \\
\hline 1951 & 1.000 & 1.000 & 1.000 & 1.000 & 1.000 & 1.000 & \\
\hline 1952 & 0.968 & 0.967 & 0.968 & 0.967 & 0.967 & 0.967 & \\
\hline 1953 & 1.031 & 1.034 & 1.030 & 1.032 & 1.031 & 1.033 & \\
\hline 1954 & 1.104 & 1.113 & 1.104 & 1. 109 & 1.104 & 1.110 & \\
\hline 1955 & 1.157 & 1.170 & 1.158 & 1.164 & 1.158 & 1.166 & 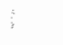 \\
\hline 1956 & 1.242 & 1.269 & 1.246 & 1.256 & 1.246 & 1.259 & \\
\hline 1957 & $\mathbb{1 . 2 1 9}$ & 1.250 & 1.227 & 1.239 & 1.227 & 1.240 & \\
\hline 1958 & 1.192 & $1.21 \pi$ & 1.202 & 1.215 & 1.202 & 1.214 & \\
\hline 1959 & 1.244 & 1.276 & 1.255 & 1.269 & 1.256 & 1.269 & \\
\hline 1960 & 1.311 & 1.359 & 1.328 & 1.344 & 1.328 & 1.344 & \\
\hline 1961 & 1.373 & 1.4 .33 & 1.394 & 1.412 & 1.394 & 1.413 & \\
\hline 1962 & 1.432 & 1.507 & 1.463 & 1.484 & 1.462 & 1.485 & \\
\hline 1963 & 1.534 & 1.631 & 1.573 & 1.597 & 1.572 & 1.599 & \\
\hline 1964 & 1.579 & 1.700 & 1.621 & 1.654 & 1.620 & 1.656 & \\
\hline 1965 & 1.655 & 1.803 & 1.711 & 1.748 & 1.709 & 1.752 & \\
\hline 1966 & 1.668 & 1.822 & 1.730 & 1.770 & 1.730 & 1.773 & \\
\hline 1967 & 1.706 & 1.865 & 1.773 & 1.815 & 1.772 & 1.820 & \\
\hline 1968 & $1.7 \mathrm{Tt}$ & 1.955 & 1.846 & 1.891 & 1.844 & 1.896 & \\
\hline 1969 & 1.850 & 2.069 & 1.942 & 1.992 & 1.940 & 1.998 & \\
\hline 1970 & 1.953 & 2.226 & 2.062 & 2.117 & 2.061 & 2.124 & \\
\hline 1971 & 2.014 & 2.288 & 2.120 & 2.179 & 2.122 & 2.186 & \\
\hline 1972 & 2.063 & 2.360 & 2.184 & 2.248 & 2.185 & 2.256 & \\
\hline 1973 & 2.140 & 2.459 & 2.272 & 2.339 & 2.271 & 2.349 & \\
\hline 1974 & 2.183 & 2.517 & 2.313 & 2.388 & 2.315 & 2.399 & \\
\hline 1975 & 2.240 & 2.601 & 2.377 & 2.456 & 2.379 & 2.468 & \\
\hline 1976 & 2.325 & 2.720 & 2.482 & 2.568 & 2.485 & 2.581 & \\
\hline 1977 & 2408 & 2.827 & 2.558 & 2.652 & 2.562 & 2.666 & \\
\hline
\end{tabular}

Table 3.1 compares the nonparametric lower and upper bounds for base year 1981 with direct and chained Paasche and Laspeyres quantity index numbers with base year 1951 .

As known, and as one can derive from the theory in this chapter, the Paasche and Laspeyres index numbers are lower and upper bounds of the nonparametric lower and upper bounds respectively, provided consumer behaviour is efficient and linearly homogeneous. However, since the consumption data was only nearly efficient linearly homogeneous, the Paasche and Laspeyres index numbers do in some cases slightly violate the nonparametric bounds. It is remarkable how close the chained Paasche and Laspeyres index numbers are to the nonparametric lower and upper bounds respectively, while this is not the case for the direct Pasche and Laspeyres index numbers.

In order to analyse the results for the nonparametric bounds, I investigated the shortest patllus for $a_{i j}^{*}$, from which these bounds follow. These paths are 
given in Table 3.2 and Table 3.3 .

It is striking that the sequences for the shortest paths are nearly all in time order except for the recession years 1956,1957 and 1958 for the upper bound computations. Especially because the method to derive the shortest paths is independent of the order of the observations.

Table 3.2 Shortest Paths for Computation Lower Bounds

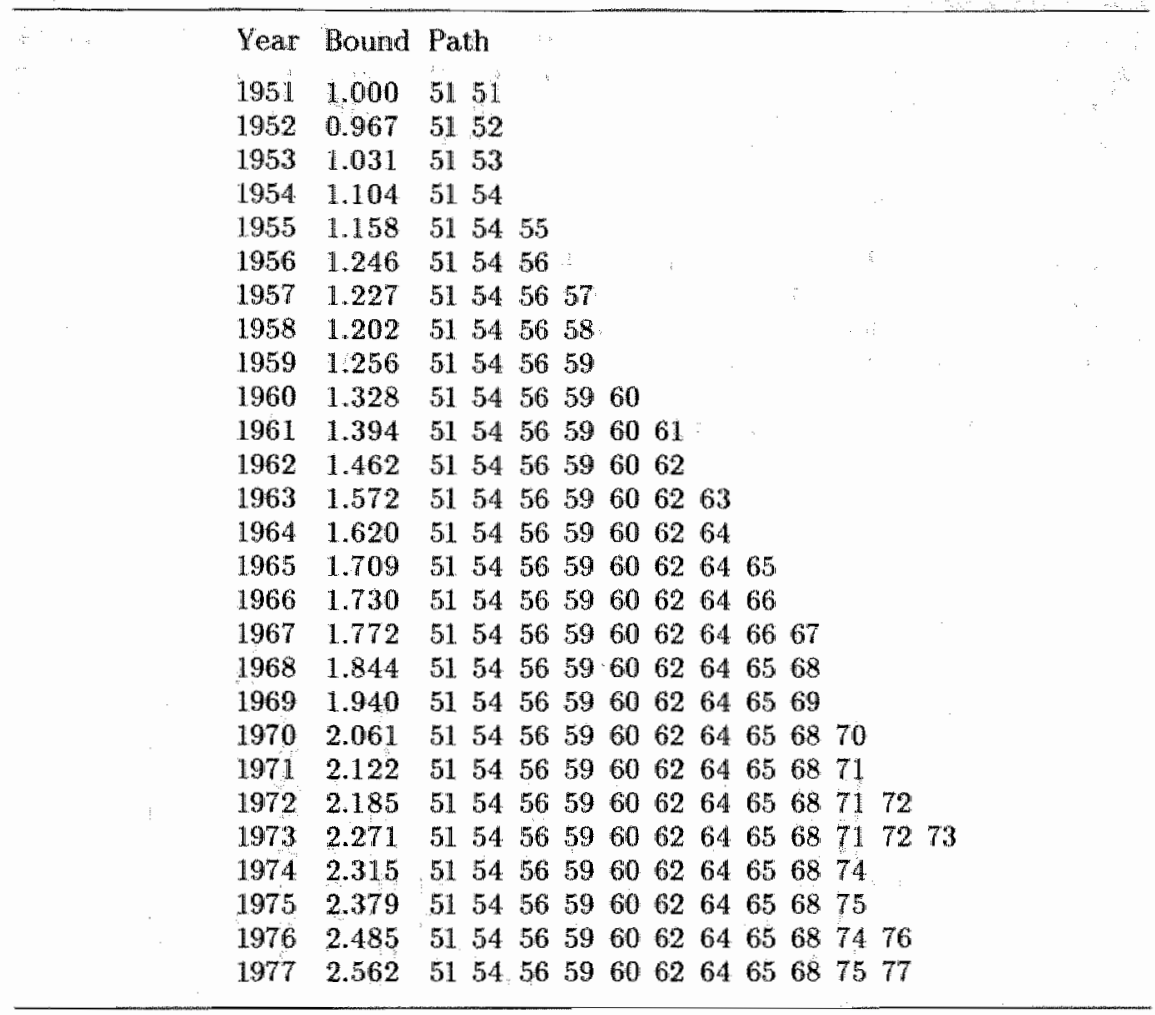

One might think this is caused by the increase of real income per capita over time. However, this is certainly not the case, because multiplication of the quantity vectors $x^{i}$ by arbitrary numbers $\gamma_{i}>0$ may change the nonparametric bounds for the data set, but will not affect the years which appear in Table 3.2 and 3.3 .

Remark: After multiplication of $x^{i}$ with $\gamma_{i}$, the new adjacency matrix $A^{\prime}=\left[a_{i j}^{u}\right]$ will be of the form

$$
a_{i j}=a_{i j}+\alpha_{i}-\alpha_{j}, \alpha_{i}=\ln \gamma_{i} .
$$

Now, the difference of the cost of any path $\mu$ from $i$ to $j$ at adjacency matrix $A$ and adjacency matrix $A$, respectively, will be $\alpha_{i}-\alpha_{j}$, because all $\alpha_{k}$ terms from years (vertices) in between will cancel each other out. Hence, the same years (vertices) appear in the shortest paths for both adjacency matrices $A$ and $A^{\prime}$, although the cost of the shortest paths may differ. So an increase of real income is no explanation for the resulting, shortest paths.

The reason why the shortest paths are in time order becomes clear by 
considering the nonparametric upper bound

$$
U_{/}\left(F, x_{*} x^{j}\right)=\min _{i}\left[c_{i}(x) / c_{i}\left(x^{i}\right)\right] \mathrm{e}^{a_{i j}^{*}}
$$

given in Theorem 3.112, where

$$
\begin{aligned}
& F=\left\{f \mid f \in \mathcal{F}_{d}\left(\left\{\left(c_{i}, x^{i}\right)\right\}_{i \in I}\right), f \text { linearly homogeneous }\right\}, \\
& \left(c_{i}, x^{i}\right)=\Phi_{e}\left(p^{i}, x^{i}\right) \quad(i \in I) .
\end{aligned}
$$

The reader may verify that a shortest path from $x^{k}$ to $x^{j}$ with cost $a_{k j}^{*}$ can be represented by a curve from $x^{k}$ to $x^{j}$ at the surface of the function $U_{,}\left(f, x, x^{i}\right)$. It visits the vertices $x^{i}$ on the shortest path from $x^{k}$ to $x^{j}$ and is a curve along the corresponding active constrairits

$$
\left[c_{i}(x) / c_{i}\left(x^{i}\right)\right] \mathrm{e}^{a_{i j}^{*}}
$$

that determine $U_{J}\left(F, x, x^{j}\right)$. We may scale all points $x$ on this curve, using a factor $\lambda(x)$ such that $\lambda(x)^{-1} x$ represents a indifference curve. The shortest paths will be in time order when the scaled quantities $\lambda\left(x^{i}\right)^{-1} x^{i}$ on such an indifference curve tend to move in the same direction when time increases.

Table 3.3 Shortest. Paths for Computation Upper Bounds

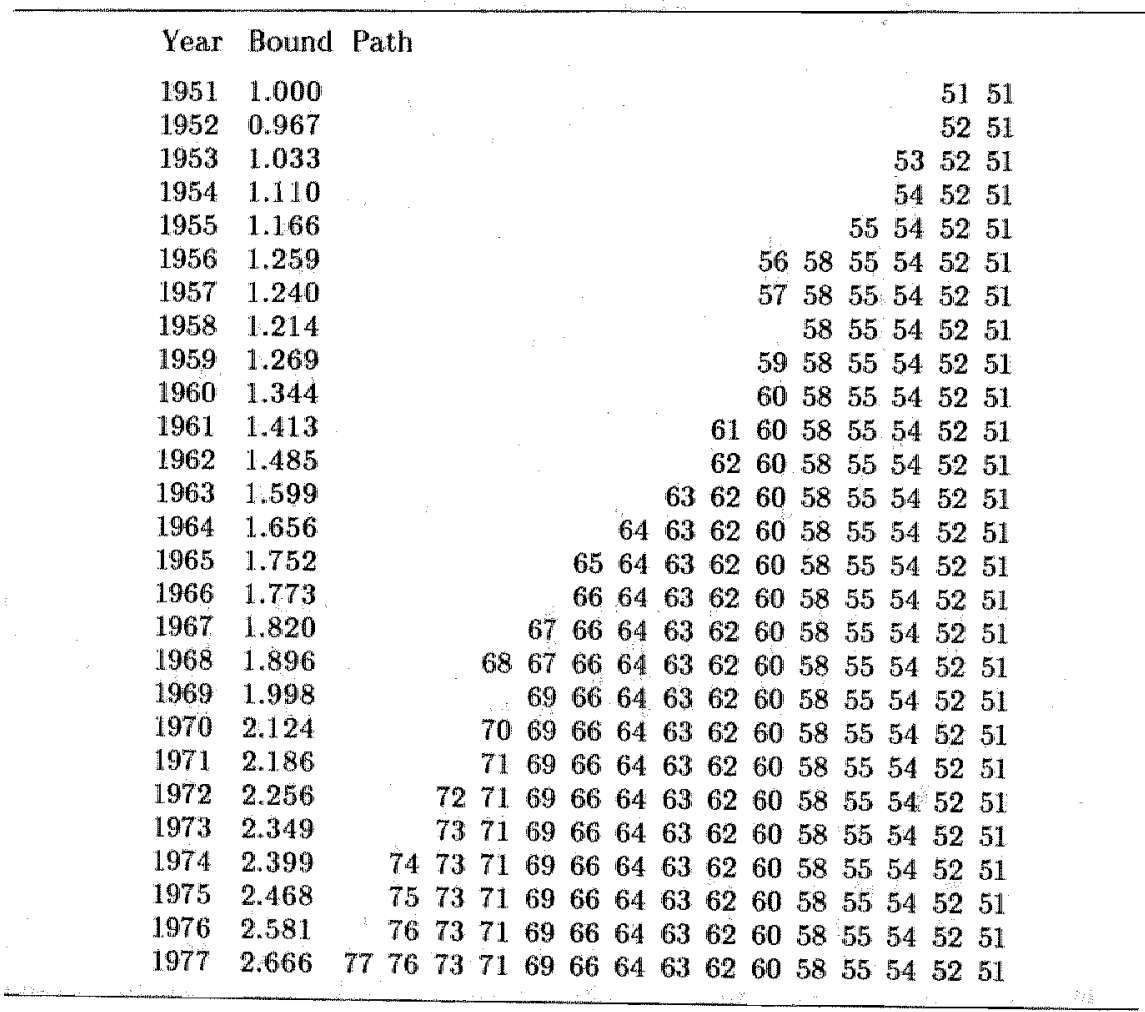

This phenomenon is illustrated in Figure 3.4 for a simple two-dimensional example of efficient consumer behaviour. When the rays, from the origin through the quantity observations, tend to move in a certain direction when time increases, then the shortest paths will tend to be in time order. This in turn, in conjunction with an efficiency level close to one, explains the 
resemblance of the claained Pasche and Laspeyres index numbers to the nonparametric lower and upper bounds respectiwely.

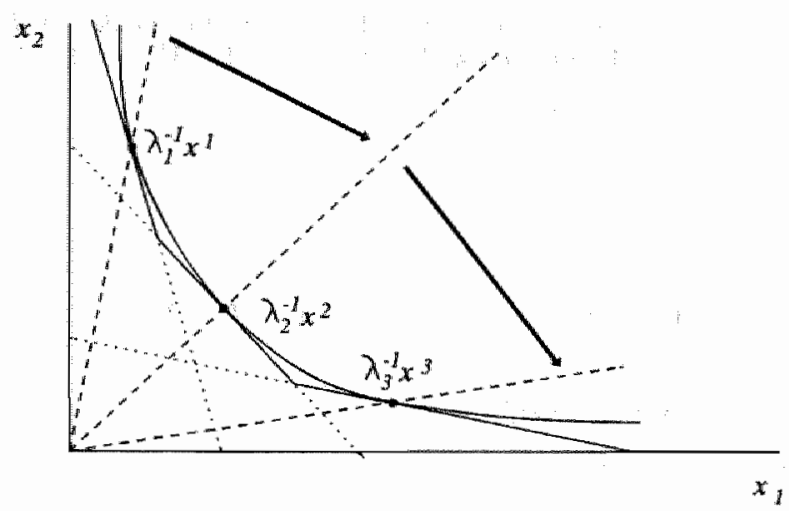

Figure 3.4 Linearly Homogeneous Demand

\subsection{Approximating Nomparametric Bounds for Concave Functions}

Unfortunately, when we consider concave linearly homogeneous functions, the nonparametric upper bound restrictions become more complicated. There is no problem if the nonparametric upper bound for all rationalizing linearly homogeneous functions is concave. Then this bound is equal to the upper bound for the subset of concave linearly homogeneous functions. However, it may happen that we do not obtain such a concave upper bound For instance, this is the case if price and quantity data is transformed, using an efficiency transformation. In that case we may try to approximate the upper bound for concave linearly homogeneous functions, using the nonparametric bounds for the case without the restriction of concavity. This approach is used in the following theorem.

Theorem 3.14: Suppose $D=\left\{\left(c_{i:} x^{i}\right)\right\}_{i \in I}$ is a data set, where $c_{i}: X \rightarrow \mathbb{R}_{*}$ are linearly homogeneous and $c_{i}\left(x^{i}\right)>0$, for all $i \in I$, and $X$ is a cone. Put

$$
\begin{aligned}
& F=\left\{f \in \mathcal{F}_{d}(D) \mid f \text { linearly homogeneous }\right\}, F=\{f \in F \mid f \text { concave }\} \\
& \hat{c}_{i}(x)=\lim _{x \rightarrow x} c_{i}(v) \quad(i \in I, x \in X)
\end{aligned}
$$

where we take the limit along $v \notin\left\{\lambda x^{i} \mid \lambda \in \mathbb{R}\right\}$. Suppose all functions $\hat{\mathbb{C}}_{i}$ are concave linearly homogeneous and

$$
\hat{c}_{i}(x) \geq c_{i}(x) \quad(i \in I, x \in X)
$$

Then the nonparametric bounds for $\bar{F}$ satisfy

$$
\begin{aligned}
& {\left[c_{j}\left(x^{j}\right) / \hat{c}_{j}\left(x^{j}\right)\right] U_{N}\left(F, x^{i}, x^{j}\right) \leq U_{N}\left(\bar{F}, x, x^{j}\right) \leq U_{N}\left(F, x, x^{j}\right) \quad(j \in I, x \in X)} \\
& U_{\lambda}\left(\bar{F}_{,} x^{i}, x^{j}\right)^{-1}=L_{\lambda}\left(\bar{F}, x^{j}, x^{i}\right) \quad(i, j \in I) .
\end{aligned}
$$

Proof: Put 


$$
a_{j}=\ln \left(c_{j}\left(x^{i}\right) / c_{j}\left(x^{j}\right)\right) \quad(l, j \in I)
$$

Moreover, let $j \in I$ and put

$$
\begin{array}{ll}
g(x)=\inf _{i \in I}\left[\hat{c}_{i}(x) / c_{i}\left(x^{i}\right)\right] e^{a_{i j}} & (x \in X), \\
g(x)=\inf _{i \in !}\left[c_{i}(x) / c_{i}\left(x^{i}\right)\right] e^{a_{i j}^{*}} & (x \in X)
\end{array}
$$

As is shown in the proof of Theorem 3.6 and using. Theorem 3.12, it follows that one has $g \in F, \hat{g} \in \bar{F}$, and $g \leq \hat{g}$. Furthermore, by Theorem 3.12 one has

$$
g(x)=U_{f}\left(F, x, x^{\prime}\right) \quad(x \in X)
$$

Now, because $\vec{F} \subset F$, we have

$$
U_{/}\left(\vec{F}, x, x^{j}\right) \leq U_{/}\left(F, x, x^{j}\right) \quad(x \in X)
$$

and thus

$$
g(x) / \hat{g}\left(x^{j}\right) \leq \hat{g}(x) / \hat{g}\left(x^{j}\right) \leq U /\left(F, x, x^{j}\right) \leq g(x) \quad(x \in X),
$$

where

$$
g(x)=U_{1}\left(f, x, x^{j}\right) \quad(x \in \mathbb{X}) .
$$

So the result follows from the inequality

$$
\hat{g}\left(x^{j}\right) \leq\left[\hat{c}_{j}\left(x^{j}\right) / c_{j}\left(x^{j}\right)\right] e^{a_{j j}^{*}}=\hat{c}_{j}\left(x^{j}\right) / c_{j}\left(x^{j}\right),
$$

that is obtained by using the definition of $\hat{g}$ and $a_{j j}^{*}=0$. Finally, as in the proof of Theorem 3.12 , it follows from Theorem 3.9 that

$$
L_{/}\left(\bar{F}, x^{j}, x^{i}\right)=U_{/}\left(\bar{F}^{i}, x^{i}, x^{j}\right)^{-1} \quad(i, j \in I) .
$$

In case the efficiency, transformation $A_{\varepsilon}$ given in Example 3.1 is applied to data $D$ with concave cost functions, then the above theorem yields the following simple estimation of the nonparametric upper bound belonging to the transformed data

$$
\left.e U_{\lambda}\left(F, x, x^{j}\right) \leq U_{/}\left(\vec{F}, x, x^{j}\right) \leq U_{/}\left(F, x, x^{j}\right) \quad \| j \in I, x \in X\right)
$$

where

$$
F=\left\{f \in \mathcal{F}_{d}\left(A_{e} D\right) \mid f \text { linearly homogeneous }\right\}, F=\{f \in F \mid f \text { concave }\} \text {. }
$$

This follows immediately from Theorem 3.14 , because we obtain in this theorem concave linearly homogeneous functions $\hat{c}_{i}$, such that

$$
\begin{aligned}
& \hat{c}_{i}(x) \geq c_{i}(x) \quad(i \in I, x \in X), \\
& c_{j}\left(x^{j}\right) / \hat{c}_{j}\left(x^{j}\right)=e \quad(j \in I) .
\end{aligned}
$$

\subsection{Rationalizing Nomparametric Bounds}

The following theorem shows, under weak conditions, that nomparametric lower and upper bounds of sets of rationalizing linearly homogeneous functions do rationalize consumer data.

Theorem 3.15: Suppose $D=\left\{\left(c_{i}, x^{i}\right)\right\}_{i \in I}$ is a data set where $c_{i}: X \rightarrow \mathbb{R}_{4}$ are linearly homogeneous and $c_{i}\left(x^{i}\right)>0$, for all $i \in I$, and $X$ is a cone. Put

$$
F=\left\{f \in F_{d}(D) \mid f \text { linearly homogeneous }\right\},
$$

where $\vec{F} \neq \emptyset$ an arbitrary subset of $F$. Then one has for all $j \in I$ :

(a) $L /\left(F^{2}, x^{j}\right) \in F$, if it is a welk-defined finite function.

(b) $U_{/}\left(\tilde{F}, x^{i}\right) \in F$.

Proof (a): Obviously $L_{\mu}\left(\bar{F}, ; x^{j}\right)$ is a linearly homogeneous function as the 
pointwise infirnm over limearly homogeneous functions of the fom

$$
\left\{f(\cdot) / f\left(x^{j}\right) \mid f \in \vec{F}\right\} \text {. }
$$

In Theorem 5.4, that is proved later on, it is shown that such a pointwise infimum over utility maximizing functions is a utility maximizing function itself, assuming that it is a well-defined finite function. So, because $f \in \mathcal{T}_{u}(D)$ implies clearly $f(\cdot) / f\left(x^{j}\right) \in \mathcal{F}_{u}(D)$, we have $L_{7}\left(\bar{F}_{*}, x^{j}\right) \in \mathcal{F}_{u}(D)$.

Now, it remains to prove that $L(F, F, x)$ is cost mimimizing function. From Theorem 3.4 we obtain $f\left(x^{i}\right)>0$ for all $f \in F$ and $i \in 1$. Hence, Theorem 3.3 implies

$$
c_{i}\left(x^{j}\right) f\left(x^{i}\right) / f\left(x^{j}\right) \geq c_{i}\left(x^{i}\right) \quad\left(f \in F_{,}, j \in I\right)
$$

so that

$$
L_{N}\left(\bar{F}, x^{i}, x^{j}\right) \geq c_{i}\left(x^{i}\right) / c_{i}\left(x^{j}\right)>0 \quad(i, j \in I) .
$$

Thus, since $L_{J}\left(\vec{F}, \cdots, x^{j}\right) \in \mathcal{F}_{u}(D)$, it follows from Theorem 3.2 that one has $L_{f}\left(\tilde{F}, \cdot x^{j}\right) \in F_{e}(D)$.

(b): The proof is similar to the proof of (a). We only have to show that every $U_{l}\left(\vec{F}_{2}, x^{j}\right)$ is a well-defined finite function. From Theorem 3.3 we obtain the inequality

$$
f(x) / f\left(x^{j}\right) \leq c_{j}(x) / c_{j}\left(x^{j}\right) \quad(f \in F, j \in I, x \in X) .
$$

Thus every $U_{N}\left(\bar{F}_{,}, x^{j}\right)$ is a well-defined finite function.

From the above theorem, we can derive a theorem concerning the norparametric lower bounds for concave linearly homogeneous functions.

Theorem 3.16: Suppose $D=\left\{\left(c_{i}, x^{i}\right)\right\}_{i \in I}$ is a data set, where $c_{i}: X \rightarrow \mathbb{R}_{*}$ are linearly homogeneous and $c_{i}\left(x^{i}\right)>0$, for all $i \in I$, and $X$ is a cone. Put

$$
F=\left\{f \in \mathcal{F}_{d}(D) \mid f \text { linearly homogeneous }\right\}_{,} \vec{F}=\{f \in F \mid f \text { nonnegative concave }\}
$$

where $\bar{F} \neq \emptyset$ is an arbitrary subset of $F$. Then $L,\left(\vec{F}, x^{j}\right) \in \vec{F}$ for all $j \in I$, and: (a) If $X$ is convex then

$$
L_{/}\left(\bar{F}, x, x^{j}\right)=\sup \left\{\sum_{i \in I} \lambda_{i} L_{f}\left(\tilde{F}, x^{i}, x^{j}\right) \mid \sum_{i \in I} \lambda_{i} x^{i} \leq x, \lambda_{i} \geq 0\right\} \quad(j \in I, x \in X),
$$

where only a finite number of $\lambda_{i}$ may be unequal to zero.

(b) If all functions $c_{i}$, $i \in I$, are concove then

$$
L_{/}\left(\bar{F}, x^{i}, x^{j}\right)=U /\left(F, x^{j}, x^{i}\right)^{-1} \quad(i, j \in I) .
$$

Proof: Let $j \in 1$. From Theorem $3.15(a)$ one obtains $L_{\gamma}\left(\tilde{F}, x^{\prime}\right) \in F$. Furthermore, $L_{l}\left(\bar{F}, x, x^{j}\right) \in \bar{F}$ as the pointwise infimum over functions in $\bar{F}$.

(a): Let $j \in I$ and put

$$
f(x)=\sup \left\{\sum_{i \in I} \lambda_{i} L /\left(\bar{F}, x^{i}, x^{j}\right) \mid \sum_{i \in I} \lambda_{i} x^{i} \leq x, \lambda_{i} \geq 0\right\} \quad(x \in X)
$$

where only a finite number of $\lambda_{i}$ may be unequal to zero. The function $L_{\mathcal{L}}\left(\bar{F}_{3}, x^{j}\right)$ is a nonnegative concave linearly homogeneous function on a convex cone $X$. Hence the definition of $f$ implies

$$
\begin{array}{lc}
L_{f}\left(\bar{F}, x, x^{j}\right) \geq f(x) & (x \in X), \\
f\left(x^{i}\right)=L_{\gamma}\left(\bar{F}, x^{j}, x^{j}\right) & (i \in I) .
\end{array}
$$

Thus obviously $f \in \bar{F}$, since $L_{\gamma}\left(\vec{F}, " x^{j}\right) \in \bar{F}$. Now, this implies

$$
L_{/}\left(\bar{F}^{i}, x, x^{j}\right) \leq f(x) / f\left(x^{j}\right)=f(x) / L /\left(\tilde{F}, x^{j}, x^{j}\right)=f(x) \quad(x \in X),
$$

so we have $L /\left(\bar{F}, x, x^{j}\right)=f(x)$ for all $x \in X$.

(b): Follows immediately from Theorem 3.14. 


\section{WEAKLY SEPARABLE CONSUMER DEMAND}

\subsection{Introduction}

In empirical studies there is an extensive use of weakly separable utility functions. In literature there are many examples which study the local properties of these functions. For an encyclopedic discussion of separabllity, see Blackorby et al. (1979). However, there only are few theoretical results, concerning weakly separable functions, based on a revealed preference approach. Some results in this area may be found in Djewert and Parkan (1978, 1985), and Varian (1982), who consider only concave weakly separable utility functions. This chapter considers weakly separable utility functions in general. Although it will be pointed out how one may apply the theory to data, the chapter contains no application to the Dutch consumer data.

\subsection{Weakly Separable Utility Functions}

In this book I will use the following definition of weak separability.

Definition: We say that a function $f: X_{1} \times X_{2} \rightarrow \mathbb{R}$ is weakly separable in $X_{2}$ if there exists a function $h: X_{2} \rightarrow \mathbb{R}$ such that $f$ can be written in the form

$$
f\left(x_{1}, x_{2}\right)=g\left(x_{1}, h\left(x_{2}\right)\right) \quad\left(x_{1} \in X_{1}, x_{2} \in X_{2}\right) .
$$

The function $h$ is called a subfunction and $g$ an aggregator function.

Remark: In literature one assumes often that the aggregator function $g\left(x_{1}, \eta\right)$ is strictly increasing in its second argument:

If rational demand is generated by a weakly separable utility function $g\left(x_{1} h\left(x_{2}\right)\right)$, such that $g$ is strictly increasing in its second argument, then its subfunction is also compatible with rational demand, as is stated in the following theorem.

Theorem 4.1: Suppose $f(x)=g\left(x_{1}, h\left(x_{2}\right)\right)$ is weakly separable and $g\left(x_{1}, \eta\right)$ is strictly increasing in $\eta$. If $\left(c, x^{\prime}\right) \in \mathcal{D}_{d}(f)$ then $\left(\bar{c}_{,}, x_{2}\right) \in \mathcal{D}_{d}(h)$, where $\bar{c}(\cdot)=c\left(x_{i}^{*}, *\right)$.

Proor: Suppose $\bar{c}\left(x_{2}\right)<\bar{c}\left(x_{2}^{\prime}\right)$ for arbitrary $x_{2}$. Then $\left(c, x^{\prime}\right) \in \mathcal{D}_{u}(f)$ implies

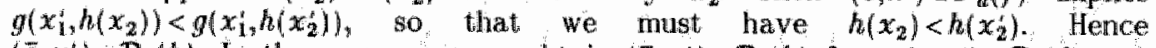
$\left(\bar{c}, x_{2}^{\prime}\right) \in D_{w}(h)$. In the same way we obtain $\left(\bar{c}_{,}, x_{2}^{\prime}\right) \in \mathcal{D}_{c}(h)$ from $\left(c, x^{*}\right) \in \mathcal{D}_{c}(f)$.

In terms of symmetry we have the following alternative definition of weak separabillity.

Theorem 4.2: A function is weakly separable of the form $f(x)=g\left(x_{1,} h\left(x_{2}\right)\right)$ if and only if $\boldsymbol{T}_{\mathrm{h}} f=f$, where

$$
\left(T_{h} f\right)\left(x_{1}, x_{2}\right)=\inf _{h(w)=h\left(x_{2}\right)} f\left(x_{1}, v\right) .
$$

Proof: This follows by using the aggregator function

$$
g\left(x_{i}, \eta\right)=\inf _{n(w)=\eta \eta} f\left(x_{1}, v\right)
$$

If the aggregator function $g\left(x_{1}, \eta\right)$ is increasing in the second argument then the weakly separable function satisfies the following symmetry.

Theorem 4.3: A function $f(x)=g\left(x_{1}, h\left(x_{2}\right)\right)$ is weakly separable such that the 
aggregator function $g\left(x_{1}, \eta\right)$ is increasing in $\eta$, if and only $\boldsymbol{T}_{h,} f=f$, where

$$
\left(T_{h} f\right)\left(x_{1}, x_{2}\right)=\inf _{h(v) \geq h\left(x_{2}\right)} f\left(x_{1}, v\right) .
$$

Proof: Use the aggregator function

$$
g\left(x_{1}, \eta\right)=\inf _{h(v) \geq \eta} f\left(x_{\mathbb{1}, q} v\right)
$$

One may use the abowe transformations $T_{h}$ to construct weakly separable function $T_{h} f$ from an arbitrary function $f$. The result is a weakly separable function, because obviously the following theorem holds.

Theorem 4.4: The above transformations $T_{h}$ satisfy $T_{k} T_{k}=T_{k}$.

Proof: Obvions.

The underlying idea of the theory in this chapter is the following. Consider the optimization problem

$$
\sup _{c(x) \leq c\left(x^{\prime \prime}\right)} f(x)
$$

for a given $x^{*}$. Using a Lagrangian, this problem can be transformed to an unconstrained optimization problem

$$
\sup _{x} f(x)-\lambda\left[c(x)-c\left(x^{\circ}\right)\right]
$$

where $\lambda>0$. Now, suppose $\boldsymbol{T}_{h} f=f$ holds, where $\boldsymbol{T}_{h}$ is one of the above transformations. Then we can show that

$$
\sup _{x} f(x)-\lambda\left[c(x)-c\left(x^{*}\right)\right]=\sup _{x} f(x)-\lambda\left[\left(T_{h} c\right)(x)-c\left(x^{\prime}\right)\right]
$$

holds. Furthermore, if we suppose that $x^{n}$ is a cost minimizing solution of the above optimization problem then $c\left(x^{4}\right)=\left(T_{h} c\right)\left(x^{n}\right)$, as is shown in the following theorem.

Theorem 4.5: Suppose $T_{h}$ is one of the transformations given above, i.e.

$$
\left(T_{h} f\right)\left(x_{1}, x_{2}\right)=\inf _{h(v)=h\left(x_{2}\right)} f\left(x_{1}, v\right) \text { or } \inf _{h(v) \geq h\left(x_{2}\right)} f\left(x_{1}, v\right) \text {. }
$$

If $\left(c, x^{\prime \prime}\right) \in \mathcal{D}_{c}(f)$ and $T_{h} f=f$ then $\left(T_{h} c\right)\left(x^{\prime \prime}\right)=c\left(x^{\prime}\right)$.

Proof: This follows from Theorem 4.2 and 4.3 . If $\boldsymbol{T}_{h} f=f$, we can write $f(x)=g\left(x_{1}, h\left(x_{2}\right)\right)$. Now, suppose $h(v)=h\left(x_{2}^{\prime}\right)$ for arbitrary $v$. Then $g\left(x_{1}^{\prime}, h(v)\right)=g\left(x_{1}^{\prime}, h\left(x_{2}^{\prime}\right)\right)$ and $\left(c, x^{\prime}\right) \in \mathcal{D}_{c}(f)$ implies thus $c\left(x_{1}^{\prime}, v\right) \geq c\left(x^{\prime}\right)$.

Next, suppose $g\left(x_{1}, \eta\right)$ is increasing in $\eta$ and $h(v) \geq h\left(x_{2}^{\prime}\right)$. Then we have $g\left(x_{1}^{\prime \prime}, h(v)\right) \geq g\left(x_{1}^{\prime}, h\left(x_{2}^{\prime \prime}\right)\right)$ and $\left(c, x^{\prime}\right) \in \mathcal{D}_{c}(f)$ implies again $c\left(x_{1}^{\prime}, v\right) \geq c\left(x^{\prime}\right)$.

The above theorem shows again that a symmetry $f=T_{h} f$ for the utility function can be translated in a symmetry for the corresponding consumer demand data. 'The symmetry $\left(\boldsymbol{T}_{h} c\right)\left(\boldsymbol{x}^{\prime}\right)=c\left(x^{\prime}\right)$ is a necessary condition for the existence of a rationalizing symmetric function $f=T_{h} f$.

So, concerning consumer demand for a weakly separable utility function, we can transform the original optimization problem into a problem for which the Lagrangian is of the symmetric form

$$
g\left(x_{1}, h\left(x_{2}\right)\right)-\lambda\left[\left(T_{h} c\right)(x)-\left(T_{h} c\right)\left(x^{\prime}\right)\right] .
$$

This observation leads to necessary and sufficient conditions for weakly separable consumer dernand. 


\subsection{Existence of a Rationalizing Aggregator Function}

Suppose a giwen set of consumer data on $X_{1} \times X_{2}$ and a given function $h: X_{2} \rightarrow R_{\text {R }}$. Then we may investigate whether there is an aggregator function $g\left(x_{1}, h\left(x_{2}\right)\right)$, which rationalizes the consuner data. In the following theorem a dual approach is used to construct such a rationalizing aggregator function $g$ from cost functions. Although only Theorem 4.6 (a) and (b) will be used later on, I give also Theorem $4.6(\mathrm{c})$ in order to show when the necessary condition in $(b)$ is a sufficient condition.

Theorem 4.6: Suppose $D=\left\{\left(c_{i}, x^{i}\right)\right\}_{i \in I}$ is a fimite data set, where $c_{i} X_{1} \times X_{2} \rightarrow \mathbb{R}_{*}$. Suppose $h: X_{2} \rightarrow \mathbb{R}$ and let the transformation $T_{h}$ of any function $f: X_{1} \times X_{2} \rightarrow \mathbb{R}$ be defined by

$$
\left(T_{H} f\right)\left(x_{1}, x_{2}\right)=\operatorname{linf}_{h(v) \geq h\left(x_{2}\right)} f\left(x_{1}, v\right) \quad\left(x \in X_{1} \times X_{2}\right)
$$

Suppose $\left(\boldsymbol{T}_{h} c_{i}\right)\left(x^{i}\right)=c_{i}\left(x^{i}\right)$, for all $i \in I$, and put $D^{*}=\left\{\left(\boldsymbol{T}_{h} c_{i}, x^{i}\right)\right\}_{i \in I^{*}}$.

(a) Then $\mathcal{F}_{d}\left(D^{\prime}\right) \subset \mathcal{X}_{d}(D)$.

(b) If $\mathcal{F}_{d}\left(D^{\prime}\right) \neq \emptyset$ then there exists a function $f \in \mathcal{F}_{d}(D)$ such that $T_{h} f=f$.

(c) Suppose $\boldsymbol{T}_{\mathrm{u}} f=f$ and suppose that the infima for each transformation $\boldsymbol{T}_{h} c_{i}$ are attained, i.e. for every $i \in I$ and $x \in X$ there is $a v \in X_{2}$ such that

$$
\left(T_{h i} c_{i}\right)(x)=c_{i}\left(x_{1}, v\right), h(w) \geq h\left(x_{2}\right) \text {. }
$$

Then $f \in \mathcal{F}_{d}(D)$ if and only if $f \in \mathcal{F}_{d}\left(D^{\prime}\right)$.

Proof (a): By definition of $T_{h}$, we have $T_{h} c_{i} \leq c_{i}$. Hence, using $\left(T_{i} c_{i}\right)\left(x^{i}\right)=c_{i}\left(x^{i}\right)$, we have

$$
\begin{array}{ll}
c_{i}(x) \leq c_{i}\left(x^{i}\right) \Rightarrow\left(\boldsymbol{T}_{h} c_{i}\right)(x) \leq\left(\boldsymbol{T}_{h} c_{i}\right)\left(x^{i}\right) & (x \in X, i \in I), \\
c_{i}(x)<c_{i}\left(x^{i}\right) \Rightarrow\left(\boldsymbol{T}_{h} c_{i}\right)(x)<\left(\boldsymbol{T}_{h} c_{i}\right)\left(x^{i}\right) & (x \in X, i \in I) .
\end{array}
$$

So $f \in \mathcal{F}_{d}\left(D^{\prime}\right)$ implies $f \in \mathcal{F}_{d}(D)$, and thus $\mathcal{F}_{d}\left(D^{*}\right) \subset \mathcal{F}_{d}(D)$.

(b): If $\mathcal{F}_{d}\left(D^{\prime}\right) \neq \emptyset$ then by Theorem 2.5 we can construct a function $f \in \mathcal{F}_{d}\left(D^{\prime}\right)$ of the form

$$
f(x)=\min _{i \in I} f_{i}+\lambda_{i}\left[\left(T_{h} c_{i}\right)(x)-\left(T_{h} c_{i}\right)\left(x^{i}\right)\right] \quad\left(x \in X_{1} \times X_{2}\right),
$$

such that $\lambda_{i}>0$ and $f\left(x^{i}\right)=f_{i}$. This function satisfies $\boldsymbol{T}_{h} f=f \in \mathcal{F}_{d}\left(D^{\prime}\right) \subset \mathcal{F}_{d}(D)$.

(c): Suppose $T_{k} f=f \in \mathcal{F}_{d}(D)$. Because of (a) it is sufficient to prove that one has now $f \in \mathcal{F}_{d}\left(D^{\prime}\right)$. Let $x \in X$ and $i \in I$ and suppose $\left(\boldsymbol{T}_{k} c_{i}\right)(x) \leq\left(\boldsymbol{T}_{k} c_{i}\right)\left(x^{i}\right)$. Then by
assumption there is a. $v \in X_{2}$ such that

$$
\left(T_{h} c_{i}\right)(x)=c_{i}\left(x_{1}, v\right), h(v) \geq h\left(x_{2}\right)
$$
Hence, we have $f(x) \leq f\left(x_{1}, v\right)$ and $c_{i}\left(x_{1}, v\right) \leq c_{i}\left(x^{i}\right)$. Moreover, $f \in \mathcal{F}_{d}(D)$ implies
$f\left(x_{1}, v\right) \leq f\left(x^{i}\right)$. So, we obtain

$$
\left(T_{h} c_{i}\right)(x) \leq\left(T_{k} c_{i}\right)\left(x^{i}\right) \Rightarrow f(x) \leq f\left(x^{i}\right) \text {. }
$$

Now, suppose $\left(\boldsymbol{T}_{h} c_{i}\right)(x)<\left(\boldsymbol{T}_{h} c_{i}\right)\left(x^{i}\right)$. Then in general there is a $v \in X_{2}$ such that

$$
\left(T_{i, c_{i}}\right)(x) \leq c_{i}\left(x_{1}, v\right)<\left(T_{h} c_{i}\right)\left(x^{i}\right), h(v) \geq h\left(x_{2}\right)
$$

Thus, similarly as above, we obtain

$$
\left(T_{k} c_{i}\right)(x)<\left(T_{h} c_{i}\right)\left(x^{i}\right) \Rightarrow f(x)<f\left(x^{i}\right)
$$

which means that we have $f \in \mathcal{F}_{d}\left(D^{\prime}\right)$.

So by Theorem 4.6 (c) the information concerning the possible symmetric functions $f=T_{h} f$ in $\mathcal{F}_{d}(D)$ is under weak conditions invariant under the data
transformation $D \rightarrow D^{\prime}$. 
Note that we can also prove the above theorem, using the transformation

$$
\left(T_{h} f\right)\left(x_{1}, x_{2}\right)=\inf _{h(v)=h\left(x_{2}\right)} f\left(x_{1}, v\right)
$$

In that case we consider weakly separable functions $g\left(x_{1}, h\left(x_{2}\right)\right)$ without the requirement that the aggregator function $g(x, m)$ is increasing in $n$.

\subsection{Existence of a Rationalizing Weakly Separable Function}

This section states conditions that are satisfied by weakly separable rational demand. In the previous section a rationalizing aggregator function $g$ is constructed for a given subfunction $h$. This was no problem, because the family of weakly separable utility function for a given subfunction $h$ can be described by using a symmetry. As is shown in the previous sections, there is a transformation $T_{h}$ that leaves such a family invariant. Further, as we have seen above and in Chapter 3 for linearly homogeneous consumer demand, a symmetry of the Lagrange function implies a symmetry for the corresponding consumer data. Moreover, it implies the existence of conserved properties, which can be used to gain information concerning the utility function.

The case of weak separabillity, however, differs from the problem in which linear homogeneity is considered. For every subfunction th there is another transformation $\boldsymbol{T}_{h}$ that leaves the weakly separable utility function invariant. So, if $h$ is not known then we do not know the symmetric property in advance. Furthermore, the assumption that the aggregator function $g\left(x_{1}, \eta\right)$ is strictly increasing in $\eta$ can not be derived from a symmetry assumption. This is why there is no simple solution for the construction of a complete rationalizing weakly separable function.

With some efforts I succeeded to derive results for weakly separable consumer demand, using the theory given in the prewious section. The following theorem is based on the construction of a suitable subfunction $h$, that is constructed in such a way that Theorem 4.6 (b) can be applied to derive a suitable aggregator function.

Theorem 4.7: Suppose $D=\left\{\left(c_{i}, x^{i}\right)\right\}_{i \in I}$, where $c_{i}: X_{1} \times X_{2} \rightarrow \mathbb{R}_{+}$, is a finite data set of consumer behaviour. Consider the following conditions:

(i) There exists a function $g\left(x_{1}, h\left(x_{2}\right)\right) \in \mathcal{F}_{d}(D)$, which is weakly separable in $X_{2}$ and such that $g\left(x_{1}, \eta\right)$ is increasing in $\eta$.

(ii) There exists a function $g\left(x_{1}, h\left(x_{2}\right)\right) \in \mathcal{F}$ d $(D)$, which is weakly separable in $X_{2}$ and such that $g\left(x_{1}, \eta\right)$ is strictly increasing in $\eta$.

(iii) There exist preorders $>\sim$ on $\left\{x^{i}\right\}_{\text {iel }}$ and $\succ$, $\sim$ on $\left\{x_{2}^{i}\right\}_{i \in I}$ such that

$$
\begin{aligned}
c_{i}\left(x_{1}^{j}, x_{2}^{k}\right) \leq c_{1}\left(x^{i}\right) \Rightarrow & \left(x^{j} \precsim x^{i} \text { or } x_{2}^{k}<^{k} x_{2}^{j}\right) \text { and } \\
& \left(x^{j} \prec x^{i} \text { or } x_{2}^{k} \aleph^{i} x_{2}^{j}\right) \quad(i, j, k \in I), \\
c_{1}\left(x_{1}^{j}, x_{2}^{k}\right)<c_{1}\left(x^{i}\right) \Rightarrow & x^{j}<x^{i} \text { or } x_{2}^{k} \prec^{i} x_{2}^{j} \quad(i, j, k \in I) .
\end{aligned}
$$

(iv) There exists a weakly separable function $g\left(x_{1}, h\left(x_{2}\right)\right) \in \mathcal{F}_{d}(D)$, such that $h$ and $g$ are strictly monotonically increasing continuous functions, and furtherrore $h$ and $g\left(\cdot h\left(x_{2}\right)\right)$, for every fixed $x_{2} \in X_{2}$, are concove.

Then:

(a) (ii) $\Rightarrow$ (iii) $\Rightarrow$ (i).

(b) Suppose every function

$$
h\left(x_{2}\right)=\min _{i, j \in I} \alpha_{i j}+\lambda_{i} c_{i}\left(x_{1}^{j}, x_{2}\right) \quad\left(x_{2} \in X_{2}\right),
$$

where $\alpha_{i j} \in \mathbb{R}$ and $\lambda_{i}>0$, is such that the functions 


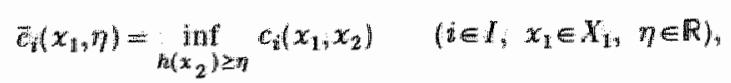

are strictly increasing in $\eta$. Then (iii) $\Leftrightarrow$ (ii).

(c) Sitppose $D$ is a price and quantity data set. Then (ii) $\leftrightarrow$ (iii) $\Leftrightarrow$ (jv).

Proof (a) (ii) $\Rightarrow$ (iii): Suppose $f(x)=g\left(x_{1}, h\left(x_{2}\right)\right) \in \mathcal{F}_{d}(D)$ is weakly separable and $g\left(x_{1}, \eta\right)$ is strictly increasing in $\eta$. Put $y_{i}=f\left(x^{2}\right)$ for all $i \in l$. Let $i, j, k \in I$ and suppose $c_{i}\left(x_{1}^{j}, x_{2}^{k}\right) \leq c_{i}\left(x^{2}\right)$. Then $f \in \mathcal{F}_{d}(D)$ implies

$$
g\left(x_{1}^{j}, h\left(x_{2}^{k}\right)\right) \leq g\left(x_{1}^{i}, h\left(x_{2}^{i}\right)\right)
$$

and $g$ strictly increasing in th, means

$$
\begin{aligned}
& g\left(x_{1}^{j}, h\left(x_{2}^{j}\right)\right)>g\left(x_{1}^{i}, h\left(x_{2}^{i}\right)\right) \Rightarrow g\left(x_{1}^{j} h\left(x_{2}^{j}\right)\right)>g\left(x_{1}^{j}, h\left(x_{2}^{k}\right)\right) \Rightarrow h\left(x_{2}^{j}\right)>h\left(x_{2}^{k}\right), \\
& g\left(x_{1}^{j}, h\left(x_{2}^{j}\right)\right) \geq g\left(x_{1}^{i} h\left(x_{2}^{i}\right)\right) \Rightarrow g\left(x_{1}^{j}, h\left(x_{2}^{j}\right)\right) \geq g\left(x_{1}^{j}, h\left(x_{2}^{k}\right)\right) \Rightarrow h\left(x_{2}^{j}\right) \geq h\left(x_{2}^{k}\right) .
\end{aligned}
$$

Hence, one has then

$$
\left(y_{j} \leq y_{i} \text { or } h\left(x_{2}^{k}\right)<h\left(x_{2}^{j}\right)\right) \text { and }\left(y_{j}<y_{i} \text { or } h\left(x_{2}^{k}\right) \leq h\left(x_{2}^{j}\right)\right) \text {. }
$$

Now, suppose $c_{i}\left(x_{2}^{j}, x_{2}^{k}\right)<c_{i}\left(x^{i}\right)$. Then it follows in a similar way that one has

$$
y_{j}<y_{i} \text { or } h\left(x_{2}^{k}\right)<h\left(x_{2}^{j}\right)
$$

So, let $\succ, \sim$ be the weak order on $\left\{x^{i}\right\}_{i \in I}$ induced by $f$, and let $\succ^{\prime}, \sim$ be the weak order on $\left\{x_{2}\right\}_{i \in I}$ induced by $h_{*}$. Then, by the above, these weak orders do satisfy (ini).

(iii) $\Rightarrow$ (i): If there are preorders satisfying the conditions in (iii), then by Theorem A.2 given in Appendix A there are weak orders that satisfy the same conditions. Let $>, \sim$ and $\succ^{\prime}, \sim$ be that kind of weak orders. Now, (iii) implies that we can choose $\gamma_{i j}$ such that

$$
\begin{aligned}
& 0<\gamma_{i j}<c_{i}\left(x_{1}^{j}, x_{2}\right)-c_{i}\left(x^{i}\right) \quad\left(i, j, k \in I: x_{2}^{k} \succsim^{i} x_{2}^{j} \text { and } x^{j}>x^{i}\right), \\
& \gamma_{i j}=0 \quad\left(i, j \in I: x^{j} \sim x^{i}\right) .
\end{aligned}
$$

Now, define

$$
z_{j}\left(x_{2}\right)=\min \left\{c_{i}\left(x_{1}^{j}, x_{2}\right)-c_{i}\left(x^{i}\right)-\gamma_{i j} \mid i \in I, x^{i} \preceq x^{j}\right\} \quad\left(j \in I, x_{2} \in X_{2}\right) .
$$

From the construction of $z_{j}$ and (iii), it follows that we have

$$
\begin{array}{ll}
z_{j}\left(x_{2}^{k}\right) \leq 0 \Rightarrow x_{2}^{k} \aleph^{k} x_{2}^{j} & (k, j \in I), \\
z_{j}\left(x_{2}^{k}\right)<0 \Rightarrow x_{2}^{k}<x_{2}^{j} & (k, j \in I) .
\end{array}
$$

Hence by Theorem 2.4 there is a function

$$
h\left(x_{2}\right)=\min _{i \in \mathbb{1}} h_{i}+\lambda_{i} z_{i}\left(x_{2}\right) \quad\left(x_{2} \in X_{2}\right)
$$

such that

$$
\lambda_{i}>0, h\left(x_{2}^{i}\right) \geq h_{i} \quad(t \in I)
$$

I will show that such a function $h$ satisfies the conditions of Theorem 4.6. Let $j \in I$ and $x_{2} \in X_{2}$, and suppose $h\left(x_{2}\right) \geq h\left(x_{2}^{j}\right)$. By the construction of $h$ we have then $h_{1}\left(x_{2}\right) \geq h_{j}$ and thus

$$
c_{i}\left(x_{1}^{j}, x_{2}\right)-c_{i}\left(x^{i}\right)-\gamma_{i j} \geq 0 \quad\left(i \in I: x^{j} \succeq x^{i}\right) .
$$

So one has

where

$$
\left(T_{h} c_{i}\right)\left(x^{j}\right) \geq c_{\mathrm{i}}\left(x^{i}\right)+\gamma_{i j} \quad\left(i, j \in I: x^{j} \geq x^{i}\right),
$$

$$
\left(T_{h} c_{i}\right)\left(x_{1}, x_{2}\right)=\inf _{h(w) \geq h\left(x_{2}\right)} c_{i}\left(x_{1}, v\right) \quad\left(i \in I, x \in X_{1} \times X_{2}\right)
$$


Hence, froni

$$
\begin{array}{ll}
\gamma_{i j}>0 & \left(i, j \in I: x^{j}-x^{i}\right), \\
\gamma_{i j}=0 & \left(i, j \in I: x^{j} \sim x^{i}\right),
\end{array}
$$

it follows that we have $\left(\boldsymbol{T}_{h} c_{i}\right)\left(x^{i}\right)=c_{i}\left(x^{i}\right)$ for all $8 \in 1$. So, the function $h$ satisfies the conditions of Theorem 4.6. Since $>\sim$ is a weak order, we have moreover

$$
\begin{array}{ll}
\left(\boldsymbol{T}_{h} c_{i}\right)\left(x^{j}\right) \leq\left(T_{h} c_{i}\right)\left(x^{i}\right) \Rightarrow x^{j}<x^{i} & (i, j \in I), \\
\left(T_{h} c_{i}\right)\left(x^{j}\right)<\left(T_{h} c_{i}\right)\left(x^{i}\right) \Rightarrow x^{j}<x^{i} & (i, j \in I),
\end{array}
$$

where we have used the property of a weak order $\succ, \sim$ that

$$
\text { not } x^{j} \succ x^{i} \Leftrightarrow x^{j}\left\langle x^{i} \quad(i, j \in I)\right. \text {. }
$$

Thus $D^{+}=\left\{\left(\boldsymbol{T}_{k} c_{i}, x^{i}\right)\right\}_{i \in I}$ satisfies the axiom of revealed preference, so that $\mathcal{F}_{d}\left(D^{\prime}\right) \neq \varnothing$ by Theorem 2.5. Hence, there is weakly separable function $T_{h} f=f \in \mathcal{F}_{d}(D)$ by Theorem 4.6 (b).

(b) (iii) $\Rightarrow$ (ii): Every function $T_{h} c_{i}$ in the above proof is weakly separable. Now, the assumption in (b) implies that its aggregator function $\bar{c}_{i}\left(x_{1}, \eta\right)$ is strictly increasing in $\eta$. In that case the constructed weakly separable function $g\left(x_{1}, h\left(x_{2}\right)\right) \in \mathcal{F}_{d}(D)$ in the proof of Theorem 4.6 (b) will also have these properties.

(c) One can show that the assumption made in (b) is satisfied by price and quantity data. Theorem 4.8 , which can be found at the end of this chapter, contains a complete proof to which I would like to refer the interested reader.

To prove (iii) $\Rightarrow$ (iv) we may construct functions $g$ and $h$ as in the proof of (b). These functions will have the mentioned properties, because they are derived from price and quantity data. Finally, the implication (iv) $\Rightarrow$ (ii) is trivial.

Condition (iii) in the above theorem is complicated. However, one may often simplify this condition. For empirical data one has in practice

$$
c_{i}\left(x_{1}^{j}, x_{2}^{k}\right) \neq c_{i}\left(x^{i}\right) \quad(i, j, k \in I: j \neq i \text { or } k \neq i) \text {. }
$$

So in practice we may simplify condition (iii) in Theorem 4.7 into:

(iii") There exist strict partial orders $\succ$ on $\left\{x^{i}\right\}_{i \in I}$ and $\succ^{\prime}$ on $\left\{x_{2}^{i}\right\}_{i \in I}$ such that

$$
c_{i}\left(x_{1}^{j}, x_{2}^{k}\right)<c_{i}\left(x^{i}\right) \Rightarrow x^{j}<x^{i} \text { or } x_{2}^{k}<x_{2}^{j} \quad(i, j, k \in I)
$$

(Note that for any preorder $\succ \sim$ it holds that $\succ$ is a strict partial order. More information concerning this sulbject may be lound in Appendix A.)

There is no simple test for the above condition. However, starting with a given strict partial order $\succ$, we cars rewrite the implication in condition (iii') as

$$
c_{i}\left(x_{1}^{j}, x_{2}^{k}\right)<c_{i}\left(x^{i}\right) \text { and not } x^{j} \prec x^{i} \Rightarrow x_{2}^{k}<^{\prime} x_{2}^{j} \quad\left(i, y_{1} k \in I\right) .
$$

This, however, is a problem of the revealed preference type for which a simple test is avallable. Assuming that $>$ is given, one may use Theorem A.5 in Appendix A, to test whether the corresponding strict partial order $>$ does exist. So, to test condition (iii), we may search for a suitable strict partial order $>$, such that there is a corresponding strict partial orcler $>$. I have used this approach in a computer program, which solves the problem quite fast.

Note that we cannot derive conserved properties from the conditions for weak 
separability. The reason for this is the fact that weak separability is not a property that can be derived from a given symmetry. Although we do know that a weakly separable utility function satisfies a certain symmetry, this symmetry jo uknown thll the subfunction is $h$ available.

The proof of the above theorem is quite complex. One may ask whether it woild be simpler to specialize it to linear prices. However, this is not the case. There is only one important difference. For linear prices we can prove that the functions $\tilde{c}_{i}\left(x_{1}, \eta\right)$ are strictly increasing in $\eta$. A general theorem that can be applicd to prove this is given in the next section.

\subsection{The Assumption of Strictly Increasing Cost Functions}

The application of Theorem 4.7 (b) is limited to consumer data sets for which the functions $\vec{c}_{i}\left(x_{1}, \eta\right)$, given in this theorem, are strictly increasing in $\eta$. Clearly they are increasing in $\eta$ and under certain conditions we can show that they are strictly increasing in $\eta$. In the following theorem such conditions are applied to a simplified case.

Theorem 4.8: Suppose the functions $h: X \rightarrow \mathbb{R}$ and $c: X \rightarrow \mathbb{R}$ are contunus, and the lower level sets

$$
\{x \in X \mid \alpha(x) \leq \alpha) \quad(\alpha \in \mathbb{R})
$$

compoct. Let the transformation $T_{h}$ be defined by

$$
\left(\boldsymbol{T}_{h} c\right)(x)=\inf _{h(v) \geq h(x)} c(v)
$$

Then:

(a) For tevery $x \in X$ there exists a $v \in X$ such that $\left(T_{h} c\right)(x)=c(v)$ and $h(v) \geq h(x)$.

(b) If $-c$ is locally nonsatiated on $\left\{x \in X \mid h(x)>\eta_{0}\right\}$, where

$$
\eta_{0}=\inf _{x \in X} h(x),
$$

then the function

$$
\bar{c}(\eta)=\operatorname{lnf}_{h(v) \geq \eta} c(v)
$$

is stictictly increasing on $h(X)$.

Proof (a): Let $x \in X, \varepsilon>0$ and put

$$
S=\{v \in X \mid h(v) \geq h(x)\} \cap\left\{v \in X \mid c(v) \leq\left(T_{h} c\right)(x)+\varepsilon\right\} .
$$

Then the definition of $\boldsymbol{T}_{h} c$ implies that one has

$$
\left(T_{k} c\right)(x)=\inf _{v i \in S} c(v)
$$

Hence, because $c$ is continuous and $S$ compact, there is a. $y \in S$ such that $\left(T_{h} c\right)(x)=c(v)$.

(b): Let $\left.\eta_{1}, \eta_{2} \in h_{(X}\right)$ and suppose $\eta_{2}>\eta_{1}$. By (a) there is an $y \in X$ such that $\bar{c}\left(\eta_{2}\right)=c(v)$ and $h(v) \geq \eta_{2}$. Now, simce $h$ is continuous and $h(v) \geq \eta_{2}>\eta_{1}$, there is a neighbourhood $V$ of $v$ such that $h(x)>\eta_{1}$, for every $x \in V$. Furthermore, if $-c$ is locally nonsatiated at $v$, way pick $x \in V$ such that $c(x)<c(v)$ and we have thus

$$
c\left(\eta_{1}\right) \leq c(x)<c(v)=\bar{c}\left(\eta_{2}\right)
$$




\section{SOME NONPARAMETRIC THEORY}

\subsection{Introduction}

In the previous chapters I suggested an approach to consumer theory from an empirical point of view. This approach departs from a given empirical data set - instead of given preferences - and opens a promising field of research with many empirical applications. This chapter, however, contains a taste of a theoretical research, which is not directly connected with empirical applications.

The basis of the previous chapters was symmetry. The present chapter considers the following other interesting theoretical problems:

1. What are the properties of the rationalizing set of preonclers or functions for a given data set?

2. What can we say about convergence of such functions?

3. Is there a way to derive a utility function from the axiom of revealed preference theorem for an infinite data set?

4. Which conditions do we need to derive rational demand from cost minimizing behaviour or from utility maximizing behaviour?

I will try to give an answer to such questions. The theory, discussed in this chapter, is suitable for application do data sets of an infinite number of elements.

\subsection{Closed Mappings}

This section considers transformations that map a rationalizing set of preference orders or utility functions onto itself. The next theorem contains an example that applies to preference ordiers.

Theorem 5.1: Suppose $R_{0} \subset R_{u}(D)$ and let the preorder $\succ$, be defined as the intersection of $\mathcal{R}_{0,}$ i.e. the preorder that satisfies

$$
x \succsim y \Leftrightarrow x \succsim^{\prime} y \text { for all } \succsim^{\prime} \in R_{0} .
$$

Then this preorder is element of $R_{u}(D)$.

Proof: Left to the reader.

To describe the general idea we need the following definition,

Definition: Let $S$ be a set of elements. Then $S$ is said to be closed with respect to a given transformation $T_{3}$ if $T^{*}$ maps all elements of $S$ on $S$. Similarly, we say that $S$ is closed with respect to $T$, if $\boldsymbol{T}$ is transformation that maps all subsets of $S$, or all sequences in $S$, on $S$.

For example, the set $[0,1] \subset \mathrm{C}$ is closed with respect to the infimum operation; because the infimum of a subset of $[0,1]$ is element of $[0,1]$.

Now, we may translate Theorem 5.1 into a more compact version that is also valid for $R_{c}(D)$.

Theorem 5.2: For every data set $D$, the families $R_{w}(D)$ and $\mathcal{R}_{c}(D)$ are closed woth respect to the intersection of collections preorders.

Families of functions $\mathcal{F}_{d}(D)$ may also be closed with respect to certain transformations, and it seems fruitful to pay further attention to the implications of this property. Below are some examples of such 
transiomations.

Theorem 5.3: Every family $\mathcal{F}_{a}(D)$ and $\mathcal{F}_{d}(D)$ is closed with respect to the following transformations of $\left\{f_{i}\right\}_{i \in I}$ to a function $f$ :

(a) A monotonic transformation $f=$ mo $f_{1}$, where in is a strictly increasing function.

(b) $f=\min _{i} f_{i}$ and $f=\max _{i} f_{i}$.

(c) $f=\sum_{i=1}^{n} \alpha_{i} f_{i} \quad\left(\alpha_{i}>0\right)$

(d) $f=\prod_{i=1}^{7} f_{i}^{\alpha_{i}} \quad\left(\alpha_{i}>0, f_{i} \geq 0\right)$

Proof: This follows directly from Theorem 5.4 given below.

Below follows a theorem that treats the common property of the uransformations in the above theorem.

Theorem 5.4: Suppose $T^{T}$ is a transformation, which maps collections of functions $\left\{f_{i}: X \rightarrow \mathbb{R}\right\}_{\text {i }}$ on an image function $f: X \rightarrow \mathbb{R}$. If $\boldsymbol{T}$ has for every map of $\left\{f_{i}\right\}_{i \in I}$ on $f$ the property

$$
f_{i}(x) \leq f_{i}(y) \forall i \in I \Rightarrow f(x) \leq f(y) \quad(x, y \in X),
$$

then any family $\mathcal{F}_{u}(D)$ is closed with respect to $\boldsymbol{T}$. Similarly, if $\boldsymbol{T}$ thas the property

$$
f_{i}(x)<f_{i}(y) \forall i \in I \Rightarrow f(x)<f(y) \quad(x, y \in X)
$$

then any family $\mathcal{F}_{c}(D)$ is closed with respect to $\boldsymbol{T}$.

Proof: Obvious.

For utility maximizing behaviour one has the following theorem; which has no counterpart for cost minimizing behaviour:

Theorem 5.5: Every family $\mathcal{F}_{u}(D)$ is closed with respect to the pointwise infimum and supremum over collections of functions, such that the limit function is a well-defined finite function.

Proof: For any set $\left\{a_{i}\right\}$ and $\left\{b_{i}\right\}$ one has

$$
a_{i} \leq b_{i} \forall i \Rightarrow \sup _{i} a_{i} \leq \sup _{i} b_{i} \text { and } \inf _{i} a_{i} \leq \inf _{i} b_{i} \text {. }
$$

Thus we may apply Theorem 5.4.

A direct proof for the infimum operation is as follows. We have for $\left(f_{i}\right) \subset \mathcal{F}_{u}(D)$ and arbitrary $\left(c, x^{\prime \prime}\right) \in D$ the implication

$$
c(x) \leq c\left(x^{\prime}\right) \Rightarrow f_{i}(x) \leq f_{i}\left(x^{\prime}\right) \forall i \Rightarrow \inf _{i} f_{i}(x) \leq \inf _{i} f_{i}\left(x^{\prime}\right) \text {. }
$$

Hence, for the function

$$
f=\inf _{i} f_{i}
$$

we have $f \in \mathcal{F}_{w}(D)$. A similar result holds for the supremum operation.

There is no analogous theorem for $\mathcal{F}_{c}(D)$. However, there is a similar theorem valid for finite index sets $I$, or more general, for attained maxma and minima. This is stated in Theorem $5.3(b)$.

Remark: An illustration of the constuction of a counter example, such that one has $\left\{f_{i}\right\} \subset \mathcal{F}_{c}(D)$ and the function

$$
f=\inf _{i} f_{i}
$$

is not contained in $\mathcal{F}_{e}(D)$, is the following. Suppose $h: X \rightarrow \mathbb{R}_{++} \in \mathcal{F}_{c}(D)$, 
$x^{\prime}, x^{\prime \prime} \in X^{\prime \prime},\left(c, x^{\circ}\right) \in D$ and $c\left(x^{21}\right)<c\left(x^{\prime}\right)$. Put $f=h / s$ for all $\& \in \mathbb{N}$. In that case one has $f_{i} \in \mathcal{F}_{d}(D)$ for all $i$ and $f=0$. If $f \in \mathcal{F}_{c}(D)$ then we must have $f\left(x^{* i}\right)<f\left(x^{*}\right)$; since $c\left(x^{\prime \prime}\right)<c\left(x^{\prime}\right)$. However, this is not the case, because $f=0$. So, we have $f \notin \mathcal{F}_{c}(D)$.

Why is there no analogous theorem for $\mathcal{F}_{d}(D)$ ? As we have seen in the counter example in the remark above, there may occur satiation when the limit is taken. In case of utility maximization this is no problem, any constant utility function is a rationalizing function, but conoerning cost minimization we have the following problem. Suppose $\left(f_{i}\right) \subset \mathcal{F}_{d}(D)$ and $\left(c, x^{\prime}\right) \in D$. Now, in order to derive an analogous theorem for cost mimimization, we need the implications

$$
c(x)<c\left(x^{*}\right) \Rightarrow f_{i}(x)<f_{i}\left(x^{*}\right) \forall i \Rightarrow \inf _{i} f_{i}(x)<\inf _{i} f_{i}\left(x^{\prime}\right)
$$

However, the latter implication is not valid in general. As we have seen in the remark above, one may have $f_{i}(x)<f_{i}\left(x^{\prime}\right)$ for all and

$$
\inf _{i} f_{i}(x)=\inf _{i} f_{i}\left(x^{\prime}\right) \text {. }
$$

For the supremum over functions we have the same problem.

\subsection{Approximately Finite Data}

An important problem in the revealed preference theory is the specification of conditions, which have to be satisfied by a given consumer data set, such that there exists a rationalizing utility function. When the data set has a finite number of elements then the axiom of revealed preference is a necessary and sufficient condition. However, this is not the case for data sets that contain an infinite number of elements, for instance, a data set described by using a continuous index parameter $i \in I$. Then we need additional restrictions on the data besides the axiom of revealed preference. In this section I give an example of such restrictions.

Definition: When we have

$$
S \in \cup_{j} B_{j}
$$

then $\left\{B_{j}\right\}$ is called a cover of $S$.

Using the above definition, we may now formulate the central definition in this section.

Definition: Suppose $D=\left\{\left(c_{i}, x^{i}\right)\right\}_{i \in}$ is a data set of consumen behaviour, $\phi_{e}$ is an efficiency transformation, and $e \in[0,1]$. Let us use the notation

$$
\Phi_{\alpha} D=\left\{\left(c_{i}^{\alpha}, x^{i}\right)\right\}_{i \in K}
$$

If for every $a \in[0, e)$ there is a finite subset $J \subset I$, a cover $\left\{B_{j}\right\}_{j \in d}$ of $\left\{x_{i \in I}\right.$, and strictly increasing monotonic transformations $m_{i}$, $i \in I$, that satisfy

$$
m_{k} \circ c_{k}^{a}\left(x^{i}\right)-m_{k} \circ c_{k}\left(x^{k}\right) \geq c_{l}^{c}\left(x^{j}\right)-c_{l}\left(x^{l}\right) \quad\left(j, l \in J, \quad \dot{x}, k \in I: x^{i} \in B_{j}, \quad x^{k} \in B_{l}\right)
$$

then $D$ is said to be approximately finite at efficiency level $c$

The idea behind this definition is that one may apply theoretical results, concerning finite data sets, to approximately finite data sets. This is done in the following theorem, where a rationalizing utility function is derived for an infinite data set.

Theorem 5.6: Suppose $D=\left\{\left(c_{i}, x^{i}\right)\right\}_{i \in I}$ is a data set of conswimer behaviour, $\phi_{e}$ is an efficiency transformation. If $\Phi_{e} D$ satisfies the axiom of reveded 
preference and $D$ is approximately finite at efficiency level $e$ then

$$
F_{d}\left(\Phi_{a} D\right) \neq 0 \quad(a \in(0, \mathbb{E})) \text {. }
$$

Proof: Let $a \in[0, e]$ and let us use the notation

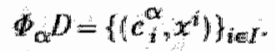

Suppose $D$ is approximately finte at efficiency level $e$. Then we may choose a fintte subset $J \subset I$, a cover $\left\{B_{j}\right\}_{j \in J}$ of $\left\{x^{i}\right\}_{i \in I}$ and strictly increasing monotonic transformations $m_{i}$ that satisfy

$$
m_{k} \circ c_{k}^{a}\left(x^{i}\right)-m_{k} c_{k}\left(x^{k}\right) \geq c_{l}^{e}\left(x^{j}\right)-c_{l}\left(x^{l}\right) \quad\left(j, l \in J_{,}, k, k \in I: x^{i} \in B_{j}, x^{k} \in B_{i}\right)
$$

The data $\Phi_{e} D$ satisfy the axiom of revealed preference, hence this is also the case for any finite data subset

$$
D^{\prime}=\left\{\left(c_{j}^{e}, x^{j}\right)\right\}_{j \in j} \subset \Phi_{e} D
$$

Hence, as shown in the proof of Theorem 2.5 , there is a function $g \in \mathcal{F}_{d}(D)$ of the form

$$
g(x)=\min _{j \in J} g_{j}+\lambda_{j}\left[c_{j}^{c}(x)-c_{j}\left(x^{j}\right)\right] \quad(x \in X),
$$

where $\lambda_{j}>0$ and $g\left(x^{j}\right) \geq g_{j}$ for all $j \in J$. Now, define

$$
\begin{aligned}
& I_{j}=\left\{i \Subset I \mid x^{i} \in B_{j}\right\}, \\
& f(x)=\min _{j \in J} g_{j}+\lambda_{j} \inf _{i \in I_{j}}\left[m_{i} \circ C_{i}^{a}(x)-m_{i} \circ c_{i}\left(x^{i}\right)\right] \quad(x \in X) .
\end{aligned}
$$

To show that $f \in \mathcal{F}_{d}\left(\Phi_{a} D\right)$, let $i \in I$ be arbitrary. Since $\left\{B_{j}\right\}$ is a cover of $\left\{x^{i}\right\}$, we may pick $j \in J$ such that $x^{i} \in B_{j}$ and thus $i \in I_{j}$. Then we have

$$
\begin{array}{lll}
c_{i}^{a}(x) \leq c_{i}\left(x^{i}\right) \Rightarrow m_{i} \circ c_{i}^{a}(x) \leq m_{j} \circ c_{i}\left(x^{i}\right) \Rightarrow f(x) \leq g_{j} & (x \in X), \\
c_{i}^{a}(x)<c_{i}\left(x^{i}\right) \Rightarrow m_{i} \circ c_{i}^{a}(x) \leq m_{i} \circ c_{i}\left(x^{i}\right) \Rightarrow f(x)<g_{j} \quad(x \in X) . &
\end{array}
$$

So $f \in \mathcal{F}_{d}\left(\Phi_{a} D\right)$ provided we can show that one has $g_{j} \leq f\left(x^{i}\right)$. From

$$
\begin{aligned}
& m_{k} \circ c_{k}^{a}\left(x^{i}\right)-m_{k} \circ c_{k}\left(x^{k}\right) \geq c_{l}^{i}\left(x^{j}\right)-c_{l}\left(x^{l}\right) \quad\left(j, l \in \mathcal{J}, i, k \in I: x^{i} \in B_{j}, x^{k} \in B_{l}\right), \\
& g\left(x^{j}\right) \geq g_{j}
\end{aligned}
$$

it follows that we have indeed

$$
\begin{aligned}
& f\left(x^{i}\right)=\min _{l \in J} g_{l}+\lambda_{l} \inf _{k \in I_{l}}\left[m_{k} \circ c_{k}^{a l}\left(x^{i}\right)-m_{k} \circ c_{k}\left(x^{k}\right)\right] \geq \\
& g\left(x^{j}\right)=\min _{l \in J} g_{l}+\lambda_{l}\left[c_{l}^{l}\left(x^{j}\right)-c_{l}\left(x^{l}\right)\right] \geq g_{j} .
\end{aligned}
$$

One may also apply the proof of the above theorem to derive utility functions that are concave, monotonically increasing or upper semicontinuous. Therefore we only need the assumption that the cost functions have the same property. This result follows directly from the properties of the utility function, which is constructed in the proof of "Theorem 5.6. We can derive these properties as follows. The pointwise infimum over concave functions is concave, see Rockafellar (1970, Theorem 5.6). For monotonjcally increasing cost furictions the resulting property is obvious. Furthermore, the pointwise infimum over upper semicontinuous functions is upper semicontinuous, because the intersection of closed sets is a closed set.

Definition: A function $f: X \rightarrow \mathbb{R}$ is said to be upper semicontinuous if all its upper llevel sets

$$
\{x \in X \mid f(x) \geq \alpha\} \quad(\alpha \in \mathbb{R})
$$

are closed. Similarly, a function $f: X \rightarrow \mathbb{R}$ is said to be lower semicontinuous if all its lower level sets 


$$
\{x \in X \mid f(x) \leq \alpha\} \quad(\alpha \in \mathbb{R})
$$

are closed.

\subsection{Examples of Approximately Finite Data}

For the analysis of infinite data sets it is useful to have conditions available which imply that the data set is approximately finite. The following theorem provides an example of approximately finite data.

Theorem 5.7: Let $X$ be a metric space and $K \subset X$ compact. Suppose $P: K \times X \rightarrow \mathbb{R}_{+}$is continuous on $K \times K$ and $P(x, x)=1$ for all $x \in K$. Then for the efficiency transformation $\Phi_{e}$ an Example 2.3 , any data set $D=\left\{\left(c_{i}, x^{i}\right)\right\}_{i \in I}$ of the form

$$
\left(c_{i}, x^{i}\right)=\left(P\left(x^{i}, \cdot\right), x^{i}\right) \quad(i \in I)
$$

is approximately finite at every efficiency level $e$.

Proof: Let us use the notation

$$
\Phi_{\alpha} D=\left\{\left(c_{i}^{\alpha}, x^{i}\right)\right\}_{i \in I} .
$$

and let $a \in[0, e)$ and $\varepsilon=e / a-1$. Because $P$ is uniformly continuous on the conapact set $K \times K$, we can choose $\delta>0$ such that

$$
\left\|x^{k}-x^{l}\right\|+\left\|x^{i}-x^{j}\right\| \leq \delta \Rightarrow\left\|P\left(x^{k}, x^{i}\right)-P\left(x^{l}, x^{j}\right)\right\| \leq \varepsilon \quad(i, j, k, l \in I) .
$$

Since $K$ is compact, there is a finite finite cover of $K$ consisting of open balls of diameter $\delta / 2$. Hence, there is a finite subset $J C I$ and a cover $\left\{B_{j}\right\}_{j \in J}$ of $\left\{x^{i}\right\}_{i \in I}$, such that the diameter of $B_{j}$ is less or equal to $\delta / 2$ and

$$
\begin{aligned}
& x_{j} \in B_{j} \quad(j \in J), \\
& B_{j} \cap B_{l}=\varnothing \quad(j, l \in J: j \neq l) .
\end{aligned}
$$

Now, put

$$
m(z)=e^{-1} a z \quad(z \in \mathbb{R}) .
$$

Pick arbitrary $j, l \in J$ and $i, k \in I$. If $x^{k}=x^{i}$ then it follows that we have

$$
\begin{aligned}
& x^{k} \in B_{l}, x^{i}=x^{k} \in B_{j} \Rightarrow l=j \Rightarrow \\
& \operatorname{moc}_{k}^{a}\left(x^{i}\right)-\operatorname{moc}_{k}\left(x^{k}\right)=m(1)-m(1)=1-1=c_{l}^{e}\left(x^{j}\right)-c_{l}\left(x^{l}\right) .
\end{aligned}
$$

At the other hand, in case $x^{k} \neq x^{i}$, one has

$$
\begin{aligned}
& x^{k} \in B_{l}, x^{i} \in B_{j} \Rightarrow\left\|x^{k}-x^{l}\right\|+\left\|x^{i}-x^{j}\right\| \leq \delta \Rightarrow P\left(x^{k}, x^{i}\right) \geq P\left(x^{l}, x^{j}\right)-\varepsilon \Rightarrow \\
& m \circ c_{k}^{a}\left(x^{i}\right)-m \circ c_{k}\left(x^{k}\right)=m\left(a^{-1} P\left(x^{k}, x^{i}\right)\right)-m(1) \geq \\
& m\left(a^{-1} P\left(x^{l}, x^{j}\right)-\varepsilon\right)-m(1)=e^{-1} P\left(x^{j}, x^{j}\right)-1 \geq c_{i}^{e}\left(x^{j}\right)-c_{l}\left(x^{j}\right) .
\end{aligned}
$$

Hence $D$ is approximately finite al efficiency level $e$.

Note that the continuity assumption in Theorem 5.7 can be weakened, because one may replace

$$
\left|P\left(x^{k}, x^{i}\right)-P\left(x^{i}, x^{j}\right)\right| \leq \varepsilon
$$

in the proof of Theorem 5.7, by the weaker inequality

$$
P\left(x^{k}, x^{i}\right)-P\left(x^{l}, x^{j}\right) \leq \varepsilon
$$

without altering the result of the proor. This means that, by a more specific choice of $\left\langle B_{j}\right\}_{j \in d}$, one may also prove a theorem which allows for certain discontinuities in $P$.

Now let us consider price and quantity data described by $(p(x), x)$, where $p$ maps quantity data $x$ on price data. The following example tells us when we may scale the prices $p$ such that the resulting price and quantity data is 
approximately finite.

Theorem 5.8. Suppose $K \subset \mathbb{R}_{+}^{n}$ is a closed bounded set and $p: K \rightarrow \mathbb{R}_{++}^{n}$. Suppose the Laspeyres price index $P\left(x^{*}, x\right)=p\left(x^{\prime}\right) x / p\left(x^{n}\right) x^{*}$ is continuous on $K \times K$. If conswmers choices are restricted to $K$ then any price and quantity data set $D=\left\{\left(p^{i}, x^{i}\right)\right\}_{\text {iet }}$, where

$$
p^{i}=\left[p\left(x^{i}\right) x^{i}\right]^{-1} p\left(x^{i}\right) \quad(i \in I)
$$

is approximately finite at every efficiency level $e$.

Proof: We may apply Theorem $5 . \pi$, becaluse any closed bounded subset of $R^{m}$ is compact.

\subsection{Utility Maximization and Cost Minimization}

The question under which conditions is a utility maximizing consumer a cost minimizer and vice versa is an interesting one. In this section I will give two wheorems that are concerned with such conditions. The first theorem states that a utility maximizing consumer with a locally nonsatiated utility function is a cost minimizer for upper semicontinuous cost functions.

Theorem 5.9: Let $\left(c, x^{\prime}\right) \in \mathcal{D}_{u}(f)$. Suppose $f: X \rightarrow \mathbb{R}$ is locally nonsatiated on $X^{\prime}$ and c is upper semicontinuous on $X^{*}$, where

$$
X^{x}=\left\{x \in X \mid f(x)=f\left(x^{*}\right)\right\} \cap\left\{x \in X \mid c(x)<c\left(x^{x}\right)\right\} .
$$

Then $\left(c, x^{\prime}\right) \in \mathcal{D}_{c}(f)$.

Proof: Suppose $c(x)<c\left(x^{\prime}\right)$ for an arbitrary $x \in X$. Then $\left(c, x^{\prime}\right) \in \mathcal{D}_{u}(f)$ implies $f\left(x^{\prime}\right) \geq f(x)$. We have to show that $f(x)=f\left(x^{\prime}\right)$ is impossible. Therefore suppose $f(x)=f\left(x^{\prime}\right)$. Since $c$ is upper semicontinuous at $x$ by assumption, there is a neighbourhood $V \subset X$ of $x$ such that $c(v)<c\left(x^{\prime}\right)$ for all $v \in V$. Because $f$ is locally nonsatiated at $x$, there is a $v \in V$ such that $f(v)>f(x)$. Furthermore, $\left(c, x^{*}\right) \in D_{u}(f)$ and $c(v)<c\left(x^{\prime}\right)$ implies $f\left(x^{*}\right) \geq f(v)$. Thus $f\left(x^{\prime}\right)>f(x)$, which contradicts $f(x)=f\left(x^{\prime}\right)$.

The dual of the above theorem shows conditions under which a cost minimizer is a utility maximizer.

Theorem 5.10: Let $\left(c, x^{\prime}\right) \in \mathcal{D}_{c}(f)$. Suppose $f: X \rightarrow \mathbb{R}$ is tower semicontinuous on $X^{*}$, and $-c$ is locally nonsatiated on $X^{*}$, where

$$
X^{\prime \prime}=\left\{x \in X \mid f(x)>f\left(x^{\prime}\right)\right\} \cap\left\{x \in X \mid c(x)=c\left(x^{\prime}\right)\right\} .
$$

Then $\left(c, x^{*}\right) \in D_{u t}(f)$.

Proof: Similar to the proof of Theorem 5.9.

One may apply the above theorems as follows to price and quantity data.

Theorem 5.11: Stupose $f: \mathbb{R}_{+}^{n} \rightarrow \mathbb{R}$ is a locally nonsatiated function and let price and quantity $(p, x) \in \mathcal{D}_{u}(f)$. Then $\left(p, x^{\prime}\right) \in \mathcal{D}_{c}(f)$.

Proof: The function $c(x)=p x$ is upper senicontinuous, hence we can apply Theorem 5.9 .

Theorem 5.12: Suppose $f: \mathbb{R}_{+}^{n} \rightarrow \mathbb{R}$ is lower semicontinuous and let price and quartity $\left(p, x^{\prime}\right) \in \mathcal{D}_{d}(f)$. Then $\left(p, x^{\prime}\right) \in \mathcal{D}_{u}(f)$.

Proor: The function $-c(x)=-p x$ with domain $\mathbb{R}_{+}^{m}$ is locally nonsatiated at every $x \neq 0$ in $\mathbb{R}_{*}^{n}$. Furthermore 0 is not contained in

$$
X^{n}=\left\{x \in X \| f(x)>f\left(x^{\prime}\right)\right\} \cap\left\{x \in X \mid c(x)=c\left(x^{\prime}\right)\right\},
$$

because $f(0)>f\left(x^{\prime}\right)$ implies $x^{\prime} \neq 0$, thus $p 0 \neq p x^{\prime}$. Hence, the result follows from Theorem 5.10 . 


\subsection{Utility Functions and Convergence}

When is a sequence of functions $f_{n} \in J_{d}(D)$, neN, converging pointwise to a limit function in $\mathcal{F}_{d}(D)$ ? Also interesting is the following question for an efficiency transformation $\Phi_{e}$. When is a sequence of functions

$$
f_{n} \in \mathcal{F}_{d}\left(\Phi_{e_{n}} D\right), e_{m} * e_{,}
$$

pointwise converging to a limit function in $\mathcal{F}_{d}\left(\Phi_{e} D\right)$ ?

The following theorem shows that the efficiency levels, which are consistent: with cost minimizing behaviour, form a closed set.

Theorem 5.13: Suppose $D$ is a data set, $\Phi_{e}$ is an efficiency transformation, and $e \in[0,1]$. Suppose for all

$$
\left(c^{a}, x^{*}\right) \in \Phi_{a} D \quad(a \in[0, e))
$$

that $c^{a}(x)$ is continuous with respect to a, for every fixed $x \in X$. Then

$$
f \in \mathcal{F}_{c}\left(\Phi_{a} D\right) \text { for au } a \in[0, e) \Leftrightarrow f \in \mathcal{F}_{c}\left(\Phi_{e} D\right) \text {. }
$$

Proof: $(\Leftarrow)$ This follows from Theorem 2.1 .

$(\Rightarrow)$ : Suppose $f \in \mathcal{F}_{c}\left(\Phi_{a} D\right)$ for all $a \in[0, e)$. Let $\left(c^{e}, x^{3}\right) \in \Phi_{e} D, x \in X$, and suppose $f(x) \geq f\left(x^{\prime}\right)$. Then one has

$$
c^{a}(x) \geq c^{a t}\left(x^{\prime}\right)=c^{e}\left(x^{\prime}\right) \quad(a \in[0, e))
$$

and the continuity assumption implies thus

$$
c^{e}(x)=\lim _{a \rightarrow e} c^{a}\left(x^{\prime}\right) \geq c^{e}\left(x^{n}\right)
$$

Hence, $f \in \mathcal{F}_{c}\left(\Phi_{e} D\right)$.

The above theorem has no counterpart for utility maximizing behaviour, and the efficiency levels, which are consistent with utility maximizing behaviour, may form an open set. For utility maximizing behaviour one has in turn the following theorem, which has no counterpart for cost minimizing behaviour.

Theorem 5.14: Every family $\mathcal{F}_{u}(D)$ is closed with respect to the pointwise $\lim \inf f_{n}$ and $\limsup f_{n}$ for sequences of functions $f_{n} n \in \mathbb{N}_{\text {; }}$ such that the limit function is a well-defined finite function.

Proof: We may apply Theorem 5.4 as in the proof for Theorem 5.5, concerning: the pointwise infimum or supremum over rationalizing functions.

Using the above theorem we can derive a theorem for converging efficiency levels.

Theorem 5.15: Suppose $D$ is a data set and $\Phi_{e}$ is an efficiency transformation. Suppose there is a sequence of functions

$$
f_{n} \in \mathcal{F}_{u}\left(\Phi_{e_{n i}} D\right) \quad(n \in \mathbb{N})
$$

where $e_{n}$ te, such that lim inf $f_{n}$, or limsup $f_{n}$ converges pointwise to $f: X \rightarrow \mathbb{R}$. Then one has

$$
f \in \mathcal{F}_{u}\left(\Phi_{a t} D\right) \quad(a \in[0, e)) .
$$

Proof: We prove the theorem for lim inf $f_{\pi}$. The proof for limsup $f_{n i}$ is analogous. From Theorem 2.1 follows

$$
f_{n} \in \mathcal{F}_{u}\left(\Phi_{e_{n}} D\right) \quad(n \geq m, m \in \mathbb{N})_{s}
$$

since $f_{n} \in \mathcal{F}_{u}\left(\Phi_{e_{n}} D\right)$ and $e_{n}$ is increasing. Hence from Theorem 5.14 it follows that 


$$
\lim _{n \rightarrow \infty} \text { inf } f_{n} \in \mathcal{F}_{u}\left(\Phi_{e_{m}} D\right) \quad(m \in \mathbb{N}) \text {. }
$$

Thus the result for $a \in[0, e)$ follows from Theorem 2.1 .

The following theorem shows when a sequence of functions

$$
f_{n} \in \mathcal{F}_{u}\left(\mathscr{P}_{e_{n}} D\right), e_{n} t e_{\text {, }}
$$

converges pointwise to a limit function in $\mathcal{F}_{d}\left(\Phi_{e} D\right)$.

Theorem 5.16: Suppose $D$ is a data set and $\Phi_{*}$ is an efficiency transformation. Suppose there is a sequence of functions.

$$
f_{\mathrm{n}} \in \mathcal{F}_{\mathrm{u}}\left(\Phi_{\mathrm{e}_{n}} D\right) \quad(n \in \mathbb{N})_{*}
$$

where $e_{n}+e$, such that $\lim \inf f_{m}$, or $\lim \sup _{n}$ converges pointwise to $f: X \rightarrow \mathbb{R}$. Suppose for all

$$
\left(a^{a}, x^{\prime \prime}\right) \in \Phi_{a} D \quad(a \in[0, e))
$$

that $c^{a}(x)$ is continuons with respect to $a$, for every fixed $x \in X$. Then one has $f \in \mathcal{F}_{d}\left(\Phi_{Q} D\right)$, if the following conditions are satisfied:

(i) For every $\left(c, x^{\prime}\right) \in \Phi_{a} D$, a $[0, e)$, one has $f$ locally nonsatiated, and $c$ upper semicontinuous on

$$
\left\{x \in X^{\prime} \mid f(x)=f(x)\right) \cap\left\{x \in X \mid c(x)<c\left(x^{2}\right)\right\}
$$

(ii) For every $\left(c, x^{\prime}\right) \in \Phi_{\mathrm{e}} D$, one has $f$ lower semicontinuous, and $-c$ locally nonsatiated, on

$$
\left\{x \in X \mid f(x)>f\left(x^{\prime \prime}\right)\right\} \cap\left\{x \in X \mid c(x)=c\left(x^{\prime \prime}\right)\right\} .
$$

Proor: Since $f_{n} \in \mathcal{F}_{u l}\left(\Phi_{e_{n}} D\right)$ converges to the limit function $f$ and $e_{n} \star \varepsilon$, one has

$$
f \in \mathcal{F}_{a}\left(\Phi_{a} D\right) \quad(a \in[0, e))
$$

by Theorem 5.15. Thus by Theorem 5.9 and condition (i) one has

$$
f \in \mathcal{F}_{c}\left(\Phi_{a} D\right) \quad(a \in[0, e)) \text {. }
$$

Hence $f \in \mathcal{F}_{c}\left(\Phi_{c} D\right)$ by Theorem 5.13. Finally, Theorem 5.10 together with condition (ii), imply $f \in \mathcal{T}_{d}\left(\Phi_{e} D\right)$.

\subsection{Existence of a Rationalizing Concave Function}

Using the nonparametric theory we can give conditions for price and quantity data, such that the existence of a rationalizing concave famction for efficincy fever $a \in[0, e)$ implies the existence of a rationalizing concave function for efficiency level $e$.

Theorem 5.17: Suppose $D$ is a set of price and quantity data and $\Phi_{e}$ is the officiency transfomation given in Example 2.3. Suppose there is a seguence of concave functions $f_{n}: \mathbb{R}^{k} \rightarrow \mathbb{R}, n \in \mathbb{N}$, such that

$$
f_{n} \mid \mathbb{R}_{+}^{k} \in \mathcal{F}_{d}\left(\Phi_{c_{n}} D\right), c_{n}+e .
$$

Then there exists a continuons locally nonsatiated concave function $f: \mathbb{R}_{+}^{k} \rightarrow \mathbb{R}$ in $F\left(\Phi_{1} D\right)$ of the following conditions are satisfied:

(i) The sequence $f_{n}, n \in \mathbb{N}$, is pointwise bounded.

(ii) There are $x^{0} \in \mathbb{R}_{+}^{k}$ and $K>0$ such that

$$
f_{n}\left(\lambda x^{(j)}\right) \geq \lambda K \quad(\lambda>0, n \in \mathbb{N}) \text {. }
$$

Proof: Because $\mathbb{R}_{+}^{k}$ is a closed subset of $\mathbb{R}^{k}$, there is a subsequence of $f_{m} \mid \mathbb{R}^{k}$, $m \in \mathbb{N}$, converging to a continuous concave function $f: \mathbb{R}_{+}^{k} \rightarrow \mathbb{R}$ (See Rockafellar, 1970, Theorem 10.9). Now,

$$
f_{n}\left(\lambda x^{0}\right) \geq \lambda k \quad(n \in \mathbb{N})
$$


implies $f\left(\lambda x^{0}\right) \geq \lambda K$. Thus for every $x \in \mathbb{R}_{*}^{k}$, there is $\lambda>0$ such that $f\left(A x^{0}\right)>f(x)$. Hence, since $f$ is concave, it is easy to show that $f$ is locally nonsaliated: Furthermore, $f$ is lower semicontinuous, since $f$ is continuous. So $f$ is a locally nonsatiated lower semicontinuous limit function, thus $f \in \mathcal{F} d(\Phi, D)$ by Theorem 5.15. Both conditions (i) and (ii) in this theorem are satisfied for price and quantity data $D$ and the given efficiency transformation $\Phi_{e}$ : condition (i), because the cost functions in $\Phi_{a} D, a \in[0, e$, are upper semicontinuous; condition (ii), because for the cost functions $c$ in $\Phi_{e} D$ one has $-c$ locally nonsatiated.

The above theorem may be useful in further research of the following idea. Let us start with the assumption that the consumer data $\phi_{e} D$ satisfy the axiom of revealed preference. Our goal is the derivation of a concave utility function $f \in \mathcal{F}\left(\Phi_{e} D\right)$. A way to derive such a function might be as follows.

Stupose $D$ is approximately finite at efficiency level e. Then by Theorem 5.6 there is a sequence of functions

$$
f_{n} \in \mathcal{F}_{d}\left(\Phi_{e_{n}} D\right), e_{n 2} * \text { e. }
$$

Moreover, if we can show that the functions $m_{j} \circ c_{i}^{e_{m}}, i \in I$, in the proof of Theorem 5.6 can be extended to finite concave functions on $\mathbb{R}^{k}$, there is a sequence of concave functions $f_{n}: \mathbb{R}^{k} \rightarrow \mathbb{R}, n \in \mathbb{N}$, such that

$$
f_{n} \mid \mathbb{R}_{+}^{k} \in \mathcal{F}_{d}\left(\Phi_{e_{n}} D\right), e_{n} * \text {. }
$$

Then, finally, we may use Theorem 5.17 to derive the existence of a concave utility function $f \in \mathcal{F}\left(\Phi_{c} D\right)$, prowided we can show that the derived sequence $f_{m}$, $n \in \mathbb{N}$, satisfies conditions (i) and (ii) in this theorem.

Thus we end this chapter with an open problem: it remains to specify neat conditions that allow us to elaborate the above outline of a proof. 


\section{NONPARAMETRIC ANALYSIS OF AGGREGATE DATA}

\subsection{Introduction}

Empirical studies of consumer data, which use aggregate data, often confirm the hypothesis of utlity maximizing behaviour subject to a budget constraint. It is remarkable that studies, which investigate the axiom of revealled preference, show that macro consumption data satisfy the utility maximization hypothesis in the same way, or even better, as micro consumption data does. This in spite of results, given in Gorman (1953, 1959) and Muellbauer (1976), which show that the requirements for the existence of a representative consumer after aggregation over goods and individuals are stringent.

This cluapter investigates the construction of aggregate consumption data by using price undex numbers. The main subject is the influence of aggregation on the existence of a rationalizing utility function, or even a homothetic rationalizing utility function. Of course such an analysis has also a meaning for aggregate production data. In that case the subject is the existence of a production function, which satisfies the hypothesis of cost minimization; assuming that production levels are unknowm.

The problem is sufficiently complicated to leave the theoretical approach and attack it with data experiments. This approach is suggested in Fisher (1971), and Fisher, Solow and Kearl (1977); where aggregate Cobb-Douglas and CES production functions were investigated. They used input-output data of simple fictitious economies, consisting of several cost minimizing producers. They estimated production functions for the aggregate data, using the hypothesis of cost minimization. Although the necessary conditions for aggregation were violated, the estimated production functions fitted well to the aggregate data and the wage prediction was good. The investigation in this chapter differs from Eisher's aggregation problem, in the sense that aggregation over goods is investigated, instead of aggregation over individuals. Furthermore, the analysis is based on nonparametric methods instead of parametric methods.

This chapter has the following contents. First a summary of related empirical research is given. Then a short description of several commonly used aggregation methods. These aggregation methods are used to aggregate Dutch consumption data and ranclom clata. Nonparametric tests are applied to check whether the aggregate data are compatible with the existence of aggregate rationalizing utility functions.

\subsection{Empirical Results}

The previous chapters contain empirical results for Dutch consumption data. In this section I give an overview of several other empirical studies in which the axiom of revealed preference, and the existence of homothetic utility functions, is tested.

Koo $(1963,1971)$, and Koo and Hasenkamp (1972) use consumer food panel data of 250 households with which they have empirically tested the axiom of revealed preference. They conclude that nearly every family made a relatively small number of inconsistent choices. Mossin (1972) tests the axiom of revealed preference with data based on consumers" reports about weekly purchases of everyday commodities. He compares the individual demand functions with the mean demand function and concludes that the mean demand 
function agrees bietter with the axiom of revealed preference. Maks (1978, 1980, 1982, 1984), Landsburg (1981) and Varian (1982) investigate aggregate consumption data for the Netherlands and Germany, the United Kingdom, and the United States respectively. They find hardly any violations of the axion of revealed preference.

Other empirical studies, concerming the existence of homothetic utility functions, are presented in Diewert and Parkan (1978), and Manser and McDonald (1988). In accordance with the results in Chapter 3 , these studies show that annual aggregate consumption data are often nearly consistent with homothetic utility maximization.

\subsection{Explanations for the Empirical Results}

In literature several explanations are given for the question why aggregate consumption data may agree better with the hypothesis of utility maximization than micro consumption data do. Of course one has to distinguish several kinds of aggregation. There is aggregation from individual quantity choices to quartity choices of a representative consumer. Secondly, there is aggregation of the data from shorter periods to longer periods. In the third place, there is the joining of the commodities in commodity groups, and aggregation of prices and quantities of these groups into aggregated group prices and quantities. Each of these three types of aggregation may be a cause for better agreement of macro consumption data with the hypothesis of utillity maximization.

The usilal argument to explain why macro consumption data are consistent with the axiom of revealed preference is the presence of an upward trend in income. As argued in Chapter 2, such a trend tends to bide inconsistent choices. However, there is no upward trend in income present in the pre-war data used in Maks $(1978,1980)$, which appeared to be consistent with the assumption of utility maximization. Also, as is shown in Chapter 3, an upward trend in income is no valid argument to explain why consumption data are nearly, or even completely, consistent with homothetic utility maximizing behaviour. Some other arguments shall be considered in the following.

To explain that more inconsistent choices occur in weakly consumption data. than in yearly data, Koo (1963) mentions seasonal influences, which may cause a taste change. In a later article Koo and Schmidt (1974) argue that inconsistent choices may be caused by the inability of consumers to rank very dissimilar alternatives in a consistent way. Mossin (1968) remarks that there may be stochastic elements in the behaviour of the consumer, which are not as strongly present in the mean demand function of the total population as in individual demand functions. One may also apply this argument to explain the occurrence of less inconsistencies in consumption data, which are aggregated over longer periods.

The above arguments are concerned with aggregation over time and individuals. This chapter investigates influence of the third aggregation type, price and quantity aggregation, on the existence of a rationalizing utility function. Before starting this investigation $\mathrm{I}$ will specify the price and quantity aggregation methods which are investigated.

\subsection{The Use of Price Index Numbers}

Often one wishes to represent data for a group of commodities as data for one single aggregate commodity. This means that the observations $\left(p^{i}, q^{i}\right)$, $i=1, \ldots, m$, representing the price and quantity vectors for the commodity group, will be represented by a series $\left(P_{i}, Q_{i}\right), i=1, \ldots, m$, representing the 
prices and quantities for the aggregate commodity. This is usually done by the choice of a price index, which maps the series $\left(p^{i}, q^{i}\right), i=1, \ldots, m$, on a series price index numbers $P_{i,}=1, \ldots, m$. Then, using the assumption that $p^{i} q^{i}=P_{i} Q_{i}$, the quantities $Q_{i}$ for the aggregate commodity are defined by

$$
Q_{i}=p^{i} q^{i} / P_{i} \quad(i=1, \ldots, m) .
$$

In Table 6.1 definitions are given of commonly used price indices. In this table the price index $P_{b c}$ measures the change of price vector $p^{c}$, for current period $c$, relative to price vectior $p^{b}$, in base period $b$.

Table 6.1 Price Index Niumbers

$\begin{array}{ll}\text { Laspeyres } & L_{b c}=p^{c} q^{b} / p^{b} q^{b} \\ \text { Paasche } & 1 / L_{c b}=p^{c} q^{c} / p^{b} q^{c} \\ \text { Fisher } & \left(L_{b c} / L_{c b}\right)^{1 / 2} \\ \text { Arithmetic } & A_{b c}^{w}=\sum_{n} \beta_{n}^{w} p_{n}^{c} / p_{n}^{b}, \text { where } \beta_{n}^{w}=p_{n}^{w} q_{m}^{w} / p^{w} q^{w} \\ \text { Geometric } & G_{b c}^{w}=\prod_{n}\left(p_{n}^{c} / p_{n}^{b}\right)^{\beta_{n}^{w}}, \text { where } \beta_{n}^{w w}=p_{n}^{w} q_{n}^{w} / p^{u b} q^{w} \\ \text { Törnquist } & \left(G_{b c}^{b} / C_{c b}^{c}\right)^{1 / 2}\end{array}$

In the arithmetic and geometric index a weight period $w$ is used, for which the price and quantity vector $\left(p^{w}, q^{w}\right)$ supplies the weights $\beta_{n}^{w^{w}}=p_{n}^{w} q_{n}^{w} / p^{w} q^{w{ }^{*}}$, often the base period is chosen as weight period. In that case the arithmetic index is equal to the Laspeyres index $A_{b c}=L_{b c}$

To derive a price index number for all periods, one may choose the first period as a fixed base period. Then $P_{\Perp i}$ provides a price index for all periods 2 . Such an index is called a direct index. Another kind of index is the chained index. This means that the index $C_{i}$, for a time series $\left(p^{1}, q^{1}\right),\left(p^{2}, q^{2}\right), \ldots$, is computed as

$$
C_{i i}=D_{12} D_{23} \cdots D_{i-2 i-1} D_{i-1 i} \text {, }
$$

using a chain of direct index numbers $D_{i j}$. In general one may use a subset of base periods $i_{1}, i_{2}, \ldots$, and compute the index numbers in the following way

$$
C_{i}=D_{1 i_{1}} D_{i_{1} i_{z}} \cdots D_{i_{i} i} \quad\left(1<i_{1}<i_{2} \cdots<i_{i}<i\right)
$$

Note that using the geometric or arithmetic price index number has an advantage, because computation of these indices is possible without having all quantity information avallable. Concerning these indices it is sufficient that we know the quantity vector $q^{u *}$ and nominal expenditures $p^{w} q^{u}$ for the weight periods.

For the geometric index we have $G_{12}^{t \prime} G_{23}^{w}=G_{13}^{u}$ and - using a fixed weight period - there is no difference between the chained and direct geometric index.

\subsection{Results for Aggregate Duteh Consumption Data}

To investigate the effect of price and quantity aggregation for consumption data, the Dutch consumption data are aggregated to several aggregation levels, using the various price indices mentioned in Table 6.1. A detailed description of the 4,12 and 26 aggregation level is given in the publication 
of the Netherlands Central Bureau of Statistics (1981), which supplied the data set. The chained arithmetic index is computed with the first observation period as weight period; both the chained and direct geometric index are computed with the base period as weight period.

Each resulting data set is tested for the existence of a rationalizing utility function. It appeared that the method of aggregation had no effect on the results. I obtained in all cases exactly the same results as described in Chapter 2 for the original data set of 106 commodities. After this for each data set the smallest subset of $n$ observations is computed, which have to be deleted in order to be consistent with the existence of a rationalizing homothetic utility function. Furthermore, the maximal homothetic afficiency level $e$ was computed for each data set, using the efficiency transformation given in Example 2.3.

Table 6.2 Homotheticity Results

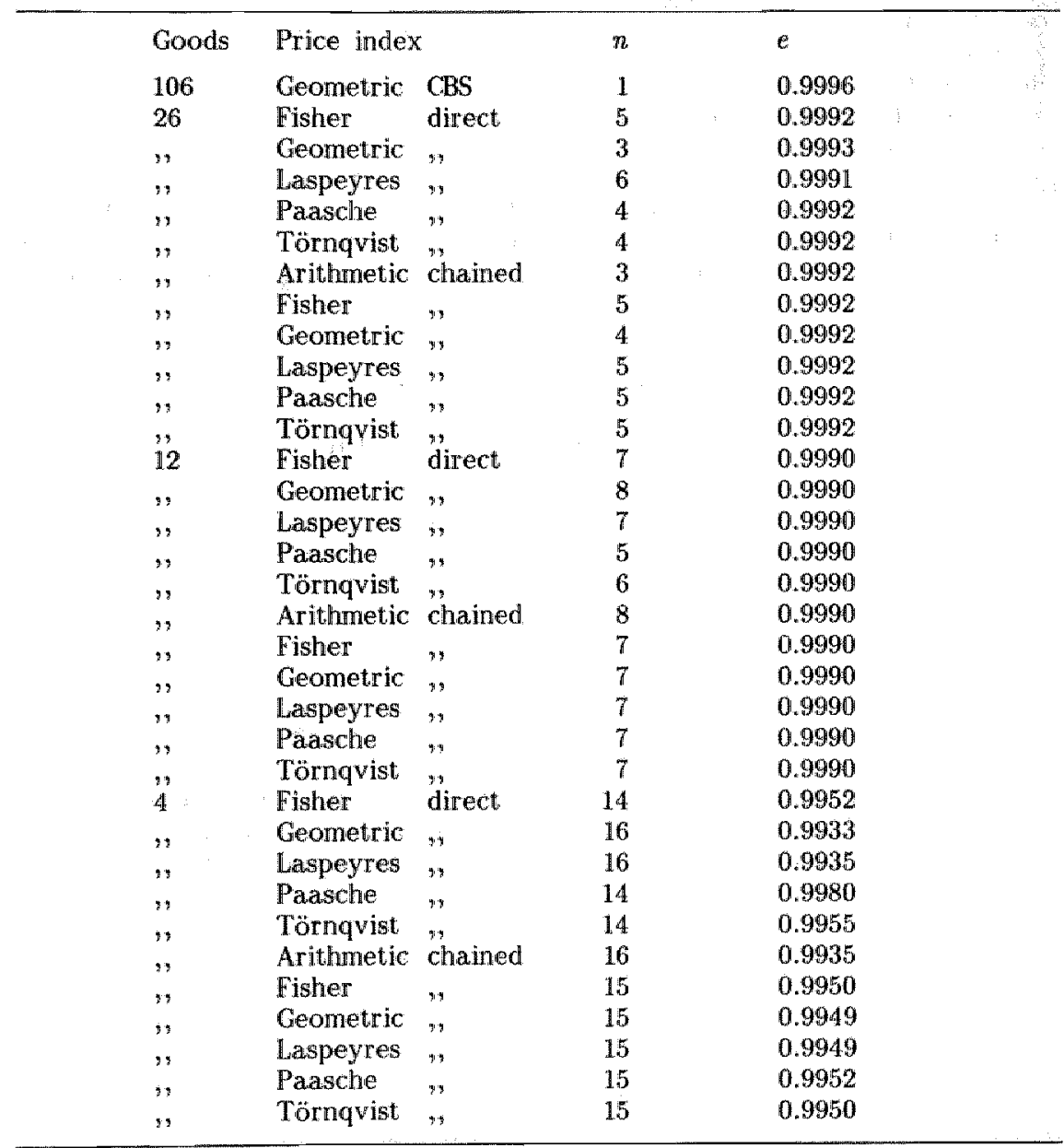

The results, presented in Table 6.2, have three characteristic properties. First, it appears that the homotheticity hypothesis is increasingly violated for higher aggregation levels. Secondly, although the efficiency levels are 
lower for higher aggregation levels, the efficiency levels remain rather close to one. In the third place, the choice of price index has no substantial influence on the results. Especially the chained indices appear to generate similar results. Before evaluating these findings, first other results, derived from iandom data, are presented.

\subsection{Results for Aggregate Random Data}

This section considers aggregate random data instead of actual consumption data, and we shall investigate what effect price and quantity aggregation may have. The aggregate Dutch consumption data in the previous section were obtained from a publication of the Netherlands Central Bureau of Statistics (1981). These data were aggregated by the Central Bureau of Statistics, using consumption data for more than a thousand commodities. In this section their aggregation scheme will be applied to random data.

To clarify the way the aggregate random data sets are constructed, the aggregation scheme of the Netheriands Central Bureau of Statistics is shortly described. To aggregate the Dutch consumption data at a 106 commodity level, the chained geometric price index numbers are computed, using several weight years. For the computation of these index numbers, a budget survey is needed for each weight year (see Section 6.4). In Table 6.3 the weight years $w$ are given at the left and at the right side appear the years in which the weight year $w$ is used to compute the direct geometric index $G_{i-1}$. Actually the 1960 budget survey covered the period 1959/1960. To keep things simple, we assume here that this burlget survey represents non-aggregate data in the year" 1960 .

Table 6.3 Weight Periods

$\begin{array}{ll}\text { Year of budget survey } & \text { Period of use } \\ 1951 & 1951-1961 \\ 1960 & 1962-1964 \\ 1964 & 1965-1969 \\ 1969 & 1970-1975 \\ 1975 & 1976-1977\end{array}$

To construct the aggregate random data, random prices and quantities are generated for 1060 commodities at each year in the period 1951-1977. Prices were chosen from a uniform distribution on $[1,3]$ and quantities from a uniform distribution on $[0,1]$. The random data were divided in 106 commodity groups of 10 commodities each. These groups were aggregated by several aggregation methods. I computed chained arithmetic and geometric price index numbers, using the aggregation method of the Netherlands Central Bureau of Statistics, which is described above. In addition Laspeyres, Paasche, Fisher, and Törnquist price index numbers were computed, using a similar aggregation method. Now the index numbers are computed as a chain of direct index mumbers, where the years of budget survey serve as the base periods in the chain. Thus we have for these indices

$$
C_{i}=D_{1 w_{1}} D_{w_{1} w_{2}} \ldots D_{w_{i}} \quad\left(1<w_{1}<w_{2^{*}} \ldots<w_{l}<i\right),
$$

where $w_{j}$ represent the years of the budget survey (see also Section 6.4). Furthermore, series of direct and chained price index numbers are computed. as was done in the previous section. The three aggregation methods will be referred to as CBS, direct and chained. 
Table 6.4 displays the results for the aggregate random data. from this table the following is apparent. Furst, the aggregate random data are nearly all cases consistent with the axion of revealed preference Even more surprising is the fact that the resulting aggregate data is in some cases consistent wh the existence of a rationalizing homothetic function. It is striking that the arithmetic and geometric price index, used by the Netherlands Central Bureau of Statistics, is one of these examples. Secondly, all efficiency levels are close to one. The Fisher and Tornqvist price indices preserve the random behaviour in the aggregate data sets in a better way, but there is hardly any difference in the homothetic efficiency level. In the third place, in contrast to earlier results for the Dutch consumption data, the choice of price index has substantial influence on the results. Even the chained indices generate different results.

Table 6.4 Results for Aggregate Random Data

\begin{tabular}{|c|c|c|c|c|c|c|}
\hline \multirow[b]{2}{*}{ Goods } & \multirow{2}{*}{\multicolumn{2}{|c|}{ Price index }} & \multirow{2}{*}{\multicolumn{2}{|c|}{ Any function }} & \multicolumn{2}{|c|}{ Homothetic } \\
\hline & & & & $e$ & $n$ & $e$ \\
\hline 1060 & Random & & 9 & 0.9908 & 21 & 0.9858 \\
\hline 106 & Fisher & direct & 0 & 1. & 17 & 0.9954 \\
\hline$n$ & Geometric &, & 0 & 1 & 4 & 0.9974 \\
\hline 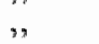 & Laspeyres & $"$ & 0 & 1 & 10 & 0.9968 \\
\hline 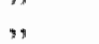 & Paasche & 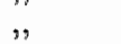 & 2 & 0.9993 & 11 & 0.9961 \\
\hline 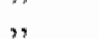 & Törnquist & 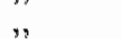 & 1 & 0.9999 & 16 & 0.9954 \\
\hline 4 & Arithmetic & chained & 0 & 1 & 0 & 1 \\
\hline$\because$ & Fisher & , & 2 & 0.9996 & 11 & 0.9974 \\
\hline$\because$ & Geometric & $"$ & $\overrightarrow{0}$ & 1 & 2 & 0.9999 \\
\hline 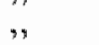 & Laspeyres & $"$ & 0 & 1 & 2 & 0.9992 \\
\hline 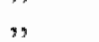 & Paasche & 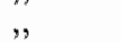 & 0 & 1 & 6 & 0.9986 \\
\hline 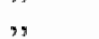 & Tơrnquist &, 3 & 2 & 0.9991 & 10 & 0.9975 \\
\hline 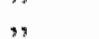 & Arithmetic & CAS & 0 & 1 & 0 & 1 \\
\hline " & Fisher & $"$ & 2 & 0.9991 & 15 & 0.9963 \\
\hline, & Geometric & $"$, & 0 & 1 & 0 & 1 \\
\hline$n$ & Laspeyres & , & 0 & 1 & 3 & 0.9989 \\
\hline 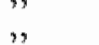 & Paasche & $"$ & 0 & 1 & 4 & 0.9972 \\
\hline$"$ & Törnquist &, & 3 & 0.9990 & 14 & 0.9966 \\
\hline
\end{tabular}

\subsection{Evaluation and Some More Results}

Bronars (1987) shows that nonparametric tests of preference maximization using per capita data are quite powerful against the alternative of random behaviour. However, from the results above it appears that the hypothesis of rational demand leads not to stringent estrictions on consumption datia that are aggregated over commodities. Moreover, even the restriction to homotheticity is not stringent in case the consumption data contain many commodities. This conclusion is supported by the empirical results in Diewert and Parkan (1978), and Manser and McDonald (1988). An important question we have to answer in this respect is the question why aggregation of random data may generate rational, and even homothetic rational, consumer behaviour. The obvious reason for this finding is the way the aggregate quantity measures were computed. Each aggregate quantity measure was computed as the division of the group budget by the group price index. Hence, the movement of the aggregate quantity measures will tend to be opposite to the movement of the price indices. So, commodity aggregation of random data introduces a tendency to economical behaviour. 
In order to investigate if the superposition principle is a sufficient explanation for our results, the following test is made. First, to independent tandom data sets are generated. From these two data sets aggregate data are constructed, using price index numbers derived from one raindom data set and expenditures derived from the second random data set. The resulting data were tested for rational demand $A$ result was derived, which confing our conjecture but in a stronger way than expected. The constructed aggregate data were consistent with the hypothesis of efficient rational homothetic demand for any type of used price index number. This result is disturbing, because it implies that unreliable independent sources of aggregate price index numbers and expenditures, may cause aggregate data to satisfy the hypothesis of rational, and even homothetic rational, demand.

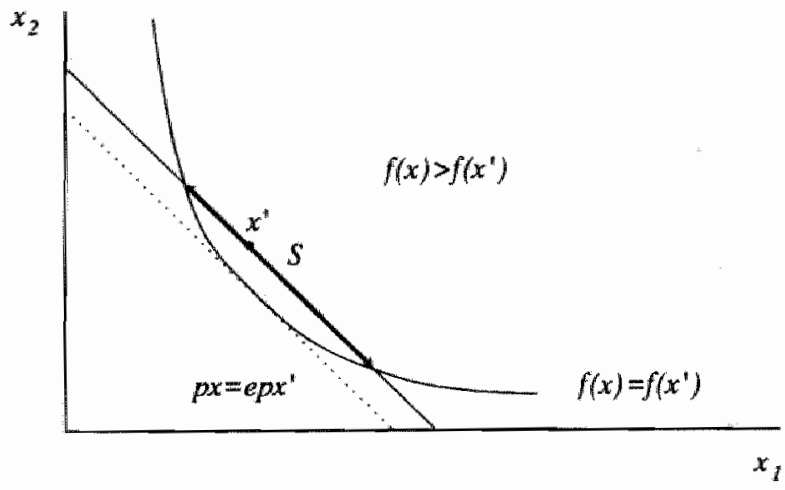

Figure 6.1 Inefficient Consumer Demand

A second point is the increasing violation of the homotheticity hypothesis at highel aggregation levels. This may be because the degree of freedom to choose a rationalizing linearly homogeneous utility function diminishes if there are less commodities in the data set. An example of this is a set of consumption data for one commodity. In that case there is no freedom of choice at all to choose the relative function values of a rationalizing linearly homogeneous atility function. To check the hypothesis of a diminishing degree of freedon, the 1060 commodity random data from Table 6.4 are aggregated to higher aggregation levels. The results obtained for the resulting data are similar to the results concerning the aggregate Dutch consumption data ${ }$ thus it appears that there is indeed a diminishing degree of freedom. However, there was one difference with the aggregate Dutch consumption data. The homothetic efficiency levels were closer to one at a higher aggregation level for all aggregate random data sets. An explanation for this may be a relative lack of variation in the data at a higher aggregation level, caused by the fact that all random prices and random quantities are chosen from the same probability distribution.

The next point is the occurrence of high efficiency levels, even when many observations are inconsistent with the hypothesis of efficient rational demand. Figure 6.1 shows an area $S$ of quantities which are consistent with $a$ 
rationalizing utility function of a certain efficiency level. This area increases fast when the efficiency level is lowered, assuming the indifference curve is not sharply curved. So we have to be careful when data are biased in such a way that it is easy to construct a smoothly curved utility function, approximately consistent with these data. For example in the case where price data are approximately linearly dependent. Then the existence of a rationalizing utility function for a high efficiency level does not exclude the possibility that many observations are inconsistent with the hypothesis of efficient rational demand.

It remains to explain the fact that all price index numbers for the Dutch aggregate data generate similar results, while the results for the random data depend substantially on the chosen price index. Especially the chained indices appear to generate similar results for the Dutch consumption data. An explanation for this result may be found in Vartia (1978) and Diewert (1978), who show that when prices and quantities do not differ too much between subsequent periods then both the Fisher and Törnqvist chained price indices yield approximately the same value. It seems that this argument applies to the other chained index numbers as well, and less to the direct indices, Clearly the random data do not consist of smoothly varying data, which explains the dependence of the random data results on the choice of price index. The fact that the Fisher and Tornqvist price indices preserve the random behaviour in the aggregate random data sets in a better way than the other indices is probably because these indices are not as strongly biased as the other indices. Both indices are of the form $\left(P_{b c} / P_{c b}\right)^{1 / 2}$, for which the upward, or downward, bias of price index $P_{b c}$ will be neutralized by a bias in opposite direction of $1 / P_{c b}$.

\subsection{Summary}

The issue in this chapter was the investigation whether or not aggregation may introduce economic behaviour in the resulting data sets. It is evident from the outcomes of the nonparametric tests for consistency, homotheticity and efficiency, that this is indeed the case. An explanation for these results is the application of the superposition principle, which implies that division of the budget by the price index results in the corresponding quantity measure. This introduces a tendency for the aggregate group quantity measures and the aggregate price index numbers to move in opposite directions. Lower prices are accompanied by higher quantities, which is just what one would expect from a rational consumer.

The results show that one has to be careful in drawing conclusions from aggregate data. The assumption that an obvious rule for rational demand is satisfied, price times quantity equals expenditures, has as implication that aggregate random data may tend to rational demand. The bellef in rational demand may be a self-fulfilling prophecy. Summarizing the above, one rnight state that the phrase What does it matter which index you use, what matters is that it works - which I have noticed in a book about indices - appears to contain unexpected truth. Aggregate consumption data may represent economic behaviour, even if the original data do not reflect economic behaviour at all. Moreover, the worst price index number, by accident computed from an other independent set of consumption data, may work very well. For random data I found that such price index numbers may generate aggregate data, which are fully consistent with the hypothesis of efficient rational homothetic consumer demand. 


\section{PART II \\ PRODUCER DEMAND}

\section{INTRODUCTION}

\subsection{Theory of Producer Behaviour}

Producer theory and consumer theory overlap each other. In this book we confine ourselves to producer behaviour for a single-output production function. The mathematical problems for a single-output production function in the producer theory and the utility function in the consumer theory are often arialogous. Differences between textbook producer and consumer theory are the following. First, produced output may be observed, while consumer utility is not observed. Another difference is that, while in standard consumer theory the utility function is not subject to change, the production function may change, because of technical progress. Furthermore, one may assume that the production function is a frontier production function, which represents a limiting frontier of the production output. This assumption allows the occurrence of technically inefficient production. A last point of difference will be treated later on: besides that a producer is a cost minimizer, a producer may also be a profit maximizer.

\subsection{Producer Demand}

The used definition of producer demand will be equivalent to consumer demand. However, in contrast to consumer behaviour, where utility is not observed, we can observe produced output. Therefore, I will often assume that producer data include the produced output $f(x)$. In that case we will observe $\left(c, x^{\prime}, f\left(x^{\prime}\right)\right)$, instead of $\left(c, x^{\prime}\right)$, and we will obtain the following slightly
different version of the consumer demand definition. Definition: For a function $f: X \rightarrow \mathbb{R}$, the set of producer demand with observed
output, is defined as

$$
\mathcal{D}_{d}^{o}(f)=\left\{\left(c, x^{\prime}, y\right) \mid\left(c, x^{\prime}\right) \in \mathcal{D}_{d}(f), y=f\left(x^{\prime}\right)\right\} \text {. }
$$

Furthermore, $D_{u}^{o}(f)$ etc. are delined in the same way. One has for example:

$$
D_{u}^{\prime}(f)=\left\{\left(c, x^{\prime}, y\right) \mid\left(c, x^{\prime}\right) \in \mathcal{D}_{u}(f), y=f\left(x^{\prime \prime}\right\}\right. \text {. }
$$

\subsection{Technical Inefriciency and Frontier Production Functions}

Given a production function $f: X \rightarrow \mathbb{R}$, we assumed until now that the relation between input $x$ and output $y$ is simply described by $y=f(x)$. A more general description of producer behavioun allows for technical inefficiency. In that
case the relation is described by

$$
\{(x, y) \| x \in X, y \leq f(x)\} \text {. }
$$
Thus, due to technical inefficiency, produced output may also be less than
the maximal possible output. The production function represents now the
best-practice frontier of best-practice frontier of production, and is called a frontier production 
function. We define producer behaviour with observed output in the following way for frontier production functions.

Definition: For a frontier production function $f: X \rightarrow R_{R}$ the set of producer demand with observed output, is defined as

$$
D_{d}^{f}(f) \equiv\left\{\left(c, x^{\prime}, y\right) \mid\left(c, x^{\prime}\right) \in D_{d}(f), y \leq f\left(x^{*}\right)\right\} \text {. }
$$

Furthermore, $\mathcal{D}_{w}^{f}(f)$ etc. are defined analogously. For example

$$
\mathcal{D}_{u}^{f}(f) \equiv\left\{\left(c_{n} x^{\prime}, y\right) \mid\left(c, x^{\prime}\right) \in \mathcal{D}_{u}(f), y \leq f\left(x^{\prime}\right)\right\}
$$

\subsection{Technical Change}

The method of production may change over time. New technical methods are introduced, which may increase production. Therefore one often supposes that the production function is changing over time. I will call such a change of the production function technical change. In the most general description of such a change one uses several production functions: one for each observation. period. A more specific definition of technical change is given by Hicks.

Definition: Hicks neutral change, for a series $\left\{t_{i} \in \mathbb{R}_{+}\right\}_{i \in l}$ and a production function $f: X \rightarrow \mathbb{R}$, results in a series of production functions $\left\{f_{i}: X \rightarrow \mathbb{R}\right\}_{i \in I}$ given as

$$
f_{i}(x)=t_{i} f(x) \quad, \quad(i \in I, x \in X) .
$$

Thus for each period the output is simply multiplied by a positive factor. In a similar way we may multiply inputs of the production function, which leads to the following definition.

Definition: Given a production function $f: X_{1} x_{2} \rightarrow \mathbb{R}$. Neutral change of imput $X_{2}$ for a series $\left\{t_{i} \in \mathbb{R}_{+}\right\}_{i \in I}$ results in the series of production functions $\left(f_{i}: X_{1} \times X_{2} \rightarrow \mathbb{R}\right\}_{i \in I}$ given as

$$
f_{i}\left(x_{1}, x_{2}\right)=f\left(x_{1}, t_{i} x_{2}\right) \quad\left(i \in I, x_{1} \in X_{1}, x_{2} \in X_{2}\right)
$$

There are two special cases of neutral change. When $X_{2}$ represents labour one has Harrod neutral change, and when $X_{2}$ represents capital one has Solow neutral change.

An obvious hypothesis concerning changing production functions is the assumption that production is improving over time. In that case the production functions are increasing over time. In that case I replace the term change by the term progress in the above definitions.

Definition: Technical progress for a series production functions $\left\{f_{i}\right\}_{i e}$ means that we have $f_{j} \leq f_{i}$ if $j \leq i$. In a similar way we define (Hicks) neithal progress.

\subsection{Rationalizing Production Functions}

In this chapter I will consider the following kinds of data:

1. Data where output is not observed, like $D_{d}$

2. Data where output is observed, like $D_{d}^{o}$.

3. Data where output is observed and limited by a frontier production function, like $\mathcal{D}_{d}$.

For all these data definitions we may define the set of functions which rationalizes producer behaviour.

Definition: Let $D$ be a data set of producer behaviour. The family of production functions $\mathcal{F}_{d}^{*}$, which rationalizes $D$ for producer denand with 
observed output, is defined as

$$
\mathcal{F}_{d}^{O}(D) \equiv\left\{f: X \rightarrow \mathbb{R} \mid D \subset \mathcal{D}_{d}^{o}(f)\right\} \text {. }
$$

The definition for $\mathcal{F}_{d}^{f}$ etc. are analogous.

Just in casse of $\mathcal{F}_{d}$ for consumer demand one has

$$
\mathcal{F}_{d}^{-a}\left(D_{1} \cup D_{2}\right)=\mathcal{F}_{d}^{\infty}\left(D_{1}\right) \cap \mathcal{F}_{d}^{\infty}\left(D_{2}\right)
$$

This is in general valid for any kind of behaviour, like $\mathcal{F}_{d}^{f}$, which is defined in the same way as $\mathcal{F}^{*}$

\subsection{Application to Empírical Data}

In the following chapters of Part II, Producer Demand, the above definitions are applied to develop a theory in the same way as is done in Part I for constmer demand. By way of illustration the theory is applied to a data set of Dutch producer behaviour. The nominal values concerning this data set are derived from input-output tables of the Dutch economy, covering the years $1969-1983$, as published by the Netherlands Central Bureau of Statistics. For some years additional information was obtained from this bureau to get a consistent array of tables. To obtain prices for the nominal data, various sources were used. The large tables were aggregated to a less detalled sectior format used by the Netherlands Central Planning Bureau. This made it possible: to use less accurate sector price data, supplied by this bureau, for the missing prices. The way capital is treated will be discussed an Part IV, Capital. Here it is sufficient to know that there is a price and quantity for each good, including capital. For a more detailed description of the Dutch industry data I refer the reader to Appendix E.

\subsection{Contents of Part II}

Chapter 8 is concerned with the existence of a production function for given producer data. This chapter treats all variations of the producer demand problem that are mentioned above.

Chapter 9 is concerned with linearly homogeneous production functions for given producer data. Results are derived by searching for the upper bound of all rational linearly homogeneous production functions for the given data set. The application of the resulting theory to the Dutch industry data yields interesting graphs of, for example, technical efficiency upper bounds, bounds for the frontier production function and Hicks neutral progress.

Chapter 10 is a short chapter containing some results for homothetic production functions, i.e a monotonic transformation of a linearly homogeneous function. This chapter is concermed with the existence of such a function for given producer data with observed output. Moreover, it treats restrictions on the underlying linearly homogeneous function.

Chapter 11 treats weakly separable production functions. This chapter is concerned with the problem whether given producer demand data, including observed output, may be generated by a weakly separable production function. These results are extended to linearly homogeneous weakly separable functions and weakly separable functions whth a linearly homogeneous subfunction. The problem of technically progressing weakly separable production functions is not considered, because the results for weakly separable productions functions - without the addition of technical progress - are already
complicated. 


\section{PRODUCER DEMAND}

\subsection{Introduction}

This chapter considers the question whether there exists a production function for given producer data without any a priori restriction on the production function. This problem is considered for producer demand, frontier production functions and technical change. Furthermore, I will derive nonparametric restrictions on the technical efficiency coefficients and the scale of neutral progress. It turns out that for the Dutch industry data there only are slight violations of these nonparametric restrictions. This result is not very surprising, because there is a large set of production functions from which we may choose. The results are derived without using any restriction on the production function. Later on in the following chapters, when we consider restrictions on the production function, we shall encounter nonparametric conditions that are severely violated.

\subsection{Producer Demand}

There is no difference between producer and consumer demand when produced output is not observed. This means that in that case the axiom of revealed preference can be used to test the hypothesis of producer demand. This follows directly from Theorem 2.3, which states that the existence of a utility function for consumer demand can be tested with the axiom of revealed preference. The Dutch industry data without produced outputs accept the existence of a production function.

Since the produced outputs of the Dutch industry are available a second test can be made, which checks whether these outputs are consistent with the axiom of revealed preference. For such a test the following theorem can be used.

Theorem 8.1: Suppose $D=\left\{\left(c_{i}, x^{i}, y_{i}\right)\right\}_{i \in I}$ is a finite data set, where $c_{i}: X \rightarrow \mathbb{R}_{+}$. Then the following conditions are equivalent:

(i) $\mathcal{F}_{d}^{a}(D) \neq \emptyset$.

(ii) One has

$$
\begin{array}{ll}
c_{i}\left(x^{j}\right) \leq c_{i}\left(x^{i}\right) \Rightarrow y_{j} \leq y_{i} & (i, j \in I), \\
c_{i}\left(x^{j}\right)<c_{i}\left(x^{i}\right) \Rightarrow y_{j}<y_{i} & (i, j \in I) .
\end{array}
$$

Proof (i) $\Rightarrow($ ii $)$ : Obvious, use the definition of $F_{d}^{o}$.

$($ ii) $\Rightarrow(i):$ By Theorem 2.4 there exists a function

$$
f(x)=\min _{i \in I} f_{i}+\lambda_{i}\left[c_{i}(x)-c_{i}\left(x^{i}\right)\right] \quad(x \in X)
$$

such that $\lambda_{i}>0$ and $f\left(x^{i}\right) \geq f_{i}$ for all $i \in I$, and

$$
f_{i} \leq f_{j} \Leftrightarrow y_{i} \leq y_{j} \quad(i, j \in I) \text {. }
$$

So, because clearly $f\left(x^{i}\right) \leq f_{i}$, one has $f\left(x^{i}\right)=f_{i}$ for all $i \in I_{\text {, }}$ and it is not difficult to show that we have $\left(c_{i} x^{i}\right) \in D_{d}(f)$ for all $i \in I$. Thus, for any strictly increasing function $m: \mathbb{R} \rightarrow \mathbb{R}$, such that $m\left(f_{i}\right)=y_{i}$ for all $i \in l$, one has mof $\in \mathcal{F}_{d}^{a}(D)$. That such a function $m$ exists follows from

$$
f_{i} \leq f_{j} \Leftrightarrow y_{i} \leq y_{j} \quad(i, j \in I) .
$$

When the above theorem is applied to the Dutch industry data, to test the hypothesis of producer demand, it appears that two observations have to be 
eliminated.

Using the abowe theorem and the efficiency transformation given in Example 2.3, we can determine the economical efficiency upper bound for the complete data set. I computed this efficiency upper bound for the Dutch industry data, using bisection, and the result was $e=99.5 \%$.

\subsection{Frontier Production Functions}

Let $D=\left\{\left(c_{i}, x^{i}, y_{i}\right)\right]_{i \in I}$ be $a$ set of production data. For these data a frontier production function $f \in \mathcal{F}^{f}(D)$ satisfies by definition $f \in \mathcal{F}(D)$ and $f\left(x^{i}\right) \geq y_{i}$ for all $i \in I$. The corresponding values of technical efficiency are

$$
t_{i}=y_{i} / f\left(x^{i}\right) \quad(i \in I)
$$

So the technical efficiency values are maximal, for given production data, when the corresponding frontier production function output is minimal. Hence the following definition may be useful.

Definition: The lower bound $L$ is equal to

$$
L(F, x)=\inf _{f \in F} f(x) \quad(x \in X)
$$

Using this definition we may consider the maximal technical efficiency values for given production data.

Derinition: For given data $D=\left\{\left(c_{i}, x^{\mathrm{f}}, y_{i}\right)\right\}_{\text {ivel }}$, the technical efficiency upper bounds $t_{i}$ are defined as

$$
t_{i}=y_{i} / u_{i}, \quad u_{i}=L\left(\mathcal{F}_{d}^{f}(D), x^{i}\right) \quad(i \in I)
$$

Of course every technical efficiency upper bound will be less than or equal to one. The technical efficiency upper bounds provide a bound for the possible waste of output for producer demand. I will argue below how the problen may be solved..

The information needed to solve the problem, is provided by the axiom of revealed preference, which is described in Chapter 2 . In order that any solution $u_{i}$ exists we have to assume $\mathcal{F}_{d}(D) \neq 0$. When this assumption is satisfied, we can apply the axiom of revealed preference. Application of this axiom reveals information about the ordering of the values $f\left(x^{i}\right)$ for frontier production functions $f \in \mathcal{F}_{d}^{f}(D)$. This information is represented by the
relations

$$
\begin{array}{ll}
c_{i}\left(x^{j}\right) \leq c_{i}\left(x^{i}\right) \Leftrightarrow x_{i} R x_{j} & (i, j \in I), \\
c_{i}\left(x^{j}\right)<c_{j}\left(x^{i}\right) \Leftrightarrow x_{i} P x_{j} & (i, j \in I) .
\end{array}
$$

These revealed preference relations $R$ and $P$ and all the observed values $y_{i}$ contain together all the information, necessiary to determine the technical efficiency upper bounds. The following simple problem determines the minimal frontier production values $u_{i}$ from the revealed preference relations $R$ and $P$,
and all outputs $y_{i}$ :

$$
\text { inf } x_{i} \quad(i \in I)
$$

subject to

$$
\begin{array}{ll}
u_{i} \geq y_{i} & (\dot{i} \in I), \\
w_{i} \leq w_{j} & \left(\dot{i} j \in I: x_{i} R x_{j}\right), \\
u_{i}<w_{j} & \left(\dot{i}_{i} j \in I: x_{i} P x_{j}\right),
\end{array}
$$

Because we have $P \subset R$, the relation $P$ plays no part. In fact we may drop the last condition and obtain for finite data sets the following solution

$$
u_{i j}=\max \left\{y_{k} \mid k \in I: x^{k} R^{*} x^{i}\right\} \quad(x \in I) \text {. }
$$


Remark: The question is whether we can actually minimize all walues $u_{4}$ together. This is indeed the case for finte data sets, because $f(f D)$ is closed with respect to the pointwise minimum operation. Thus if $f_{i} \in F_{d}(D)$ for all $i \in I$ then $f \in \mathcal{F}_{d}^{f}(D)$, where

$$
f=\min _{i} f_{i}
$$

is the pointwise minimum over all functions $f$. This means that we can always find a function $f \in \mathcal{F}_{d}^{f}(D)$ that minimizes all values $u_{i}=f\left(x^{i}\right)$ together.

The next theorem is based on the above outline and uses this solution to determine the technical efficiency upper bounds.

Theorem 8.2: Suppose $D=\left\{\left(c_{i}, x^{i}, y_{i}\right)\right\}_{i \in I}$ is a finite data set and $\mathcal{F} d(D) \neq 0$. Let $R$ be the corresponding revealed preference relation, determined by

$$
x^{j} R x^{i} \Leftrightarrow c_{i}\left(x^{j}\right) \leq c_{i}\left(x^{i}\right) \quad(i, j \in I) .
$$

Then

$$
\left.L\left(\mathcal{F}_{d i}^{\mathcal{f}}(D), x^{i}\right)=\max \left\{y_{k} \mid k \in I: x^{k} R^{*} x^{i}\right\} \quad \forall i \in I\right) .
$$

Proof: Put

$$
u_{i}=\max \left\{y_{k} \mid k \in I: x^{k} R^{*} x^{i}\right\} \quad(i \in I) \text {. }
$$

First we show that one has

$$
u_{i} \leq L\left(\mathcal{F}_{d}^{f}(D), x^{i}\right) \quad(i \in I) .
$$

Let $f \in \mathcal{T}_{d}^{f}(D)$. To prove the inequality, it is sufficient to show that we have

$$
y_{k} \leq f\left(x^{i}\right) \quad\left(i, k \in I: x^{k} R^{*} x^{i}\right) \text {. }
$$

From $f \in \mathcal{F}_{d}(D)$ and the definition of consumer demand $\mathcal{F}_{d}$ and $R$, we obtain

$$
f\left(x^{k}\right) \leq f\left(x^{i}\right) \quad\left(i, k \in I: x^{k} R x^{i}\right),
$$

hence one has also $f\left(x^{k}\right) \leq f\left(x^{i}\right)$ in case $x^{k} R^{*} x^{i}$. So, because $f \in \mathcal{F}_{d}^{f}(b)$ and the definition of $\mathcal{F}_{d}^{f}$ implies $y_{k} \leq f\left(x^{k}\right)$, we have indeed

$$
y_{k} \leq f\left(x^{k}\right) \leq f\left(x^{i}\right) \quad\left(i, k \in I: x^{k} R^{*} x^{i}\right) .
$$

The proof is complete if we can show that we have

$$
u_{i} \geq L\left(\mathcal{F}_{d}^{f}(D), x^{i}\right) \quad(\dot{i} \in I)
$$

As one may verify, the numbers

$$
\left.w_{i}=\max \left\{y_{k} \mid k \in I: x^{k} R^{*} x^{i}\right\} \quad \mid i \in I\right)
$$

are alution of the problem

$$
\text { inf } u_{i} \quad(\dot{i} \in I)
$$

subject to

$$
\begin{array}{ll}
u_{i} \geq y_{i} & (i \in I), \\
u_{i} \leq u_{j} & \left(i, j \in I: x_{i} R x_{j}\right) .
\end{array}
$$

Now, since we assumed $\mathcal{T}_{d}(D) \neq \varnothing$, there is alution $f \in \mathcal{F}_{d}(D)$. For this function we may assume $f\left(x^{i}\right)>0$ for all $i \in I$, because every monotonic transformation of $f$ is element of $\mathcal{F}_{d}(D)$. Now we may verify that

$$
v_{i}=u_{i}+\delta f\left(x^{i}\right) \quad(i \in I),
$$

for arbitrary $\delta>0$, satisfies the conditions

$$
\begin{array}{ll}
v_{i} \geq y_{i} & (i \in I), \\
v_{i} \leq v_{j} & \left(i, j \in I: x_{i} R x_{j}\right), \\
v_{i}<v_{j} & \left(i, j \in I: x_{i} P x_{j}\right) .
\end{array}
$$


By condition (ii) in Theorem 8.1 , this implies

$$
\eta_{d}\left(\left\{\left(c_{i}, x^{i}, v_{i}\right)\right\}_{i \in I}\right) \neq \phi_{i}
$$

Furthermore, we have thus

$$
v_{i} \geq L\left(\mathcal{F}_{d}(D), x^{i}\right) \quad(i \in I)
$$

Hence, by lletting $\&$ approach to zero, we obtain

$$
w_{i} \geq L\left(\mathcal{F}_{d}^{f}(D), x^{i}\right) \quad(i \in I)
$$

Applying the above theorem to the Dutch industry data resulted in wo technically inefficient years." These years were 1975 with technical efficiency upper bound $98.4 \%$ and 1978 with $97.3 \%$.

\subsection{Technical Progress}

First I consider technical progress with observed output. An approach which I applied successfully in nonparametric problems concerning technical progress is the following. Consider a given data set $\left\{d_{i}\right\}_{i \in I}$ for the periods $i \in I$. Suppose we wish to extract nomparametric information in order to derive an upper bound for the possible values of the production function at a certain period $i \in l$. Then a part of the nonparametric information. derived for the similar case without technical progress, can be used. Because technical progress means that the production function is increasing, all upper bound information concerning the production functions in the past periods $j \leq i$ is relevant. This approach is used in the following theorem.

Theorem 8.3: Suppose $D=\left\{\left(c_{i}, x^{i}, y_{i}\right)\right\}_{\text {ier }}$ is a finite data set, where $c_{i}: X \rightarrow \mathbb{R}_{+*}$ Then the following condations are equivalent:

(i) There exists a series of production functions $\left(f_{i}\right\}_{i \in I}$ such that $f_{j} s f_{i}$ if $j \leq i$, and $\left(c_{i}, x^{i}, y_{i}\right) \in \mathcal{D}_{d}^{o}\left(f_{i}\right)$ for all $i \in I$.

(ii) One has

$$
\begin{aligned}
& c_{i}\left(x^{j}\right) \leq c_{i}\left(x^{i}\right) \Rightarrow y_{j} \leq y_{i} \quad(i, j \in I: j \leq i), \\
& c_{i}\left(x^{j}\right)<c_{i}\left(x^{i}\right) \Rightarrow y_{j}<y_{i} \quad(i, j \in I: j \leq i) .
\end{aligned}
$$

Proof $(i) \Rightarrow$ (ii): Follows immediately from the definition of $\mathcal{D}_{d}^{\infty}$.

$(i i) \Rightarrow$ (i): Suppose (ii) is satisfied. Then by Theorem 2.4 , we may choose numbers $\lambda_{i}>0$ and $\phi_{i}$, such that

$$
\begin{aligned}
& \phi_{i}+\lambda_{i}\left[c_{i}\left(x^{j}\right)-c_{i}\left(x^{i}\right)\right] \geq \phi_{j} \quad(i, j \in I: j \leq i) \text {, } \\
& \phi_{j} \leq \phi_{i} \Leftrightarrow y_{j} \leq y_{i} \quad\left(i_{j}, j \in I\right) \text {. }
\end{aligned}
$$

Let $m$ be a monotonic transformation such that $m\left(\phi_{i}\right)=y_{i}$ for all $i \in I$, and put

$$
f_{j}(x)=m\left(\min _{i \geq j} \phi_{i}+\lambda_{i}\left[c_{i}(x)-c_{i}\left(x^{i}\right)\right]\right) \quad(j \in I, x \in X)
$$

For these functions one can easily verify that condition (i) is satisfied. We have $f_{j} \leq f_{i}$ if $j \leq i$, because in general

$$
\min _{k \geq j} \phi_{k}+\lambda_{k}\left[c_{k}(x)-c_{k}\left(x^{k}\right)\right] \leq \min _{k \geq i} \phi_{k}+\lambda_{k}\left[c_{k}(x)-c_{k}\left(x^{k}\right)\right] \quad(x \in X, i, j \in I: j \leq i) .
$$

Furthermore, for every $B \in I$, we have $\left(c_{i}, x^{i}, y_{i}\right) \in \mathcal{D}_{d}^{o}\left(f_{i}\right)$, since $f_{i}\left(x^{i}\right)=m\left(\phi_{i}\right)=y_{i}$ and moreover $\left(c_{i}, x^{i}\right) \in \mathcal{D}_{d}\left(f_{i}\right)$, because

$$
\begin{array}{lll}
c_{i}(x) \leq c_{i}\left(x^{i}\right) & \Rightarrow f_{i}(x) \leq m\left(\phi_{i}+\lambda_{i}\left[c_{i}(x)-c_{i}\left(x^{i}\right)\right]\right) \leq m\left(\phi_{i}\right)=f_{i}\left(x^{i}\right) & (i \in I) \\
c_{i}(x)<c_{i}\left(x^{i}\right) \Rightarrow f_{i}(x) \leq m\left(\phi_{i}+\lambda_{i}\left[c_{i}(x)-c_{i}\left(x^{i}\right)\right]\right)<m\left(\phi_{i}\right)=f_{i}\left(x^{i}\right) & (i \in I) .
\end{array}
$$

To satisfy the hypothesis of producer demand with technical progress, one had to eliminate one observation from the Dutch industry data for the whole data set I derived as upper bound of economical efficiency $e=99.8 \%$, using the efficiency transformation of Example 2.3. 
Now, we may consider the case where output is not observed. Then, ass stated in the theorem given below, the hypothesis of technical progress can never be falsified. To test the hypothesis we have to check the existence of numbers $y_{i}$ satisfying condition (ii) in Theorem 8.3 above. I will show that such numbers always exist. This will be shown while prowing the following theorem.

Theorem 8.4: Suppose $D=\left\{\left(c_{i}, x^{i}\right)\right\}_{i \in I}$ is a fante data set. Then there exists a series of production functions $\left\{f_{i}\right\}_{i \in I}$ such that $f_{j} \leq f_{i}$ if $j \leq i$ and $\left(c_{i}, x^{i}\right) \in D_{d}\left(f_{i}\right)$ for all $i \in I$.

Proof: The proof is based on the axiom of revealled preference, which is modified for the case of technical progiess. As described in Theorem 8.3, we have the following information when there is technical progness

$j$ is not preferred to $i$ if $c_{i}\left(x^{j}\right) \leq c_{i}\left(x^{i}\right) \quad(i, j \in I: j \leq i)$

$i$ is preferred to $j$ if $c_{i}\left(x^{j}\right)<c_{i}\left(x^{i}\right) \quad(i, j \in I: j \leq i)$.

So we can define the revealed preference relations $R$ and $P$ for technical progress as

$$
\begin{array}{ll}
x^{j} R x^{i} \Leftrightarrow c_{i}\left(x^{j}\right) \leq c_{i}\left(x^{i}\right) \text { and } j \leq i & (i, j \in I), \\
x^{j} P x^{i} \Leftrightarrow c_{i}\left(x^{j}\right)<c_{i}\left(x^{i}\right) \text { and } j \leq i & (i, j \in I) .
\end{array}
$$

By Theorem 8.3 there is a series of technically progressing functions for the data if we can find numbers $y_{i}$, such that one has

$$
\begin{array}{ll}
x^{j} R x^{i} \Rightarrow y_{j} \leq y_{i} & (i, j \in I), \\
x^{j} P x^{i} \Rightarrow y_{j}<y_{i} & (i, j \in I) .
\end{array}
$$

As described in Chapter 2, such numbers do exist when the axiom of revealed preference $R^{*} \cap P c=\emptyset$ is satisfied. This, however, is always the case, because we have

$$
\begin{aligned}
& x^{j} R^{*} x^{i} \Rightarrow j \leq i \quad(i, j \in I) \\
& x^{j} P^{c} x^{i} \Rightarrow j>i
\end{aligned}
$$

From the revealed preference relations, described in the above proof, we can determine the technical efficiency upper bounds. This is possible by a simple modification of Theorem 8.2 in order to allow for technical progress.

Theorem 8.5: Suppose $D=\left\{\left(c_{i}, x^{i}, y_{i}\right)\right\}_{i \in I}$ is a finite data set. Let $R$ be the corresponding revealed preference relation for technical progress, determined by

$$
x^{j} R x^{i} \Leftrightarrow c_{i}\left(x^{j}\right) \leq c_{i}\left(x^{i}\right) \text { and } j \leq i \quad\left(i_{i} j \in I\right) .
$$

Then the technical efficiency upper bounds $t_{i}$ for producer demand with technical progress are grven as

$$
t_{i}=y_{i} / u_{i}, u_{i}=\max \left(y_{k} \mid k \in I: x^{k} R^{*} x^{i}\right\} \quad(i \in I)
$$

Proof: By Theorem 8,3 and the definition of demand $D_{d}^{f}$ for frontier production functions, the following conditions are equivalent:

(i) There exist a series of production functions $f f_{i} f_{i}$ such that $f_{j} \leq f_{i}$ if $j \leq i$, and $f_{i}\left(x^{i}\right)=u_{i},\left(c_{i}, x^{i}, y_{i}\right) \in D_{d}^{f}\left(f_{i}\right)$, for all $i \in I$.

(ii) There exist outputs $u_{i}$ such that

$$
\begin{array}{ll}
u_{i} \geq y_{i} \quad(i \in I), & \\
c_{i}\left(x^{j}\right) \leq c_{i}\left(x^{i}\right) \Rightarrow u_{j} \leq u_{i} & (i, j \in I: j \leq i), \\
c_{i}\left(x^{j}\right)<c_{i}\left(x^{i}\right) \Rightarrow u_{j}<u_{i} & (i, j \in I: j \leq i) .
\end{array}
$$

So, to maximize the technical efficiency $t_{i}$, we have to minimize $t_{i}$ in (ii). From Theorem 8.4 it follows that there exists always such a solution, satisfying above conditions in (i) and (ii). Now, using the approach in the proof of Theorem 8.2, we can show that such a solution can be found by 
solving the following problem:

$$
\text { inf } \left.u_{i} \quad u \in I\right)
$$

subject to

$$
\begin{aligned}
& u_{i} \geq y_{i} \quad(i \in I)_{i} \\
& u_{i} \leq u_{j}
\end{aligned} \quad\left(i, j \in I: x_{i} R x_{j}\right) .
$$

This problem has indeed the given solution

$$
u_{i}=\max \left\{y_{k} \mid k \in I: x^{k} R^{*} x^{i}\right\} \quad(i \in I) .
$$

Application of the above theorem to the Dutch data had the following result. There is only one year for which the technical efficiency upper bound is less than one. For this year, the year $t=1975$, we obtain $t_{i}=98.4 \%$.

\subsection{Hicks Neutral Change}

The popularity of Hicks neutral change is due to the fact that a simple transformation may convert data of producer demand with Hicks neutral change to data of producer demand behaviour without Hicks neutral change. This is the subject of the next theorem.

Theorem 8.6: Let $c: X \rightarrow \mathbb{R}_{+}$and $f: X \rightarrow \mathbb{R}$. Then one has

$$
(c, x, y) \in \mathcal{D}_{d}^{o}(t f) \Leftrightarrow\left(c, x, t^{-1} y\right) \in \mathcal{D}_{d}^{o}(f) \quad(t>0)
$$

Proof: Obvious.

Choosing the scale factor $t$ for Hicks neutral change means in fact that we may choose the resulting Hicks neutral changed output $t^{-1} y$. Hence, if we wish to test producer demand with. Hicks neutral change, we may as well test producer demand without observed output. This is stated in the following theorem.

Theorem 8.7: Suppose $D=\left\{\left(c_{i}, x^{i}\right)\right\}_{\text {iel }}$ is a data set, where $c_{i}: X \rightarrow \mathbb{R}_{+}$, and suppose $y_{i}>0$, for all $i \in I$. Then the following conditions are equivalent:

(1) There exist a function $f: X \rightarrow \mathbb{R}$ and numbers $t_{i}>0$, such that

$$
\left(c_{i}, x^{i}, y_{i}\right) \in \mathcal{D}_{d}^{0}\left(t_{i} f\right) \quad(i \in I) .
$$

(ii) $F_{d}(D) \neq \varnothing$.

Proof $(\mathrm{i}) \Rightarrow(\mathrm{ii}):$ By Theorem 8.6 one has

$$
\left(c_{i}, x^{i}, y_{i}\right) \in D_{d}^{o}\left(t_{i} f\right) \Leftrightarrow\left(c_{i}, x^{i}, t_{i}^{-1} y_{i}\right) \in D_{d}^{o}(f) \quad(i \in I)
$$

and we have thus $f \in \mathcal{F}_{d}(D)$.

(ii) $\Rightarrow$ (i): Let $f \in \mathcal{F}_{d}(D)$. We may suppose $f\left(x^{i}\right)>0$ for all $i \in I$. Otherwise we may apply a suitable monotonic transformation, because mof $\in \mathcal{F}_{d}(D)$ for any monotonic transformation $m: \mathbb{R} \rightarrow \mathbb{R}$. Now, for

$$
t_{i}=y_{i} / f\left(x^{i}\right) \quad(i \in I)
$$

one has

$$
\left(c_{i}, x^{i}, y_{i}\right) \in D_{d}^{o}\left(t_{i} f\right) \quad(i \in I)
$$

By the above theorem the assumption of Hicks neutral progress with observed outpul is equivalent to the assumption of producer demand without observed output. We may test the latter using of a revealed preference test. It appeared that the Dutch industry data without abserved output, were compatible with the hypothesis of producer demand. Hence, by Theorem 8.7 the whole data set - including observed output - is compatible with the hypothesis of Hicks neutral changing producer demand. 
Another approach to look at the above problem is to solve the restictions on the factors $t_{i}$ for Hicks neutral change. As is done in the following theorem.

Theorem 8.8: Suppose $D=\left\{\left(c_{i}, x^{i}, y_{i}\right)\right\}_{\text {ier }}$ is finte data set, there $y_{i}>0$ and $c_{i} * X \rightarrow \mathbb{R}_{+}$, for all $i \in I$. Then the following conditions are equivalent:

(i) There exist a function $f: X \rightarrow \mathbb{R}$ and numbers $t_{i}>0$ such that

$$
\left(c_{i}, x^{i}, y_{i}\right) \in \mathcal{D}_{d}^{o}\left(t_{i} f\right) \quad(i \in I) \text {. }
$$

(ii) There exist values $\phi_{i}=\ln t_{i}$ such that

$$
\phi_{i}-\phi_{j} \leq a_{i j}, \phi_{i}-\phi_{j}<b_{i j} \quad(i, j \in I),
$$

where

$$
\begin{aligned}
& a_{i j}= \begin{cases}\ln \left(y_{i} / y_{j}\right) & \left(i, j \in I: c_{i}\left(x^{j}\right) \leq c_{i}\left(x^{i}\right)\right), \\
\infty & (\text { otherwise }),\end{cases} \\
& b_{i j}= \begin{cases}\ln \left(y_{i} / y_{j}\right) & \left(i, j \in I: c_{i}\left(x^{j}\right)<c_{i}\left(x^{i}\right)\right), \\
\infty & \text { (otherwise). }\end{cases}
\end{aligned}
$$

Proof: By Theorem 8.6 one has

$$
\left(c_{i}, x^{i}, y_{i}\right) \in \mathcal{D}_{d}^{o}\left(t_{i} f\right) \Leftrightarrow\left(c_{i}, x^{i}, t_{i}^{-1} y_{i}\right) \in \mathcal{D}_{d}^{o}(f) \quad(i \in I)
$$

By Theorem 8.1 the latter is equivalent to

$$
\begin{array}{ll}
c_{i}\left(x^{j}\right) \leq c_{i}\left(x^{i}\right) \Rightarrow t_{j}^{-1} y_{j} \leq t_{j}^{-1} y_{i} & (i, j \in I), \\
c_{i}\left(x^{j}\right)<c_{i}\left(x^{i}\right) \Rightarrow t_{j}^{-1} y_{j}<t_{i j}^{-1} y_{i} & (i, j \in I) .
\end{array}
$$

Hence, we obtain that (i) is equivalent to the existence of numbers $t_{i}$ such that

$$
\begin{array}{ll}
t_{i} / t_{j}<y_{i} / y_{j} & \left(i, j \in I: c_{i}\left(x^{j}\right)<c_{i}\left(x^{i}\right)\right) \\
t_{i} / t_{j} \leq y_{i} / y_{j} & \left(i, j \in I: c_{i}\left(x^{j}\right) \leq c_{i}\left(x^{i}\right)\right) .
\end{array}
$$

By taking the logarithm on both sides of the above inequalities, this is equivalent to (ii).

Using the above theorem, we may compute lower and upper bounds for the levels $t_{i}$ of Hicks neutral change. These bounds follow from the inequalities

$$
\phi_{i}-\phi_{j} \leq a_{i j} \quad(i, j \in I)
$$

in Theorem 8.8 , using $\phi_{i}=\ln t_{i}$. The solution follows from the shortest path matrix $A^{*}$ of $A=\left[a_{i j}\right]$ and is given by

$$
\phi_{i}-\phi_{j} \leq a_{i j}^{*} \quad(i, j \in I) \text {. }
$$

The definition of a shortest path matrix is given in Appendix $C$. The idea of this matrix is as follows. When $a_{i j}, i, j \in I_{y}$ represents the cost of moving from to $j$ then $a_{i j}^{*}$ represents the cost of the cheapest path from to $j$.

Figure 8.1 displays the lower and upper bounds

$$
\mathrm{e}^{-a_{1 i}^{*}} \leq t_{i} / t_{1} \leq \mathrm{e}^{a_{i 1}^{*}} \quad(i, j \in I)
$$

with the first year as base year. In general one may derive these bounds from the output values $y_{i}$ and the revealled preference relation $R$ for unobserved output. One may prove that one has

$$
e^{a_{i j}^{*}}= \begin{cases}y_{i} / y_{j} & \left(i, j \in I: i R^{*} y\right)_{2} \\ \infty & \text { (otherwise). }\end{cases}
$$

For example, the Dutch industry data satisfies 


$$
c_{i}\left(x^{n}\right)<c_{i}\left(x^{n}\right) \quad(t \in I: \quad x \neq 1)
$$

which means

$$
\left\{R^{*} 1, \text { not } 1 R^{*} i \quad(i \in 1: i \neq 1)\right. \text {. }
$$

Hence, the lower and upper bounds that are displayed in Figure 8.1, are in this particular case equal to

$$
0=\mathrm{e}^{-a_{1 i}^{*}} \leq t_{i} / t_{1} \leq \mathrm{e}^{a_{i 1}^{*}}=y_{i} / y_{1} \quad(\dot{i} \in I: i \neq 1)
$$

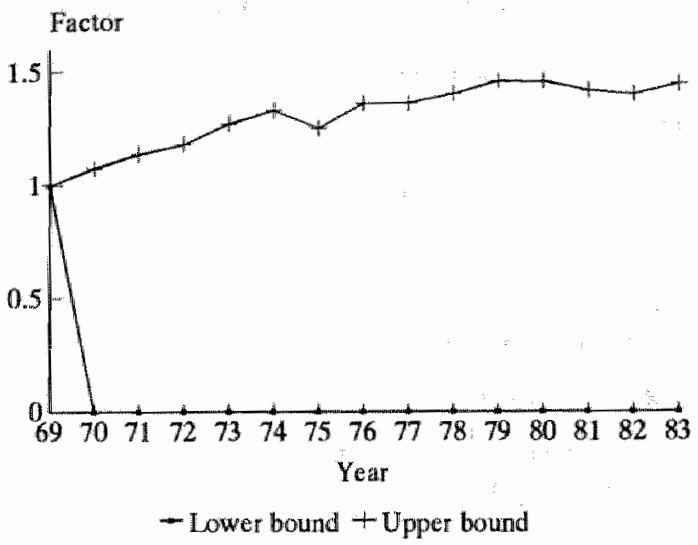

Figure 8.1. Bounds Hicks Neutral Change

Usually we assume that the change of the production function is due to progress. For Hicks neutral progress the following theorem is available.

Theorem 8.9: Suppose $D=\left\{\left(c_{i}, x^{i}, y_{i}\right)\right\}_{i \in I}$ is a finite data set, where $y_{i}>0$ and $c_{i}: X \rightarrow \mathbb{R}_{+}$, for all $i \in I$. Then the following conditions are equivalent:

(i) There exist a function $f: X \rightarrow \mathbb{R}$ and numbers $t_{i}>0$, such that $t_{j} \leq t_{i}$ if $j \leq i$, and

$$
\left(c_{i}, x^{i}, y_{i}\right) \in D_{d}^{a}\left(t_{i} f\right) \quad(t \in I) .
$$

(ii) There exist walues $\phi_{\mathrm{i}}=\ln t_{\mathrm{i}}$ such that

$$
\begin{aligned}
& \phi_{i}-\phi_{j} \leq \min \left\{a_{i j}, c_{i j}\right\} \\
& \phi_{i}-\phi_{j}<b_{i j}
\end{aligned} \quad(i, j \in I), \quad(i, j \in I),
$$

where

$$
\begin{aligned}
& a_{i j}= \begin{cases}\ln \left(y_{i} / y_{j}\right) & \left(i_{i} j \in I: c_{i}\left(x^{j}\right) \leq c_{i}\left(x^{i}\right),\right. \\
\infty & (\text { atherwise }),\end{cases} \\
& b_{i j}= \begin{cases}\ln \left(y_{i} / y_{j}\right) & \left(i, j \in I: c_{i}\left(x^{j}\right)<c_{i}\left(x^{i}\right)\right), \\
\infty & \text { (otherwise) }\end{cases}
\end{aligned}
$$




$$
c_{i j}= \begin{cases}a & (i, j \in I: i \leq j) \\ \infty & \text { (otherwise). }\end{cases}
$$

Proof: Analogous to the proof of theorem 8.8. We only have to include condition $t_{j} \leq t_{i}$ if $j \leq i$, which is equivalent to $t_{i} / t_{j} \leq 1$ if $i \leq j$. Taking the logarithm on both sides of the above inequality $t_{i} / t_{j} \leq 1$ yields the additional restrictions of $c_{i j}$ in (ii).

Condition (ii) in the above theorem is a shortest path problem that can be solved with Theorem C.3, given in Appendix C. When the theorem is applied to the Dutch industry data, it appears that one observation should be removed in order to obtain a data set, which is consistent with producer demand. Using bisection and Theorem C.3, we may determine the economical efficiency upper bound for the whole data set. The result, where again the efficiency transformation given in Example 2.3 is used, is $e=99.8 \%$.

For this efficiency level $e$, we may compute lower and upper bounds for the levels $t_{i}$ of Hicks neutral progress that belong to the transformed data $\Phi_{e} D$. These bounds follow from the inequalities

$$
\phi_{i}-\phi_{j} \leq d_{i j}=\min \left\{a_{i j}, c_{i j}\right\} \quad(i, j \in I)
$$

in Theorem 8.9 , where

$$
\phi_{i}=\ln t_{i} \quad(i \in I) \text {. }
$$

The solution follows from the shortest path matrix $D^{*}$ of $D=\left[d_{i j}\right]$ and is given by

$$
\phi_{i}-\phi_{j} \leq d_{i j}^{*} \quad(i, j \in I)
$$

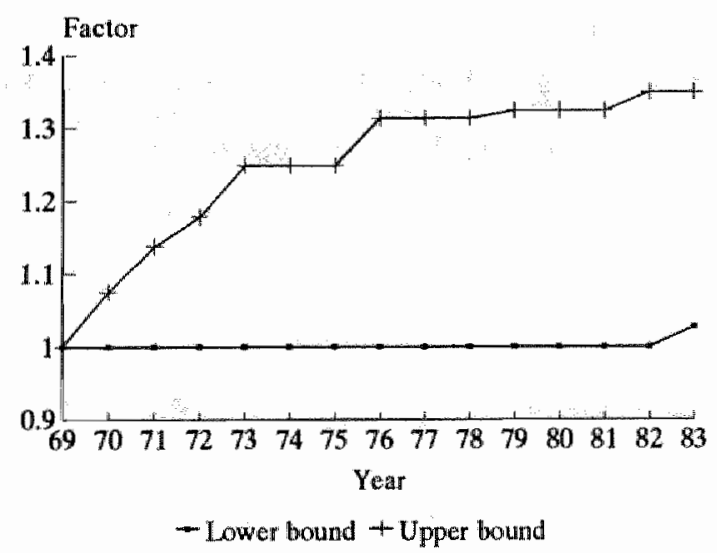

Figure 8.2 Bounds Hicks Nentral Progress

Figure 8.2 displays the lower and upper bounds

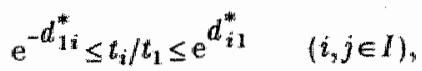

with the first year as base year. Since there is progress, the lower bound is larger or equal to one. It appears that the lower bound is equal to one, 
except for the last year. For the upper bound, however, we get nontrivial information.

\subsection{Neutral Change}

Neutral change is similar to Hicks neutral change, but now an input vector is scaled instead of the produced output. The following theorem is similar to Theorem 8.6, which considered Hicks neutral change.

Theorem 8.10: Suppose o: $X_{1} \times X_{2} \rightarrow \mathbb{R}_{4}$ and $f: X_{1} \times X_{2} \rightarrow \mathbb{R}$ Then for every $t>0$ with $\left[x_{1}, t x_{2}\right] \in X_{1} \times X_{2}$, one has

$$
\left(c, x^{*}, y\right) \in D_{d}^{o}(f) \Leftrightarrow\left(c_{t}\left[x_{1}^{\prime}, t x_{2}^{\prime}\right], y\right) \in D_{d}^{o}(f)
$$

where

$$
f_{t}\left(x_{1}, x_{2}\right)=f\left(x_{1}, t x_{2}\right), c_{t}\left(x_{1}, x_{2}\right)=c\left(x_{1}, t^{-1} x_{2}\right) \quad\left(x_{1} \in X_{1}, x_{2} \in X_{2}\right)
$$

Proof: Using the transformation

$$
v_{1}=x_{1}, v_{2}=t x_{2} \quad\left(x_{1} \in X_{11}, x_{2} \in X_{2}\right)
$$

we obtain

$$
\begin{aligned}
& c(x) \leq c\left(x^{2}\right) \Leftrightarrow c_{t}(v) \leq c_{t}\left(x^{t}\right) \\
& f_{1}(x) \leq f_{d}\left(x^{n}\right) \Leftrightarrow f(v) \leq f\left(x^{t}\right) .
\end{aligned}
$$

Hence the result follows directly from the definition of $\mathcal{D}_{d}^{\circ}$.

The idea to solve the restrictions on producer demand for neutral change is simple. With the above theorem we can convert the data to data, to which the usual nomparametric restrictions for producer demand can be applied. This approach is used in the following theorem.

Theorem 8.11: Suppose $D=\left\{\left(c_{i}, x^{i}, y_{i}\right)\right\}_{\text {ieI }}$ is a finite data set in which all functions $c_{n}: X_{1} \times X_{2} \rightarrow \mathbb{R}_{+}$are of the form

$$
c_{i}(x)=v_{i}\left(x_{1}\right)+w_{i}\left(x_{2}\right) \quad\left(i \in I, x \in X_{1} \times X_{2}\right),
$$

where $w_{i}: X_{2} \rightarrow \mathbb{R}_{+}$is linearly homogeneous. Then the following conditions are equivalent:

(i) There exist a function $f: X_{1} \times X_{2} \rightarrow \mathbb{R}$ and numbers $t_{i}>0$, such that for

$$
f_{i}(x)=f\left(x_{1}, t_{i} x_{2}\right) \quad\left(i \in I, x \in X_{1} \times X_{2}\right)
$$

one has

$$
\left(c_{i}, x^{i}, y_{i}\right) \in D_{d}^{a}\left(f_{i}\right) \quad(i \in I) \text {. }
$$

(ii) There exwst walues $\phi_{i}=$ lin $t_{i}$ such that

$$
\phi_{i}-\phi_{j} \leq a_{i j}, \phi_{i}-\phi_{j}<b_{i j} \quad(i, j \in I),
$$

where

$$
\begin{aligned}
& a_{i j}= \begin{cases}\ln \left(w_{i}\left(x_{2}^{j}\right)\left[c_{i}\left(x^{i}\right)-v_{i}\left(x_{1}^{j}\right)\right]^{-1}\right) & \left(i, j \in I: y_{j} \geq y_{i}, c_{i}\left(x^{i}\right)>v_{i}\left(x_{1}^{j}\right),\right. \\
\infty & \text { (otherwise) },\end{cases} \\
& b_{i j}= \begin{cases}\ln \left(w_{i}\left(x_{2}^{j}\right)\left[c_{i}\left(x^{i}\right)-v_{i}\left(x_{1}^{j}\right)\right]^{-1}\right) & \left(i_{i}, j \in I: y_{j}>y_{i}, c_{i}\left(x^{i}\right)>v_{i}\left(x_{1}^{j}\right)\right), \\
\infty & \text { (otherwise). }\end{cases}
\end{aligned}
$$

Proof: From Theorem 8.1 and Theorem 8.10 we obtain that condition (i) is equivalent to the existence of numbers $t_{i}$ such that

$$
\begin{array}{ll}
c_{i}\left(x_{1}^{j}, t_{i}^{-1} t_{j} x_{2}^{j}\right)>c_{i}\left(x^{i}\right) & \left(i, j \in I: y_{j}>y_{i}\right), \\
c_{i}\left(x_{1}^{j}, t_{i}^{-1} t_{j} x_{2}^{j}\right) \geq c_{i}\left(x^{i}\right) & \left(i, j \in I: y_{j} \geq y_{i}\right) .
\end{array}
$$


Because we have assumed that we can writie

$$
c_{i}\left(x_{1}^{j}, t_{i}^{\infty 1} t_{j} x_{2}^{j}\right)=v_{i}\left(x_{1}^{j}\right)+t_{i}^{-1} t_{j} w_{i}\left(x_{2}^{j}\right) \quad(i, j \in I)
$$

this condition can be rewritten as

$$
\begin{array}{ll}
t_{i}^{-1} t_{j} w_{i}\left(x_{2}\right)>c_{i}\left(x^{i}\right)-v_{i}\left(x_{1}^{j}\right) & \left(i, j \in I: y_{j}>y_{i}\right), \\
t_{i}^{-1} t_{j} w_{i}\left(x_{2}^{j}\right) \geq c_{i}\left(x^{i}\right)-v_{i}\left(x_{1}^{j}\right) & \left(i, j \in I: y_{j} \geq y_{i}\right) .
\end{array}
$$

Because the left side of the above inequalities is nonnegative, we may drop all inequalities that are not positive at the right side. Finally, we obtain (ii) by taking the logarithm at both sides of the remaining inequalities.

We may test condition (iii) using Theorem $C .3$, given in Appendix $C_{n}$ Note that the special additive form $c_{i}=v_{i}+w_{i}$ of the cost functions is necessary in the above theorem. It is used to derive the restrictions on $t_{i}^{-1} t_{j}$.

In case of neutral progress there is the additional restriction that the coefficients $t_{i}$ for neutral change are increasing. This additional restriction leads to the following theoren, which is a simple variation of the previous theorem.

Theorem 8.12: The following conditions are equivalent:

(i) There exist $t_{i}>0$ satisfying Theorem 8.11 (i), such that $t_{j} \leq t_{i}$ if $j \leq i$.

(ii) There exist values $\phi_{i}=\ln t_{i}$ such that

$$
\begin{aligned}
& \phi_{i}-\phi_{j} \leq \min \left\{a_{i j,}, c_{i j}\right\} \\
& \phi_{i}-\phi_{j}<b_{i j} \quad(i, j \in I),
\end{aligned} \quad(i, j \in I),
$$

where $a_{i j}$ and $b_{i j}$ are given in Theorem 8.11 and

$$
c_{i j}= \begin{cases}0 & (i, j \in I: i \leq j), \\ \infty & (\text { atherwise }) .\end{cases}
$$

Proof: Add the restriction $t_{j} \leq t_{i}$ if $j \leq i$, to the result described in Theorem 8.11.

A test for the existence of numbers $\phi_{i}$ satisfying condition (ii) in the above theorem is given in Appendix $\mathrm{C}$ in Theorem C.3. I applied condition (ii) of Theorem 8.12 to two different inputs of the Dutch industry data: labour and capital. Using this theorem, we may determine the largest possible data set that is consistent with neutral progressing producer demand. The result was as follows. For both neutral progress of labour and capital one observation has to be deleted to get: a consistent data set.

Another application of Theorem 8.12 , withotut removing observations from the data, is the computation of an economical effictency upper bound. Such at bound represents a violation measure for the hypothesis of neutral progressing producer demand. To compute an efficiency upper bound, there are certain restrictions that the used efficiency transfomation has to satisfy. In Theorem 8.11 and 8.12, it is assumed that we may write the cost functions in the form

$$
c(x)=v\left(x_{1}\right)+w\left(x_{2}\right) \quad\left(x \in X_{1} \times X_{2}\right)
$$

where $w$ is a linearly homogeneous function. This assumption may cause problems. If data satisfy this assumption, this is not necessarily the case for the transformed data, for instance, when we use the efficiency transformations $\Phi_{e}$ or $A_{e}$ given in Example 2.3 and 3.1 respectively. However, we may prove both theorems, using the relaxed assumption that we may write

$$
c_{i}\left(x_{1}^{j}, \lambda x_{1}^{j}\right)=w_{i}\left(x_{1}^{j}\right)+w_{i}\left(\lambda x_{2}^{j}\right) \quad(\lambda>0, i, j \in I: i \neq j),
$$

in which $w_{i}\left(\lambda x_{2}^{j}\right)$ is nonnegative and linearly homogeneous in $\lambda$. For empirical 
data we may expect that this property remains satisfied, after transformation by the efficiency transformation $\Phi_{e}$ or $A_{e}$. So in practice there is no problem at all. However, if we wish to apply the above theorems to any possible data, we might choose the following suitable efficiency transformation, which leaves the desired property intact.

Example 8.1 Suppose $\left(c_{;}\right)$is a data observation such that

$$
c(x)=v\left(x_{1}\right)+w\left(x_{2}\right) \quad\left(x \in X=X_{1} \times X_{2}\right),
$$

where $w$ is linearly homogeneous. Using the efficiency transformation $\phi_{e}$ and $A_{e}$, given in Example 2.3 and Example 3.1 respectively, we define the functions $v^{e}$ and $w^{e}$ as follows

$$
\left(w^{e}, x_{1}^{\prime}\right)=\Phi_{e}\left(v, x_{1}^{\prime}\right),\left(w^{e}, x_{2}^{\prime}\right)=\Lambda_{e}\left(w ; x_{2}^{\prime}\right)
$$

Then $\hat{\Phi}_{e}\left(c, x^{\prime}\right)=\left(\hat{c}{ }^{e}, x^{\prime}\right)$, where

$$
\hat{c}^{e}(x)=y^{e}\left(x_{1}\right)+w^{e}\left(x_{2}\right) \quad\left(x \in X_{1} \times X_{2}\right)
$$

defines an efficiency transformation, where a linearly homogeneous function w is transformed into a linearly homogeneous function we.

In the case of the Dutch industry data it was not necessary to use the above efficiency transformation. It was allowed to use the efficiency

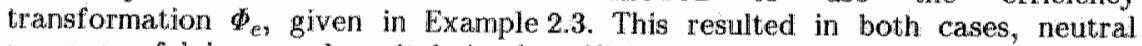
progress of labour and capital, in the efficiency upper bound $e=99.8 \%$.

Using this efficiency level, I computed the lower and upper bounds for the scale factors of neutrall progress that belong to the transformed data $\Phi_{e} D$. The resulting bounds are

$$
\mathrm{e}^{-d_{j i}^{*}} \leq t_{i} / t_{j} \leq \mathrm{e}^{d_{i j}^{*}} \quad(i, j \in I)
$$

where we obtain

$$
d_{i j}=\min \left\{a_{i j}, c_{i j}\right\} \quad(i, j \in I)
$$

from Theorem 8.12 . I computed these bounds for $t_{i} / t_{j}$ for a fixed base year $j$, the first observation year. For both cases, capital and labour, the result was similar and not very informative. The lower bounds were equal to one, except for a value close to one for the last observation year, and the upper bounds equal to infinity. It seems that the specification of the production function is too wide for the derivation of narrow nonparametric restrictions. Later, when we suppose that the production function is linearly homogeneous, the results will be more promising. The reason why the results are a bit disappointing in this case is the following. We do not obtain any information, when the data have the following properties:

1. the data are consistent with the assumption of producer demand;

2. output is increasing over time.

This result is stated in the theorem below.

Theorem 8.13: Suppose $D=\left\{\left(c_{i}, x^{i}, y_{i}\right)\right\}_{i \in I}$ is a finte data set in which all functions $c_{i}: X_{1} \times X_{2} \rightarrow \mathbb{R}_{+}$are of the form

$$
c_{i}(x)=v_{i n}\left(x_{1}\right)+w_{i}\left(x_{2}\right) \quad\left(i \in I, x \in X_{1} \times X_{2}\right),
$$

where $w_{i}: X_{3} \rightarrow \mathbb{R}_{+}$is limearly homogeneous. Suppose $\mathcal{F}_{d}^{o}(D) \neq \emptyset$ and output strictly increasing, i.e. $y_{j}>y_{i}$ if $j>i$. Let $t_{i}>0, \quad \in \in I$, be an ancreasing series of numbers. Then there exists a function $f: X_{1} \times X_{2} \rightarrow \mathbb{R}$ such that every function

$$
f_{i}(x)=f\left(x_{1}, t_{i} x_{2}\right) \quad\left(i \in I, x \in X_{1} \times X_{2}\right)
$$

satisfies $\left(c_{i}, x^{i}, y_{i}\right) \in \mathcal{D}_{d}^{a}\left(f_{i}\right)$. 
Proof: Let $f \in \mathcal{F}_{d}(D)$. Then by Theorem 8.1, we have

$$
\begin{array}{ll}
y_{j}>y_{i} \Rightarrow c_{i}\left(x^{j}\right)>c_{i}\left(x^{i}\right) & (i, j \in I), \\
y_{j} \geq y_{i} \Rightarrow c_{i}\left(x^{j}\right) \geq c_{i}\left(x^{i}\right) & (i, j \in I) .
\end{array}
$$

Now, for an increasing series $t_{i}$, $i \in I$, we have

$$
c_{i}\left(x_{1}^{j}, t_{j} t_{i}^{-1} x_{2}^{j}\right) \geq c_{i}\left(x_{1}^{j}, x_{2}^{j}\right) \quad(i, j \in I: j \geq i)
$$

Hence, the assumptionis imply

$$
\begin{array}{ll}
y_{j}>y_{i} \Rightarrow c_{i}\left(x_{1}^{j}, t_{i}^{-1} t_{j} x_{2}^{j}\right) \geq c_{i l}\left(x^{j}\right)>c_{i}\left(x^{i}\right) & (i, j \in I), \\
y_{j} \geq y_{i} \Rightarrow c_{i}\left(x_{1}^{j}, t_{i}^{-1} t_{j} x_{2}^{j}\right) \geq c_{i i}\left(x^{j}\right) \geq c_{i}\left(x^{i}\right) & (i, j \in I) .
\end{array}
$$

Thus by Theorem 8.1 there exists a function $f$ such that

$$
\left(c_{i}^{t},\left[x_{1}^{i}, t_{i} x_{2}^{i}\right], y_{i}\right) \in D_{d}^{o}(f) \quad(i \in I)
$$

where

$$
c_{i}^{*}\left(x_{1}, x_{2}\right)=c_{i}\left(x_{1}, t_{i}^{-1} x_{2}\right) \quad\left(x_{1} \in X_{1}, x_{2} \in X_{2}, i \in I\right),
$$

which is by Theorem 8.10 equivalent to $\left(c_{i}, x^{i} ; y_{i}\right) \in \mathcal{D}_{d}^{o}\left(f_{i}\right)$ for all $i \in I$.

So we may conclude that data with increasing output, which are nearly consistent with producer demand, tend to hide information about neutral technical progress. It is a pity that empirical production data often have these properties.

\subsection{Efficiency Transformations for Neutral Change}

To finish this chapter I will consider the efficiency transformation that is mentioned in the previous section. It still remains to answer how we can compare the results for efficiency transformation $\hat{\Phi}_{e}$, given in the previous section, with results for the standard efficiency transformation $\phi_{e,}$ given in Example 2.3. At the end of this section I will show that both efficiency transformations may generate the same results.

First let us look at some properties of both efficiency transformations. Write

$$
\Phi_{e}\left(c, x^{\prime}\right)=\left(c^{e}, x^{\prime}\right), \hat{\Phi}_{e}\left(c, x^{\prime}\right)=\left(\hat{c}{ }^{e}, x^{\prime}\right),
$$

and assume $c=v+w$ with $w$ linearly homogeneous. By the definition of both efficiency transformations, we have $\hat{c}^{e} \leq c^{e}$. So we can derive inmediately the following general result.

Theorem 8.14: Suppose $D$ is data set. Let $\hat{\Phi}_{e}$ and $\phi_{e}$ be the efficiency transformations given in Example 8.1 and 2.3 respectively. Then:

(a) $\mathcal{F}_{d}\left(\hat{\Phi}_{e} D\right) \subset \mathcal{F}_{d}\left(\hat{\Phi}_{e} D\right)$.

(b) $\hat{e}_{d}(F, D) \leq e_{d}(F, D)$, where $\hat{e}_{d}$ and $e_{d}$ are the efficiency upper bounds for the efficiency transformations $\hat{\Phi}_{e}$ and $\phi_{\dot{c}}$ respectioely, and $F$ is an arbitrary given set of functions.

Furthermore, the above is alsa valid if output is observed and in case frontier production functions are considered.

Proof: (a) Use the definition of $\mathcal{F}_{d}$ and the inequality $\hat{c}^{c} \leq c^{r}$.

(b) Follows immediately from (a).

It may happen that both efficjency measures lead to the same results. The following theorem presents an example of such a case.

Theorem 8.15: Suppose $D=\left\{\left(c_{i}, x^{i}\right)_{i \Subset I} \dot{x}_{\mathrm{s}}\right.$ a data set. Let $\dot{\Phi}_{\mathrm{e}}$ and $\Phi_{e}$ be the efficiency transformations given in Example 8.1 and 2.3 respectively. Suppose 


$$
x^{j} \neq\left(x_{1}, \lambda x_{2}\right) \quad(i, j \in I, \lambda \in \mathbb{R}: \lambda \neq 1)
$$

Then:

(a) The revealed preference conditions for the data sets $\Phi_{e} D$ and $\Phi_{e} D$ are equal to each other.

(b) Let $F$ be the set of all functions $f: X \rightarrow \mathbb{R}$. Then $\hat{e}_{d}(F, D)=e_{d}(F, D)$, where $\hat{e}_{d}$ and $e_{u d}$ are the efficiency upper bounds for the efficiency transformations $\hat{\Phi}_{e}$ and $t_{e}$ respectively.

Furthernore, the above is also valid if output is abserved or frontier production functions are considered.

Proof: (a) Using the same notation as usual, we obtain for the transformed cost functions the equality $\hat{k}_{i}^{e}\left(x^{j}\right)=c_{i}^{e}\left(x^{j}\right)$ for all $i, j \in I$. Hence the revealed preference conditions, which depend only on these values, are for both datia sets equal to each other.

(b) There exists a rationalizing function if and only if the revealed preference conditions for the data are satisfied. So, (a) implies that both $\mathcal{F}_{d}\left(\hat{\Phi}_{e} D\right)$ and $\mathcal{F}_{d}\left(\Phi_{e} D\right)$ are together empty or nonempty. Hence, the result follows from the definition of $e_{d}$ as the supremum over efficiency levels $e$ for which a rationalizing function exists.

Now, let us consider the necessary and sufficient conditions for the existence of a production function in case output is observed or frontier production functions are considered. In that case only the revealed preference conditions and the observed outputs are of importance. Thus the necessary and sufficient conditions are the same for both efficiency transformations.

For empirical datan the condition in the above theorem are usually satisfied. So, it is in practice not important, which one of the efficiency measures is chosen. 


\section{LINEARLY HOMOGENEOUS PRODUCER DEMAND}

\subsection{Introduction}

In this chapter we shall consider producer demand when both the production function and cost functions are linearly homogeneous. In this case there is no need to restrict the theory to finite data sets. The following approach may simplify the search for conditions considering linearly homogeneous producer demand. This approach relies on the invariance of the hypothesis of linearly homogeneous producer demand under the scaling of observations, as is described in Theorem 3.1. Assuming output positive, one may replace the observations $\left\{\left(c_{i}, x^{i}, y_{i}\right)\right\}_{i \in I}$ by the observations $\left\{\left(c_{i}, y_{i}^{-1} x^{i}, 1\right)\right\}_{\text {ier }}$, scalled on the unit isoquant, which contain the same information as the original data. The advantage of using data that are scaled to an isoquant, is that we may weaken our hypothesis. As will be shown below, such data are not able to distinguish between the hypothesis of rational linearly homogeneous demand and the hypothesis of rational demand.

So, if we wish to test the hypothesis of rational linearly homogeneous demand, we may as well scale the data to an isoquant and test the resulting datia without the condition that the production function is linearly homogeneous. Why is this the case? If there exists a rationalizing function for the scaled datia, then - under very weak conditions - we may construct a linearly homogeneous function that shares the observed isoquant and which is compatible with the nonscaled data. The construction of such a function is as follows.

Suppose that there is a production function $f: X \rightarrow \mathbb{R}$, that passes the producer demand test for the scaled data. In that case we may use the surface

$$
S=\{x \in X \mid f(x)=1\}
$$

to construct a linearly homogeneous function $g: X \rightarrow R$, which passes also the producer demand test. This construction is obviously the following

$$
g(\lambda s)=\lambda \quad(s \in S, \lambda \geq 0) .
$$

Under weak conditions both functions $f$ and $g$ will display the same economical behaviour on the shared unit-isoquanh. So, for the scaled data, the existence of a rational production function $f$ in general, is in such a case equivalent to the existence of a linearly homogeneous rational production function $g$.

Remark: There are a few small theoretical problems in connection with the above approach, which are related to the mentioned weak conditions that have to be satisfied. They only are treated to complete the story. These minor problems, which we shall not encounter later on, are the following. Pirst, we have to prove that both $f$ and $g$ have the same economical behaviour on the shared unit-isoquant $S$. This is the case when the wealk condition

$$
f(x)<1 \Leftrightarrow g(x)<1 \quad(x \in X)
$$

is satisfied. Secondly, to obtain a well-defined linearly homogeneous function $g$, every ray from the origin through $S$,

$$
\{A s \mid \lambda \geq 0\} \quad(s \in S)
$$

has to cut the surface $S$ only once. Third, when these rays through $S$ do not cover $X$, i.e. 


$$
X \neq\{\lambda s \mid \lambda \geq 0, s \in S\}
$$

we have to extend the definition of $g$ on $X$, for example, by defining $g$ equal to zero for the remaining undefined values.

What can we conclude from the above in relation to the derivation of a theory. concerning linearly homogeneous producer behaviour? First, scale the data to the unit-isoquant. Then apply the theory that is derived for the case where there is no restriction on the production function. This yields necessary conditions for the existence of a Inearly homogeneous production function for given producer demand data. As argued above, such a necessary condition will often turn out to be a sufficient condition for linearly homogeneous producer demand.

To prove the existence of a linearly homogeneous production function, which may generate the given data, we can often derive such a function as the least upper bound over all possible linearly homogeneous production functions for the data set. Later on in this chapter, I will use such an approach to derive results. First, I derive conditions for linearly homogeneous demand using the theory given in Chapter 8.

\subsection{Conditions for Linearly Homogeneous Demand}

In this section we scale the data $\left\{\left(c_{i}, x^{i}, y_{i}\right)\right\}_{i \in I}$ to the unit-isoquant in order to derive necessary and sufficient conditions for the hypothesis of linearly homogeneous demand. We shall apply to these data the theory given in Chapter 8 , concerning the case where there is no restriction on the production function. By Theorem 8.1. the scaled data $\left\{\left(c_{i}, y_{i}^{-1} x^{i}, 1\right)\right\}_{\text {ief }}$ satisfy the hypothesis of producer demand, if one has

$$
\begin{array}{ll}
c_{i}\left(y_{j}^{-1} x^{j}\right) \leq c_{i}\left(y_{i}^{-1} x^{i}\right) \Rightarrow 1 \leq 1 & (i, j \in I), \\
c_{i}\left(y_{j}^{-1} x^{j}\right)<c_{i}\left(y_{i}^{-1} x^{i}\right) \Rightarrow 1<1 & (i, j \in I) .
\end{array}
$$

The first condition has no meaning, because it is always true. The second condition can be rewritten as

$$
c_{i}\left(y_{j}^{-1} x^{j}\right) \geq c_{i}\left(y_{i}^{-1} x^{i}\right) \quad(i, j \in I) .
$$

This condition is indeed a necessary and sufficient condition for the hypothesis of linearly homogeneous producer demand as is shown in the proof of the following theorem. A comparable theorem is stated in Hanoch and Rothschildt (1972) and Varian (1984).

Theorem 9.1: Swppose $D=\left\{\left(c_{i}, x^{i}, y_{i}\right)\right\}_{i \in I}$ is a data set, where $c_{i}: X \rightarrow \mathbb{R}_{+}$are linearly homogeneous, $c_{i}\left(x^{i}\right)>0$ and $y_{i}>0$, for all $i \in I$, and $X$ is a cone. Put

$$
F=\{f \in \mathcal{F}(D) \| f \text { linearly homogeneous }\} \text {. }
$$

Then the following conditions are equivalent:

(i) $F \neq 0$.

(ii) $y_{j} \leq y_{i} c_{i}\left(x^{j}\right) / c_{i}\left(x^{i}\right) \quad(i, j \in I)$

Proof: (i) (ii): Suppose $F \neq 0$. Then we obtain, as is described above this theorem, the inequalities

$$
c_{i}\left(y_{j}^{-1} x^{j}\right) \geq c_{i}\left(y_{i}^{-1} x^{i}\right) \quad(i, j \in I) \text {. }
$$

Hence, since all cost functions $c_{j}$ are linearly homogeneous, we have

$$
y_{j} \leq y_{i} c_{i}\left(x^{j}\right) / c_{i}\left(x^{i}\right) \quad(i, j \in I) .
$$

(ii) $\Rightarrow$ (i): Put

$$
f(x)=\inf _{i \in l} y_{i} c_{i}(x) / c_{i}\left(x^{i}\right) \quad(x \in X) .
$$


Then (ii) implies that we have $f\left(x^{i}\right)=y_{i}$ for all $i \in I$. This means $f \in F$, because $f$ is clearly linearly homogeneous as the infimum over linearly homogeneous functions.

I tested whether the Dutch industry data satisfied condition (ii) of the above theorem. It turned out that 13 observations have to be deleted, So, a subset of only two observations is consistent with the hypothesis of linearly homogeneous producer demand.

Using the above theorem, we can determine the economical efficiency upper bound for the efficiency transformation $\lambda_{e}$ given in Example 3.1. Application of Theorem 9.1 to the Dutch industry data, and using bisection, resulted in the efficiency level upper bound $e=89.6 \%$. The result is not very high, as we could have expected from the results in the previous section. This low efficiency level may be due to the fact that there is technical progress. As we shall see later on in this chapter, the allowance of technical progress. results in a less restrictive test with higher efficiency levels.

\subsection{Upper Bounds and Producer Demand}

In the previous section we have derived a necessary and sufficient condition for the existence of a linearly homogeneous production function. "To prove the existence of such a function, I used the following function

$$
f(x)=\inf _{i \in I} y_{i} c_{i}(x) / c_{i}\left(x^{i}\right) \quad(x \in X) .
$$

In this section I will show that the choice of this function was not an arbitrary choice. The chosen function is the least upper bound of all rationalizing linearly homogeneous functions. That this bound is used to prove the existence of a rationalizing linearly homogeneous function is not a coincidence. Theorem 3.13 states that a least upper bound over rationalizing linearly homogeneous functions is a rationalizing linearly homogeneous function ütself.

Let us recall the upper bound we have used for linearly homogeneous consumer behaviour. For a collection $F$ of functions $f: X \rightarrow \mathbb{R}$, the relative upper bound $U$, is equal to

$$
U_{f}\left(F, x, x^{\prime}\right) \equiv \sup _{f \in F} f(x) / f\left(x^{\prime}\right) \quad\left(x, x^{\prime} \in X\right) .
$$

In this chapter the following definition will be useful.

Definition: The upper borud $U$ is equal to

$$
U(F, x) \equiv \sup _{f \in F} f(x) \quad(x \in X)
$$

First I consider the upper bound for the simple case of one data ellement

Theorem 9.2: Suppose $c: X \rightarrow \mathbb{R}_{+}$is linearly homogeneous, $y>0, x^{4} \in X, c\left(x^{*}\right)>0$, and $X$ is a cone. Put

$$
F=\left\{f: X \rightarrow \mathbb{R} \mid f \in \mathcal{F}_{d}^{o}\left(\left\{\left(c, x^{*}, y\right)\right\}\right) \text { is linearly homogeneous }\right\} \text {. }
$$

Then $F \neq 0$ and:

(a) $U(F,) \in F$.

(b) One has

$$
y_{N}\left(f, x, x^{-1}\right)=c(x) / c\left(x^{*}\right)=y^{-1} U(F, x) \quad(x \in X) .
$$

Proof: (a) Put

$$
f(x)=y c(x) / c\left(x^{\prime}\right) \quad(x \in X)
$$

Then clearly $f \in F$ and thus we have only to prove (b). 
$(b)$ : For $f \in F$, as defined above in the proof of (a), we have

$$
c(x) / c\left(x^{\prime}\right)=f(x) / f\left(x^{\prime}\right) \leq U,\left(F, x, x^{\prime}\right) \quad(x \in X) \text {. }
$$

Moreover, by Theorem 3.10 one has

$$
f(x) / f(x) \leq c(x) / c\left(x^{\prime}\right) \quad(x \in X, f \in F) .
$$

Hence $U_{f}(F, x, x) \leq c(x) / c\left(x^{\prime}\right)$, which implies the desired equality

$$
U_{/}\left(F_{,}, x, x^{\prime}\right)=c(x) / c\left(x^{\prime \prime}\right) \quad(x \in X) .
$$

Moreover, by scaling the data we obtain $f\left(y^{-1} x^{x}\right)=y^{-1} f\left(x^{\prime}\right)=1$ for any $f \in F$, so that we have also

$$
U(F, x)=U /\left(F, x, y^{-1} x^{x}\right)=c(x) / c\left(y^{-1} x^{\prime}\right)=y c(x) / c\left(x^{i}\right) \quad(x \in X)
$$

The following theorem shows that the least upper bound of the family of linearly homogeneous production functions for a given data set is a member of this family. The theorem extends the results of the above theorem, which was concerned with only one data element, and the upper bound is now constructed for a general data set.

Theorem 9.3: Suppose $D=\left\{\left(c_{i}, x^{i}, y_{i}\right)\right\}_{i \in I}$ is a data set, where $c_{i}: X \rightarrow \mathbb{R}_{+}$are linearly homogeneous, $c_{i}\left(x^{i}\right)>0$ and $y_{i}>0$, for all $z \in I$, and $X$ is a cone. Put

$$
F=\left\{f \in \mathcal{F}_{d}^{\circ}(D) \mid f \text { lincarly komogeneous }\right\} \text {, }
$$

If $F \neq \emptyset$ then $U(F,) \in F$ and

$$
U(F, x)=\inf _{i \in I} y_{i} c_{i}(x) / c_{i}\left(x^{i}\right) \quad(x \in X) .
$$

Proof: Put

$$
f(x)=\inf _{i \in I} y_{i} c_{i}(x) / c_{i}\left(x^{i}\right) \quad(x \in X) .
$$

From the proof of Theorem 9.1 , we obtain $f \in F$. Hence, we have $f(x) \leq U(F, x)$, and it remains to prove the reverse inequality.

Theorem 9.2 states that we have for

$$
F_{i}=\left\{g \in \mathcal{F}_{d}^{a}\left(\left\{\left(c_{i}, x^{i}, y_{i}\right)\right\}\right) \mid g \text { linearly homogeneous }\right\} \quad(i \in I),
$$

the equality

$$
V\left(F_{i}, x\right)=y_{i} c_{i}(x) / c_{i}\left(x^{i}\right) \quad(i \in I, x \in X) .
$$

Because clearly $F \subset F$, we obtain thus

$$
U(F, x) \leq \inf _{i \in I} U\left(F_{i}, x\right)=\inf _{i \in I} y_{i} c_{i}(x) / c_{i}\left(x^{i}\right)=f(x) \quad(x \in X)
$$

\subsection{Frontier Production Functions}

The solution I will present for linearly homogeneous frontier production functions, has a resemblance to the solution for the case where there was no restriction on the production function. This case was treated in Section 8.3, where the infimum over rationalizing frontier production functions is determined. This infimum was not always attainable. In this section we shall find a minimal rationalizing frontier production function that attains the infimum. The following theorem states that such a production function always exists, assuming that there is at least one rationalizing production function for the data without observed outputs.

Theorem 9.4: Suppose $D=\left\{\left(c_{i}, x^{i}, y_{i}\right)\right\}_{i \in I}$ is a finite diota set, where $c_{i}: X \rightarrow \mathbb{R}_{+}$ are linearly homogeneons, $c_{i}\left(x^{i}\right)>0$ and $y_{i}>0$, for all $i \in I$, and $X$ is a cone. Suppose $F \neq 0$ and put 


$$
\begin{aligned}
& F^{*}=\left\{f \in \mathcal{F}_{d}(D) \mid f \text { linearly homogeneous }\right\} \\
& \left.F^{f}=\| f \in \mathcal{F}_{d}^{f}(D) \mid f \text { linearly homogeneous }\right\}, \\
& l_{i}=\max _{k \in I} y_{k} L_{j}\left(F, x_{i}^{i} x^{k}\right) \quad(i \in I), \\
& l(x)=\min _{i \in I} l_{i} c_{i}(x) / c_{i}\left(x^{i}\right) \quad(x \in X) .
\end{aligned}
$$

Then $l \in F^{*}$ and $L\left(F^{f}, x^{i}\right)=l\left(x^{i}\right)=l_{i}$ for all $i \in I$.

Proof: Theorem 3.4 and $c_{i}\left(x^{i}\right)>0$ imply that we have $f\left(x^{i}\right)>0$ if $f \in F$. By definition of $F$ and $F$ we have

$$
\begin{aligned}
& f\left(x^{k}\right) \geq y_{k} \quad\left(f \in F^{f}, k \in I\right), \\
& f\left(x^{i}\right) / f\left(x^{k}\right) \geq L_{\gamma}\left(F, x^{k}, x^{k}\right) \quad(i, k \in I, f \in F) .
\end{aligned}
$$

Using $F^{f} \subset F$ we obtain thus

$$
\left.f\left(x^{i}\right) \geq y_{k} f\left(x^{i}\right) / f\left(x^{k}\right) \geq y_{k} L_{/}\left(F, x^{i}, x^{k}\right) \quad \forall \in F^{f}, i, k \in I\right) .
$$

So, the definition of $L$ implies $L\left(F^{f}, x^{i}\right) \geq l_{i}$ for all $i \in I$. To complete the proof, we have to show that $l \in F^{f}$ and $l\left(x^{i}\right)=l_{i}$ holds.

First, I will show that we have $f\left(x^{i}\right)=l_{i}$. This means that we may apply Theorem 3.9 and Theorem 3.10 to obtain

$$
L_{/}\left(F, x^{i}, x^{k}\right) \leq L /\left(F, x^{j}, x^{k}\right) U,\left(F, x^{i}, x^{j}\right) \quad(i, j, k \in I) .
$$

Moreover, using Theorem 3.12 we obtain

$$
U_{N}\left(F, x^{i}, x^{j}\right) \leq c_{j}\left(x^{i}\right) / c_{j}\left(x^{j}\right) \quad(i, j \in \mathbb{l}) .
$$

Hence

$$
\begin{aligned}
l_{i} & =\max _{k \in I} y_{k} L_{\nu}\left(F, x^{j}, x^{k}\right) \leq \max _{k \in I} y_{k} L /\left(F, x^{j}, x^{k}\right) c_{j}\left(x^{i}\right) / c_{j}\left(x^{j}\right) \\
& =l_{j} c_{j}\left(x^{i}\right) / c_{j}\left(x^{j}\right) \quad(i, j \in I),
\end{aligned}
$$

from which we obtain $f\left(x^{i}\right)=l_{i}$ for all $i \in l$.

Further, we have $f \in F^{f}$, because clearly $f \in F$ and

$$
f\left(x^{i}\right)=l_{i} \geq y_{i} L_{/}\left(F, x^{i}, x^{i}\right)=y_{i} \quad(i \in I) \text {. }
$$

Remark: It is obvious that there is a rationalizing linearly homogeneous frontier production function $f \in F^{f}$ that attains only one lower bound, i.e $f\left(x^{i}\right)=L\left(F^{\prime}, x^{i}\right)$ for a certain $i \in I$. It is not obvious, however, that there exists a function that attains all the bounds $L\left(f, x^{i}\right)$ at once, as is the case for $l$ in Theorem 9.4. As is remarked in Section 8.3 , there exists such a function, because $\mathcal{F} f$ is closed with respect to the pointwise minimum operation.

1 applied Theorem 9.4 to the Dutch industry data and obtained nice results. Before application of the theorem I determined the economical efficiency upper bound for the data without produced output, using the efficiency transformation $\lambda_{x}$ given in Example 3.1. The efficiency upper bound for the assumption of linearly homogeneous producer demand was $e=99.9 \%$, which is much higher than the value of $89.6 \%$, which I obtained when output was included. There is a simple explanation for this. When output is included, one has more information available. This implies that one has in general $\mathcal{F}_{d}(D) \subset \mathcal{F}_{d}(D)$. Hence, the efficiency upper bound for any hypothesis is lower when outputis is observed.

Now, Theorem 9.4 was applied to the transformed data $A_{e} D$ for the obtained economical efficiency bound $e=99.9 \%$. The computed minimal linearly homogeneous frontier production function values $f\left(x^{i}\right)$ are given in 
Pigure 9.1. Below these values the actual produced output $y_{i}$ is shown.

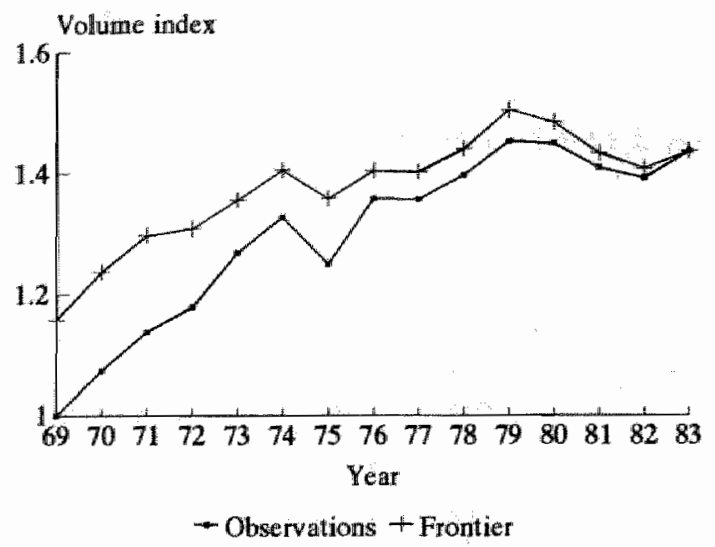

Figure 9.1 Minimal Frontier Production Function

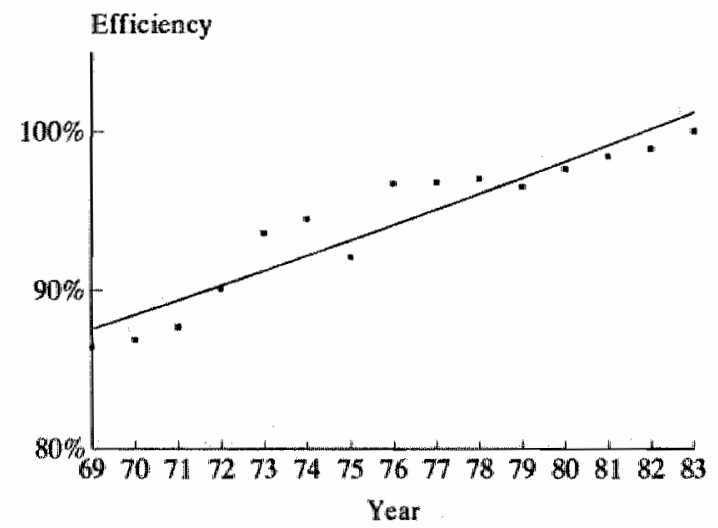

Figure 9.2 Technical Efficiency Upper Bound

From Figure 9.1 we can derive the technical efficiency upper bounds

$$
t_{i}=y_{i} / L\left(F^{f}, x^{i}\right) \quad(i \in I)
$$

as the ratio of the production output $y_{i}$ and the mimimal frontier production output $L\left(F^{\prime}, x^{i}\right)$. These bounds are given in Figure 9.2. If they are not random, but steadily increasing, this may be an indication that there is technical progress. Thus the above theorem is an excellent theorem to test 
whether there is technical progress; using the assumption of linearly homogeneous producer demand. Figure 9.2 contains a logarithmic regression line fit for the values of $t_{i,}$ which is clearly increasing.

Note that there is always at least one observation period for which the output $y_{i}$ equals the minimal frontier function, ie $y_{i}=L\left(F^{f}, x^{i}\right)$. At this point one has $t_{i}=1$ and the minimal frontier production function touches the restrictions that follow from the observations. In case of increasing technical efficiency, this means that one has $t_{i}=1$ for the last observation, as is the case in Figure 9.2,

By Theorem 9.4 , the technical efficiency upper bounds $t_{i}=y_{i} / L\left(F^{f}, x^{i}\right)$ may be interpreted as Hicks neutral change. From the theorem we obtain

$$
\left(c_{i}, x^{i}, y_{i}\right) \in \mathcal{D}_{d}^{o}\left(b_{i} f\right) \quad(i \in I)
$$

for the function

$$
f(x)=\min _{i \in I} L\left(F^{f}, x^{i}\right) c_{i}(x) / c_{i}\left(x^{i}\right) \quad(x \in X)
$$

If we assume that in each observation period $z=0, \ldots, n$, there is a constant fraction $c$ of Hicks nentral technical progress $c$, then we have

$$
t_{i}=t_{0}(1+c)^{i} \quad(i=0, \ldots, n) .
$$

This fraction $c$ may be determined with logarithmic regression, using the relation

$$
\ln t_{i}=\ln t_{0}+i \ln (1+c)=\alpha+i \beta \quad(i=0, \ldots, n),
$$

for the obserwed technical efficiency upper bounds $t_{i}$. The estimation result for the Dutch industry data was $c=1.0 \%\left(R^{2}=0.89\right)$, an increase in technical efficiency of approximately one percent per year.

The subject of Hicks neutral progress in the contexti of linearly homogeneous producer demand will be elaborated later on in this chapter. First I will consider the properties of technical progress.

\subsection{Technícal Progress}

Concerning the assumption of linear homogeneity, the most general assumption of technical progress is a new linearly homogeneous production function for each period, with an increase of output. A necessary and sulficient condition for this simple type of technical progress is given in the following theorem.

Theorem 9.5: Suppose $D=\left\{\left(c_{i}, x^{i}, \gamma_{i}\right)\right\}_{i \in l}$ is a data set, where $c_{i}: X \rightarrow R_{*}$ are linearly homogeneous, $c_{i}\left(x^{i}\right)>0$ and $y_{i}>0$, for all $i \in I$, and $X$ is a come. Then the following conditions are equivalent:

(i) There exists a series of linearly homogeneous production functions $f_{i}$ $i \in I$, such that $f_{j} \leq f_{i}$ if $j \leq i$, and $\left(c_{i}, x^{i}, y_{i}\right) \in \mathcal{D}_{d}^{o}\left(f_{i}\right)$ for all $i \in I$.

(ii) One has

$$
y_{j} \leq y_{i} c_{i}\left(x^{j}\right) / c_{i}\left(x^{i}\right) \quad(i, j \in I: j \leq i) .
$$

Proof (i) $\Rightarrow$ (ii): From (i) and Theorem 9.2, we obtain

$$
\begin{aligned}
& y_{j}=f_{j}\left(x^{j}\right) \leq f_{i}\left(x^{j}\right) \leq y_{i} c_{i}\left(x^{j}\right) / c_{i}\left(x^{i}\right) \quad(i, j \in I: j \leq i) . \\
& \text { (ii) } \Rightarrow \text { (i): Put } \\
& f_{j}(x)=\operatorname{limf}_{i \geq j} y_{i} c_{i}(x) / c_{i}\left(x^{i}\right) \quad(j \in I, x \in X) .
\end{aligned}
$$

For these functions one can easily verify that one has $f_{j} \leq f_{i}$ if $j \leq i_{\text {, }}$ and the condition in (iii) implies that we have $f_{j}\left(x^{j}\right)=y_{j}$ for all $j \in I$. Further, we have clearly $\left(c_{i}, x^{i}, y_{i}\right) \in \mathcal{D}_{d}^{0}\left(f_{i}\right)$ for all $i \in I$. 
Condition (ii) of Theorem 9.5 is used to test the hypothesis of technically progerssing linearly homogeneous producer demand for the Dutch industry data. The result was that one has to remove two periods from the data set in order to get consistency with this hypothesis. Furthernore, we can use Theorem 9.5 to compute an economical efriciency upper bound for the hypothesis technically progressing linearly homogeneous producer demand. The result is $e=97.5 \%$, where the efficiency transformation $A_{e}$ of Example 3.1 is used.

Now, suppose we have data $D=\left\{\left(c_{i}, x\right)\right\}_{i e r}$ avalable which do not include the observed outputs $y_{*}$. Then may ask whether the hypothesis of technically progressing linearly homogeneous producer demand can be falsified. To answer this question we may search for output values $y_{t}$ that obey condition (ii) in Theorem 9.5 above. If such values do exist then there is consistency with the hypothesis of technically progressing linearly homogeneous demand. Using this jdea the following theorem can be derived. It shows that we cannot falsify the bypothesis of technically progressing linearly homogeneous demand when the data do not contain observed outputs.

Theorem 9.6: Suppose $D=\left\{\left(c_{i}, x^{i}\right)\right\}_{\text {ieI }}$ is a countable data set, where $I=\{1,2, \ldots\}, c_{i}: X \rightarrow \mathbb{R}_{*}$ are linearly homogeneows and $c_{i}\left(x^{j}\right)>0$ if $j \leq i$, for all $i, j \in I$, and $X$ is a come. Then there exists a series of linearly homogeneous production functions $f_{i}$, $i \in I_{1}$, such that $f_{j} \leq f_{i}$ if $j \leq i$, and $\left(c_{i}, x^{i}\right) \in \mathcal{D}_{d}\left(f_{i}\right)$ for all $i \in \mathbb{l}$ "

Proof: By Theorem 9.5 there exist technically progressing linearly homogeneous production functions with outputs $y_{i}$ for the data, if condition. (ii) in this theorem is satisfied. To construct such numbers $y_{i}$, let $y_{1}>0$ be an arbitrary number, and put iteratively

$$
y_{i}=\max \left\{y_{j} c_{i}\left(x^{i}\right) / c_{i}\left(x^{j}\right) \mid i, j \in I: j<i\right\} \quad(i=2, \ldots) \text {. }
$$

These numbers satisfy obviously condition (ii) in Theorem 9.5 , which is

$$
y_{j} \leq y_{i} c_{i}\left(x^{j}\right) / c_{i}\left(x^{i}\right) \quad(i, j \in I: j \leq i) \text {. }
$$

Thus by the above proof we can always find output values for the data, such that there is consistency with the hypothesis of technically progressing linearly homogeneous demand. So, it is impossible to falsify the hypothesis. Hence, the specification of linearly homogeneous cost minimization with technical progress does not impose any restriction on the data.

As we have seen above, it may happen in the nonparametric approach that a hypothesis is always satisfied by the data. For example, this was the case in Theorem 8.4. Furthermore, Varian (1988) derived such a result for consumer behaviour. He shows that the axiom of revealed preference places essentially no restriction on behaviour of a subset of goods.

\subsection{Technical Progress for Frontier Production Functions}

When the output data are observed, but we use the notion of a frontier production function, then the approach in the proof of Theorem 9.6 yiclas values of the technical efficiency upper bounds. This is pointed out in the following theorem.

Theorem 9.7: Suppose $D=\left\{\left(c_{i}, x^{i}, y_{i}\right)\right\}_{\text {iet }}$ is a data set, where $I=\{1,2, \ldots\}$, $c_{i}: X \rightarrow \mathbb{R}_{+}$are linearly homogeneous, $c_{i}\left(x^{j}\right)>0$ if $j \leq i$, and $y_{i}>0$, for all $i \in I$, and $X$ is a cone. Let each family $F_{i}$ contain all technically progressing linearly howogeneous frontier production functions for producer demand at period $i \in I$. Thus if thege is a series functions $f_{i}$ ifeI, such that $f_{j} \leq f_{i}$ if $j \leq i$, and $\left(c_{i,} x^{i}, y_{i}\right) \in \mathcal{D}_{d}\left(f_{i}\right)$ for all $i \in I$, then $f_{i} \in \mathcal{F}_{i}$ for every $i \in I$. Now, put $u_{1}=y_{1}$ and iteratively 


$$
w_{i}=\max \left\{u_{j} c_{i}\left(x^{i}\right) / c_{i}\left(x^{j}\right) \mid j \in I: j<i\right\} \cup\left\{y_{i}\right\} \quad(i=2, \ldots)
$$

Then $L\left(F_{i}^{f}, x^{i}\right)=u_{i}$ for all $i \in I$.

Proof: The definition of frontier producer demand $D^{f}$ and Theorem $9, \mathrm{~s}_{3}$ inply together the following. In order to find $u_{i}=L\left(F_{i}^{f}, x^{i}\right)$, we have to search for minimal numbers $u_{i}$ such that

$$
\begin{aligned}
& u_{i} \geq y_{i} \quad(i \in I), \\
& u_{i} \geq u_{j} c_{i}\left(x^{i}\right) / c_{i}\left(x^{j}\right) \quad(i, j \in I: j \leq i y .
\end{aligned}
$$

The numbers $u_{i}$ given in this theorem, are the solution to this problem.

So, the technical upper bounds for linearly homogeneous frontier producer demand with technical progress are $t_{i}=u_{i} / y_{i}, i \in I$, in which the values $u_{i}$ are given in the above theorem. From this theorem it follows that the first technical efficiency upper bound $t_{1}$ is always equal to one.

1 applied Theorem 9.7 to obtain the technical upper bounds for the Dutch industry data. The result contained only two inefficient years with $t_{i}<1$ for the hypothesis of technically progressing linearly homogeneous producer demand: 1975 and 1979 with technical efficiency upper bounds of $97.6 \%$ and $99.5 \%$ respectively.

\subsection{Hicks Neutrall Change}

A test of the hypothesis of Hicks neutral change can be considered as a case where output may be chosen freely. This is in essence the content of the collowing theorem.

Theorem 9.8: Suppose $D=\left\{\left(c_{i}, x^{i}, y_{i}\right)\right\}_{i \in I}$ is a data set, where $c_{i}: X \rightarrow \mathbb{R}_{+}$are linearly homogeneous, $c_{i}\left(x^{i}\right)>0$ and $y_{i}>0$, for all $i \in I$, and $X$ is a cone. Then the following conditions are equivalent:

(i) There exist a linearly homogeneous function $f: X \rightarrow \mathbb{R}$ and numbers $t_{i}>0$, such that $\left(c_{i}, x^{i}, y_{i}\right) \in \mathcal{D}_{d}^{o}\left(t_{i} f\right)$ for all $i \in I$.

(ii) There exists a linearly homogeneous function in $\mathcal{F}_{d}(D)$, where the observed outputs are not used.

Proof (i) $\Rightarrow$ (ii): Suppose the function $f$ and the numbers $t_{i}$ satisfy condition (i). By Theorem 8.6 one has

$$
\left(c_{i}, x^{i}, y_{i}\right) \in \mathcal{D}_{d}^{o}\left(t_{i} f\right) \Leftrightarrow\left(c_{i}, x^{i}, t_{i}^{-1} y_{i}\right) \in \mathcal{D}_{d}^{o}(f) \quad(i \in I)
$$

and we have obviously

$$
\left(c_{i}, x^{i}, t_{i}^{-1} y_{i}\right) \in \mathcal{D}_{d}^{(i)}(f) \Rightarrow\left(c_{i}, x^{i}\right) \in \mathcal{D}_{d}(f) \quad(i \in I)
$$

50

$$
\left(c_{i}, x^{i}, y_{i}\right) \in \mathcal{D}_{d}^{o}\left(t_{i} f\right) \Rightarrow\left(c_{i}, x^{i}\right) \in \mathcal{D}_{d}(f) \quad(i \in I)
$$

and we have thus $f \in \mathcal{F}_{d}(D)$.

(ii) $\Rightarrow$ (i): Suppose $f \in \mathcal{F}_{d}(D)$ is linearly homogeneous. Then Theorem 3.4 and $c_{i}\left(x^{i}\right)>0$ implies $f\left(x^{i}\right)>0$. Now, put $t_{i}=y_{i} / f\left(x^{i}\right)$ for all $i \in I$. Then one has in generall

$$
\left(c_{i}, x^{i}\right) \in \mathcal{D}_{d}(f) \Leftrightarrow\left(c_{i}, x^{i}, f\left(x^{i}\right)\right) \in \dot{\mathcal{D}}_{d^{d}}^{i}(f) \quad(i \in I)
$$

Furthermore, since $f$ is linearly homogeneous, the latter is equivalent to

$$
\left(c_{i}, x^{i}, t_{i} f\left(x^{i}\right)\right) \in \mathcal{D}_{d}^{o}\left(t_{i} f\right) \quad(i \in I)
$$

Finally, we have by assumption

$$
t_{i} f\left(x^{i}\right)=\left(y_{i} / f\left(x^{i}\right)\right) f\left(x^{i}\right)=y_{i} \quad(i \in I) .
$$

We can obtain the nomparametric restrictions on the scale of Hicks neutral 
change from the following theorem.

Theorem 9.9. Suppose $D=\left\{\left(c_{i}, x^{i}, y_{i}\right)\right\}_{\text {ieI }}$ be a finte data set, where $c_{i}, X \rightarrow R_{+}$ are linearly homogeneous, $c_{i}\left(x^{4}\right)>0$ and $y_{3}>0$, for all $i \in I$, and $X$ is a cone. Let $t_{p}>0$ for oll $\in$ I. Then the following conditions are equivalent:

(i) There exists a linearly honogeneous function $f: X \rightarrow \mathbb{R}$ such that

$$
\left(c_{i}, x^{i}, y_{i}\right) \in \mathcal{D}_{d}^{o}\left(t_{i} f\right) \quad(i \in I) .
$$

(it) One has $c_{i}\left(x^{j}\right)>0$ for all $i, j \in I$, and the salues $\phi_{i}=\ln t_{i}$ satisfy

$$
\phi_{i}-\phi_{j} \leq a_{i j}, a_{i j}=\operatorname{In}\left(y_{j} c_{i}\left(x^{i}\right) /\left[y_{i} c_{i}\left(x^{j}\right)\right]\right) \quad(i, j \in I) .
$$

Proof: One thas by Theorem 8.6

$$
\left(c_{i}, x^{i}, y_{i}\right) \in \mathcal{D}_{d}^{o}\left(t_{i} f\right) \Leftrightarrow\left(c_{i}, x^{i}, t_{i}^{-1} y_{i}\right) \in \mathcal{D}_{d}^{o}(f) \quad(i \in I) .
$$

So, by Theorem 9.1 , we have (i) equivallent to

$$
t_{j}^{-1} y_{j} \leq t_{i}^{-1} y_{i} c_{i}\left(x^{j}\right) / c_{i}\left(x^{i}\right) \quad(i, j \in I) .
$$

This is equivalent to

$$
t_{i} / t_{j} \leq y_{i} c_{i}\left(x^{j}\right) /\left[y_{j} c_{i}\left(x^{i}\right)\right] \quad\left(i_{i} j \in I\right),
$$

from which we obtain the inequalities in (ii), by taking the logarithm at both sides. Furthermore, because all $t_{i}$ and $y_{i}$ are positive, we obtain from (i) also $c_{i}\left(x^{j}\right)>0$ for all $i, j \in I$.

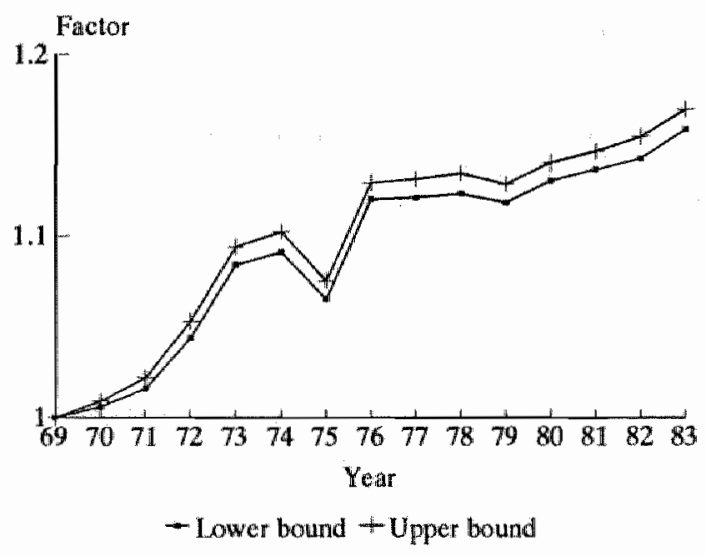

Figure 9.3 Bounds Hicks Neutral Change

Figure 9.3 displays lower and upper bounds for the scale of Hicks neutral change. The bounds follow from condition (ii) in Theorem 9.9 and they are obtained from the transformed Dutch industry data $A_{\varepsilon} D$, where $e=99.9 \%$. The latter is the efficiency upper bound $e$ for the hypothesis of linearly homogeneous Hicks neutral changing producer demand. As is stated in Theorem 9.8, this efficiency upper bound can be obtained by testing the hypothesis of linearly homogeneous producer demand without using the observed output. 


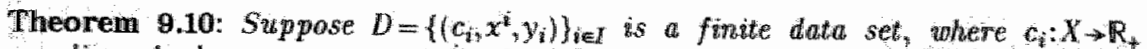
are lomearly homogeneous, $c_{i}\left(x^{i}\right)>0$ and $y_{i}>0$, for all $i \in I$, and $X$ is a cone. Let $t_{i}>0$ for all $i \in I$. Then the following conditurons are equivolent;

(1) One has $t_{i} \leq t_{j}$ if $i \leq j$, and there exists a linearly homogeneous function $f: X \rightarrow \mathbb{R}$ such that $\left(c_{i}, x_{i}^{i} y_{i}\right) \in \mathcal{D}_{d}^{a}\left(t_{i}\right)$ for all $i \in l$.

(ii) One has $c_{i}\left(x^{j}\right)>0$ for all $i_{j} j \in I$, and the values $\phi_{i}=\ln t_{i}$ satisfy

$$
\phi_{i}-\phi_{j} \leq \min \left\{a_{i j}, b_{i j}\right\} \quad(i, j \in I)
$$

where

$$
\begin{aligned}
& a_{i j}=\ln \left(y_{j} c_{i}\left(x^{i}\right) /\left[y_{i} c_{i}\left(x^{j}\right)\right]\right) \quad(i, j \in I), \\
& b_{i j}= \begin{cases}0 & (i, j \in I: i \leq j), \\
\infty & \text { (otherwise). }\end{cases}
\end{aligned}
$$

Proof: This is similar to the proof of Theorem 9.9 with the additional condition that one has $t_{i} \leq t_{j}$ if $i \leq j$, which is equivalent to

$$
t_{i} / t_{j} \leq 1 \quad(i, j \in I: i \leq j)
$$

From this we obtain the additional inequalities for $b_{i j}$ in (ii), by taking the logarithm at both sides.

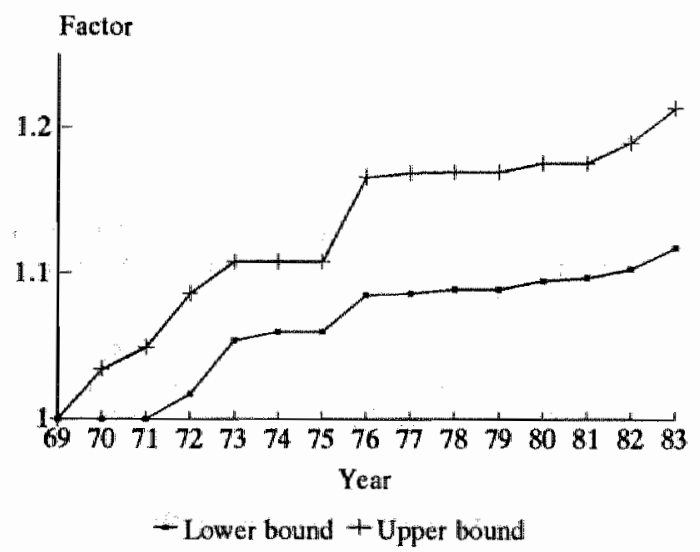

Figure 9.4 Bounds Hicks Neutral Progress

We can use condition (ii) of the above theorem to test the hypothesis of Hicks neutral progress. Concerning the Dutch industry datia the result was as follows. Four observation years have to be removed to obtain consistency with the hypothesis of Hicks neutral progressing linearly homogeneous demand. The economical efficiency upper bound for this hypothesis was $e=97.5 \%$, where again the efficiency transformation $A_{e}$ from Example 3.1 is used.

Using condition (ii) in Theorem 9.10, we may obtain lower and upper bounds for the scale of Hicks neutral progress. Figure 9.4 displays these lower and upper bounds for the transformed Dutch industry data $A_{e} D$, where $e$ is equal to the efficiency upper bound $e=97.5 \%$. 


\section{Q.8 Nentral Change}

The assumption of neutral change has in case of lnearly homogeneous producer denand a relatively simple solution when output is observed. The solution becomes more difficul when output is not observed. Then both the scale of progress and the output may be chosen freely, which doubles the number of free variables. In the following theorem it is assumed that output is observed.

Theorem 0.11: Suppose $D=\left\{\left(c_{i}, x^{i}, y_{i}\right)\right\}_{\text {ef }}$ is a data set, where $c_{i}: X_{1} \times X_{2} \rightarrow \mathbb{R}_{4}$ are lincarly homogeneous, $c_{i}\left(x^{i}\right)>0$ and $y_{i}>0$, for all $i \in I$, and $X_{1}$ and $X_{2}$ are cones Let $t_{i}>0$ for $u$ l $E \mathbb{L}$. Then the following conditions are equivalent:

(i) There exists a linearly homogeneous function $f: X_{1} \times X_{2} \rightarrow \mathbb{R}$ such that all functions

$$
f_{i}(x)=f\left(x_{1}, t_{i} x_{2}\right) \quad\left(i \in I, x \in X_{1} \times X_{2}\right)
$$

satisfy $\left(c_{i}, x^{i}, y_{i}\right) \in \mathcal{D}_{(d)}^{o}\left(f_{i}\right)$.

(ii) One has

$$
y_{j} \leq y_{i} c_{i}\left(x_{1}^{j}, t_{i}^{-1} t_{j} x_{2}^{j}\right) / c_{i}\left(x^{i}\right) \quad(i, j \in I) .
$$

Proof: By Theorem 8.10 we have (i) equivalent to

$$
\left(c_{i}^{t},\left[x_{1}^{i}, t_{i} x_{2}^{i}\right], y_{i}\right) \in \mathcal{D}_{d}^{o}(f) \quad(i \in I)
$$

where

$$
c_{i}^{t}\left(x_{1}, x_{2}\right)=c_{i}\left(x_{1}, t_{i}^{-1} x_{2}\right) \quad\left(i \in I, x_{1} \in X_{1}, x_{2} \in X_{2}\right) .
$$

By Theorem 9.1 this is equivalent to

$$
y_{j} \leq y_{i} c_{i}^{t}\left(x_{1}^{j}, t_{j} x_{2}^{j}\right) / c_{i}^{b}\left(x_{1}^{i}, t_{i} x_{2}^{i}\right) \quad(i, j \in I) .
$$

This, finally, is equivalent to (ii).

If we consider price and quantity data then there is a simple test for newtral change. This test is given in the following theorem, which follows directly from the above theorem.

Theorem 9.12: Suppose $D=\left\{\left(c_{i}, x^{i}, y_{i}\right)\right\}_{\text {ie }}$ is a data set, where $c_{i}: X_{1} \times X_{2} \rightarrow \mathbb{R}_{+}$are linearly homogeneous, $c_{i}\left(x^{i}\right)>0$ and $y_{i}>0$, for all $i \in I$, and $X_{1}$ and $X_{2}$ are cones. Suppose all functions $c_{i}$ are of the form

$$
c_{i}(x)=v_{i}\left(x_{1}\right)+w_{i}\left(x_{2}\right) \quad\left(i \in I, x \in X_{1} \times X_{2}\right)
$$

where $v_{i}: x_{1} \rightarrow \mathbb{R}_{+}$and $w_{i}: X_{2} \rightarrow \mathbb{R}_{+}$are linearly homogeneous. Let $t_{i}>0$ for all $i \in I$. Then the following conditions are equivalent:

(i) There exists a linearly homogeneous function $f: X_{1} \times X_{2} \rightarrow R_{4}$ such that all functions

$$
f_{i}(x)=f\left(x_{1}, t_{i} x_{2}\right) \quad\left(i \in I, x \in X_{1} \times X_{2}\right)
$$

sidisfy $\left(c_{i}, x^{i}, y_{i}\right) \in \mathcal{D}_{d}^{i}\left(f_{i}\right)$.

(iii) The values $\phi_{i}=\ln \psi_{i}$ satisfy

$$
\phi_{i}-\phi_{j} \leq a_{i j} \quad\left(i_{i} j \in I\right)
$$

where

$$
a_{i j}= \begin{cases}\left.\ln \left(w_{i}\left(x_{2}^{j}\right) / \| c_{i}\left(x^{i}\right) y_{j} / y_{i}-v_{i}\left(x_{\|}^{j}\right)\right]\right) & \left(i, j \in I: c_{i}\left(x^{i}\right) y_{j} / y_{i}>v_{i}\left(x_{1}^{j}\right)\right), \\ \infty & \text { (otherwise). }\end{cases}
$$

Proof: Condition (i) is by Theorem 9.11 equivalent to

$$
y_{j} \leq y_{i} c_{i}\left(x_{1}^{j}, t_{i}^{-1} t_{j} x_{2}^{j}\right) / c_{i}\left(x^{i}\right) \quad(i, j \in I) \text {. }
$$


Hence, condition (i) is equivalent to

$$
y_{j} \leq y_{i}\left[v_{i}\left(x_{1}^{j}\right)+t_{i}^{-1} t_{j} w_{i}\left(x_{2}^{j}\right)\right] / c_{i}\left(x^{i}\right) \quad(i, j \in I),
$$

and these inequalities are in turn equivalent to

$$
\ln t_{i}-\ln t_{j} \leq a_{i j} \quad(i, j \in I) \text {. }
$$

Condition (ii) of Theorem 9.12 is used to test neutral change of labour for" the Dutch industry data. The result was that four observations have to be removed in order to get consistency with the hypothesis of neutral changing linearly homogeneous producer demand.

We may also use Theorem 9.12 to derive an economical efficiency upper bound for the hypothesis of neutral changing linearly homogeneous producer demand. There is, however, a small problem with the choice of an efficiency transformation. In Theorem 9.12 it is assumed that we may write the cost functions in the form

$$
c_{i}(x)=v_{i}\left(x_{1}\right)+w_{i}\left(x_{2}\right) \quad\left(x \in X_{1} \times X_{2}\right)
$$

where $w_{i}$ and $w_{i}$ are linearly homogeneous functions. Thus to apply this theorem, we have to choose a suitable efficiency transformation, such that the resulting cost functions satisfy this condition. An example is the following efficiency transformation.

Example 9.1 Suppose $\left(c, x^{*}\right)$ is a data observation such that

$$
c(x)=v\left(x_{1}\right)+w\left(x_{2}\right) \quad\left(x \in X_{1} \times X_{2}\right)
$$

where $v$ and $v$ are linearly homogeneous functions. Define, using the efficiency transformation $A_{e}$ given in Example 3.1, the functions $v^{e}$ and $w^{e}$ as follows

$$
\left(v^{e}, x_{1}^{i}\right)=\Lambda_{e}\left(v, x_{1}^{i}\right), \quad\left(w^{e}, x_{2}^{i}\right)=\Lambda_{e}\left(w, x_{2}^{i}\right) .
$$

Then we may define the efficiency transformation $\hat{A}_{e}$ as follows

$$
\hat{\lambda}_{e}\left(v+w_{,} x^{n}\right)=\left(v^{e}+w^{e}, x^{\prime}\right)
$$

Such an efficiency transformation transforms linearly homogeneous functions $v$ and $w$ into linearly homogeneous functions $v^{e}$ and $w^{e}$ respectively.

The above efficiency transformation $\hat{A}_{e}$ resulted for neutral change of labour in an efficiency upper bound of $e=99.8 \%$, and for neutral change of capital in $e=99.9 \%$.

Using the transformed data $\hat{A}_{e} D$ for the computed economical efficiency $\epsilon_{*} \mathbb{1}$ computed nomparametric restrictions on the scale of neutral change for labour. It is assumed that this scale is equal to one in the first observation year. The restrictions follow from the weak closure $A^{*}$ of $A=\left[a_{i j}\right]$, with $A$ defined as in Theorem 9.12. As is shown in appendix C.5, the inequalities

$$
\phi_{i}-\phi_{j} \leq a_{i j} \quad(i, j \in I)
$$

in Theorem 9.12 imply the inequalities

$$
-a_{j i}^{*} \leq \phi_{i}-\phi_{j} \leq a_{j i}^{*} \quad(i, j \in I) \text {. }
$$

Since we had $\phi_{i}=\ln t_{i}$, where $t_{i}$ is the scale of neutral change, this yields the following restrictions on the scale of neutral change

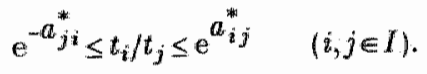

Figure 9.5 displays these bounds for $j=1$, the index of the first observation. The figure shows that the resulting bounds for neutral changing labour are very narrow. 


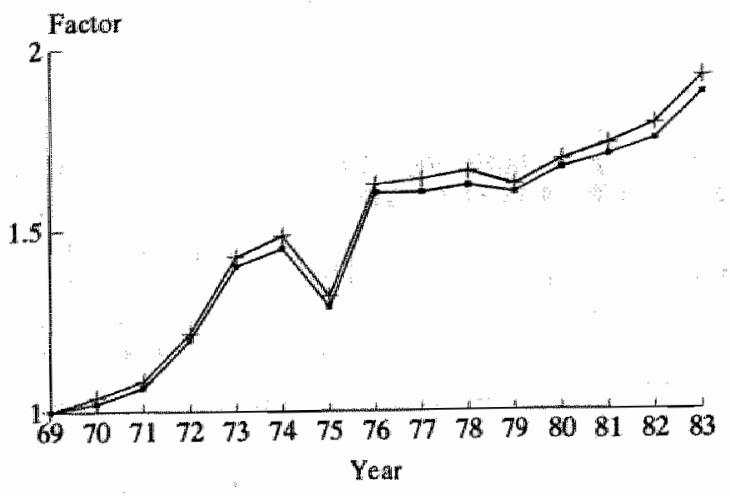

- Lower bound + Upper bound

Figure 9.5 Bounds Neutral Change Labour

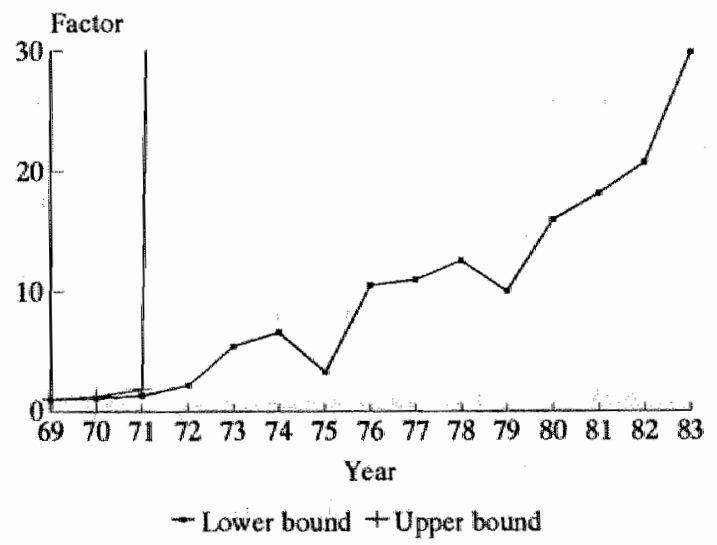

Figure 9.6 Bounds Neutral Change Capital

Concerning neutral change of capital there was only one outlying observation, which has to be removed in orcler to get consistency with the hypothesis of neutral changing linearly homogeneous producer demand. The economical efficiency upper bound was $e=99.9 \%$.

I computed - in the same way as for labour - the nonparametric bounds for the scale of neutral change of capital. In this case the results were less informative. As shown in Figure 9.6, the upper bound increased very fast to values which exceed any informative restriction. What is the interpretation 
of the occurrence of an infinite upper bound? An infinite upper bound means that we have

$$
t_{i} / t_{j}<\mathrm{e}^{a_{i j}^{*}}=\infty
$$

which is equivalent to $a_{i j}=\infty$. In the following I will consider properties that may be a cause for the occurrence of such infinite upper bounds in case $y=1$, the index of the first obserwation.

Suppose the data are consistent with the hypothesis of technically progressing linearly homogeneous producer demand. Ther we have by "Theorem 9.5 the relation

$$
y_{j} \leq y_{i} c_{i}\left(x^{j}\right) / c_{i}\left(x^{i}\right) \quad(i, j \in I: j \leq i) .
$$

This means that the inequalities, given in Theorem 9.5, imply that we have

$$
c_{i}\left(x^{i}\right) y_{j} / y_{i}-c_{i}\left(x^{j}\right) \leq 0 \quad(i, j \in I: j \leq i) \text {. }
$$

This makes it likely that we have

$$
c_{i}\left(x^{i}\right) y_{j} / y_{i}-c_{i}\left(x^{j}\right)+w_{i}\left(x_{2}^{j}\right)=c_{i}\left(x^{i}\right) y_{j} / y_{i}-v_{i}\left(x_{1}^{j}\right) \leq 0 \quad(i, j \in \mathbb{I}: j \leq i),
$$

especially when the added cost $w_{i}\left(x_{2}\right)$ is relatively small. The latter applies to capital and not to labour, because capital and labour costs are approximately $3 \%$ and $25 \%$ of the total costs $c_{i}\left(x^{i}\right)$ respectively. Now, because we have the equivalence

$$
c_{i}\left(x^{i}\right) y_{j} / y_{i}-v_{i}\left(x_{1}^{j}\right) \leq 0 \Leftrightarrow a_{i j}=\infty \quad(i, j \in I)
$$

it is likely that we obtain $a_{i j}=\infty$ for $j<i$. In that case every path from $j$ to $i$ with $j<i$ always encounters an infinite value $a_{k l}=\infty$, with $k<l$. So, we have then $a_{i j}=\infty$ for $j<i$. This in turn means that we find infinite upper bounds

$$
t_{i} / t_{1}<\mathrm{e}^{a_{i 1}^{*}}=\infty \quad(i>1) .
$$

Summarizing the above. When the data are approximately consigtent with technically progressing linearly homogeneous producer demand and when the neutral changing costs $w_{i}\left(x_{2}^{j}\right)$ are relatively small, then we may expect the occurrence of infinite upper bounds for $t_{i} / t_{1}$.

We observed this phenomenon for neutral changing capital in Figure 9.6. It does not appear in Figure 9.5, for meutral changing labour, because labour costs are substantially higher than capital costs.

We may apply the theory derived for neutrall change, to the case of neutral progress. Then we have only to add the assumption that the scale is increasing. "This leads to the following theorem.

Theorem 9.13: Concerning Theorem 9.12, the following condations are equivalent:

(i) Theorem 9.12 (i) is satisfied and one has $t_{j} \leq t_{i}$ if $j \leq i$.

(ii) The values $\phi_{i}=\ln t_{i}$ satisfy

$$
\phi_{i}-\phi_{j} \leq \min \left\{a_{i j}, b_{i j}\right\} \quad(i, j \in I)
$$

where $a_{i j}$ is given in Theorem 9.12 (ii) and

$$
b_{i j}= \begin{cases}0 & (i, j \in I: i \leq j), \\ \infty & (\text { otherwise }) .\end{cases}
$$

Proof: This is a version of Theorem 9.12 with the additional assumption that $t_{j} \leq t_{i}$ if $j \leq t$.

I applied condition (ii) of the above theorem to determine the scale of neutral progress of labour and capital for the Dutch industry data. In order 
to accept the hypothesis of neutral progress of labour and capital there are five and three observations respectively, that have to be removed. The economical efficiency upper bounds were both equal to $97.5 \%$.

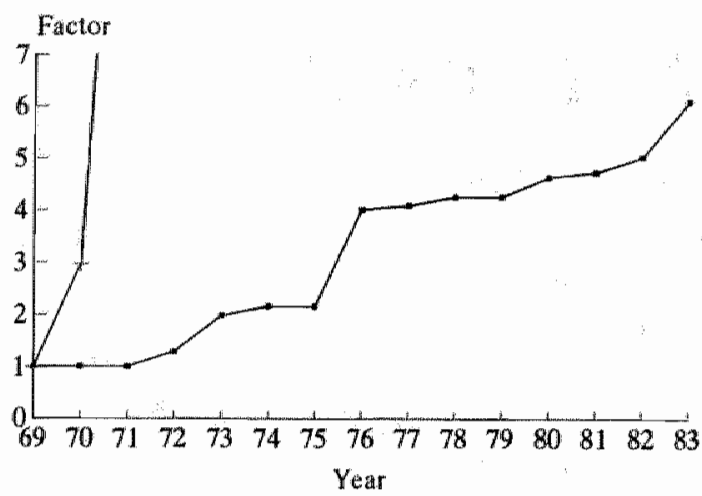

- Lower bound + Upper bound

Figure 9.8 Bounds Neutral Progress Capital

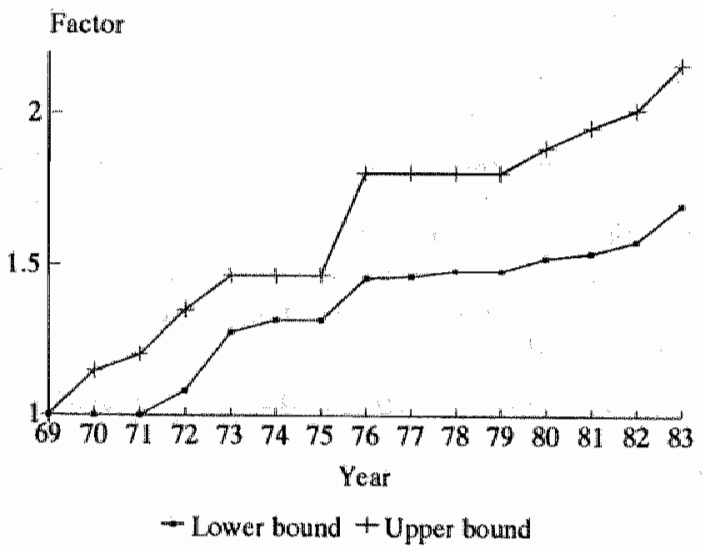

Figure 9.7 Biounds Neutral Progress Labour

The nonparametric restrictions on the scale of neutral progress are computed in the same way as they were computed for neutral change. The results are displayed in Figure 9.7 and 9.8 . It appears that they are similar to the earlier obtained results of neutral change. 


\subsection{Introduction}

In this chapter we consider the existence of homothetic production functions for producer data. This case weakens the assumption of linear homogeneity, treated in the previous chapter, because a homothetic function is a monotonic transformation of a linearly homogeneous function. In case of unobserved output one cannot distinguish between demand behaviour concerning linearly homogeneous production functions or demand behaviour concerning homothetic functions. So, since linearly homogeneous demand behaviour is already treated in Chapter 9, I will assume in the present chapter that output is observed. The two main questions that are answered are:

1. When does a homothetic production function exist for producer demand data?

2. What are the restrictions on the corresponding set of underlying linearly homogeneous functions?

\subsection{Existence of a Homothetic Production Function}

Homotheticity is a generalization of linear homogeneity. The definition that is given by Varian $(1983,1984)$, and used in this book, is the following.

Definition: A function $h$ is said to be homothetic if it can be written as $h=m \circ f$, where $m: \mathbb{R} \rightarrow \mathbb{R}$ is a strictly increasing function and $f$ linearly homogeneous.

Remark: There is another common used definition of homotheticity. Shephard $(1953,1970)$ defines a function $h$ to be homothetic if there exists an increasing function $v: \mathbb{R} \rightarrow \mathbb{R}$ such that $f=\eta$ h is linearly homogeneous. A function of the form $h=m$ of satisfies the homotheticity definition given by Shephard, if $m: \mathbb{R} \rightarrow \mathbb{R}$ is strictly increasing and $f$ is linearly homogeneous. Then the inverse function $m^{-1}$ is a well-defined increasing function. Furthermore, for $v=m^{-1}$ one has $v_{0} h=f$ linearly homogeneous.

A homothetic function $h=$ mof rationalizes producer demand, when the following conditions are satisfied:

1. the data without output is rationalized by the linearly homogeneous function $f$;

2. the linearly homogeneous output values are mapped on the output observations by the monotonic transformation $m$.

Thus in combination with the idea behind Theorem 9.3 leads to the following existence theorem for a rationalizing homothetic production function.

Theorem 10.1: Suppose $D=\left\{\left(c_{i}, x^{i}, y_{i}\right)\right\}_{i \in I}$ is a finite data set of producer behwviour, where $c_{i}: X \rightarrow \mathbb{R}_{+}$are linearly homogeneous and $c_{i}\left(x^{\hat{\beta}}\right)>0$, for all $i \in I$, and $X$ is a cone. Then the following conditions are equivalent:

(i) There exists a homothetic function $h \in \mathcal{F}_{d}^{0}(D)$.

(ii) There exists a homothetic function $h \in \mathcal{F}_{\mathrm{c}}(D)$.

(iii) There exist numbers $\phi_{i}$ such that

$$
\phi_{i}-\phi_{j} \leq \min \left\{a_{i j}, b_{i j}\right\}, \phi_{i}-\phi_{j}<c_{i j} \quad(i, j \in I),
$$

where

$$
a_{i j}=\ln \left(c_{j}\left(x^{i}\right) / c_{j}\left(x^{j}\right)\right) \quad(i, j \in I)
$$




$$
\begin{aligned}
& b_{i j}= \begin{cases}0 & \left(w_{i} j \in I: y_{i} \leq y_{j}\right), \\
\infty & (\text { otherwise }),\end{cases} \\
& \epsilon_{i j}= \begin{cases}0 & \left(i, j \in \mathbb{I}: y_{i}<y_{j}\right), \\
\infty & \text { (otherwise). }\end{cases}
\end{aligned}
$$

Proof $(1) \Rightarrow$ (ii) Trivial.

$(i) \Rightarrow\left(\right.$ init). Suppose $h=m o f \in F_{C}^{a}(D)$ is a homothetic function, where $m$ is strictly increasing and $f$ is linearly homogeneous. Then $f\left(x^{i}\right)>0$ for all $i \in I$ by Theorem 3.4 , and by Theorem 3.11 we have

$$
f\left(x^{i}\right) / f\left(x^{j}\right) \leq c_{j}\left(x^{i}\right) / c_{j}\left(x^{j}\right) \quad(i, j \in 1) \text {. }
$$

Moreover, m strictly increasing means that we have

$$
y_{j}=h\left(x^{j}\right) \geq h\left(x^{i}\right)=y_{i} \Leftrightarrow f\left(x^{j}\right) \geq f\left(x^{i}\right) \quad(i, j \in I) .
$$

So (iii) follows using $\phi_{i}=\ln f\left(x^{i}\right)$.

(iii) $\Rightarrow$ (i): Suppose that the numbers $\phi_{i}=\ln f_{i}$ satisfies (iii). Put

$$
f(x)=\min _{i \in I} f_{i} c_{i}(x) / c_{i}\left(x^{i}\right)
$$

and let $m: \mathbb{R} \rightarrow \mathbb{R}$ be a strictly increasing function such that $m\left(f_{i}\right)=y_{i}$ for all $i \in 1$. Such a function $m$ exists, because (iii) implies that we have

$$
f_{i} \geq f_{j} \Leftrightarrow y_{i} \geq y_{j} \quad(i, j \in I) \text {. }
$$

Now; mof is clearly a homothetic function. Further, (iil) implies that we have

$$
f\left(x^{i}\right)=\min _{j \in I} f_{j} c_{j}\left(x^{i}\right) / c_{j}\left(x^{j}\right)=f_{i} \quad(i \in I)
$$

Hence, $f \in \mathcal{F}_{d}(D)$ and also $m o f \in \mathcal{F}_{d}^{o}(D)$.

A theorem that states also necessary and sufficient conditions for homothetic producer demand is given by Varian (1984). His theorem is inspired by a theorem given in Hanoch and Rothschild (1972). However, both these theorems contain a small error in the given conditions. Varian does not require

$$
\phi_{i}-\phi_{j} \leq b_{i j} \quad(i, j \in I)
$$

and Haroch and Rothschildt do not require the condition

$$
\phi_{i}-\phi_{j}<c_{i j} \quad(i, j \in I) \text {. }
$$

Varian proves that his condition is sufficient using the inverse of an increasing function $m$. However, such an inverse function is only well-defined If $m$ is strictly increasing.

Theorem 10.1 is an improvenent of both earlier given theorems. Besides the correction of this error, cost functions may be used instead of prices.

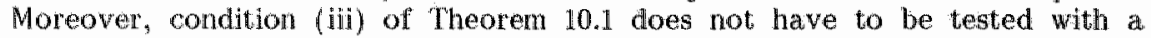
time consuming linear program as in the case of the condition given in Hanoch and Rothschildt (1972), and later in Varian (1984). To test condition (iii) of Theorem 10.1 one may use a simple shortest path test as is shown in Theorem C.3, given in Appendix C.

We may extend Theorem 10.1 to the case where the data set $D$ contains an infinite number of elements. In that case we only can prove the implications (i) $\Rightarrow$ (ii) $\Rightarrow$ (iii). The proof (iii) $\Rightarrow$ (i) is not valid for infinite data sets: it does not result necessarily in a strictly increasing function $m$, but 
wre may derive an increasing function $m$.

We may use condition (iii) of Theorem 10.1 to test the Hyothosich pat homothetic producer demand. This resulted for the Dutch industry date an a economical efficiency upper bound of $e=99.5 \%$, using the whetengy

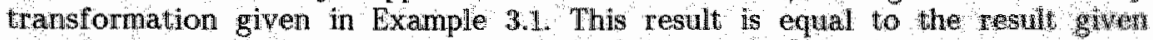
in Chapter 8, where the hypothesis of producer demand without any testriction is considered. To accept the hypothesis of homothetic producer denand, we have to remove four observations, while it only were two observathons in: Chapter 8 . Still it seems that the restriction of homotheticity is not a very strong restriction.

\subsection{Restrictions on the Underlying Linearly Homogeneous Function}

In this section I will construct the relative upper bound for the set of underlying linearly homogeneous functions. The theory is closely related to Chapter 3, which treats restrictions on linearly homogeneous consumer demand: A difficulty in this case is that the relative upper bounds do not necessarily yield a rationalizing function, as was the case for linearly homogeneous consumer demand with unobserved produced output. However, we can use the relative upper bounds for the construction of a rationalizing function, assuming the data set is finite.

Theorem 10.2: Suppose $D=\left\{\left(c_{i}, x^{i}, y_{i}\right)\right\}_{\text {ieI } I}$ is a finite data set of producer behaviour, where $c_{i}: X \rightarrow \mathbb{R}_{+}$are linearly homogeneows and $c_{i}\left(x^{i}\right)>0$, for all $i \in l$, and $X$ is a cone. Put

$$
\begin{aligned}
& F=\{f: X \rightarrow \mathbb{R} \mid f \text { linearly homogeneous, } \\
& \text { there is a monotonic function } \left.m \text { such that mof } \in \mathcal{F}_{d}^{o}(D)\right\} .
\end{aligned}
$$

If $F \neq$ then all functions of the form

$$
g(x)=\sum_{i \in I} \alpha_{i} U /\left(F, x, x^{i}\right), \quad(x \in X)
$$

where $\alpha_{i}>0$ for all $i \in I$, are elenent of $F$.

Proof: Pat

$$
F^{*}=\left\{f \in \mathcal{F}_{d}(D) \mid f \text { linearly homogeneous }\right\},
$$

where the observed outputs are not used. Now, by Theorem 3.12 one has $U_{f}\left(F_{,}, x^{i}\right) \in F^{\prime}$ for all $i \in I$, and by Theorem $5.3(c)$ we have in that case $g \in F^{*}$. Furthermore, the definition of $F$ and $V_{*}$ implies that one has

$$
\begin{aligned}
& U_{\lambda}\left(F, x^{k}, x^{i}\right) \geq U_{\lambda}\left(F, x^{j}, x^{i}\right) \quad\left(j, k \in I: y_{k} \geq y_{j}\right), \\
& U_{\lambda}\left(F, x^{k}, x^{j}\right)>1=U_{N}\left(F, x^{j}, x^{j}\right) \quad\left(j, k \in I: y_{k}>y_{j}\right),
\end{aligned}
$$

which means

$$
\begin{aligned}
y_{k} \geq y_{j} & \Rightarrow \forall i: U_{/}\left(F, x^{k}, x^{i}\right) \geq U_{/}\left(F, x^{j}, x^{i}\right) \Rightarrow g\left(x^{k}\right) \geq g\left(x^{j}\right), \\
y_{k}>y_{j} & \Rightarrow \forall i: U_{/}\left(F, x^{k}, x^{i}\right) \geq U_{/}\left(F, x^{j}, x^{i}\right) \text { and } U_{/}\left(F, x^{k}, x^{j}\right)>U_{\lambda}\left(F_{,}, x^{j}, x^{j}\right) \\
& \Rightarrow g\left(x^{k}\right)>g\left(x^{j}\right)
\end{aligned}
$$

for arbitrary $k, j \in I$. So, we hawe

$$
y_{k} \geq y_{j} \Leftrightarrow g\left(x^{k}\right) \geq g\left(x^{j}\right) \quad(k, j \in I) .
$$

Hence, there exists a monotonic transformation $m$ such that $m \circ g\left(x^{i}\right)=y_{i}$ for all $i \in I$, and, since $g \in F^{\prime}$, one has thus $g \in F_{\text {. }}$

Note that we have not necessarily $U,\left(F, x^{i}\right) \in F$ in the above theorem, because the ordering of both series values $U,\left(F, x^{j}, x^{i}\right)$ and $y_{j}$, for all $j \in I$, may be different. The reason is that the relation 


$$
y_{k}>y_{j} \Rightarrow U_{l}\left(F_{,}, x^{k}, x^{i}\right)>U_{j}\left(F_{,}, x^{j}, x^{i}\right)
$$

is not necessarily valid. We can only prove that we have

$$
y_{k} \geq y_{j} \Rightarrow U_{/}\left(F_{,}^{*} x^{k}, x^{i}\right) \geq U /\left(F_{*} x^{j}, x^{i}\right) \text {. }
$$

Finally I give a theorem, which determines the relative upper bound of the underlying linearly homogeneows functions that rationalize homothetic producer demand. The theorem is closely related to Theorem 3.12 .

Theorem 10.3: Suppose $D=\left\{\left(c_{i}, x^{i}, y\right)\right\}_{i e I}$ is a fanite data set of producer behaviour, where $c_{i}: X \rightarrow \mathbb{R}_{+}$are linearly homogeneous and $c_{i}\left(x^{i}\right)>0$, for all $i \in l$, and $X$ is a cone. Put

$$
\begin{aligned}
& F=\{f: X \rightarrow \mathbb{R} \mid f \text { linearly homogeneous, } \\
& \text { there is a monotonic function } \left.m \text { such that mof } \in \mathcal{F}_{d}^{o}(D)\right\} \text {. } \\
& a_{i j}=\ln \left(c_{j}\left(x^{i}\right) / c_{j}\left(x^{j}\right)\right) \quad(i, j \in I), \\
& b_{i j i}= \begin{cases}0 & \left(i, j \in I: y_{i} \leq y_{j}\right)_{n} \\
\infty & \text { (otherwise }),\end{cases} \\
& d_{i j}=\min \left\{a_{i j}, b_{i j}\right\} \quad\left(i_{i}, j \in I\right) \text {. }
\end{aligned}
$$

If $F \neq \varnothing$ then one has

$$
\begin{aligned}
& U_{N}\left(F_{,}, x, x^{j}\right)=\inf _{i \in I}\left[c_{i}(x) / c_{n}\left(x^{i}\right)\right] \mathrm{e}^{d_{i j}^{*}} \quad(j \in I, x \in X), \\
& U_{/}\left(F_{*}, x^{i}, x^{j}\right)=e^{d_{i j}^{*}}, v_{N}\left(F, x^{i}, x^{j}\right)^{-\pi}=L_{/}\left(F, x^{j}, x^{i}\right) \quad(i, j \in I) .
\end{aligned}
$$

Proof: This is a similar statement as Theorem 3.12 with this difference, the additional output information

$$
U_{N}\left(F, x^{i}, x^{j}\right) \leq 1 \quad\left(i, j \in I: y_{i} \leq y_{j}\right)
$$

is available. An outline of the proof is as follows. Put

$$
f_{j}(x)=\inf _{i \in I}\left[c_{i j}(x) / c_{i}\left(x^{i}\right)\right] \mathrm{e}^{d_{i j}^{*}} \quad(j \in I, x \in X)
$$

and let $k \in I$. Then Theorem 10.2 and $F \neq \emptyset$ implies that one has

$$
f_{k}+\alpha \sum_{j \neq k} f_{j} \in F \quad(\alpha>0) \text {. }
$$

Now, letting $\alpha * 0$, and noting that $f_{k}\left(x^{k}\right)=1$, we obtain

$$
U_{i}\left(F, x, x^{k}\right) \geq f_{k}(x) \quad(x \in X) \text {. }
$$

Futhemore, we have

$$
U_{/}\left(F, x, x^{k}\right) \leq f_{k}(x) \quad(x \in X)
$$

which we can show in a similar way as in the proof of Theorem 3.12 , and thus $v_{1}\left(f, x, x^{k}\right)=f_{k}(x)$. The remaining part of the proof follows in a similar way as in the proof of Theorem 3.12 .

The above theorem is useful to obtain an impression of the monotonic transformation $m$ of a homothetic function mof, where $f$ is linearly homogeneous. When we observe the output values of the function mof, we may estimate the values of $f$ using the above theorem. Figure 1.0 .1 shows results of application of the theorem to the Dutch industry data. Because the Dutch industry data were not compatible with the hypothesis of homotheticity, an afficiency transfomation is applied to the data. The transformation given in Example 3.1 is used with $e=99.5 \%$. The latter is the economical efficiency upper bound that was found in the previous section. Figure 10.1 displays the lower and upper bounds 


$$
\mathrm{e}^{-d_{1 i}^{*}} \leq f\left(x^{i}\right) / f\left(x^{\mathrm{d}}\right) \leq \mathrm{e}^{d_{i 1}^{*}} \quad(i, j \in I)
$$

with the first year as base year. In this figure we use indices such that $y_{1}=1$ and $f\left(x^{1}\right)=1$. For every observed output $y_{i}=$ mof $\left(x^{6}\right)$, on the vertical axis, the figure shows two points, representing the corresponding left and right bound of $f\left(x^{4}\right)$, on the horizontal axis. From this figure it appears that the left part of the monotonic function is not concave. This will have implications concerning the profit maximization hypothesis, considered in Part III. Concerning price and quantity data we can only observe the concave part of a profit maximizing production function. Later on we shall discover that the hypothesis of homothetic profit maximization is indeed seriously violated.

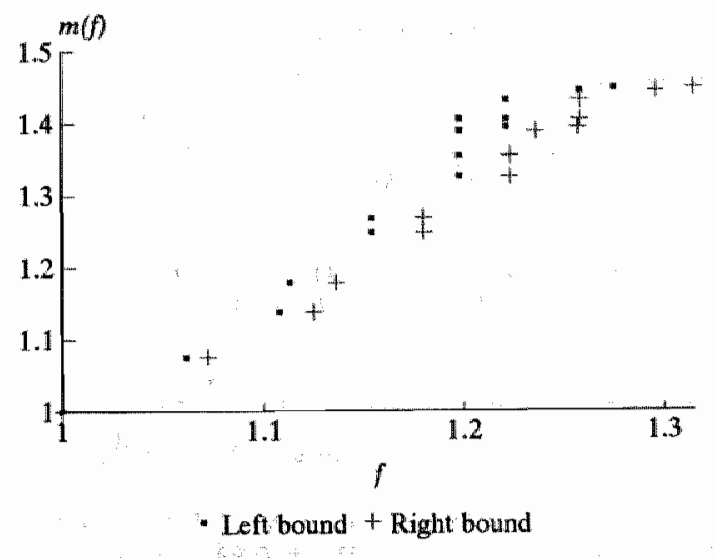

Figure 10.1 Bounds Monotonic Function 


\section{WEAKLY SEPARABLE PRODUCER DEMAND}

\subsection{Introduction}

Conditions for the existence of a weakly separable production function in case of observed output can be derived easily from the results for unotserved output The latter is treated in Chapter 4 that considers weakly separable consumer demand. At the end of this chapter the theory about weak separability is extended in order to apply it to linearly homogeneous weakly separable functions and to weakly separable functions with a linearly homogeneous subfunction.

\subsection{Existence of an Aggregator Function}

Suppose a given set of producer data on $X_{1} \times X_{2}$ and a given function $h: X_{2} \rightarrow \mathbb{R}$. Then we may investigate whether there exists a corresponding aggregator function $g_{3}$ so that $g\left(x_{1}, h\left(x_{2}\right)\right)$ rationalizes the data. In the following theorem it is done in similar way as in Chapter 4 for weakly separable consumer behaviour.

Theorem 11.1: Suppose $D=\left\{\left(c_{i}, x^{i}, y_{i}\right)\right\}_{i \in l}$ is a finite data set, where $c_{1}: X_{1} \times X_{2} \rightarrow \mathbb{R}_{4}$. Suppose $h: X_{2} \rightarrow \mathbb{R}$ and let the transformation $T_{h}$ of any function $f: X_{1} \times X_{2} \rightarrow \mathbb{R}$ be defined by

$$
\left(T_{h} f\right)\left(x_{1}, x_{2}\right)=\inf _{h(v) \geq h\left(x_{2}\right)} f\left(x_{1}, w\right) \quad\left(x \in X_{1} \times X_{2}\right) .
$$

Suppose $\left(\boldsymbol{T}_{h} c_{i}\right)\left(x^{i}\right)=c_{i}\left(x^{i}\right)$, for all $i \in I$, and $p u t D^{\prime}=\left\{\left(\boldsymbol{T}_{h} c_{i}, x^{i}, y_{i}\right)\right\}_{i \in I^{-}}$.

(a) Then one has $\mathcal{F}_{d}^{o}\left(D^{\prime}\right) \subset \mathcal{F}_{d}^{\circ}(D)$.

(b) If $\mathcal{F}_{d}^{o}\left(D^{\prime}\right) \neq \varnothing$ then there exists a function $f \in \mathcal{F}_{d}^{o}(D)$ such that $T_{h} f=f$.

(c) Suppose $T_{h} f=f$ and suppose that the infima for each transformation $T_{h} c_{i}$ are attained, $i . e$. for every $i \in l$ and $x \in X$ there is $a$ ve $X_{2}$ such that

$$
\left(T_{h} c_{i}\right)(x)=c_{i}\left(x_{1}, v\right), h(v) \geq h\left(x_{2}\right) \text {. }
$$

Then $f \in \mathcal{F}_{d}^{0}(D)$ if and only if $f \in \mathcal{F}_{d}^{o}\left(D^{\prime}\right)$.

Proof (a): By Theorem $4.6(a)$ one has $\mathcal{F}_{d}\left(D^{\prime}\right) \subset \mathcal{F}_{d}(D)$. Then we have obviously also $\mathcal{F}_{d}^{d}\left(D^{\prime}\right) \subset \mathcal{F}_{d}^{d}(D)$.

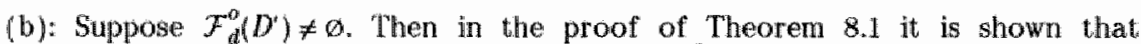
there exists anotonic transformation $m$ of $\in \mathcal{F}_{d}^{a}\left(D^{\prime}\right)$ of a function of the form

$$
f(x)=\min _{i=I} f_{i}+\lambda_{i}\left[\left(T_{h} c_{i}\right)(x)-\left(T_{h}^{n} c_{i}\right)\left(x^{i}\right)\right] \quad\left(x \in X_{1} \times X_{2}\right)
$$

where $\lambda_{i}>0$ and $f\left(x^{i}\right)=f_{i}$ for all $i \in I$. This function satisfies obviously $T_{h} f=f$, so that also $T_{h}($ mof $)=$ mof, because $n$ is monotonically increasing. Furthermore, by (a) one has $\mathcal{F}_{d}^{o}\left(D^{*}\right) \subset \mathcal{F}_{d}^{d}(D)$ and thus mof $\in \mathcal{F}_{d}^{0}(D)$.

(c): By Theorem $4.6(c)$ we have $f \in \mathcal{F}_{d}(D)$ if and only if $f \in \mathcal{F}_{d f}\left(D^{r}\right)$. Hence, this is obviously also the case when output $y_{i}$ is observed.

As it was the case for Theorem 4.6 , the above theorem is also valid if $T_{h}$ is defined as

$$
\left(T_{h} f\right)\left(x_{1}, x_{2}\right)=\inf _{h(v)=h\left(x_{2}\right)} f\left(x_{1}, v\right)
$$




\subsection{Existence of a Weakly Separable Function}

The existence of a weakly separable production function for producer demand can be easily tested. All we have to test is the existence of a preorder given in the following theorem.

Theorem 11.2: Suppose $D=\left\{\left(c_{i}, x^{i}, y_{1}\right)\right.$ le I is a finte data set of producer behaviout, where $c_{i}: X_{1} \times X_{2} \rightarrow \mathbb{R}_{4}$. Consider the following condations:

(i) There exists a function $g\left(x_{1}, h\left(x_{2}\right)\right) \in \mathcal{F}_{d}^{-}(D)$, which is weakly separable sin $X_{2}$ and such that $g\left(x_{1}, \eta\right)$ is thcreasing in $\eta$.

(ii) There exists function $g\left(x_{1}, h\left(x_{2}\right)\right) \in \mathcal{F}_{d}^{\circ}(D)$, which is wedkly separable on $X_{2}$ and such that $g\left(x_{1}, \eta\right)$ is strictly increasing in $\eta$.

(iii) There exists a prearder $-\infty$ on $\left\{x_{2}^{i}\right\}_{\text {iel }}$ such that

$$
\begin{array}{lll}
c_{i}\left(x_{1}^{j}, x_{2}^{k}\right) \leq c_{i}\left(x^{i}\right), y_{j}>y_{i} \Rightarrow x_{2}^{k}<x_{2}^{j} & (i, j, k \in I), \\
c_{i}\left(x_{1}^{j}, x_{2}^{k}\right)<c_{i}\left(x^{i}\right), y_{j} \geq y_{i} \Rightarrow x_{2}^{k}<x_{2}^{j} & (i, j, k \in I), \\
c_{i}\left(x_{1}^{j}, x_{2}^{k}\right) \leq c_{i}\left(x^{i}\right), y_{j} \geq y_{i} \Rightarrow x_{2}^{k} \preccurlyeq x_{2}^{j} & (i, j, k \in I) .
\end{array}
$$

(iv) There exists a weakly separable function $g\left(x_{1}, h\left(x_{2}\right)\right) \in \mathcal{F}_{d}^{o}(D)$, such that $h$ and $g$ are strictly monotonically increasing continuous functions, and furthermore $h$ is concave and $g\left(*, h\left(x_{2}\right)\right)$, for every fixed $x_{2} \in X_{2}$, guast-concave.

Then:

(a) (ii) $\Rightarrow$ (iii) $\Rightarrow$ (i).

(b) Suppose every function

$$
h\left(x_{2}\right)=\min _{i, j \in l} \alpha_{i j}+\lambda_{i} c_{i}\left(x_{1}^{j}, x_{2}\right) \quad\left(x_{2} \in X_{2}\right)
$$

where $\alpha_{i j} \in \mathbb{R}$ and $\lambda_{i}>0$, is such that the functions

$$
\vec{c}_{i}\left(x_{1}, \eta\right)=\inf _{h\left(x_{2}\right) \geq \eta} c_{i}\left(x_{1}, x_{2}\right) \quad\left(i \in I, x_{1} \in X_{11}, \eta \in \mathbb{R}\right),
$$

are strictly increasing in 7 . Then (iil) $\leftrightarrow$ (ii).

(c) Suppose $D$ is a price and quantity data set. Then (ii) $\Leftrightarrow$ (ii) $\Leftrightarrow$ (iv).

Proof (a) (ii) $\Rightarrow$ (iii): Suppose $f(x)=g\left(x_{1}, h\left(x_{2}\right)\right) \in \mathcal{F}_{d,}^{o}(D)$ is weakly separable and $g\left(x_{1}, \eta\right)$ is strictly increasing in $\eta$. Let $>_{\eta} \sim$ be the weak order induced by the subfunction $h$ on $\left\{x_{2}^{i}\right\}_{i e f}$ and let $i, j, k \in I$. Then one has

$$
\begin{aligned}
c_{i}\left(x_{1}^{j}, x_{2}^{k}\right) \leq c_{i}\left(x^{i}\right), y_{j}>y_{i} & \Rightarrow g\left(x_{1}^{j}, h\left(x_{2}^{k}\right)\right) \leq y_{i}<y_{j}=g\left(x_{1}^{j}, h\left(x_{2}^{j}\right)\right) \\
& \Rightarrow h\left(x_{2}^{k}\right)<h\left(x_{2}^{j}\right) \\
& \Rightarrow x_{2}^{k}<x_{2}^{j} .
\end{aligned}
$$

The other implications can be proved in a similar way.

(iii) $\Rightarrow$ (i): If we rewrite (iii) using

$$
\begin{aligned}
& y_{i} \geq y_{j} \rightarrow x^{i} \succeq x^{j} \quad(i, j \in I), \\
& x_{2}^{i} \geq x_{2}^{j} \rightarrow x_{2}^{i} \searrow^{j} \quad(i, j \in I),
\end{aligned}
$$

then the preorders $\succ, \sim$ and $\succ^{\prime}, \sim$ satisfy the condition given in Theorem 4.7 (iii). Thus, as is shown in the proof of that theorem, we can construct a function $h$ such that we have $\left(\boldsymbol{T}_{h} c_{i}\right)\left(x^{i}\right)=c_{i}\left(x^{i}\right)$, for all $i \in \dot{I}$, where

$$
\left(T_{h} c_{i}\right)\left(x_{1}, x_{2}\right)=\inf _{h(v) \geq h\left(x_{2}\right)} c_{i}\left(x_{1}, v\right) \quad\left(i \in I, x \in X_{1} \times X_{2}\right)
$$

Note that the function $h$ in the proof of Theorem 4.7 satisfies 


$$
\begin{aligned}
& \left(T_{h} c_{i}\right)\left(x^{j}\right) \leq\left(T_{h} c_{i}\right)\left(x^{i}\right) \Rightarrow x^{j}<x^{i} \Rightarrow y_{j} \leq y_{i} \quad\left(i_{n} j \in I\right) \\
& \left(T_{h} c_{i}\right)\left(x^{j}\right)<\left(T_{h} c_{i}\right)\left(x^{i}\right) \Rightarrow x^{j}<x^{i} \Rightarrow y_{j}<y_{i} \quad\left(i_{j} \in I\right) .
\end{aligned}
$$

Hence, by liheoren 8.1 we have $F_{d}^{o}\left(D^{*}\right) \neq \emptyset$ for $D^{*}=\left\{\left(T_{h} c_{i}, x^{i}, y_{i}\right)\right\}_{\text {ine }}$. Thus by Theorem 11.L (b) there exists a function $T_{k} f=f \in \mathcal{F}_{d}^{o}(D)$.

(b) (iii) $\Rightarrow$ (ii): Every function $\boldsymbol{T}_{\mathrm{h}} c_{i}$ in the above proof is weakly separable. Now, the assumption in (b) implies that its aggregator function $\vec{c}_{i}\left(x_{1} \eta\right)$ is strictly increasing in $\eta$. In that case the constructed weakly separable function $g\left(x_{1}, h\left(x_{2}\right)\right) \in \mathcal{F}_{d}^{\circ}(D)$ in the proof of Theorem 11.1 (b) will also have these properties.

(c) One can prove that the assimption made in (b) is satisfied by price and quantity data. Theorem 4.8 contains a complete proof for the interested reader.

To prove (iii) $\Rightarrow$ (iv) we may construct functions $g$ and $h$ as in the proof of (b). When we use a continuous monotonic transformation $m$, these functions will have the mentioned ptoperties, because they are derived from price and quantity data. Finally, the implication (iv) $\Rightarrow$ (ii) is trivial.

I used condition (iii) of the above theorem to test whether goods and services of the Dutch industry data are weakly separable, assuming the aggregator function $g\left(x_{1}, \eta\right)$ is strictly increasing in $\eta$. Only two observation yearis had to be removed in order to accept this hypothesis. The theorem does provide an estimation of the economical efficiency upper bound for the efficiency transformation of Example 2.3. By Theorem 11.2 (a), condition (iii) is a necessary condition for the transformed data to be weakly separable. So we may use this condition in order to compute an upper bound of the economicall efficiency. The result of this computation was $e=99.5 \%$.

\subsection{Existence of a Linearly Homogeneous Subfunction}

The nonparametric restrictions for weakly separable functions with a linearly homogeneous subfunction $h$ follow from the observation that we have

$$
h\left(h\left(x_{2}^{i}\right) h\left(x_{2}^{j}\right)^{-1} x_{2}^{j}\right)=h\left(x_{2}^{i}\right) h\left(x_{2}^{j}\right)^{-1} h\left(x_{2}^{j}\right)=h\left(x_{2}^{i}\right) \quad(i, j \in I) .
$$

This observation is the basis of the following theorem, where the numbers $h_{\text {i }}$ represent the values $h\left(x_{2}^{i}\right)$ :

Theorem 11.3: Suppose $D=\left\{\left(c_{i}, x^{i}, y_{i}\right)\right\}_{i \in I}$ is a finite data set of producer behaviour, where $c_{i}: X_{1} \times X_{2} \rightarrow \mathbb{R}_{+}$and $X_{2}$ is a cone. Consider the fallowing conditions:

(i) There exists a weakly separable fanction $g\left(x_{1}, h\left(x_{2}\right)\right) \in \mathcal{X}^{\text {id }}(D)$ such that the subfunction $h: X_{2} \rightarrow \mathbb{R}$ is linearly homogeneans and the aggregator function $g\left(x_{1}, \eta\right)$ is increasing in $\eta$.

(ii) There exist numbers $h_{i}>0$ such that

$$
\begin{aligned}
& c_{i}\left(x_{1}^{i}, h_{i} h_{j}^{-1} x_{2}^{j}\right) \geq c_{i}\left(x^{i}\right) \quad(i, j \in I), \\
& y_{j} \geq y_{i} \Rightarrow c_{i}\left(x_{1}^{j}, h_{j} h_{i}^{-1} x_{2}^{i}\right) \geq c_{i}\left(x^{i}\right) \quad(i, j \in I), \\
& y_{j}>y_{i} \Rightarrow c_{i}\left(x_{1}^{j}, h_{j} h_{i}^{-1} x_{2}^{i}\right)>c_{i}\left(x^{i}\right) \quad(i, j \in I) .
\end{aligned}
$$

Then:

(a) Suppose $c_{i}\left(x_{1}^{i}, 0\right)<c_{i}\left(x^{i}\right)$ for all $i \in I$. Then $(i) \Rightarrow$ (ii).

(b) Suppose all cost functions

$$
c_{i}(x)=\alpha_{i}\left(x_{1}, \beta_{i}\left(x_{2}\right)\right) \quad\left(x \in X_{1} \times X_{2}, i \in I\right)
$$

are weakly separable in $X_{2}$, where $\beta_{i}: X_{2} \rightarrow \mathbb{R}_{+}$are linearly homogeneous, $\beta_{i}\left(x_{2}^{i}\right)>0$, and $\alpha_{i}\left(x_{1}, \beta\right)$ increasing in $\beta$, for all $i \in I$. Then (ii) $\Rightarrow$ (i). 
Proof (a): Put $h_{i}=h\left(x_{2}\right)$ for all $i \in I$. These values $h_{i}$ are positive, because one has

$$
c_{i}\left(x_{1}^{i}, 0\right)<c_{i}\left(x^{i}\right) \Rightarrow g\left(x_{1}^{i}, h(0)\right)<g\left(x_{1}^{i}, h\left(x_{2}^{i}\right)\right) \Rightarrow 0=h(0)<h\left(x_{2}\right) \quad(i \in I) .
$$

Now, the result follows from the definition of producer denand, because $h$ linearly homogeneous implies that we have

$$
g\left(x_{1}^{i}, h\left(h_{i} h_{j}^{-1} x_{2}^{j}\right)\right)=g\left(x_{1}^{i}, h\left(x_{2}^{i}\right)\right), g\left(x_{1}^{j}, h\left(h_{j} h_{i}^{-1} x_{2}^{i} j\right)=g\left(x_{1}^{j}, h\left(x_{2}^{j}\right)\right) \quad(i, j \in l) .\right.
$$

(b): Puti

$$
h\left(x_{2}\right)=\inf _{i \in I} h_{i} \beta_{i}\left(x_{2}\right) / \beta_{i}\left(x_{2}^{i}\right) \quad\left(x_{2} \in X_{2}\right)
$$

I will show that we can apply Theorem 11:1(b) to this function. From the assumption

$$
c_{i}\left(x_{1}^{i}, h_{i} h_{j}^{-1} x_{2}^{j}\right) \geq c_{i}\left(x^{i}\right) \quad(i, j \in I)
$$

and the form of the cost functions, we obtain

$$
h_{i} h_{j}^{-1} \beta_{i}\left(x_{2}^{j}\right) \geq \beta_{i}\left(x_{2}^{i}\right) \quad\left(i_{i} j \in I\right) \text {. }
$$

Hence, we have $h\left(x_{2}^{i}\right)=h_{i}$ and thus

$$
h(v) \geq h\left(x_{2}^{i}\right) \Rightarrow \beta_{i}(v) \geq \beta_{i}\left(x_{2}^{i}\right) \Rightarrow c_{i}\left(x_{1}^{i}, v\right) \geq c_{i}\left(x^{i}\right) \quad\left(i \in I, v \in X_{2}\right)
$$

The latter implies $\left(\boldsymbol{T}_{h} c_{i}\right)\left(x^{i}\right)=c_{i}\left(x^{i}\right)$, for all $i \in I$, in Theorem 11.1. Furthermore, from the assumption

$$
\begin{array}{ll}
y_{j} \geq y_{i} \Rightarrow c_{i}\left(x_{1}^{j}, h_{j}, h_{i}^{-1} x_{2}^{i}\right) \geq c_{i}\left(x^{i}\right) & (i, j \in I), \\
y_{j}>y_{i} \Rightarrow c_{i}\left(x_{1}^{j}, h_{j}, h_{i}^{-1} x_{2}^{i}\right)>c_{i}\left(x^{i}\right) & (i, j \in I),
\end{array}
$$

and Theorem 8.1 we obtain $\mathcal{F}_{d}^{o}\left(D^{\prime}\right) \neq \emptyset$ for $D^{\prime}=\left\{\left(\boldsymbol{T}_{h} c_{i}, x^{i}, y_{i}\right)\right\}_{\text {ier }}$. Hence the result follows from Theorem 11.1 (b).

The following theorem provides conditions, which may be tested in a computational simple way.

Theorem 11.4: Suppose $D=\left\{\left(c_{i}, x^{i}, y_{i}\right)\right\}_{i \in I}$ is a finite data set of producer behaviour, where $c_{i}: X_{1} \times X_{2} \rightarrow \mathbb{R}_{+}$and $c_{i}\left(x^{i}\right)>0$, for all $i \in I$, and $X_{2}$ is a cone. Suppose alh functions $c_{i}$ ore of the form

$$
c_{i}(x)=\alpha_{i}\left(x_{1}\right)+\beta_{i}\left(x_{2}\right) \quad\left(i \in I, x \in X_{1} \times X_{2}\right),
$$

where $\beta_{i}: X_{2} \rightarrow \mathbb{R}_{+}$is linearly homogeneous and $\beta_{i}\left(x_{2}^{i}\right)>0$, for all $i \in \mathbb{I}$. Then the following conditions are equivalent:

(i) There exists a weakly separable function $g\left(x_{1}, h\left(x_{2}\right)\right) \in \mathcal{F}_{d}(D)$ such that the subfunction $h: X_{2} \rightarrow \mathbb{R}$ is linearly homogeneows and $g\left(x_{1}, \eta\right)$ is increasing in $\eta$.

(ii) There exist values $\phi_{i}=\ln h\left(x_{2}^{i}\right)$ such that

$$
\phi_{i}-\phi_{j} \leq \min \left\{a_{i j}, b_{i j}\right\}, \phi_{i}-\phi_{j}<c_{i j} \quad(i, j \in I)
$$

where

$$
\begin{aligned}
& a_{i j}=\ln \left(\beta_{j}\left(x_{2}^{i}\right) / \beta_{j}\left(x_{2}^{j}\right)\right) \quad(i, j \in I), \\
& b_{i j}= \begin{cases}\ln \left(\beta_{i}\left(x_{2}^{i}\right) /\left[c_{i}\left(x^{i}\right)-\alpha_{i}\left(x_{1}^{j}\right)\right]\right) & \left(i, j \in I: y_{j} \geq y_{i}, c_{i}\left(x^{i}\right)>\alpha_{i}\left(x_{1}^{j}\right)\right), \\
\infty & \text { (atherwise). }\end{cases} \\
& c_{i j}= \begin{cases}\ln \left(\beta_{i}\left(x_{2}^{i}\right) /\left[c_{i}\left(x^{i}\right)-\alpha_{i}\left(x_{1}^{j}\right)\right]\right) & \left(i, j \in I: y_{j}>y_{i}, c_{i}\left(x^{i}\right)>\alpha_{i}\left(x_{1}^{j}\right)\right), \\
\infty & \text { (otherwise). }\end{cases}
\end{aligned}
$$

Proof: This follows by applying Theorem 11.3. Note that the assumptions in Theorem 11.3 (a) and (b) are satisfied, and we have thus (i) $\Leftrightarrow$ (ii) in that theorem. Further, if we write out the conditions on the numbers $h_{i}$ in 
Theorem 11.3 (ii), we obtain as necessary and sufficient conditions

$$
\ln h_{i}-\ln h_{j} \leq \text { min }\left\{a_{i j}, b_{i j}\right\}_{*}, \ln h_{i j}-\ln h_{j}<c_{i j} \quad(i, j \in I) .
$$

Note that the weakly separable function in the above theorem is a generalization of a homothetic function, which was treated in the previous chapter. A homothetic function can be conceived as a weakly separable function $g(h(x))$ with a linearly homogeneous subfunction $h(x)$. In that case one has $X_{1}=\emptyset$ and $x=x_{2}$, and we may take $\alpha_{i}=0$ in the above theorem. Condition (ii) reduces then to condition (1ii) of Theorem 10.1, which is a necessary and sufficient condition for the bypothesis of homothetic producer demand.

Application of Theorem 11.3, in order to test the hypothesis of linearly homogeneous weak separability of goods and services in the Dutch industry data, resulted in the elimination of seven observations.

\subsection{Existence of a Linearly Homogeneous Weakly Separable Function}

The above theory can be extended to test the hypothesis that the weakly separable function is linearly homogeneous. Then we need as an additional assumption that the cost functions are linearly homogeneous. For the derivation of the results II refer the reader to the beginning of Chapter 9 , where the transformation

$$
\left(c_{i}, x^{i}, y_{i}\right) \rightarrow\left(c_{i}, y_{i}^{-1} x^{i}, 1\right) \quad(i \in I)
$$

is proposed. As argued in Chapter 9, this scalling of the data makes the assumption that the production function is linearly homogeneous in the nonparametric test superfluous. This is also the case when linearly homogeneous weak separability is considered. The following results include data sets of an infinite number of observations, which was not the case in the earlier results concerning separability.

Theorem 11.5: Suppose $D=\left\{\left(c_{i}, x^{i}, y_{i}\right)\right\}_{i \in I}$ is a data set of producer behaviour, where $c_{i}: X_{1} \times X_{2} \rightarrow \mathbb{R}_{+}$are timearly homogeneous, $c_{i}\left(x^{i}\right)>0$ and $y_{i}>0$, for all $\dot{x} \in I$, and $X_{1}$ and $X_{2}$ are cones. Put

$$
v_{i}=y_{i}^{-1} x^{i} \quad(i \in I) \text {. }
$$

Consider the following conditions:

(i) There exists a linearly homogeneous weakly separable function $g\left(x_{1}, h\left(x_{2}\right)\right) \in \mathcal{F}_{d}^{0}(D)$ such that the subfunction $h: X_{2} \rightarrow \mathbb{R}$ is linearly homogeneous and $g\left(x_{1}, \eta\right)$ is increasing in $\eta$.

(ii) There exist numbers $h_{i}>0$ such that

$$
c_{i}\left(v_{1}^{i}, h_{i} h_{j}^{-1} v_{2}^{j}\right) \geq c_{i}\left(v^{i}\right), c_{i}\left(v_{i}^{j}, h_{j} h_{i}^{-1} v_{2}^{i}\right) \geq c_{i}\left(v^{i}\right) \quad(u, j \in I) .
$$

Then:

(a) Suppose $c_{i}\left(v_{1}^{i}, 0\right)<c_{i}\left(v^{i}\right)$ for all $i \in I$. Then (i) $\Rightarrow$ (ii).

(b) Suppose all cost functions

$$
c_{i}(x)=\alpha_{i}\left(x_{1}, \beta_{i}\left(x_{2}\right)\right) \quad\left(x \in X_{1 \times} X_{2}, i \in I\right)
$$

are weakly separable, where the functions $\beta_{i}: X_{2} \rightarrow \mathbb{R}_{+}$are limearly homogeneous, $\beta_{i}\left(v_{2}^{i}\right)>0$, and $\alpha_{i}\left(x_{1}, \beta\right)$ is increasing in $\beta$, for all $i \in I$. Then (ii) $\Rightarrow$ (i).

Proof (a): In case of finite data sets, we may apply Theorem 11.3(a) to the scaled data $\left\{\left(c_{0}, v, 1\right)\right\}_{\text {ier }}$, because the hypothesis of linearly homogeneons producer demand is inwariant under scaling. One can check that the proof of Theorem 11.3 (a) remains valid in case infinite data sets are used.

(b): Put

$$
h\left(x_{2}\right)=\inf _{i \in I} h_{i} \beta_{i}\left(x_{2}\right) / \beta_{i}\left(x_{2}^{i}\right) \quad\left(x_{2} \in X_{2}\right)
$$




$$
f(x)=g\left(x_{1}, h\left(x_{2}\right)\right)=\inf _{i \in I} c_{i}\left(x_{1}, h_{i}^{-1} h\left(x_{2}\right) \beta_{i}\left(v_{2}^{i}\right)\right) / c_{i}\left(v^{i}\right) \quad(x \in X) .
$$

Becanse the hypothesis of linearly homogeneous producer demand is invariant under scaling, it is sufficient to show that we have $f \in f_{d}^{0}\left(1\left(c_{w}, w^{i}, 1\right) f_{i \in f}\right)$. First, $\mathbb{I}$ will show that all the output values $f\left(v^{i}\right)$ are equal to one. From

$$
c_{i}\left(v_{1}^{i}, h_{i} h_{j}^{-1} v_{2}^{\gamma}\right) \geq c_{i}\left(v^{i}\right) \quad(i, j \in I)
$$

in (ii), and the assumptions about the form of the cost functions we obtain

$$
h_{i} h_{j}^{-1} \beta_{i}\left(v_{2}^{j}\right) \geq \beta_{i}\left(w_{2}^{i}\right) \quad(i, j \in I)
$$

which in turn means

$$
h\left(v_{2}^{j}\right)=\inf _{i \in I} h_{j} \beta_{i}\left(v_{2}^{j}\right) / \beta_{i}\left(v_{2}^{i}\right)=h_{j} \quad(j \in I) .
$$

This and

$$
c_{i}\left(v_{1}^{j}, h_{j} h_{i}^{-1} w_{2}^{i}\right) \geq c_{i}\left(v^{i}\right) \quad(i, j \in I)
$$

in (ii), imply

$$
g\left(v_{1}^{j}, h\left(v_{2}^{j}\right)\right)=\inf _{i \in I} c_{i}\left(x_{1}, h_{i}^{-1} h_{j} \beta_{i}\left(v_{2}^{i}\right)\right) / c_{i}\left(v^{i}\right)=1 .
$$

So the output values are correct. It remains to show that $f$ may generate the scalled producer demand data. Erom

$$
h\left(x_{2}\right) \leq h_{i} \beta_{i}\left(x_{2}\right) / \beta_{i}\left(y_{2}^{i}\right) \quad\left(x_{2} \in X_{2}, i \in I\right) \text {, }
$$

and the weak separability of the cost functions, we obtain

$$
c_{i}(x) \leq c_{i}\left(x_{1}, h_{i}^{-1} h\left(x_{2}\right) \beta_{i}\left(v_{2}^{i}\right)\right) \quad(x \in X, i \in I) .
$$

So the construction of $f$ implies

$$
f(x) \leq c_{i}\left(x_{1}, h_{i}^{-1} h\left(x_{2}\right) \beta_{i}\left(v_{2}^{i}\right)\right) / c_{i}\left(v^{i}\right) \leq c_{i}(x) / c_{i}\left(v^{i}\right) \quad(x \in X, \quad i \in I) .
$$

Hence

$$
c_{i}(x) \leq c_{i}\left(v^{i}\right) \Rightarrow f(x) \leq 1, c_{i}(x)<c_{i}\left(w^{i}\right) \Rightarrow f(x)<1 \quad(x \in X, i \in I),
$$

thus $f$ is a rationalizing linearly homogeneous weakly separable function.

Remark: I have to make a remark about the form of the constructed function $f$ in the above proof of Theorem $11.5(\mathrm{~b})$, which is not an arbitracy one. In the theory concerning linearly homogeneous functions it is often possible to construct a suitable rationalizing function by taking the least upper bound over a set of rationalizing linearly homogeneous functions. This applies to this case as well. In the proof of Theorem 11.5 (b) we have $f(x)=U(F, x)$, where $F$ is the subset of all linearly homogeneous functions in

$$
\left\{g\left(x_{1}, h\left(x_{2}\right)\right\} \in \mathcal{F}_{d}^{o}(D), h \text { linearly homogeneous, } h\left(x_{z}^{i}\right)=h_{i} \text { for all } z \in I\right\} \text {. }
$$

In this particular case this function is of the form

where

$$
U(F, x)=\inf _{i \in I} \alpha_{i}\left(x_{1}, U\left(H, x_{2}\right) h_{i}^{-1} \beta_{i}\left(v_{2}^{i}\right)\right) / c_{i}\left(v^{i}\right) \quad(x \in X)
$$

$$
\begin{aligned}
& H=\left\{h \in \mathcal{F}_{d}^{o}\left(\left\{\left(\beta_{i}, v_{2}^{i}, h_{i}\right)\right\}_{i \in I}\right) / h \text { linearly homogeneous }\right\} \\
& U\left(H, x_{2}\right)=\inf _{i \in I} h_{i} \beta_{i}\left(x_{2}\right) / \beta_{i}\left(v_{2}^{i}\right) \quad(x \in X\} .
\end{aligned}
$$

It is left to the reader to verify these statements.

Just like in Theorem 11.4, which provides computational simple conditions for the test in Theorem 11.3, we can derive such a theorem for the test in the above theorem. 
Theorem 11.6: Suppose $D=\left\{\left(c_{i}, x^{i}, y_{i}\right)\right\}_{\text {iel }}$ is a data set of producer behaviour, where $c_{i}, X_{1} \times X_{2} \rightarrow R$ and $y_{1}>0$, for all $i \in I$, and $X_{1}$ and $X_{2}$ are cones. Suppose all functions $c_{1}$ are of the form

$$
c_{i}(x)=\alpha_{i}\left(x_{1}\right)+\beta_{i}\left(x_{2}\right) \quad\left(x \in X_{1} x_{1} X_{2}, i \in I\right)_{3}
$$

where both $\alpha_{i} ; X_{1} \rightarrow \mathbb{R}_{+}$and $\beta_{i}: X_{2} \rightarrow \mathbb{R}_{4}$ are linearly homogeneous and $\beta_{i}\left(x_{2}\right)>0$. Put $v_{i}=y_{i}^{-1} x^{i}$ for all $i \in I$. Then the following conditions are equwalent:

(i) There exists a linearly homogeneous weakly separable function $g\left(x_{1}, h\left(x_{2}\right)\right) \in \mathcal{X}_{d}^{0}(D)$ such that the subfunction $h: X_{2} \rightarrow \mathbb{R}$ is linearly homogeneous and $g\left(x_{1}, \eta\right)$ is increasing in $\eta$.

(ii) There exist values $\phi_{i}=\ln h\left(x_{2}^{i}\right)$ such that

$$
\phi_{i}-\phi_{j} \leq \min \left\{a_{i j}, b_{i j}\right\} \quad\left(i_{i} j \in I\right)_{3}
$$

where

$$
\begin{aligned}
& a_{i j}=\ln \left(\beta_{j}\left(v_{2}^{i}\right) / \beta_{j}\left(v_{2}^{j}\right)\right) \quad(i, j \in I), \\
& b_{i j}=\left\{\begin{array}{l}
\ln \left(\beta_{i}\left(v_{2}^{i}\right) /\left[c_{i}\left(v^{i}\right)-\alpha_{i}\left(v_{1}^{j}\right)\right]\right) \\
\infty
\end{array}\right.
\end{aligned}
$$$$
\left(i, j \in I: c_{i}\left(v^{i}\right)-\alpha_{i}\left(v_{1}^{j}\right)>0\right)
$$

(otherwise).

Proof: The result follows by applying Theorem 11.5. Note that the assumptions in Theorem 11.5 (a) and (b) are satisfied. By writing out the conditions on the numbers $h_{i}$ in Theorem 11.5 (ii), we obtain (ii) as a necessary and sufficient condition for (i).

Remark: The matrix $d_{i j}=\min \left\{a_{i j}, b_{i j}\right\}$ in Theorem 11.6 has an interpretation which concerns the relative upper bounds of subfunction $h$. As the reader may verify, one has

$$
U_{/}\left(H, x_{1}^{i}, x_{2}^{j}\right)=\mathrm{e}^{d_{i j}^{*}} \quad(i, j \in \Pi)
$$

where $H$ contains all linearly homogeneous functions $h: X_{2} \rightarrow \mathbb{R}$ such that there exists a linearly homogeneous function $g\left(x_{1}, h\left(x_{2}\right)\right) \in \mathcal{F}_{d}^{o}(D)$.

I applied condition (ii) of Theorem 11.6 to test the hypothesis of linearly homogeneous weak separability of goods and services for the Dutch industry data. The results were the same as obtained earlier, where no restriction on the aggregator function was supposed. A subset of only two observations is found to be consistent with this hypothesis.

Now, let us consider the existence of a linearly homogeneous weakly separable function $g\left(x_{1}, h\left(x_{2}\right)\right)$ for which the subfunction $h$ is not necessarily linearly homogeneous: In that case there is no simple way to construct a rationalizing production function. However, by scaling the data, we can obtain the following result from Theorem 11.2

Theorem 11.7: Suppase $D=\left\{\left(c_{i}, x^{i}, y_{i}\right)\right\}_{i \in \mathbb{R}}$ is a finite data set of producer behawour, where $c_{i}: X_{1} \times X_{2} \rightarrow \mathbb{R}_{+}$are linearly homogeneous, $c_{i}\left(x^{i}\right)>0$, and $y_{1}>0$, for wll $\in \mathbb{L}$, and $X_{1} \times X_{2}$ is a cone. Consider the following condition.

(i) There exists a preorder $>, \sim$ on $\left\{v_{2}^{\text {i }}\right\}_{\text {ieI }}$ such that

$$
\begin{array}{ll}
c_{i}\left(v_{1}^{j}, v_{2}^{k}\right)<c_{i}\left(v^{i}\right) \Rightarrow w_{2}^{k}<v_{2}^{j} & \left(i_{1} j, k \in I\right), \\
c_{i}\left(v_{1}^{j}, v_{2}^{k}\right) \leq c_{i}\left(v^{i}\right) \Rightarrow v_{2}^{k}<v_{2}^{j} & \left(i_{j} j, k \in I\right),
\end{array}
$$

where $v^{i}=y_{i}^{-1} x^{i}$ for all $i \in I$.

Put $D^{x}=\left\{\left(c_{i}, u^{t}, 1\right)\right\}_{i \in I \text { * Then: }}$

(a) If there is a linearly bomogeneous function $g\left(x_{1}, h\left(x_{2}\right)\right) \in \mathcal{F}_{d}^{\circ}(D)$, which is weakly separable in $X_{2}$ and such that $g\left(x_{1}, \eta\right)$ is strictly increasing in $\eta$, then (i) is satisfied.

(b) If (i) is satisfied then there exists a function $g\left(x_{1}, h\left(x_{2}\right)\right) \in \mathcal{F}_{d}^{o}\left(D^{2}\right)$, 
which is weakly separable on $X_{2}$.

Proof (a): Suppose the linearly homogeneous function $g\left(x_{1}, h\left(x_{2}\right)\right) \in \mathcal{F}^{-}(D)$ has the properties mentioned in (a). Then this function is also compatible with the scaled data $D^{\prime}$. Hence, $D^{\prime}$ must satisfy condition (iii) in Theorem 11.2 , from which we obtain condition (i).

(b): Suppose (i) is satisfied. Then condition (iii) in Theorem 11.2 holds for the data $D^{*}$. Hence, this theorem implies the existience of the desired weakly separable function.

The problem in this case is the following. If we do not suppose that $h$ is a linearly homogeneous function then the transformed cost functions $\boldsymbol{T}_{h} c_{i}$ in Theorem 11.1 are not necessarily linearly homogeneous, even if we suppose all $c_{i}$ are linearly homogeneous. Hence, the functions $\boldsymbol{T}_{h} c_{i}$ are not suitable to construct a linearly homogeneous weakly separable function. 


\section{INTRODUCTION}

\subsection{Textbook Theory}

In the neoclassical theory the behaviour of a profit maximizing producer is described in the following way. Let $f(\cdot)$ be a production function, $v$ the price of output, and $p$ the price vector of inputs. Profit maximization means that the producer chooses a quantity vector $x^{\prime}$ such that

$$
w f\left(x^{\prime}\right)-p x^{\prime}=\sup _{x} w f(x)-p x \text {. }
$$

This is simple unconditioned optimization without a budget restriction as is illustrated in Figure 12.1.

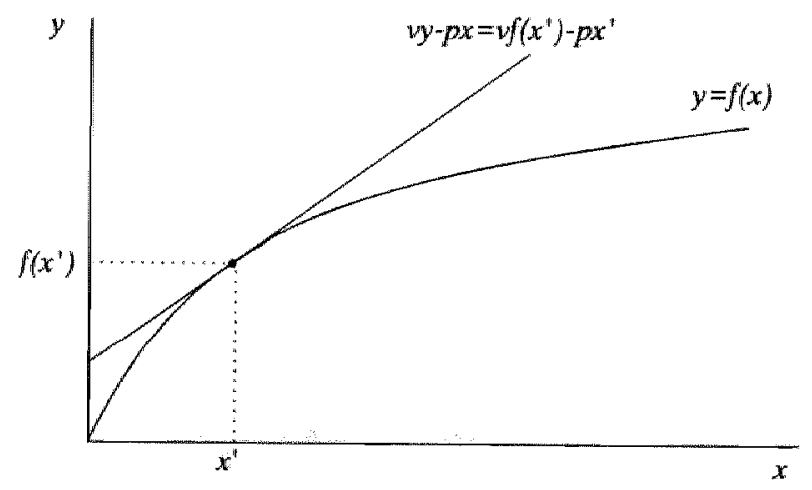

Figure 12.1 Profit Maximization

Profit maximization implies cost minimization, i.e.

$$
p x^{\prime}=\inf _{f(x) \geq f(x)} p x .
$$

Furthermore, profit maximization implies output maximization, i.e.

$$
f\left(x^{n}\right)=\sup _{p x \leq p x^{i}} f(x) \text {. }
$$

Thus producer demand behaviour is a necessary condition for profit maximizing 
behaviour.

\subsection{Profit Maximization}

I will use a more general definition of profit maximizing behaviour. The textbook definition is generalized in a similar way as consumer and producer demand in Part $I$ and $I I$. As in Part II, a production function is assumed to be function $f: X \rightarrow \mathbb{R}$, where $X$ is an arbitary space. Now, suppose the cost of input $x \in X$ is described by a function $c: X \rightarrow \mathbb{R}_{+}$and suppose that $v \in \mathbb{R}_{*}$ is the price of output. Then an input quantity vector $x^{\prime}$, chosen by a profit. maximizing producer, will be such that he maximizes his profit

$$
v f\left(x^{2}\right)-c\left(x^{2}\right)=\max _{x} w f(x)-c(x) \text {. }
$$

The market for output $f(x)$ is thus a competitive market with a fixed price while the market for inputs is not necessarily a competitive market. It may alsio be a monopolistic market.

I assume henceforth $v=1$ without loss of generality, because we can allways rewrite this behaviour as

$$
f\left(x^{\prime}\right)-v^{-1} c\left(x^{\prime}\right)=\max _{x} f(x)-y^{-1} c(x) .
$$

In a similar way as in the definition of producer demand, we obtain the following definition.

Definition: For a function $f: X \rightarrow \mathbb{R}$, the set of profit maximizing behaviour is defined as

$$
D_{p}(f) \equiv\left\{\left(c, x^{\prime}\right) \in C(X) \times X \mid f\left(x^{\prime}\right)-c\left(x^{\prime}\right) \geq f(x)-c(x) \text { for all } x \in X\right\}
$$

Another way to define profit maximizing behaviour is

$$
\left(c, x^{\prime}\right) \in D_{p}(f) \Leftrightarrow f\left(x^{\prime}\right)-c\left(x^{\prime}\right)=\sup _{x} f(x)-c(x)
$$

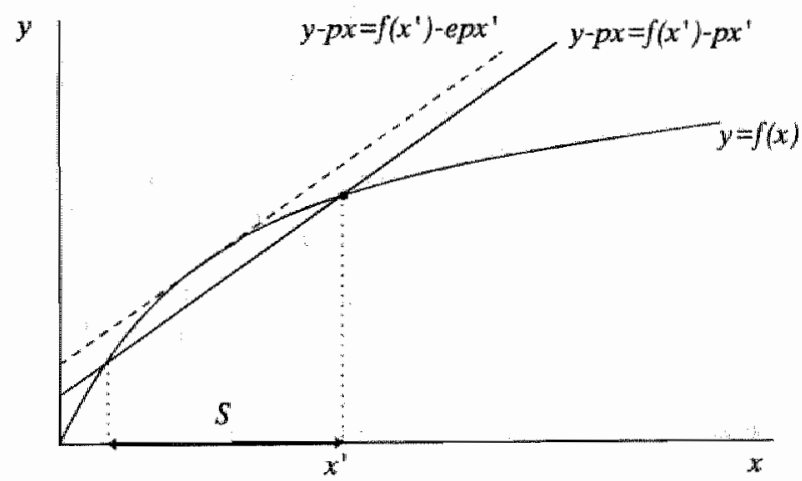

Figure 12.2 Partial Efficient Profit Maximization 
The condition concerning profit maximizing behaviour can also be relaxed by using an efficiency transformation. In fact we may use the same efficiency transformations as we have used for consumer and producer demand. In Figure 12.2 the efficiency transformation of Example 2.3 is used in order to relax the profit maximization conditions on price and quantity data. One has in this figure $\Phi_{e}\left(c, x^{\prime}\right) \in \mathcal{D}_{p}(f)$, where $c$ is of the form $c(x)=p x$. In this figure one has also $\Phi_{f}\left(c, x^{\prime \prime}\right) \in \mathcal{D}_{p}(f)$ for any $x^{\prime \prime} \in S$.

Producer demand is a necessary condition for profit maximization, as is stated in the following theorem.

Theorem 12.1: One has $\mathcal{F}_{p}(D) \subset \mathcal{F}_{d}(D)$ for any data set $D$.

Proof: If one has

$$
f\left(x^{\prime}\right)-c\left(x^{\prime}\right) \geq f(x)-c(x) \quad(x \in X)
$$

then one has also

$$
c(x) \leq c\left(x^{\prime}\right) \Rightarrow f(x) \leq f\left(x^{\prime}\right), c(x)<c\left(x^{\prime}\right) \Rightarrow f(x)<f(x) \quad(x \in X) .
$$

When the observed produced ontput $y=f\left(x^{\prime}\right)$ is contained in the data, we obtain the following slightly different version of the above definition of producer behaviour.

Definition: For a function $f: X \rightarrow \mathbb{R}$, the set of profit maximizing behaviour with obserwed output is defined as

$$
D_{p}^{o}(f) \equiv\left\{\left(c_{i}, x^{\prime}, y\right) \|\left(c, x^{\prime}\right) \in D_{p}(f), y=f\left(x^{\prime}\right)\right\} \text {. }
$$

Profit maximization for frontier production functions is defined in the same way as producer demand in Chapter 7 .

Definition: For a frontier production function $f: X \rightarrow \mathbb{R}$, the set of profit maximizing behaviour with observed output is defined as

$$
D_{p}^{f}(f) \equiv\left\{\left(c, x^{*}, y\right) \mid\left(c, x^{*}\right) \in D_{p}(f), y \leq f\left(x^{*}\right)\right\} \text {. }
$$

\subsection{Contents of Part III}

Chapter 13 is concerned with the existence of a production function for given producer data. Many variations on this problem, like the observation of produced output, frontier productions functions and technical progress, are considered.

Chapter 14 treats homothetic production functions, which are a monotonic transformation $m$ of a linearly homogeneous function $f$. It is shown that profit maximization can be considered as a two stage process for homothetic functions mof $(x)$. The linearly homogeneous function $f$ behaves as a production function that is consistent with cost minimization, while the monotonic transformation $m$ acts as a profit maximizing production function.

The two stage approach, that is applied to homothetic production functions in. Chapter 14, is applied to weakly separable production functions in Chapter 15. Concerning weakly separable profit maximizing functions $g\left(x_{1}, h\left(x_{2}\right)\right)$, it is shown that the subfunction $h$ behaves as a cost minimizing production function and the aggregator function $g$ behaves as a profit maximizing production function. 


\section{PROFIT MAXIMIZATION}

\subsection{Introduction}

There are analogies between profit maximization and linearly homogeneous demand. Let $f$ be a production function and $c$ a linearly homogeneous cost function. Then a necessary and sufficient condition for $x^{*}$ to be a solution for linearly homogeneous demand is

$$
\sup _{x \in X} f(x) / c(x)=f\left(x^{*}\right) / c\left(x^{*}\right) \text {. }
$$

A proof can be found in Färe and Primont (1995, Lemma 3.A.5). The above equality can be written in a profit maximizing form as

$$
\sup _{x \in X} f_{0}(x)-c_{0}(x)=f_{0}\left(x^{2}\right)-c_{0}\left(x^{\prime}\right)
$$

where $f_{0}=\ln f$ and $c_{0}=\ln c$. Hence linearly homogeneous demand behaviour cam be interpreted as a special case of profit maximization behaviour. The proofs of the earlier derived theorems, concerning linearly homogeneous demand, are however guite general. In order to derive results concerning profit maximization, many of these results can be carried over.

There is still another analogy. A necessary and sufficient condition for $x^{\prime}$ to be a solution for linearly homogeneous demand is also

$$
\sup _{x \in X} f(x)-f(x) c(x) / c(x)=0
$$

where $f\left(x^{\prime}\right) / c\left(x^{\prime}\right)$ is equal to the Lagrange multiplier $\lambda$ in the Lagrangian $f(x)-\lambda c(x)$. The above equality can be written in a profit maximizing form as

$$
\sup _{x \in X} f(x)-c_{0}(x)=f(x)-c_{0}\left(x^{\prime}\right)
$$

where $c_{0}(x)=f\left(x^{\prime}\right) c(x) / c\left(x^{\prime}\right)$.

So, the theories concerning both linearly homogeneous demand and profit maximizing behaviour, are related to each other, because linearly homogeneous behaviour appears to be a special case of profit maximizing behaviour. Note that the profit maximizing solutions for the above analogies of linearly homogeneous demand are degenerated solutions. In these cases it is not only a point $x^{*}$ that maximizes the profit, but the whole line $\lambda x^{*}, \lambda>0$, maximizes the profit.

\subsection{Conditions for Profit Maximization}

The conditions concerming profit maximization are similar to the conditions for linearly homogeneous producer demand.

Theorem 13.1: Suppose $D=\left\{\left(c_{i}, x^{i}, y_{i}\right)\right\}_{\text {ie } I}$ is a data set, where $c_{i}: X \rightarrow \mathbb{R}_{+}$. Then the following conditions are equivalent:

(ii) $\mathcal{F}_{p}^{o}(D) \neq \emptyset$

(ii) $y_{j} \leq y_{i}+c_{i}\left(x^{j}\right)-c_{i}\left(x^{i}\right) \quad(i, j \in I)$.

Proof: Similar to the proof of Theorem 9.1. To prove the existence of a function $f \in \mathcal{F}_{p}^{0}(D)$, we may use the function

$$
f(x)=\inf _{i \in \mathbb{f}} y_{i}+c_{i}(x)-c_{i}\left(x^{i}\right) \quad(x \in X) \text {. }
$$

Figure 13.1 shows an example of the construction of a profit maximizing 
production function, using four observations of price and quantity data.

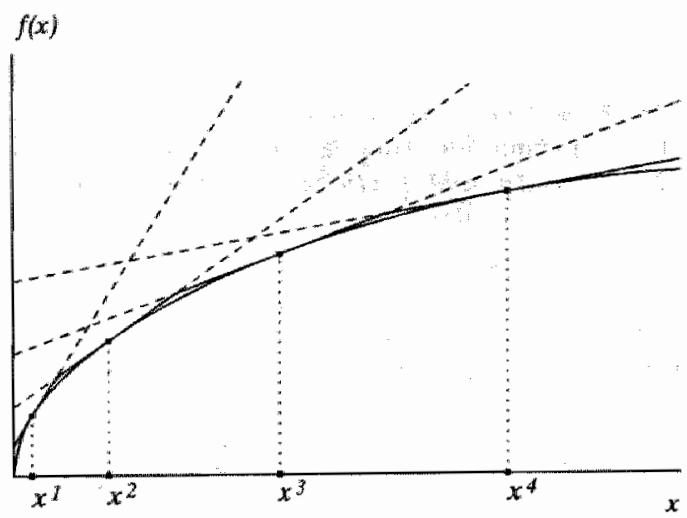

Figure 13.1 Construction Production Function

Application of condition (ii) in Theorem 13.1 to the Dutch industry data resulted in a total rejection of the profit maximization hypothesis. We have to remove all observations, except one, to obtain consistency with economical efficient profit maximization. Note that one observation is always consistent with profit maximization. If we transform the whole data set using the efficiency transformation given in Example 2.3, we obtain as efficiency upper bound $e=81.5 \%$

As is argued above in the introduction of this chapter, there is an interesting analogy between profit maximization and linearly homogeneous cost minimization. The following theorem shows an example of this analogy.

Theorem 13.2: Suppose $D=\left\{\left(c_{i}, x^{i}, y_{i}\right)\right\}_{i \in I}$ is a data set, where all functions $c_{\mathrm{j}}: X \rightarrow \mathbb{R}_{+}$are linearly homogeneous and $X$ is a cone. Put

$$
D^{\prime}=\left\{\left(y_{i} c_{i}(\cdot) / c_{i}\left(x^{i}\right), x^{i}, y_{i}\right)\right\}_{\text {ieit }} D^{\prime \prime}=\left\{\left(\ln c_{i}, x^{i}, \ln y_{i}\right)\right\}_{i \in I} .
$$

Then the following conditions are equivalent:

(i) There exists a linearly homogeneous function in $\mathcal{F}_{d}^{o}(D)$.

(ii) There exists a linearly homogeneous fonction in $\mathcal{F}_{p}^{0}\left(D^{\prime}\right)$.

(iii) $\mathcal{F}_{p}^{o}\left(D^{\prime}\right) \neq 0$

(iv) $F_{p}\left(D^{\prime \prime}\right) \neq 0$.

Proor: The theorem follows from Theorem 13.1 and theorem 9.1, using

$$
\begin{aligned}
& y_{j} \leq y_{i} c_{i}\left(x^{j}\right) / c_{i}\left(x^{i}\right) \Leftrightarrow \\
& y_{j} \leq y_{i}+y_{i} c_{i}\left(x^{i}\right) / c_{i}\left(x^{i}\right)-y_{i} c_{i}\left(x^{i}\right) / c_{i}\left(x^{i}\right) \Leftrightarrow \\
& \ln y_{j} \leq \ln y_{i}+\ln c_{i}\left(x^{j}\right)-\ln c_{i}\left(x^{i}\right) \quad(i, j \in I) .
\end{aligned}
$$

Although only the above theorem is cherived from the analogy of linearly homogeneous cost minimization and profit maximization, there is such an analogy for nearly all the theorems in this chapter. The theoretical 
background of Theorem 13.2 is the following. Linearly homogeneous functions $f \in T^{-8}(D)$ in this theorem satisfy

$$
\begin{aligned}
& \sup _{c_{i}(x) \leq c_{i}\left(x^{i}\right)} f(x)=f\left(x^{i}\right) \Leftrightarrow \\
& \sup _{x \in X} f(x)-f\left(x^{i}\right) c_{i}(x) / c_{i}\left(x^{i}\right)=f\left(x^{i}\right)-f\left(x^{i}\right) c_{i}\left(x^{i}\right) / c_{i}\left(x^{i}\right)=0 \Leftrightarrow \\
& \sup _{x \in X} f(x) / c_{i}\left(x^{i}\right)=f\left(x^{i}\right) / c_{i}\left(x^{i}\right) \quad(i \in I) .
\end{aligned}
$$

\subsection{Bounds for Profit Maximization}

In the theory concerning linearly homogeneous producer demand the relative upper bound was important. Now the following upper bound plays an important role.

Definition: Suppose $F$ is a collection of functions $f: X \rightarrow R$. Then the difference upper bound $U_{-}$is defined as

$$
U_{-}\left(F, x, x^{\prime}\right) \equiv \sup _{f \in F^{\prime}} f(x)-f\left(x^{\prime}\right) \quad\left(x, x^{\prime} \in X\right) .
$$

Similarly, the difference lower bound $L_{-}$is defined as

$$
L_{-}\left(F, x, x^{*}\right) \equiv \inf _{f \in F} f(x)-f\left(x^{*}\right) \quad\left(x, x^{\prime} \in X\right) .
$$

In relation to profit maximization with unobserved output, the theory in Chapter 3, concerning linearly homogeneous consumer demand, is of importance. The chapter started with a restriction on the utility function, using only one observation. On the basis of this a restriction was derived that applies to multiple observations. Concerning profit maximization, I will also derive restrictions from the case where only one observation is available.

Theorem 13.3: Suppose $c: X \rightarrow \mathbb{R}_{+} x^{\prime} \in X$, and put $F=\mathcal{F}_{p}\left(\left\{\left(c, x^{\prime}\right)\right\}\right)$. Then

$$
U_{-}\left(F, x, x^{\prime}\right)=c(x)-c\left(x^{\prime}\right) \quad(x \in X)
$$

Proof: Put:

$$
f(x)=c(x)-c(x):(x \in X)
$$

Then one has clearly

$$
f(x)-c(x)=-c\left(x^{\prime}\right)=f\left(x^{\prime}\right)-c\left(x^{\prime \prime}\right) \quad(x \in X)
$$

Hence $f \in F$. The definition of $U_{-}$implies now the inequality

$$
c(x)-c\left(x^{\prime}\right)=f(x)-f\left(x^{\prime \prime}\right) \leq U_{-}\left(F, x, x^{\prime \prime}\right) \quad(x \in X) \text {. }
$$

To prove the reverse inequality, let $f \in F$. By the definition of profit maximization this is equivalent to

$$
f(x)-f\left(x^{\prime}\right) \leq c(x)-c\left(x^{n}\right) \quad(x \in X) .
$$

Since $f \in F$ was arbitrary, the latter and the definition of $U_{-}$means that we have

$$
U_{-}\left(F, x, x^{\prime}\right) \leq c(x)-c\left(x^{\prime}\right) \quad(x \in X) .
$$

The derived upper bound can be generalized to the casse of several observations. This generalization is valid for even an infinite number of ollservations.

Remark: Although it is in empirical work impossible to have an infinite number of observations, this may be the case in theoretical problems. For example when a data set is described using a continuous index parameter $i$.

As is explained in the introduction of this chapter, there is an analogy 
Wetween linearly homogeneous producer demand and profit maximization. We may obtain profit maximization from linearly homogeneous producer demand by taking the logarithm of the production function and cost function. This analogy implies that we may derive the following theorem, which is similar to Theorem 3.12 in Chapter 3.

Theorem 13.4: Suppose $D=\left\{\left(c_{i}, x^{i}\right)\right\}_{i \in I}$ is a data set, where $c_{i}: X \rightarrow R_{*}$. Put $F=\mathcal{F} p(D)$, and

$$
a_{i j}=c_{i f}(x)-c_{i}\left(x^{i}\right) \quad(i, j \in I) \text {. }
$$

Then:

(a) If $F \neq 0$ then

$$
\begin{aligned}
& U_{-}\left(F_{,}, x^{j}\right) \in \mathcal{F}_{p}(D) \quad(j \in I), \\
& U_{-}\left(F_{,}, x, x^{j}\right)=\inf _{\operatorname{lin}} c_{i}(x)-c_{i}\left(x^{i}\right)+a_{i j}^{*} \quad(x \in X, j \in I), \\
& U_{-}\left(F, x^{i}, x^{j}\right)=a_{i j}^{*} \quad(i, j \in I), \\
& U_{-}\left(F, x^{i}, x^{j}\right)=L_{-}\left(F, x^{j}, x^{i}\right) \quad\left(i_{,} j \in I\right) .
\end{aligned}
$$

(b) $F \neq \emptyset$ if and only if $A$ is absorptive.

Proof (a): This theorem is an analogy of Theorem 3.12, concerning linearly homogeneous consumer demand. While Theorem 3.12 is based on restinictions of the form

$$
\ln U_{N}\left(F^{i}, x^{i}, x^{j}\right) \leq a_{i j}^{*} \quad(i, j \in I)
$$

we have in this case analogous restrictions of the form

$$
U_{-}\left(F, x^{i}, x^{j}\right) \leq a_{i j}^{*} \quad(i, j \in I) .
$$

The proof is completely analogous: The only difference is that we use the function $U_{2}$ instead of In $U_{\%}$. So, for example, instead of

$$
U_{/}\left(F, x^{i}, x^{j}\right)=\mathrm{e}^{a_{i j}^{*}}
$$

in Theorem 3.12 , we write now $U_{-}\left(F, x^{i}, x^{j}\right)=a_{i j}^{*}$.

Note that one has $\ln (a b)=\ln a+\ln b$, hence every multiplication of $U_{f}$ terms in Theorem 3.12 has to be replaced by an addition of $V_{-}$terms.

(b): The proof is similar to the proof of Theorem 3.5, in which we have to replace every multiplication by an addition in the same way as in the proof of $(a)$.

I used Theorem 13.4 (b) to test whether there exists a profit maximizing production function for the Dutch industry data. This condition can be tested using the algorithm given in Appendix $C$, and which is based on Theorem C.4. It appears that eight observations have to be removed in order to get consistency with the hypothesis of profit maximization. The whole data set, translormed using the efficiency transformation given in Example 2.3, has the economical efficiency upper botind $e=99.2 \%$.

The difference with previous test results of profit maximization in this section, leading to much stronger violations, is that the outputs are not avalable. So we may conclude that we do not observe outputs that belong to one and the same production function for all periods. This finding may be an indication for technical inefficiency or technical progress. These properties will be treated later on in this chapter.

When we observe output, the theory of linearly homogeneous producer demand in Chapter 9 is useful. The following theorem corresponds with Theorem 9.2, which treats an upper bound for one observation. 
Theorem 13.5: Sitppose $c: X \rightarrow \mathbb{R}_{+}, x^{*} \in X, y \in \mathbb{R} . P u t F=\mathcal{F}_{p}^{0}(\{(c, x, y)\})$. Then $U(F) \in F^{0}$ and

$$
U(f, x)=y+c(x)-c(x) \quad(x \in X)
$$

Proof: The arguments are similar as in Theorem 9.2 .

Theorem 13.6: Suppose $D=\left\{\left(c_{i}, x^{i}, y_{i}\right)\right\}_{\text {heI }}$ is a data set, where $c_{i}, X \rightarrow \mathbb{R}_{*}, p_{\text {wt }}$ $F=F_{p}(D)$. If $F \neq$ then $U(F, i) \in F$ and

$$
U(F, x)=\inf _{i \in I} y_{i}+c_{i}(x)-c_{i}\left(x^{i}\right) \quad(x \in X) \text {. }
$$

Proof: Similar to the proof of Theorem 9.3 .

\subsection{Frontier Production Functions}

A frontier production function lies above the observed outputs. The minimal possible output values for such a function are interesting. These values are given in the following theorem.

Theorem 13.7: Suppose $D=\left\{\left(c_{i}, x^{i}, y_{i}\right)\right\}_{i \in I}$, where $c_{i}: X \rightarrow \mathbb{R}_{4}$, and $\mathcal{F}_{p}(D) \neq \emptyset$. Then

$$
L\left(\mathcal{F}_{p}^{f}(D), x^{i}\right)=\max _{k \in I} y_{k}+L_{-}\left(\mathcal{F}_{p}(D), x^{i}, x^{k}\right) \quad(\dot{i} \in I) .
$$

Proof: This is analogous to Theorem 9.4, concerning linearly homogeneous producer demand. Note that every multiplication operation in Theorem 9.4 is now represented by an addition. There is the following connection between both theorems

$$
\ln L \rightarrow L, \ln L_{\gamma} \rightarrow L_{-}, \ln y_{k} \rightarrow y_{k}
$$

So we have in the expression of $L$

$$
\max _{k \in I} y_{k} L_{l}\left(F, x^{i}, x^{k}\right) \rightarrow \max _{k \in I} y_{k}+L_{-}\left(F, x^{i}, x^{k}\right) \text {. }
$$

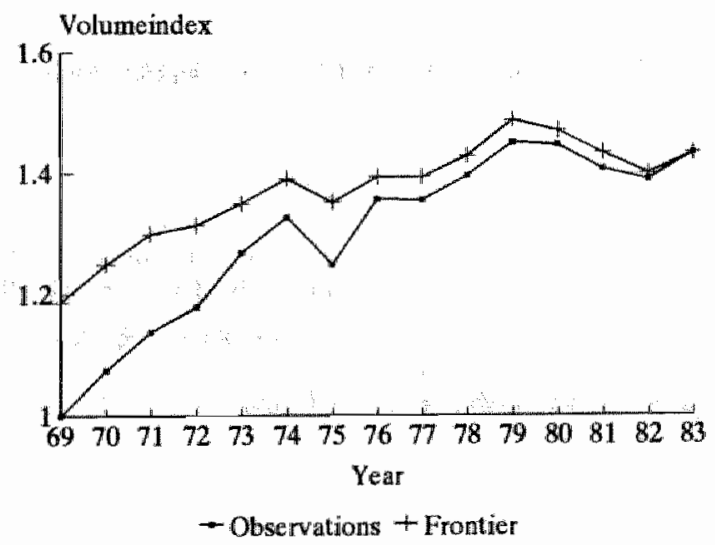

Figure 13.2 Minimal Frontier Production Funetion

Thus the above theorem prowides the minimal values for the frontier production function outputs. The hypothesis of profit maximization without 
observed output has to be satisfied in order that such values do exist. To satisfy this hypothesis may need an efficiency transformation. The previous section gives for the Dutch industry data an efficiency upper bound of $e=99.2 \%$. The minimal frontier production values for the transformed data, using this economical efficiency level, are given in Figure 13.2 .

We can conisider the ratio of the observed output and the minimal frontier production value as a technical efficiency coefficient. These ratios are given in Figure 13.3, which includes a logarithmic regression line fit. This figure gives insight in the existence of technical progress. It appears from the given graph that technical progress is presient in the Dutch industry. data. The logarithmic regression line fit resulted in a technical progress of 1.1\% per year $\left(R^{2}=0.87\right)$. In the following section the subject of technical progress is further elaborated.

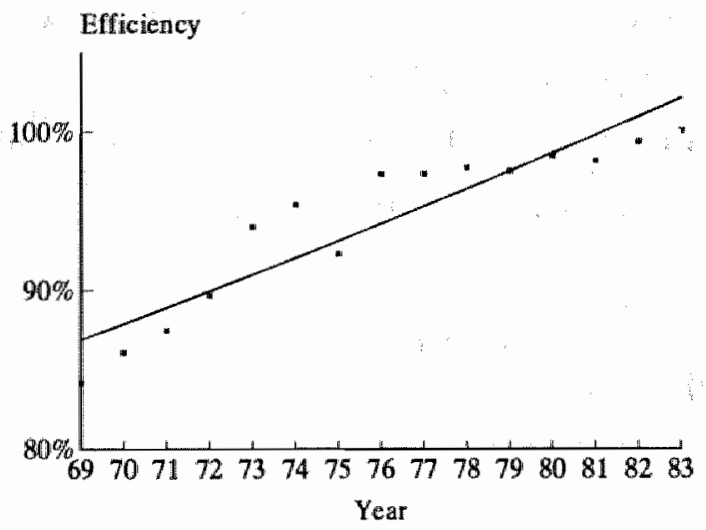

Figure 13.3 Technical Efficiency Upper Bound

\subsection{Technical Progress}

With the following theorem we may test whether there exists an increasing family of profit maximizing production functions for a given data set.

Theorem 13.8: Suppose $D=\left\{\left(c_{i}, x^{i}, y_{i}\right)\right\}_{i \in I}$ is a data set where $c_{i}: X \rightarrow \mathbb{R}_{+}$. Then the following conditions are equivalent:

(i) There exists a series production functions $f_{i}$, iel, such that $f_{j} \leq f_{i}$ if $j \leq i$, and $\left(c_{i}, x^{i}, y_{i}\right) \in \mathcal{D}_{p}^{o}\left(f_{i}\right)$ for all $i \in l$.

(ii) One has

$$
y_{j} \leq y_{i}+c_{i}\left(x^{j}\right)-c_{i}\left(x^{i}\right) \quad(i, j \in I: j \leq i)
$$

Proof: As the proof of Theorem 9.5 .

I applied condition (ii) of the above theorem to the Dutch industry data. As a result I obtained that only two observations have to be removed in order to achieve consistency with technically progressing profit maximization. The economical efficiency upper bound for the wholle data set is equal to $e=97.1 \%$, where the efficiency transformation of Example 2.3 is used. 
In the previous section I considered frontiev production functions: When we consider techmical progress a similar approach is possible. The following theorem is concerned with the mimimal possible output values of a frontier production function, assuming that technical progress is allowed.

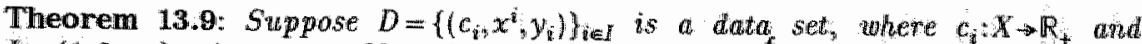
$I=\{1,2, \ldots\}$ is countable Let each family $F_{i}^{f}$ contan the technical progressing profut maximizing fronter production functions for pertod : Thus functions $f_{i}$ such that $f_{j} \leq f_{i}$ if $j \leq i$ and $\left(c_{i}, x^{i}, y_{i}\right) \in D_{p}\left(f_{i}\right)$ for all $i \in I$, satisfy $f_{i} \in F_{i}^{f}$ for every $i \in I$. Put $u_{1}=y_{1}$ and

$$
u_{i}=\max \left\{u_{j}+c_{i}\left(x^{i}\right)-c_{i}\left(x^{j}\right) \mid i, j \in I: j<i\right\} \cup\left\{y_{i}\right\} \quad(i=2, \ldots) .
$$

Then $L\left(F_{i}^{f}, x^{i}\right)=u_{i}$ for $a l l i \in I$.

Proof: Using Theorem 13.8 one can show that the given numbers $u_{i}$ are suitable as outputs $f_{i}\left(x^{i}\right)$ of a series technically progressing frontier production functions $f_{i} \in F_{i}, i \in I$. Furthermore, using the definition of $D_{p}^{f}$ one can show that these numbers are minimal. See also Theorem 9.7.

Application of the above idea of technically progressing frontier production functions to the Dutch industry data leads to the following results, The minimal value of the frontier function was equal to the observed output, i.e. $L\left(F_{i}^{f}, x^{i}\right)=u_{i}=y_{i}$, except for two years. These two technical inefficient years are 1975 and 1979 with technical efficiency coefficients $y_{i} / u_{i i}$ equal to $97.3 \%$ and $99.7 \%$ respectively.

\subsection{Hicks Neutral Change}

The theory concerning Hicks neutrall change is not completely analogous to linearly homogeneous producer demand. One has $\ln (t f(x))=\ln t+\ln f(x)$, thus for a complete analogy one should add a factor instead of multiplying the output by a factor. So, as appears from the following theorem, neutrat changing profit maximization cannot directly be derived from the results concerning neutral changing linearly homogeneous producer demand.

Theorem 13.9: Let $c: X \rightarrow \mathbb{R}_{+}$and $f: X \rightarrow \mathbb{R}$. Then

$$
\left(c, x^{\prime}, y\right) \in \mathcal{D}_{p}^{o}(t f) \Leftrightarrow\left(t^{-1} c, x^{n}, t^{-1} y\right) \in \mathcal{D}_{p}^{o}(f) \quad(t>0) .
$$

Proof: One has the equality

$$
\sup _{x \in X} t(x)-c(x)=t\left[\sup _{x \in X} f(x)-t^{-1} c(x)\right] \quad(t>0) .
$$

In contrast to the above we have for linearly homogeneous producer demand the equivalence

$$
\left(c, x^{u}, y\right) \in D_{d}^{o}(t f)=\left(c, x^{\prime}, t^{-1} y\right) \in D_{d}^{o}(f) \quad(t>0)
$$

where it is not necessary to divide the cost function by the factor $t$

For Hicks neutral changing linearly homogeneous producer demand, we can choose the values $t^{-1} y$ by choosing a suitable multiplication factor $t$. For profit maximization, however, this means also a change of the cost functions $t, c$. Therefore the following theorem, concerning Hicks neutral changing profit maximization, is not analogous to the corresponding theorem concerning linearly homogeneous producer demand.

Theorem 13.10: Suppose $D=\left\{\left(c_{i}, x^{i}, y_{i}\right)\right\}_{\text {ief }}$ is a data set, where $y_{i}>0$ and $c_{i}: X \rightarrow \mathbb{R}_{+}$for all $i \in I$. Then the following conditions are equiwalent:

(i) There exist a function $f: X \rightarrow \mathbb{R}$ and numbers $t_{i}>0$, such that

$$
\left(c_{i}, x^{i}, y_{i}\right) \in \mathcal{D}_{p}^{o}\left(t_{i} f\right) \quad(i \in I)
$$


(1) There expt walues $\phi_{i}=$ In $t_{1}$ such that

$$
\phi_{i}-\phi_{j} \leq a_{i j} \quad(i, j \in I)
$$

where

$$
a_{i j}=\ln \left(\left[y+c_{i}(x)-c_{i}\left(x^{3}\right)\right] / y\right) \quad(i, j \in I)
$$

Proof Condition (i) by Theorem 13.9 equivalent to the existence of a function $f$ and $t_{i}>0$, such that

$$
\left(t_{i}^{-1} c_{i}, x^{i}, t_{i}^{1} y_{i}\right) \in \mathcal{D}_{p}^{o}(f) \quad(i \in \mathbb{1})
$$

The latter is by Theorem 13.1 equivalent to the condition

$$
t_{j}^{-1} y_{j} \leq t_{i}^{-1}\left[y_{i}+c_{i}\left(x^{j}\right)-c_{i}\left(x^{i}\right)\right] \quad(i, j \in l)_{4}
$$

Which is equivalent to (ii).

1 used condition (ii) in the above theorem to test the hypothesis of Hicks neutral changing profit maximization for the Dutch industry data This condition can be tested using the theory given in Appendix $C$. Five observations have to be removed if we assume economical efficient behaviour. For the whole data set I derived an efficiency upper bound of $e=99.8 \%$, using the efficiency transformation of Example 2.3 .

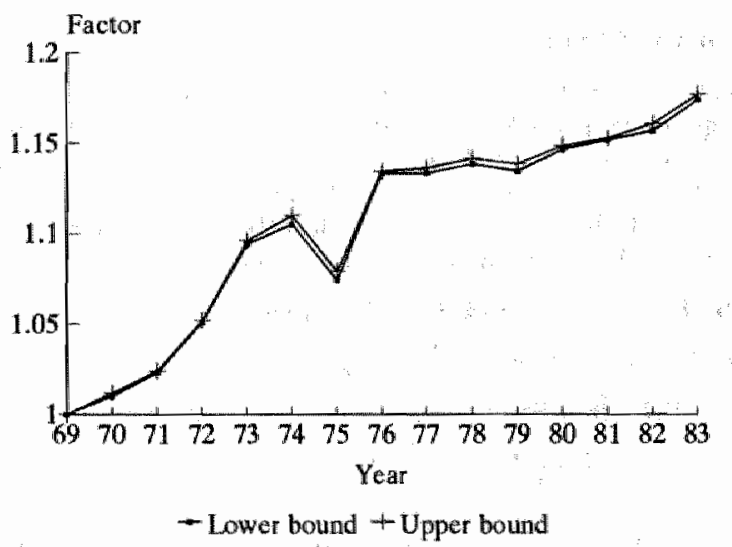

\section{Figure 13.4 Bounds Hicks Neutral Change}

For the transformed data, belonging to this efficiency upper bound, I computed the lower and upper bounds for the factor of Hicks neutral change, relative to the first observation. "These bounds are in terms of Theorem 13.10 equal to

$$
-a_{1 i}^{*} \leq t_{i} / t_{1} \leq e^{a_{i 1}^{*}} \quad(i \in I)
$$

The resulting bounds, shown in Figure 13.4, are very close together.

The following theorem concening Hicks neutral progress is analogous to Theorem 13.10. There is only one difference: we assume that the multiplying factors $t_{\mathrm{i}}$ increase for later observation periods $t_{\text {. }}$ 
Theorem 13.11: Suppose $D=\left\{\left(c_{i}, x^{i}, y_{i}\right)\right\}_{\mathrm{i} \in \mathrm{I}}$ is a data set where $y_{\mathrm{i}}>0$ and $c_{\|} X \rightarrow \mathbb{R}_{*}$ for all $i \in I$. Then the following conditions are equivilent:

(1) There exist a function $f: X \rightarrow \mathbb{R}$ and numbers $t_{i}>0$, such that $t_{j} \leq t_{i}$ if $j \leq t_{4}$ and

$$
\left(c_{i}, x^{i}, y_{i}\right) \in \mathcal{D}_{p}^{0}\left(t_{i} f\right) \quad(i \in I) .
$$

(ii) There exist walues $\phi_{i}=\ln t_{i}$ such that

$$
\phi_{i}-\phi_{j} \leq \min \left\{a_{i j}, b_{i j}\right\}:(i, j \in I)
$$

where

$$
\begin{aligned}
& a_{i j}=\ln \left(\left[y_{i}+c_{i}\left(x^{j}\right)-c_{i}\left(x^{i}\right)\right] / y_{j}\right) \quad(i, j \in I), \\
& b_{i j}= \begin{cases}0 & (i, j \in I: i \leq j), \\
\infty & \text { (otherwise). }\end{cases}
\end{aligned}
$$

Proof: We may apply Theorem 13.10 , concerned with Hicks neutral change, by adding the condition that the numbers $t_{i}$ are increasing. In that case we obtain from the conditions in Theorem 13.10, the conditions (i) and (ii) in this theorem.

When we assume that there is Hicks neutral progress for the Dutch industry data, the same number of observations have to be removed as for Hicks neutral change. The economical efficiency upper bound, however, is a bit lower than obtained for Hicks neutral change. The computed bound is $e=97.1 \%$.

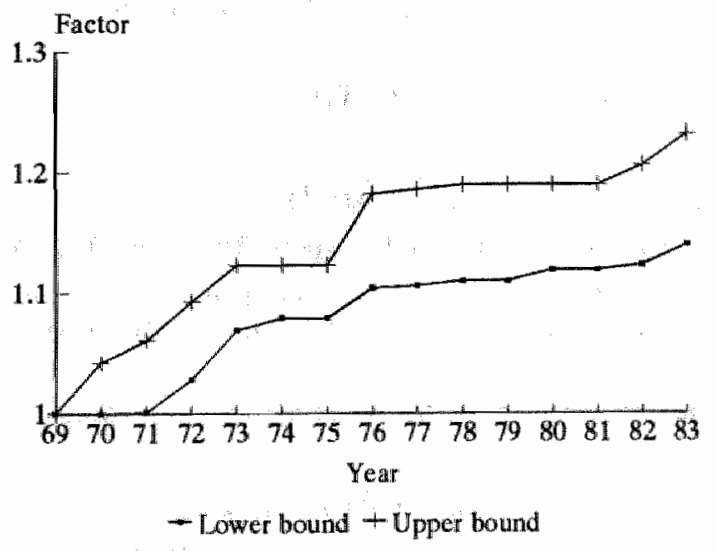

Figure 13.5 Bounds Hicks Neutral Progress

Figure 13.5 displays the lower and upper bound of the Hicks neutral progress factor for the transformed data, using this economical efficiency level. The factor for Hicks netutral progress is computed relative to the first observation. The bounds are now more diverging than in the case of Hicks neutral change, because the factor of Hicks neutral progress may not decrease. 


\subsection{Neutral Change}

Meutral change concerning both profit maximization and linearly homogeneous: producer demand is completely analogous as is shown by the following theorem.

Theorem 13.12: Suppose $c: X_{1} \times X_{2} \rightarrow \mathbb{R}_{4}, f: X_{1} \times X_{2} \rightarrow \mathbb{R}_{3}$ where $X_{2}$ is a cone. Then

$$
\left(c, x^{\prime}, y\right) \in \mathcal{D}_{p}^{o}\left(f\left(x_{1}, t x_{2}\right)\right) \Leftrightarrow\left(c\left(x_{1}, t^{-1} x_{2}\right),\left[x_{1}^{*}, t x_{2}^{*}\right], y\right) \in D_{p}^{o}(f) \quad(t>0) .
$$

Proof: Suppose $\left(c, x^{\prime}\right) \in \mathcal{D}_{p}\left(f\left(x_{1}, t x_{2}\right)\right)$. This is by definition of profit maximization equivallent to

$$
f\left(x_{1}, t x_{2}\right)-c\left(x_{1}, x_{2}\right) \leq f\left(x_{1}^{\prime}, t x_{2}^{\prime}\right)-c\left(x_{1}^{\prime}, x_{2}^{\prime \prime}\right) \quad\left(x_{1} \in X_{1}, x_{2} \in X_{2}\right) .
$$

By putting $x_{2}^{\prime \prime}=t x_{2}$, we obtain that this is equivalent to

$$
f\left(x_{1}, x_{2}^{\prime n}\right)-c\left(x_{1}, t^{-1} x_{2}^{\prime \prime}\right) \leq f\left(x_{1}^{\prime}, t x_{2}^{\prime}\right)-c\left(x_{1}^{\prime}, t^{-1} t x_{2}^{\prime}\right) \quad\left(x_{1} \in X_{1}, x_{2}^{\prime \prime} \in X_{2}\right)
$$

which is by the definition of profit maximization equivalent to

$$
\left(c\left(x_{1}, t^{-1} x_{2}\right),\left[x_{1}, t x_{2}\right], y\right) \in \mathcal{D}_{p}(f) \text {. }
$$

Obviously, the above result concerning $\mathcal{D}_{p}$ without observed output is also valid for $\mathcal{D}_{p}^{\circ}$ with observed output.

The following theorem states necessary and sufficient conditions for the existence of a neutral changing profit maximizing production function. The theorem is an analogy of Theorem 9.11, which considers a similar case concerning linearly homogeneous producer demand.

Theorem 13.13: Suppose $D=\left\{\left(c_{i}, x^{i}, y_{i}\right)\right\}_{\text {ier }}$ is a data set, where $c_{i}: X_{1} \times X_{2} \rightarrow \mathbb{R}_{+}$and $X_{2}$ is a cone. Let $t_{i}>0$ for all $i \in I$. Then the following conditions are equivalent:

(i) There exists a function $f: X_{1} \times X_{2} \rightarrow \mathbb{R}$ such that $\left(c_{i,}, x^{i}, y_{i}\right) \in D_{p}^{o}\left(f_{i}\right)$ for all $i \in I$, where

$$
f_{i}(x)=f\left(x_{1}, t_{i} x_{2}\right) \quad\left(i \in I, x \in X_{1} \times X_{2}\right) .
$$

(ii) One has

$$
y_{j} \leq y_{i}+c_{i}\left(x_{1}^{j}, t_{i}^{-1} t_{j} x_{2}^{j}\right)-c_{i}\left(x^{i}\right) \quad\left(i_{i} j \in I\right) \text {. }
$$

Proof: As the proof of Theorem 9.11, where Theorem 13.12 may be used.

For a data set of prices and quantities, one may use the following theorem to check the existence of a neutral changing profit maximizing production function.

Theorem 13.14: Suppose $D=\left\{\left(c_{i}, x^{i}, y_{i}\right)\right\}_{\text {iel }}$ is a finite data set and all functions $c_{i}: X_{1} \times X_{2} \rightarrow \mathbb{R}_{+}$are of the form

$$
c_{i}(x)=v_{i}\left(x_{1}\right)+w_{i}\left(x_{2}\right) \quad\left(x \in X_{1} \times X_{2}\right),
$$

where $w_{y_{i}}: X_{2} \rightarrow \mathrm{R}_{*}$ is linearly homogeneous and $X_{2}$ is a cone. Then the following condations are equivalent:

(i) There exist a function $f: X_{1} \times X_{2} \rightarrow \mathbb{R}$ and $t_{i}>0$ such that for the functions

$$
f_{i}(x)=f\left(x_{1}, t_{i} x_{2}\right) \quad\left(i \in I, x \in X_{1} \times X_{2}\right)
$$

one has $\left(c_{i}, x^{i}, y_{i}\right) \in \mathcal{D}_{p}^{o}\left(f_{i}\right)$.

(ii) There exist values $\phi_{i}=\ln t_{i}$ such that

$$
\phi_{i}-\phi_{j} \leq a_{i j} \quad(i, j \in I),
$$

where 


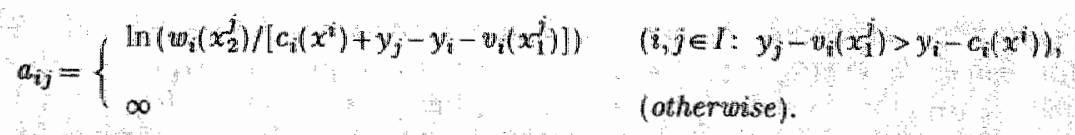

Proof: Condition (i) is by Theorem 13.13 equivalent to the existence of numbers $t_{i}>0$ such that

$$
y_{j} \leq y_{i}+c_{i}\left(x_{1,}^{j}, t_{i}^{11} t_{j} x_{2}^{j}\right)-c_{i}\left(x^{i}\right) \quad(i, j \in I)
$$

Since we have by assumption

$$
c_{i}\left(x_{1}^{j}, t_{i}^{-1} t_{j} x_{2}^{j}\right)=v_{i}\left(x_{1}^{j}\right)+t_{i}^{-\mathbb{1}} t_{j} u_{i}\left(x_{2}^{j}\right) \quad(i, j \in I),
$$

these inequalities can be written as

$$
\left(t_{i} / t_{j}\right)\left[c_{i}\left(x^{i}\right)+y_{j}-y_{i}+v_{i}\left(x_{1}^{j}\right)\right] \leq w_{i}\left(x_{2}^{j}\right) \quad(i, j \in I),
$$

which is equivalent to the given inequalities in condition (ii).

I investigated the implications of Theorem 13.14 for both labour and capital in the Dutch industry data. One might say that the hypothesis of neutra]. changing capital is more satisfactory than the hypothesis of neutral changing labour. The hypothesis of neutral changing labour can be satisfied by removing six observations, while for neutral changing capital only two observations have to be removed. For the whole data set the economical efficiency upper bound concerning the assumption of neutral changing labour and capital is $99.3 \%$ and $99.8 \%$ respectively, using the efficiency transformation of Example 2.3.

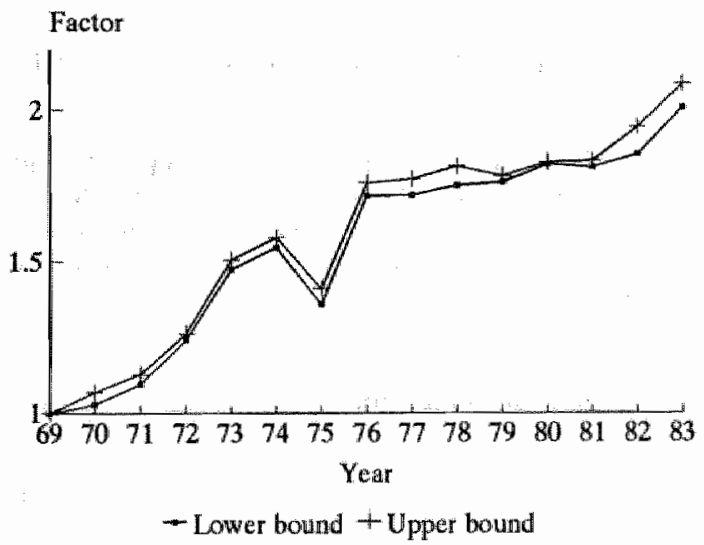

Figure 13.6 Bounds Neutral Change Labour

The weak closure of the matrix $A$ in the above theorem provides lower and upper bounds for the factor of neutral change. These bounds, relative to the factor corresponding to the first observation, are

$$
\mathrm{e}^{-a_{1, i}^{*}} \leq t_{i} / t_{1} \leq \mathrm{e}^{a_{i 1}^{*}} \quad(i \in I) \text {. }
$$

Figure 13.6 and 13.7 display the bounds on the factor of neutral change for the transformed data, where the efficiency level for the tramsformation corresponds to the economical efficiency upper bound level. The resultis in 
Pgure 13.7 concerning capital are quite different compared to the results for labour in Figure 13.6 . The lower bound for capital is strongly increasing and, while the bounds for labour are tight, the bounds for capital are diverging. After 1972 there is no upper bound information available for the neutral charge of capital: the upper bound for these observations is equal to infinity. This phenomenon is also observed in Chapter 9, which is concerned with linearly homogeneous producer demand.

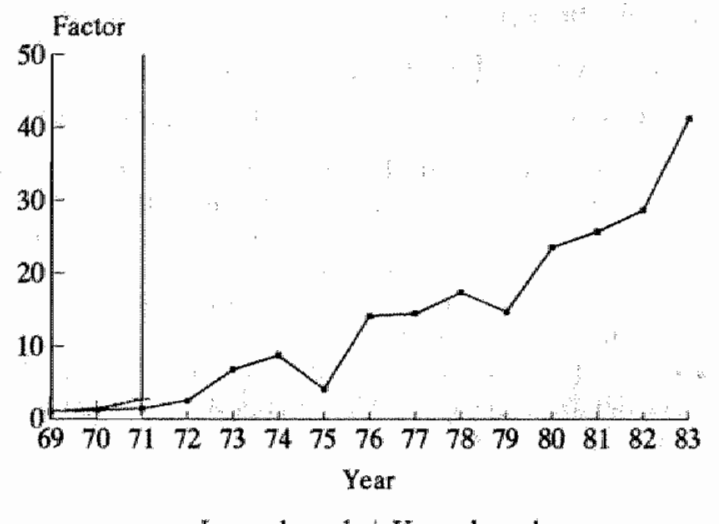

- Lower bound - Upper bound

Figure 13.7 Bounds Neutral Change Capital

How can we explain this phenomenor of infinite upper bounds? The arguments are similar to the arguments, given in Chapter 9, that apply to linearly homogeneous demand. Suppose the data are consistent with technically progressing profit maximization of economical efficiency level $e$. Then wre have in Theorem 13.8 the relation

$$
y_{j} \leq y_{i}+c_{i}\left(x^{j}\right)-c_{i}\left(x^{i}\right) \quad(i, j \in I: j \leq i) .
$$

This makes it likely that we have moreover

$$
y_{j} \leq y_{i}+c_{i}\left(x^{j}\right)-c_{i}\left(x^{i}\right)-w_{i}\left(x_{2}^{j}\right)=y_{i}+v_{i}\left(x_{1}^{j}\right)-c_{i}\left(x^{i}\right) \quad(i, j \in I: j<i),
$$

whicht is equivalent to $a_{i j}=\infty$ for $j<i$. This will be especially likely when the terms $\psi_{i}\left(x_{2}^{3}\right)$ are relatively small. This is the case for capital and not for labour: capital and labour costs are approximately $3 \%$ and $25 \%$ respectively, of the total costs $c_{i}\left(x^{i}\right)$. Now, suppose we have $a_{i j}=\infty$ for $j<i$. Then every path from $j$ to $i$ with $i>j$ does always encounters an infinite value $a_{k t}=\infty$, with $k<l$. This implies that we have also $a_{i j}^{*}=\infty$ for $j<i$. This in turn means that we find infinite upper bounds

$$
t_{i} / t_{1}<e^{a_{i 1}^{*}}=\infty \quad(i>1)
$$

In summary: when the data are approximately consistent with technically progressing profit maximization and the neutral changing costs $w_{i}\left(x_{2}^{j}\right)$ are relatively small, we may expect the occurrence of infinite upper bounds on $t_{i} / t_{1}$. We observe this phenomenon in Figure 13.7 for neutral changing capital. It does not appear in Figure 13.6 concerning neutral changing 
labour, because labour costs are substantially higher than capital costs.

When we assume that there is neutral progress, instead of neutral change, we have to include the additional restriction that the scale factors are increasing. The resulting restrictions are given in the following theorem.

Theorem 13.15: Concerning Theorem 13.14, the following conditions are equivalent:

(i) There exst $t_{i}>0$ satisfying Theorem 13.14(i), such that $t_{j} \leq t_{g}$ if $j \leq i$.

(ii) There exist values $\phi_{i}=\ln t_{i}$ such that

$$
\phi_{i}-\phi_{j} \leq \min \left\{a_{i j}, b_{i j}\right\} \quad(i, j \in I)
$$

where $a_{i j}$ is given in Theorem 13.14 and.

$$
b_{i j}= \begin{cases}0 & (i, j \in I: i \leq j), \\ \infty & \text { (otherwise) }\end{cases}
$$

Proof: As the proof of Theorem 13.14. We only add to Theorem 13.14 (i) the condition that we have

$$
t_{j} \leq t_{i} \quad(i, j \in I: j \leq i)_{n}
$$

which means that we must add to Theorem 13.14 (ii) the additional condition

$$
\phi_{i}-\phi_{j} \leq 0 \quad(i, j \in I: i \leq j) \text {. }
$$

In order to accept the assumption of neutral change we have to remove seven and three observations for labour and capital respectively. The assumption of neutral progress is stronger than the assumption of neutral change, thus we obtain a larger number of removed observations than in the case of neutral change. Also because of the stronger assumption, we obtain a lower economical efficiency upper bound. This economical efficiency upper bound concerning the Dutch industry data was in both cases, for labour and capital, equal to $97.1 \%$. Figure 13.8 and 13.9 display the lower and upper bounds on $t_{i} / t_{1}$ that correspond to the transformed data for this efficiency upper bound.

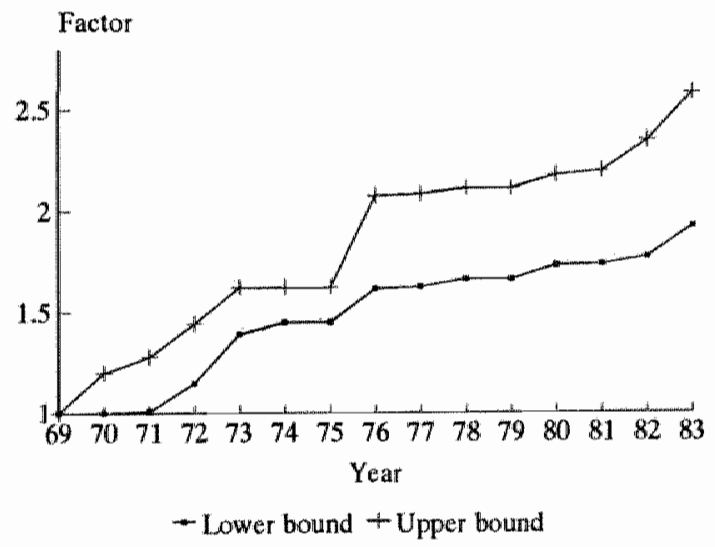

Figure 13.8 Bounds Neutral Progress Labour 
128 Profut Maximization

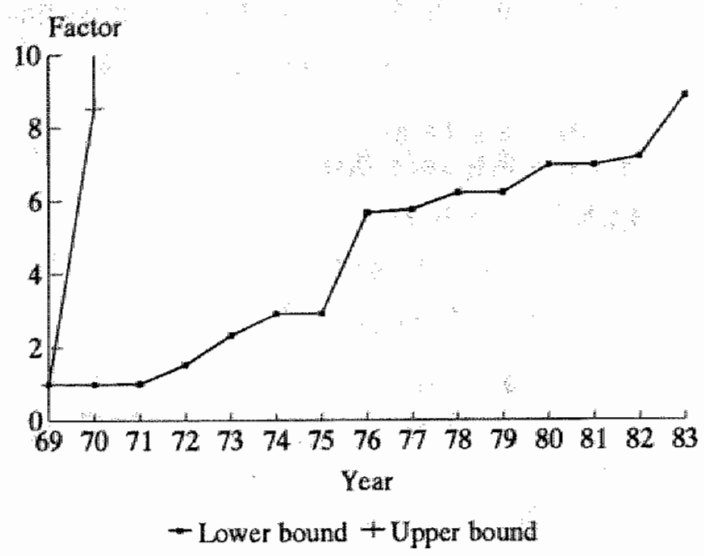

Figure 13.9 Bounds Neutral Progress Capital 


\section{HOMOTHETIC PROFIT MAXIMIZATION}

\subsection{Introduction}

In this chapter we shall consider the existence of homothetic production functions for producer data with observed output. It will be shown that profit maximization for a homothetic production function can be considered as a two stage process. A homothetic function is a monotonic transformation of a linearly homogeneous function. The two stages for such a function are as lollows. First, the linearly homogeneous function has to satisfy costi minimization. Secondly, the monotonic transformation acts as a simplified profit maximizing production function.

\subsection{Existence of a Homothetic Production Function}

Suppose mof $f(x)$ is a homothetic function where $m$ is strictly increasing and $f$ is linearly homogeneous. Now, consider the profit maximization problem

$$
\sup _{x} \operatorname{mof}(x)-c(x) \text {. }
$$

The idea is to transform this problem to a problem of a symmetric form, using the same approach as is earlier used for weak separability. Let the transformation $T_{f}$ be defined by

$$
\left(T_{f} c\right)(x)=\inf _{f(v)=f(x)} c(v) \quad(x \in X) .
$$

Now we have

$$
\sup _{x} m \circ f(x)-c(x)=\sup _{x} \sup _{f(v)=f(x)} m \circ f(v)-c(v)=\sup _{x} m_{\circ} f(x)-\left(T_{f} c\right)(x) .
$$

Because $\left(T_{f} c\right)(x)$ can be written in the form $d(f(x))$, any optimal solution $x^{\prime}$ has to satisfy the requirement that $z=f\left(x^{\prime}\right)$ solves the simplified profit maximization problem

$$
\sup m(z)-d(z) \text {. }
$$

Furthermore, because any optimal solution $x^{\prime}$ is a cost minimizing solution, another condition that has to be satisfied is $\left(c, x^{4}\right) \in \mathcal{D}_{c}(f)$. Using this approach, the proof of the existence of a profit maximizing homothetic production function is relatively easy.

Theorem 14.1: Suppose $D=\left\{\left(c_{i}, x^{i}, y_{i}\right)\right\}_{\text {ie }}$ is a finite data set, where $c_{i}: X \rightarrow \mathbb{R}_{+}$ are linearly homogeneors and $c_{i}\left(x^{i}\right)>0$, for all $i \in I$, and $X$ is a cone. Then the folloning conditions are equivalent:

(i) There exists a homothetic function $h \in \mathcal{F}_{p}^{o}(D)$.

(ii) There exists a linearly homogeneous function $f: X \rightarrow \mathbb{R}$ and an indreasing function $m: \mathbb{R} \rightarrow \mathbb{R}$ such that $f \in \mathcal{F}_{d}\left(D^{\dagger}\right)$ and $m \in \mathcal{F}_{p}^{o}\left(\left\{\left(P_{i}, f_{i}, y_{i}\right)\right\}_{\text {ieI }}\right)$, where

$$
f_{i}=f\left(x^{i}\right), P_{i}=c_{i}\left(x^{i}\right) / f\left(x^{i}\right) \quad(i \in I) .
$$

(iii) There exist values $\phi_{i}$ such that

$$
\phi_{i}-\phi_{j} \leq \min \left\{a_{i j}, b_{i j}\right\} \quad(i, j \in I)_{4}
$$

where

$$
a_{i j}=\ln \left(c_{j}\left(x^{i}\right) / c_{j}\left(x^{j}\right)\right) \quad(i, j \in I)
$$




$$
b_{i j}= \begin{cases}\ln \left(c_{i}\left(x^{i}\right) /\left[y_{j}-y_{i}+c_{i}\left(x^{i}\right)\right]\right) & \left(i_{j} j: y_{j}-y_{i}+c_{i}\left(x^{i}\right)>0\right), \\ \infty & \text { (otherwise) }\end{cases}
$$

Proof $(i) \Rightarrow$ (ii): Suppose $\hbar=$ mof $\in \mathcal{F}_{p}^{o}(D)$ is a homothetic function. Then one has $h \in \mathcal{F} d(D)$ by Theorem 12.1, and hence $f \in \mathcal{F}_{c}(D)$. Furthermore, since $h \in \mathcal{F}_{P}^{0}(D)$ by assumption, ore has

$$
m_{o} f\left(\lambda x^{i}\right)-c_{i}\left(\lambda x^{i}\right) \leq m o f\left(x^{i}\right)-c_{i}\left(x^{i}\right) \quad(\lambda \geq 0, t \in I)
$$

thus for $\lambda=\eta f\left(x^{i}\right)^{-1}$ and $y_{i}=n o f\left(x^{2}\right)$ we obtain

$$
m(\eta)-P_{i} \eta \leq y_{i}-P_{i} f_{i}
$$

so that $n \in \mathcal{F}_{p}\left(\left\{\left(P_{i}, f_{i}, y_{i}\right)\right\}_{i \in t}\right)$.

(ii) $\Rightarrow$ (iii): Suppose that $m$ and $f$ satisfy condition (ii). Since $f \in \mathcal{F}_{c}(D)$ is linearly homogeneous, we may apply Theorem 3.4 to obtain $f\left(x^{i}\right)>0$ for all $i \in I$, and by Theorem 9.1 we have then

$$
f_{i} / f_{j} \leq c_{j}\left(x^{i}\right) / c_{j}\left(x^{j}\right) \quad(i, j \in I)
$$

Furthermore, $m \in \mathcal{F}_{p}\left(\left\{\left(P_{i} f_{i}, y_{i}\right)\right\}_{\text {iel }}\right)$ implies

$$
y_{j}-c_{i}\left(x^{i}\right) f_{i}^{-1} f_{j}=y_{j}-P_{i} f_{j} \leq y_{i}-P_{i} f_{i}=y_{i}-c_{i}\left(x^{i}\right) \quad(i, j \in I) .
$$

Thus

$$
\ln f_{i}-\ln f_{j} \leq \min \left\{a_{i j}, b_{i j}\right\} \quad(i, j \in I)
$$

and (iii) follows.

(iii) $\Rightarrow$ (i): Suppose there exist $f_{8}>0$ such that

$$
\ln f_{i}-\ln f_{j} \leq \min \left\{a_{i j}, b_{i j}\right\} \quad(i, j \in I) .
$$

Now, let

$$
P_{i}=c_{i}\left(x^{i}\right) / f_{i} \quad(i \in I),
$$

and put

$$
\begin{array}{ll}
f(x)=\min _{i \in I} f_{i} c_{i}(x) / c_{i}\left(x^{i}\right) & (i \in I, x \in X), \\
m(\eta)=\min _{i \in I} y_{i}+P_{i}\left(\eta-f_{i}\right) & (i \in I, \eta \in \mathbb{R}) .
\end{array}
$$

From the definition of $A$ and $B$ we can derive $f\left(x^{i}\right)=f_{i}$ and $m\left(f_{i}\right)=y_{i}$, for all $i \in 1$. Thus the choice of $m$ and $f$ implies

$$
\begin{aligned}
m(f(x))-c_{i}(x) & \leq y_{i}+P_{i}\left[f(x)-f_{i}-c_{i}(x)\right. \\
& \leq y_{i}+P_{i}\left[f_{i} c_{i}(x) c_{i}\left(x^{i}\right)-f_{i}\right]-c_{i}(x) \\
& =y_{i}-c_{i}\left(x^{i}\right) \quad\left(i \in I_{i}, x \in X\right) .
\end{aligned}
$$

Hence mof $\in \mathcal{F}_{p}^{\circ}(D)$, which is clearly a homothetic function with the desired propertiles.

Remark: We may extend Theorem 14.1 to the case where the data set $D$ contains an infinite number of elements. However, in case of infinite data sets it may happen that the constructed function $m$ in the proof of (iii) (i) is not a strictly increasing - but an increasing - function. For infinite data sets we can only prove the equivalence of the conditions (i), (ii) and (iii), when we drop in the definition of a nomothetic function the condition that $m$ is strictly increasing. Such a theorem concerning increasing functions $m$ - is valid, because the entire proof of Theorem $\mathbb{1 4 . 1}$, only refers to the assumption that $m$ is increasing.

The results in the above theorem are related to the use of price indices. The values $P_{i}$ are in fact price indices corresponding to the volumes $f_{i}$ of the 
linearly homogeneous function. The theorem makes the following two stage process clear concerning profit maximization with a homothetic function mof: there is cost minimization on a lower level for the linearly homogeneous function $f \in \mathcal{F}(D)$ and there is profit maximization on a higher level for the monotonic transformation $m \in \mathcal{F}_{p}^{o}\left(\left\{\left(P_{i}, f_{i}, y_{s}\right)\right\}_{i \in i}\right)$.

In Varian (1984, Theorem 8) one may find weaker necessary and sufficient conditions concerning homothetic profit maximization. However, these conditions are only necessary conditions. The sufficiency proof in Varian (1984) contains a small error. Varian did forget to show that his constructed production function satisfies $m\left(f\left(x^{i}\right)\right)=y_{i}$ or - in the notation used by Varian - $f\left(\Phi\left(x^{i}\right)\right)=y^{i}$.

Application of condition (iii) of Theorem 14.1 to the Dutch industry data shows a bad fit with homothetic profit maximization. To achieve consistency with homothetic profit maximization it appears that all observations, except one, have to be removed. I could, however, save myself the trouble to compute these results. Chapter 13 contains the same results concerning profit maximization without restrictions on the production function.

We may derive restrictions on the underlying linearly homogeneous function in the same way as is derived for homothetic cost minimization in Chapter 10 . These restrictions are given in the following theorem.

Theorem 14.2: Suppose $D=\left\{\left(c_{i}, x^{i}, y_{i}\right)\right\}_{\text {ieI }}$ is a finite data set, where $c_{i}: X \rightarrow \mathbb{R}_{+}$ are linearly homogeneous and $c_{i}\left(x^{i}\right)>0$, for all $i \in I$, and $X$ is a cone. $P$ ut

$$
\begin{aligned}
& F=\{f: X \rightarrow \mathbb{R} \mid f \text { linearly homogeneous, } \\
& \text { there is a monotomic function m such that mof } \left.\in \mathcal{T}_{p}^{o}(D)\right\}, \\
& d_{i j}=\min \left\{a_{i j}, b_{i j}\right\} \quad(i, j \in I),
\end{aligned}
$$

with $a_{i j}$ and $b_{i j}$ as given in Theorem 14.1.

If $F \neq \emptyset$ then $U_{\gamma}\left(F, ; x^{j}\right) \in F$ for all $j \in I$ and

$$
\begin{aligned}
& U_{/}\left(F, x, x^{j}\right)=\inf _{i \in I}\left[c_{i}(x) / c_{i}\left(x^{i}\right)\right] \mathrm{e}^{d_{i j}^{*}} \quad\left(j \in I_{\eta}, x \in X\right), \\
& U_{/}\left(F, x^{i}, x^{j}\right)=\mathrm{e}^{d_{i j}^{*}}, U_{/}\left(F, x^{i}, x^{j}\right)^{-\|}=L_{/}\left(F, x^{j}, x^{j}\right) \quad(i, j \in I) .
\end{aligned}
$$

Proof: An outline of the proof is as follows. Put

$$
f_{j}(x)=\inf _{i \in I}\left[c_{i}(x) / c_{i}\left(x^{i}\right)\right] \mathrm{e}^{d_{i j}^{*}} \quad(j \in I, x \in X) .
$$

Then we can show that we have $f_{j} \in F^{*}$ and thus $U_{j}\left(F, x, x^{j}\right) \geq f_{j}(x)$. Furthermore, in a. similar way as in the proof of Theorem 3.12, we can show that $U_{/}\left(F, x, x^{j}\right) \leq f_{j}(x)$. This means that we have $U_{\lambda}\left(F, x, x^{j}\right)=f_{j}(x)$. The remaning part of the proof is also similar to the proof of Theorem 3.12 .

With the above theorem one may derive bounds on the monotonic function $m$. In Chapter 10 such bounds are given in Figure 10.1, which concerns the case of homothetic cost minimization. However, it was not possible to apply the above theorem to the Dutch industry data, because these data are not compatibie at all with homothetic profit maximization. This was already apparent in Chapter 10. As is shown in Figure 10.1, there exists no concdive cost minimizing homothetic function for the data. Because any profit maximizing function for price and quantity data has to be concave and cost minimizing, there exists thus no homothetic profit maximizing function for the Dutch industry data. To allow such a function to exist, we might try to include, for example, the assumption of Hicks neutral progress. 


\section{WEAKLY SEPARABLE PROFIT MAXIMIZATION}

\subsection{Introduction}

A. weakly separable function consists of a subfunction and an aggregator function. In the previous chapter a two stage optimizing process was used to attack the problem of profit maximization with a homothetic production function. A similar approach is also convenient in case of weakly separable production functions. In this chapter it will be shown that weakly separable profit maximization can be conceived as a cost minimization stage for the subfunction and a profit maximization stage on a higher level for the aggregator function.

\subsection{Existence of an Aggregator Function}

The basic idea in this chaptier is the observation that weakly separable functions $f(x)=g\left(x_{1}, h\left(x_{2}\right)\right)$ satisfy

$$
\sup _{x} f(x)-c(x)=\sup _{x} f(x)-\left(T_{h} c\right)(x)
$$

where the transformation $T_{h}$ is defined by

$$
\left(T_{h} c\right)\left(x_{1}, x_{2}\right)=\inf _{h(v)=h\left(x_{2}\right)} c\left(x_{1}, v\right) \quad\left(x \in X_{1} \times X_{2}\right)
$$

Moreover, in case $g\left(x_{1}, 7\right)$ is increasing in $\eta$, we may use the transformation

$$
\left(T_{h} c\right)\left(x_{1}, x_{2}\right)=\inf _{h(v) \geq h\left(x_{2}\right)} c\left(x_{1}, v\right) \quad\left(x \in X_{1} \times X_{2}\right) .
$$

Now, $\left(\boldsymbol{T}_{h} c\right)(x)$ can be written in the form $d\left(x_{1}, h\left(x_{2}\right)\right)$, hence the optimal $x_{1}^{\prime}$ and $\eta=h\left(x_{2}^{\prime}\right)$ are a solution of

$$
\sup _{x_{1}, \eta \eta} g\left(x_{1}, \eta\right)-d\left(x_{1}, \eta\right)
$$

Furthermore, because any optimal solution $x^{*}$ is a cost minimizing solution, the condition $\left(\boldsymbol{T}_{h} c\right)\left(x^{\prime}\right)=c\left(x^{\prime}\right)$ has to be satisfied.

So, we can transform a profit maximization problem with a weakly separable production function into a profit maximization problem of a symmetric form. This observation leads to conditions, which assure the existence of an aggregator function, given a data set on $X_{1} \times X_{2}$ and a (sub)function $h: X_{2} \rightarrow \mathbb{R}$.

Theorem 15.1: Suppose $D=\left\{\left(c_{1}, x^{i}, y_{j}\right)\right\}_{i \in l}$ is a finite data set, where $a_{i}: X_{1} \times X_{2} \rightarrow \mathbb{R}_{+}$. Suppose $h: X_{2} \rightarrow \mathbb{R}$ and let the transformation $T_{l_{2}}$ of any function $f: X_{1} \times X_{2} \rightarrow \mathbb{R}$ be defined by

$$
\left(T_{h} f\right)\left(x_{1}, x_{2}\right)=\inf _{h(v) \geq h\left(x_{2}\right)} f\left(x_{1}, v\right) \quad\left(x \in X_{1} \times X_{2}\right) .
$$

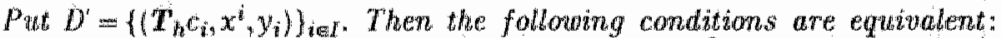

(1) There exists a weakly separable function $f \in \mathcal{F}_{p}^{o}(D)$ such that $T_{h} f=f$.

(ii) $\left(T_{h} c_{i}\right)\left(x^{i}\right)=c_{i}\left(x^{i}\right)$ for all $i \in I$, and $\mathcal{F}_{p}^{0}\left(D^{\prime}\right) \neq \varnothing$.

Proof $(i) \Rightarrow$ (ii): Suppose $f \in \mathcal{F}_{p}(D)$ is weakly separable such that $T_{h} f=f$. Then $f \in \mathcal{F}_{\mathrm{e}}(D)$ and by Theorem 4.5 one has $\left(T_{h} c_{i}\right)\left(x^{i}\right)=c_{i}\left(x^{i}\right)$ for all $i \in I$.

To complete the proof, I will show that $f \in \mathcal{F}_{p}^{o}\left(D^{\prime}\right)$. Let $i \in I$ and $x \in X_{1} \times X_{2}$. Because $T_{h} f=f \in \mathcal{F}_{p}^{o}(D)$, we have

$$
f\left(x_{1}, x_{2}\right)-c_{i}\left(x_{1}, v\right) \leq f\left(x_{1}, v\right)-c_{i}\left(x_{1}, v\right) \leq y_{i}-c_{i}\left(x^{i}\right)
$$


for any $v \in X_{2}$ such that $h(v) \geq h\left(x_{2}\right)$. Thus

$$
f(x)-\left(\boldsymbol{T}_{h} c_{i}\right)(x) \leq y_{i}-c_{i}\left(x^{i}\right)=f\left(x^{i}\right)-\left(\boldsymbol{T}_{h} c_{i}\right)\left(x^{i}\right) \quad(x \in X, \text { if } \in),
$$

so that $f \in \mathcal{F}_{p}^{0}\left(D^{\prime}\right)$

(iii) $\Rightarrow$ (i): Suppose $c_{i}\left(x^{i}\right)=\left(T_{h} c_{i}\right)\left(x^{i}\right)$, for all $i \in I$, and $\mathcal{F}_{p}^{0}\left(D^{*}\right) \neq \omega$. Put

$$
f(x)=\min _{i \in I} y_{i}+\left(\boldsymbol{T}_{h} c_{i}\right)(x)-\left(\boldsymbol{T}_{h} c_{i}\right)\left(x^{i}\right) \quad(x \in X) .
$$

Then one has $f \in \mathcal{F}_{p}^{\circ}\left(D^{\prime}\right)$ by Theorem 13.6 and moreover $T_{h} f=f$. Now, noting that one has $\boldsymbol{T}_{h} c_{i} \leq c_{i}$ by definition of $\boldsymbol{T}_{h}$ and $\left(\boldsymbol{T}_{h} c_{i}\right)\left(x^{i}\right)=c_{i}\left(x^{i}\right)$, for all $i \in l$, we obtain

$$
f(x)-c_{i}(x) \leq y_{i}+\left(T_{h} c_{i}\right)(x)-\left(T_{h} c_{i}\right)\left(x^{i}\right)-c_{i}(x) \leq y_{i}-c_{i}\left(x^{i}\right) \quad(x \in X, i \in I),
$$

which implies that we have $f \in \mathcal{F}_{p}^{o}(D)$.

Note that that we can also prove a similar theotem as the above, using the transformation

$$
\left(T_{h} f\right)\left(x_{1}, x_{2}\right)=\inf _{h(v)=h\left(x_{2}\right)} f\left(x_{1}, v\right)
$$

\subsection{Existence of a Weaklly Separable Function}

We can use Theorem 15.1 to prove the existence of a weakly separable function. Using this theorem we construct a suitable weakly separable function in order to prove the following main theorem.

Theorem 15.2: Suppose $D=\left\{\left(c_{i}, x^{i}, y_{i}\right)\right\}_{i \in I}$ is a finite data set, where $c_{i}: X_{1} \times X_{2} \rightarrow \mathbb{R}_{+}$. Consider the following conditions:

(i) There exists a function $g\left(x_{1}, h\left(x_{2}\right)\right) \in \mathcal{T}_{p}^{a}(D)$, which is weakly separable in. $X_{2}$ and such that $g\left(x_{1}, \eta\right)$ is increasing ing $\eta_{\text {. }}$

(ii) There exists a function $g\left(x_{1}, h\left(x_{2}\right)\right) \in \mathcal{F}_{p}^{o}(D)$, which is weakly separable in $X_{2}$ and such that $g\left(x_{1}, \eta\right)$ is strictly increasing in $\eta$.

(iii) There exists a preorder $\succ$, on $\left\{x_{2}^{i}\right\}_{\text {BeI }}$ such that

$$
\begin{array}{ll}
y_{j}-c_{i}\left(x_{1}^{j}, x_{2}^{k}\right) \geq y_{i}-c_{i}\left(x^{i}\right) \Rightarrow x_{2}^{k} \precsim x_{2}^{j} & (k, i, j \in I), \\
y_{j}-c_{i}\left(x_{1}^{j}, x_{2}^{k}\right)>y_{i}-c_{i}\left(x^{i}\right) \Rightarrow x_{2}^{k} \prec x_{2}^{j} & (k, i, j \in I) .
\end{array}
$$

(iv) There exists a weakly separable function $g\left(x_{1}, h\left(x_{2}\right)\right) \in \mathcal{F}_{p}^{o}(D)$, such that $h$ and $g$ are strictly monotonically increasing continuous functions, and furthermore $h$ is concave and $g\left(\cdot h\left(x_{2}\right)\right)$, for every fixed $x_{2} \in X_{2}$, quasi-concave.

\section{Then:}

(a) (ii) $\Rightarrow$ (iii) $\Rightarrow$ (i).

(b) Suppose every function

$$
h\left(x_{2}\right)=\min _{i, j \in I} \alpha_{i j}+\lambda_{i} c_{i}\left(x_{1}^{j}, x_{2}\right) \quad\left(x_{2} \in X_{2}\right)
$$

where $\alpha_{i j} \in \mathbb{R}$ and $\lambda_{i}>0$, is such that the functions

$$
\bar{c}_{i}\left(x_{1}, \eta\right)=\inf _{h\left(x_{2}\right) \geq \eta} c_{i}\left(x_{1}, x_{2}\right) \quad\left(i \in l, x_{1} \in X_{1}, \eta \in \mathbb{R}\right),
$$

are strictly increasing in $\eta$. Then (iii) $\Leftrightarrow$ (iil).

(c) Suppose $D$ is a price and quantity data set. Then (ii) $\Leftrightarrow$ (iii) $\Leftrightarrow$ (iv).

Proof (a) (ii) $\Rightarrow$ (iii): Suppose $f(x)=g\left(x_{1}, h\left(x_{2}\right)\right) \in \mathcal{F}_{p}^{o}(D)$ is weakly separable as in (ii). Let $\dot{i}, j, k \in I$ and suppose

$$
y_{j}-c_{i}\left(x_{1}^{j}, x_{2}^{k}\right) \geq y_{i}-c_{i}\left(x^{i}\right) \text {. }
$$

Since $f \in F_{p}^{o}(D)$, one has moreover 


$$
y_{i}-c_{i}\left(x^{i}\right) \geq f\left(x_{1}^{j}, x_{2}^{k}\right)-c_{i}\left(x_{1}^{j}, x_{2}^{k}\right)
$$

Hence $y_{j}=f\left(x^{j}\right) \geq f\left(x_{1}^{j}, x_{2}^{k}\right)$, which inplies $h\left(x_{2}^{k}\right) \leq h\left(x_{2}^{j}\right)$, because $f(x)=g\left(x_{2}, h\left(x_{2}\right)\right)$ and $g\left(x_{1}, \eta\right)$ is strictly increasing in $\eta$. In a similar way one can prove

$$
y_{j}-c_{i}\left(x_{1}^{j}, x_{2}^{k}\right)>y_{i}-c_{i}\left(x^{i}\right) \Rightarrow h\left(x_{2}^{k}\right)<h\left(x_{2}^{j}\right) \quad\left(k, i_{j} j \in I\right)
$$

This ylelds (iii), using the weak order $\prec, \sim$ on $\left\{x_{2}^{i}\right\}_{\text {iel }}$ induced by $h$.

(iii) $\Rightarrow$ (i): Put

$$
z_{j}\left(x_{2}\right)=\min _{i \in I} y_{i}-c_{i}\left(x^{i}\right)-y_{j}+c_{j}\left(x_{1}^{j}, x_{2}\right) \quad\left(x_{2} \in X_{2}, i \in I\right)
$$

firom (iii) it follows that we may use Theorem 2.4 to construct a function

$$
h\left(x_{2}\right)=\min _{i \in I} h_{i}+\lambda_{i} x_{i}\left(x_{2}\right) \quad\left(x_{2} \in X_{2}\right)
$$

such that $\lambda_{i}>0$ and $h\left(x^{i}\right)=h_{i}$ for all $i \in I$. Now, let the transformed functions $\boldsymbol{T}_{h} c_{i}$ be defined as in Theorem 15.1

$$
\left(T_{h} c_{i}\right)\left(x_{1}, x_{2}\right)=\inf _{h(v) \geq h\left(x_{2}\right)} c_{i}\left(x_{1}, v\right) \quad\left(x_{1} \in X_{1}, x_{2} \in X_{2}, i \in I\right) .
$$

The construction of $h$ implies

$$
h\left(x_{2}\right) \geq h\left(x_{2}^{j}\right) \Rightarrow z_{j}\left(x_{2}\right) \geq 0 \quad\left(x_{2} \in X_{2}, j \in I\right),
$$

which means that one has

$$
y_{i}-c_{i}\left(x^{i}\right)-y_{j}+\left(\boldsymbol{T}_{h} c_{i}\right)\left(x^{j}\right) \geq 0 \quad(i, j \in I) .
$$

Hence $\left(\boldsymbol{T}_{h} c_{i}\right)\left(x^{i}\right) \geq c_{i}\left(x^{i}\right)$ and thus $\left(\boldsymbol{T}_{h} c_{i}\right)\left(x^{i}\right)=c_{i}\left(x^{i}\right)$, for all $i \in I_{y}$, because $\boldsymbol{T}_{h} c_{i} \leq c_{i}$ by definition of $\boldsymbol{T}_{h}$. Now, we have

$$
y_{j}-\left(\boldsymbol{T}_{h} c_{i}\right)\left(x^{j}\right) \leq y_{i}-\left(T_{h} c_{i}\right)\left(x^{i}\right) \quad(i, j \in I)
$$

thus by Theorem 13.1 we have moreover $\mathcal{F}_{p}^{\circ}\left(D^{\prime}\right) \neq \varnothing$ in Theorem 15.1. This means that condition (ii) in Theorem 15.1 is satisfied, which implies the existence of a weakly separable profit maximizing function $f=T_{h} f \in \mathcal{F}_{p}(D)$. Note that the resulting function in the proof of Theorem 15.1 is

$$
f(x)=\min _{i \in I} y_{i}+\left(\boldsymbol{T}_{h} c_{i}\right)(x)-\left(\boldsymbol{T}_{h} c_{i}\right)\left(x^{i}\right) \quad(x \in X) .
$$

(b) (iii) $\Rightarrow$ (ii): The functions $\boldsymbol{T}_{h} c_{\mathfrak{i}}$ in the above proof are weakly separable with aggregator function $\bar{c}_{i}\left(x_{1}, \eta\right)$. If the assumption in (b) is satisfied, then all these aggregator functions are strictly increasing in $\eta$. In that case the above constructed weakly separable function $f \in \mathcal{F}_{p}^{\circ}(D)$ will have the same property.

(c): Theorem 4.8 shows that the assumption made in (b) is satisfied by price and quantity data. "To prove (iii) $\Rightarrow$ (iv), we may inspect the weakly separable function $f$ that is constructed in the proof of (a) (iii) $\Rightarrow$ (i). For price and quantity data it will have the properties mentioned in (iv).

Finally, the implication (iv) $\Rightarrow$ (ii) is Irivial.

Condition (iii) in the above theorem may be used to test the hypothesis of weakly separable profit maximization. This condition is of the revealed. preference type and may be easily tested. In the same way as I remarked in the previous chapter, it has no sense to use this condition to test whether the Dutch industry data are consistent with weakly separable profit maximization. The data fitted so badly with the hypothesis of profit. maximization in Chapter 13 , that no valuable results can be expected for weakly separable profit maximization.

Varian (1984, Theorem 9) states weaker necessary and sufficient conditions concerning weakly separable profit maximization. However, these conditions 
are only necessary conditions. Varian did forget to show in the sufficiency proof that his constructed production function satisfies $g\left(x_{1}^{i}, h\left(x_{2}^{i}\right)\right)=y_{i}$, or in the notation of Varian $(1984)-g\left(x^{i}, h\left(z^{i}\right)\right)=y^{i}$.

Varian clains that in case of price and quantity data weakly separable profit maximization is equivalent to concave weakly separable profit maximization. However, it seems that there is not sucli an equivalence. The results in Varian (1984) indicate that the conditions concerning concave weakly separable proft maximization for price and quantity data might be as follows.

Conjecture: Suppose $D=\left\{\left(p^{i}, x^{i}, y_{i}\right)\right\}_{\text {iel }}$ is a frite price and quantity data sett of producer behaviour, where $X=X_{1} \times X_{2}$. Then the following conditions are equivalent:

(i) There exists a concave weakly separable function $g\left(x_{1}, h\left(x_{2}\right)\right) \in F_{p}^{o}(D)$ such that $g$ and $h: X_{2} \rightarrow \mathbb{R}$ are concave and $g\left(x_{1}, \eta\right)$ is strictly increasing in $\eta$.

(ii) There exist numbers $v_{i}$ and $\mu_{i}>0$ such that

$$
\begin{aligned}
& y_{i} \leq y_{j}+p_{1}^{j}\left(x_{1}^{i}-x_{1}^{j}\right)+\left(v_{i}-v_{j}\right) / \mu_{j} \quad(i, j \in I), \\
& v_{i} \leq v_{j}+\mu_{j} p_{2}^{j}\left(x_{2}^{i}-x_{2}^{j}\right) \quad(i, j \in I) .
\end{aligned}
$$

Proof $($ i) $\Rightarrow$ (ii): It seems to me that this proof should be based on similar arguments as given in Diewert and Parkan (1978, 1985), where conditions concerning concave weakly separable demand are considered.

(iii) $\Rightarrow$ (i): Put

$$
\begin{aligned}
& g\left(x_{1}, v\right)=\min _{i \in I} y_{i}+p_{1}^{i}\left(x_{1}-x_{1}^{i}\right)+\left(v-v_{i}\right) / \mu_{i} \quad\left(x_{1} \in X_{1}, v \in h\left(X_{2}\right)\right)_{4} \\
& h\left(x_{2}\right)=\min _{i \in I} v_{i}+\mu_{i} p_{2}^{i}\left(x_{2}-x_{2}^{i}\right) \quad\left(x_{2} \in X\right) .
\end{aligned}
$$

It is now easy to show that for every $i \in I$ one has

$$
\begin{aligned}
& g\left(x_{1}^{i}, v_{i}\right)=y_{i}, h\left(x_{2}^{i}\right)=v_{i}, \\
& \begin{aligned}
g\left(x_{1}, h\left(x_{2}\right)\right)-p^{i} x & \leq y_{i}+p_{1}^{i}\left(x_{1}-x_{1}^{i}\right)+\left(h\left(x_{2}\right)-v_{i}\right) / \mu_{i}-p^{i} x \\
& \leq y_{i}+p_{i}^{i}\left(x_{1}-x_{1}^{i}\right)+p_{2}^{i}\left(x_{2}-x_{2}^{i}\right)-p^{i} x=y_{i}-p^{i} x^{i} .
\end{aligned}
\end{aligned}
$$

So $g\left(x_{1}, h\left(x_{2}\right)\right) \in \mathcal{F}_{p}^{o}(D)$, which is clearly a concave function such that $g$ and $h$ is concave. 


\section{INTRODUCTION}

\subsection{Static or Dynamic Producer Behaviour}

The producer theory presented in the parts "Producer Demand" and "Profit Maximization" is concerned with static optimization behaviour: all that counts are inputs and costs at a given moment. This static theory is not directly connected with the practical situation of a producer. A producer who is only focussed on the current moment, will not be taken serious as a rival. In practice a producer has to decide about current and future investments. He has to estimate his discounted profit flow to pay off his debits. The capital stock depends dynamically on past investments, which means that the current investments do not only contribute to current profit, but also to future profit. So in practice a producer is facing a dynamic optimization problem.

Does this mean that the static theory is worthless? Not if we are able to transform the dynamic producer problem into a static problem. This transformation is the central problem in Chapter 17 , which presents solutions for the user cost of capital. In Chapter 18 the particular case is considered where the discounted price of investment is exponentially decreasing. A second issue, how to generate the capital stocks from the past investments, is treated in Chapter 19 . The remaining part of this chapter is an introduction to the dymamic relation of the capital stock with investments and a specification of the dynamic producer problem.

\subsection{The Dynamic Optimization Problem}

In practice, a profit maximizing producer is optimizing over a long period. To take into account future cost and profit, be uses discounted cost and profit, which depends on a discount rate. Such a producer maximizes the total present value of profit

$$
\max \int_{0}^{\infty} P(K(t), I(t), t) \mathrm{e}^{-\sigma t} d t
$$

where $P$ is profit, $K$ is the capital stock, $I$ is the investment flow, and $r$ is the discount rate. The producer may choose his current and future investments $I(t)$, for $t \geq 0$, but he cannot change his investments $I(t)$, for $t<0$, made in the past. Furthermore, there is a given capital investment relation, which specifies the capital stock $K(\cdot)$ that belongs to investment flow $I(*)$.

\subsection{Survival and Retirement of Investment}

The lifetime of a new investment can be described by using a survival function $h(t)$, representing the fraction of a unit investment that survives after a time $t$. In the beginning a unit investment is totally available and in the long run there will be nothing left. So, a survival function is a. 
decreasing function from one to zero at $t \geq 0$, and we may write

$$
h(t)=1-\int_{0}^{t} p(\tau) d \tau \quad(t \geq 0)
$$

where the retrement distribution $p(t)$ is a probability distribution. This probability distribution describes the retirement flow of a unit investment after a time $t$. Retirement distributions for investments are usually borrowed from statistical theory. The parametric families of linear, normal, Weibull and logistic probability distributions are widely used.

Below I will describe a few examples, which are: the exponental survival function, rectangular survival functions and the family of gamma retirement distributions. These examples will be discussed in the following chapters, in which especially the gamma distributions will be investigated.

The exponential survival function is of the form

$$
h(t)=1-\int_{0}^{t} \lambda \mathrm{e}^{-\lambda \tau} d \tau=\mathrm{e}^{-\lambda t} \quad(t \geq 0)
$$

for which the retirement distribution is the exponential function $\lambda \mathrm{e}^{-\lambda t}$.

Another survival function is the rectangular survival function

$$
h(t)= \begin{cases}1 & (t \in[0, T)), \\ 0 & \text { (otherwise), }\end{cases}
$$

for which a new investment remains unchanged in his lifetime $T$. Using the function

$$
\mathbb{I}(t)= \begin{cases}0 & (t<0), \\ 1 & (t \geq 0),\end{cases}
$$

we may describe this survival function as

$$
h(t)=1-1(t-T)=1-\int_{0}^{t} \delta(\tau-T) d \tau \quad(t \geq 0) .
$$

The above retirement distribution $\delta(t-T)$ is defined by using the delta distribution $\delta(\cdot)$, which satisfies by definition

$$
\int_{-\infty}^{\infty} \delta(t) f(t) d t \equiv f(0)
$$

for every function $f$.

The gamma distribution is a probability distribution, determined by two parameters $\alpha$ and $\lambda$ and given by

$$
p(t)=\lambda^{\alpha} t^{\alpha-1} \mathrm{e}^{-\lambda t} / \Gamma(\alpha) \quad(t \geq 0)
$$

where

$$
\Gamma(\alpha)=\int_{0}^{\infty} t^{\alpha-1} \mathrm{e}^{-t} d t
$$

This family includes the exponential retirement distribution, for which one has $\alpha=1$. The family gamma distributions also approaches the delta distribution $\delta(t-T)$, which can be described as the limit for $\alpha \rightarrow \infty$ of gamma distributions with mean lifetime $T$. Examples of retirement distributions with $T=1$ are given in Figure 16.1, while Figure 16.2 displays the corresponding survival functions.

The Netherlands Central Bureau of Stratistics (1986) estimated the gamma retirement distribution for several types of capital assets in the Dutch 
industry. All estimated walues of $\alpha$ were found in the range $2 \leq \alpha \leq 3.5$. Thus one may doubt whether the widely used exponental survival function with $\alpha=1$, and the rectangular survival function with $\alpha=\infty$, are suitable choices.

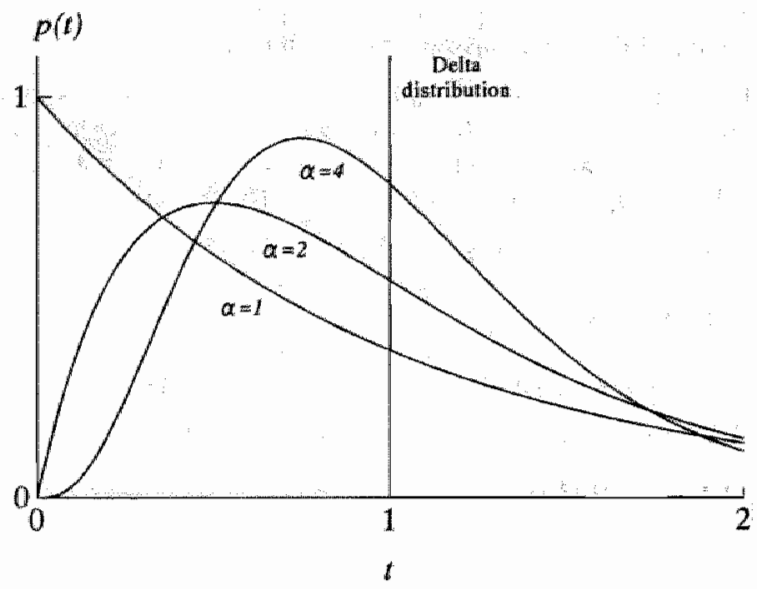

Figure 16.1 Retirement Distributions

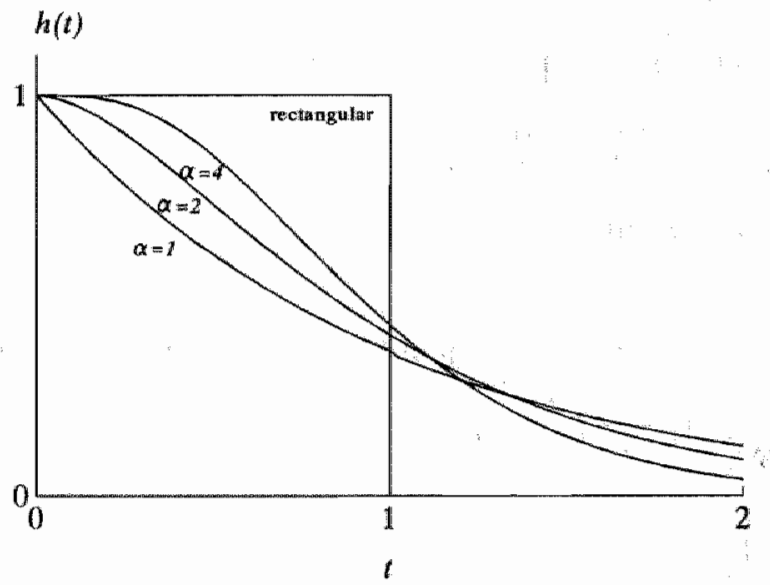

Figure 16.2 Surviwal Functions

\subsection{Capital Stocks}

A capital stock is dynamically related to current investments and investments made in the past. In this section I will specify this relation in a decreasing level of abstraction. A general description is $K=\boldsymbol{A} I$, where $\boldsymbol{A}$ is a 
limear operator. Such a linear operator maps each investment flow function $I(t)$ on the corresponding capital stock function $K(t)$.

Definition: An operator A is called lmear when it lias the following properties:

$$
\begin{aligned}
& A\left(I_{1}+I_{2}\right)=A I_{1}+A I_{2}, \\
& A \lambda I=\lambda A I \quad(\lambda \in \mathbb{R}) .
\end{aligned}
$$

Below I will consider examples of linear operators $A$, which describe the survival and retirement of investments.

Most empirical estimations of capital stocks are based on the perpetual inventory method, which determines the capital stock as a weighted sum of past gross investment flows. In a continuous time description we have then

$$
K(t)=\int_{-\infty}^{t} h(t-\tau) I(\tau) d \tau \quad(t \in \mathbb{R})
$$

where $h$ is a survival function for investments, described in the previous section. We may simplify the notation, by putting $h(t)=0$ for all $t<0$, which implies $h(t-\tau)=0$ for all $\tau>t$. In that case we may write

$$
K(t)=\int_{-\infty}^{\infty} h(t-\tau) I(\tau) d \tau \quad(t \in \mathbb{R})
$$

So, by using the convolution operator which is defined as

$$
f * g(t) \equiv \int_{-\infty}^{\infty} f(t-\tau) g(\tau) d \tau \quad(t \in \mathbb{R})
$$

we have

$$
K(t)=h * I(t) \quad(t \in \mathbb{R})
$$

The operator $A$ described by $A I=h * I$ is clearly an example of a linear operator.

Another way to describe the behaviour of the capital stock is

$$
\frac{d}{d t} K=I-B I
$$

where $B$ is a linear operator. The term $B I$ represents in this equation the flow of retired investments. Such a relation can be derived by using a retirement distribution $p$ that determines a survival function $h$ and the capital stock in the following way.

Theorem 16.1: Suppose $p$ is a retirement distribution and $p(t)=0$ for all $t<0$. Then the survival function

$$
h(t)=1-\int_{0}^{t} p(\tau) d \tau \quad(t \geq 0)
$$

can be written as $h=1(\cdot) *(\delta(\cdot)-p)$. Moreover, the capital stock $K=h$. I is gowerned by the equation.

$$
\frac{d}{d t} K=I-p * I
$$

where $p * I$ represents the flow of retired investments.

Proof: We have

$$
K=h * I=[1(\cdot) *(\delta(\cdot)-p)] * I=1(\cdot) *[(\delta(\cdot)-p) * I]=\int_{-\infty}^{t}(I-p * I) d \tau_{4}
$$

where the associative equality

$$
[1(\cdot) *(\delta(\cdot)-p)] * I=1(\cdot) *[(\delta(\cdot)-p) * I]
$$

follows from Fubini's theorem, assuming that 


$$
\int_{-\infty}^{t}|I| d r<\infty \quad(t \in \mathbb{R})
$$

and using the assumption that $p$ is a probability distribution.

Thus summarizing the above, four ways to describe the capital investment relation are

$$
K=A I, K=h \cdot, \frac{d}{d t} K=I-B I, \frac{d}{d t} K=I-p * I,
$$

where $A$ and $B$ are a linear operators, $h$ is a survival function and $p$ is a retirement distribution.

\subsection{Contents of Part IV}

Chapter 17 searches for the user cost of capital and considers several types of capital investment relations. Because this book is concerned with nonparametric theory, this chapter considers nonparametric defined relations. This complicates the problem at first sight, but using a mathematical machinery, distribution theory (e.g. Zemanian, 1965), simple elegant results can be derived. To keep the book readable the developed theory is roughly described without going into details. In Chapter 18 the particular case is considered in which the discounted price of investment is exponentially decreasing. If this assumption is satisfied, one may easilly derive the user cost of capital for any survival function. In that case it is not necessary to use the assumption that investments are exponentially decaying.

Another problem is the construction of the capital stocks. An easy way to construct capital stocks is considered in Chapter 19. Although this construction is based on a parametric specification, it will be shown that this specification is flexible enough to approach empirical capitall investment relations. 


\section{USER COST OF CAPITAL}

\subsection{Introduction}

Given the discounted price of the investments, what is the shadow price of capital? The determination of this price, the user cost of capitat, is the subject of this chapter. It is of importance, because it may transform the dymamic optimization problem of a producer into a static problem.

Computation of the user cost of capital is relatively simple in case of exponential and rectangular survival functions. That is a reason why these survival functions are popular in empirical models. Applications of dynamic optimization to exponential survival functions are made by Jorgenson (1967) and others. A rigorous description of Jorgenson's theory and several extensions may be found in Takayama (1974). Takayama considers constraints on investments and the effect of adjustment costs. The maximum principle can directly be applied in case the survival function is exponential, which is a reason why the exponential survival function is popular in economic theory.

However, one may ask whether the exponential and rectangular functions are suitable as survival function for empirical investment data. The Netherlands Central Bureau of Statistics (1986) investigated the form of survival functions for several capital goods in the Dutch industry, and their results deviate clearly from the exponential and rectangular form.

In this chapter I will derive the user cost of capital for survival functions of a general form. For a complete theory there are two aspects that have to be treated: the determination of the user cost of capital and the generation of capital stocks. The latter will be treated in Chapter 19.

\subsection{A General User Cost of Capital Solution}

Suppose there is a linear relation between capital $K(t)$ and investment $I(t)$, of the form $K=A I$, where $A$ is a linear operator. This operator maps each admissible investment function $I(\cdot)$ on a capital stock function $K(\cdot)$. I use the vague term 'admissible' to avoid the introduction of restrictions, necessary for a rigorous description, but not necessary to explain the essential theory.

Before I derive the solution of the user cost of capital, I have to describe the cost of investments. This is

$$
\int_{0}^{\infty} p_{I}(t) l(t) d t
$$

which represents the present value of investment costs, using discounted investment price $p_{I}$. When we define the innerproduct $\left\langle v_{3} w\right\rangle$ of two functions $v$ and w as

$$
\langle v, w\rangle \equiv \int_{-\infty}^{\infty} v(t) w(t) d t
$$

and we put $p_{I}(t)=0$ for all $t<0$, the present value of the investment cost can be writien as

$$
\left\langle p_{I}, I>\equiv \int_{-\infty}^{\infty} p_{I}(t) I(t) d t\right.
$$

Now, the user cost of capital $\tilde{p}_{K}$, given a discounted investment price 
function $p_{I}$, has to satisfy

$$
\left\langle p_{r}, I\right\rangle=\left\langle p_{K}, K\right\rangle \quad(K=\mathbb{A})
$$

for every admissible investment flow $I$.

To derive the user cost of capital the following idea is crucial.

Definition: Given a linear operator $L$ and an innerproduct $\langle;$,$\rangle , the$ adjoint operator $L^{\dagger}$ is the linear operator that satisfies

$$
<L v, w>=\langle v, L w>
$$

for all admissible functions $v$ and $w$.

Example 17.1 A simple example is the case in which $L$ is a matrix and the vector innerproduct is $\langle v, w\rangle=v v^{t}$. In that case one has $L^{\dagger}=L^{t}$, because

$$
\left\langle L^{t} v, w\right\rangle=\left(L^{t} w\right)^{t} w=v^{t} L w=\langle v, L w\rangle \text {. }
$$

Example 17.2 For the linear operator $L=\frac{d}{d t}$ with innerproduct

$$
<v, w>=\int_{-\infty}^{\infty} v(t) w(t) d t
$$

we have

$$
\langle L v, w\rangle+\left\langle v_{y} L w\right\rangle=\int_{-\infty}^{\infty} \frac{d}{d t}[v w] d t=\left.v w\right|_{-\infty} ^{+\infty}=0 .
$$

Where the last equality follows by supposing that $v(t)$ u( $(t)$ vanishes for $t \rightarrow \pm \infty$. We have then

$$
\langle v, L w\rangle=\langle-L v, w\rangle=\left\langle L^{4} w, w\right\rangle
$$

and

$$
\left[\frac{d}{d t}\right]^{\dagger}=\frac{d}{d t}
$$

Now, suppose we can reconstruct the investment function $I(\cdot)$ from the capital stock function $K(\cdot)$. In other words: there is an inverse linear operator $A^{-1}$ with the property $I=A^{-1} A I=A^{-1} K$.

Theorem 17.1: Suppose $A$ is linear operator and there is an anverse operator $A^{-1}$ that satisfies $I=A^{-1} A I=A^{-1} K$. Then the user cost of capital for the problem

$$
\left\langle p_{I}, I\right\rangle=\left\langle p_{K}, K\right\rangle \quad(K=A I)
$$

8

$$
p_{K}=\left(A^{-1}\right)^{\dagger} p_{\Gamma}
$$

Proof: For the user cost of capital we have

$$
\left\langle p_{l}, I\right\rangle=\left\langle p_{I}, A^{-1} K\right\rangle=\left\langle\left(A^{-1}\right)^{*} p_{I}, K\right\rangle=\left\langle p_{K}, K\right\rangle .
$$

With the above theoretical outline we can solve the user cost of capital for the textbook assumption of exponential decay.

Example 17.3 We have for survival function $\mathrm{e}^{-\lambda t}$ the relation.

$$
\frac{d}{d t} K=I-\lambda K \text {. }
$$

From this we obtain

$$
I=\left[\lambda+\frac{d}{d t}\right] K \text {. }
$$

and the user cost of capital $p_{K}$ is thus

$$
\left.p_{K}=\left[\lambda+\frac{d}{d t}\right]^{\dagger} p_{X}=\llbracket \lambda-\frac{d}{d t}\right] p_{I}
$$


using $\lambda^{\|}=\lambda$ and the relation

$$
\left[\frac{d}{d t}\right]^{\dagger}=\frac{d}{d t}
$$

given in Example 17.2, which is valid when we assume that $p_{I}(t) I(t)$ vanishes for $t \rightarrow+\infty$. Note that $p_{r}(t) /(t)$ vanishes for $t \rightarrow-\infty$, since we suppose $p_{X}(t)=0$ for all $t<0$.

\subsection{User Cost of Capital for Survival Funetlons}

With distribution theory (e.g see Zemanian, 1965) one can derive the user cost of capital when a survival function $h$ is given. In that case capital is given by the equation

$$
K=h * I=\int_{-\infty}^{\infty} h(t-\tau) I(\tau) d \tau .
$$

I will solve the user cost of capital using Laplace transforms.

Definition: The Laplace transform of a function $f(\cdot)$ is defined as

$$
\mathcal{L}[f](s)=\int_{-\infty}^{\infty} f(t) \mathrm{e}^{-s t} d t \quad(s \in \mathbb{C})
$$

For Laplace transforms I use the following short hand notation

$$
\tilde{f} \equiv \mathcal{L}[f] \text {. }
$$

The Laplace transform has useful properties from which the user cost of capital can be derived.

Theorem 17.2: Suppose $h$ is a survival function. Then the user cost of capital for the problem

$$
<p_{T}, I>=\left\langle p_{K}, K>\quad(K=h * I)\right.
$$

is

$$
p_{K}=\mathcal{L}^{-1}\left[\tilde{p}_{I}(s) / \tilde{h}(-s)\right]
$$

Proof: Let $L$ be the linear operator such that $L f=h * f$, for every admissible function $f$. Now, the distribution theory derives, under weak conditions, the equalities

$$
\mathcal{L}[L f]=\tilde{h} \tilde{f}, \quad \mathcal{L}\left[L^{-1} f\right]=\tilde{f} / \tilde{h}, \quad \mathcal{L}\left[L^{\dagger} f\right]=\tilde{\tilde{h}} \tilde{f},
$$

where the notation

$$
\tilde{h}(s)=\tilde{h}(-s) \quad(s \in C)
$$

is used. Using the above three properties, we may obtain the Laplace transform of the user cost of capital $p_{K}$. Concerning $A I=h_{*} I$ in Theorem 17.1, these properties imply

$$
\tilde{p}_{K}(s)=\mathcal{L}\left[\left(\boldsymbol{A}^{-1}\right)^{H} p_{T}\right]=\tilde{p}_{I}(s) / \tilde{h}(-s) \text {. }
$$

Now, using the inversion $\mathcal{C}^{-1}$ of the Laplace transform, we derive the given itser cost of capital.

Example 17.5. Let us consider the simple case of the exponential survival function

$$
h(t)=1(t) \mathrm{e}^{-\lambda t},
$$

where 


$$
\mathbb{I}(t)= \begin{cases}0 & (t<0) \\ 1 & (t \geq 0) .\end{cases}
$$

In that case we have $(s)=(A+s)^{-1}$ and we obtain

$$
p_{K}=\mathcal{L}^{-1}\left[\tilde{p}_{Y}(s) / \tilde{h}(-s)\right]=\mathcal{L}^{-1}\left[\tilde{p}_{I}(\lambda-s)\right]=\left(\lambda-\frac{d}{d t}\right) p_{I}
$$

\subsection{User Cost of Capital for Retirement Distributions}

Giwen the retirement distribution for investment $p(t)$, the capital stock is govened by the equation

$$
\frac{d}{d t} K=I-B I=(1-B) I
$$

where $B I=p+$. We may apply now Theorem 17.11 to derive the user cost of capitial.

Theorem 17.3: Suppose $B$ is a linear operator. Then the user cost of capital for the problem

$$
\left\langle p_{I}, I\right\rangle=\left\langle p_{K}, K\right\rangle \quad\left(\frac{d}{d t} K=I-B I\right)
$$

is

$$
p_{K}=-\frac{d}{d t} \sum_{n=0}^{\infty}\left(B^{\dagger}\right)^{n} p_{I}
$$

where $\left(B^{\dagger}\right)^{0}=1,\left(B^{\dagger}\right)^{2}=B^{\dagger} B^{\dagger},\left(B^{\dagger}\right)^{3}=B^{\dagger} B^{\dagger} B^{\dagger}$, etc.

Proof: We have

$$
I=(1-B)^{-1} \frac{d}{d t} K
$$

The inverse linear operator $(\mathbb{1 - B})^{-1}$ can be represented by using Neumann senties:

$$
(1-B)^{-1}=\sum_{n=0}^{\infty} \boldsymbol{B}^{n}
$$

where $\boldsymbol{B}^{0}=\mathbf{1}, \boldsymbol{B}^{2}=\boldsymbol{B B}, \boldsymbol{B}^{3}=\boldsymbol{B} \boldsymbol{B} \boldsymbol{B}$, etc. Note that this solution is analogous to the solution in the case that $B$ is a scalar. Now, we may apply Theorem 17.1 to derive

$$
\begin{aligned}
\left\langle p_{K}, K\right\rangle & =\left\langle p_{I},(1-B)^{-1} \frac{d}{d t} K>=\left\langle p_{I}, \sum_{n=0}^{\infty} B^{n} \frac{d}{d t} K\right\rangle=\left\langle\frac{d^{\dagger}}{d t} \sum_{n=0}^{\infty}\left(B^{n}\right)^{\dagger} p_{Y}, K\right\rangle=\right. \\
& \left.=<-\frac{d}{d t} \sum_{n=0}^{\infty}\left(B^{\dagger}\right)^{n} p_{I}, K\right\rangle .
\end{aligned}
$$

Now, ut remains to solve the operator $B^{t}$ in case $B$ is a linear operator of the form $B w=p_{*} w$. Using the notation

$$
\breve{p}(t) \equiv p(-t) \quad(t \in \mathbb{R}),
$$

the solution of this problem is $\boldsymbol{B}^{\dagger} w=\ddot{p}_{w} w$. Using this expression in Theorem 17.3 solves the user cost of capital in case a retirement distribution is given.

Theorem 17.4: Suppose $p$ is a retimement distribution. Then the user cost of capital for the problem

$$
\left\langle p_{I}, I\right\rangle=\left\langle p_{K}, K\right\rangle \quad\left(\frac{d}{d t} K=I-p_{*} I\right)
$$




$$
p_{K}=\frac{d}{d t} \sum_{n=0}^{\infty}(\breve{p})^{n}+p_{I} \quad(t \geq 0)
$$

where $(\breve{p})^{0}=\delta(\cdot)$ and $(\check{p})^{n+1}=\breve{p} *(\breve{p})^{n}$.

Proof: Fubini's theorem implies the equality $\langle v, p * w\rangle=\left\langle\tilde{p}_{*}, w, w\right\rangle$, when the weak condition $<|\vartheta|,|p| w|w|><\infty$ is satisfied. Hence, the result follows from Theorem 27.3.

Example 17.6 As an example I will apply the above equation, which describes the user cost of capital $p_{K}$, to the rectangular survival function

$$
h(t)=1-\int_{0}^{t} \delta(\tau-T) d \tau \quad(t \geq 0)
$$

where the retirement distribution is $p(t)=\delta(t-T)$. Hence

$$
(\check{p}(t))^{n}=\delta(t+n T) \quad(n \in \mathbb{N}),
$$

and from the given equation for the user cost of capital, we derive

$$
p_{K}(t)=\frac{d}{d t} \sum_{n=0}^{\infty}(\check{p})^{n} p_{I}=\frac{d}{d t} \sum_{n=0}^{\infty} p_{I}(t+n T) \quad(t \geq 0)
$$

\subsection{Relation with Measure Theory}

In the above sections the user cost of capital is derived with a direct approach. An indirect way to derive the user cost of capital is the following use of measure theory. Suppose the capital investment relation $K=A I$ is invariant under time translation. With the latter I. mean

$$
A T_{a} I=T_{a} A I \quad(a \in \mathbb{R})
$$

where translation operator $T_{a}$ is defined as

$$
\left(T_{a} f\right)(t)=f(t+a) \quad(t, a \in \mathbb{R})
$$

for arbitrary functions $f$.

Remark: In quantum mechanics there is a symmetry for a linear operator $A$ when there is a linear operator $\boldsymbol{B}$ such that $[\boldsymbol{A}, \boldsymbol{B}] \equiv \boldsymbol{A} \boldsymbol{B}-\boldsymbol{B A}=\mathbf{0}$. This is the case for the translation operator $B=T_{a}$.

A descriptive rule to solve the user cost of capital is now the following. First solve the investment flow $I_{1}$ that generates one unity of capital of an infinite lifetime, thus $\boldsymbol{A} I_{1}=1(\cdot)$. Then we may obtain the user cost of capital from the following theorem.

Theorem 17.5. Suppose $A$ is a limear operator, invariant under time translation, and $\mathrm{AI}_{1}=1(\cdot)$. Then the user cost of capital for the problem

$$
\left\langle p_{I}, I\right\rangle=\left\langle p_{K}, K\right\rangle \quad(K=A I)
$$

is

$$
p_{K}=-\frac{d}{d t} \check{I}_{1} * p_{T}
$$

where we use the notation

$$
\check{I}_{1}(t)=I_{1}(-t) \quad(t \in \mathbb{R}) .
$$

Proof: The equality $A I_{1}=1(\cdot)$ and $A$ translation invariant means that we have

$$
\boldsymbol{T}_{\mathrm{t}}, 1(\cdot)=\boldsymbol{T}_{t} \boldsymbol{A} I_{\mathbb{1}}=\boldsymbol{A} \boldsymbol{T}_{t} I_{1} \quad(\mathfrak{t} \in \mathrm{R})
$$

where $T_{t}$ is the translation operator. Because the user cost of capilal $p_{k}$, given a function $p_{I}$, satisfies $\left\langle p_{K}, A I\right\rangle=\left\langle p_{I}, I\right\rangle$, we have thus 
146 Capital

$$
\left\langle p_{K}, A T_{t} I_{1}\right\rangle=\left\langle p_{K}, T_{t} 1(\circlearrowleft)=\left\langle p_{T} T_{t} I_{1}\right\rangle \quad(t \in \mathbb{R})\right.
$$

So, we have

$$
\begin{aligned}
p_{K}(t) & =\frac{d}{d t} \int_{t}^{\infty} p_{K}(\tau) d \tau=\frac{d}{d t}\left\langle p_{K}, T_{t} 1()\right\rangle \\
& \left.=\frac{d}{d t}<p_{I}, T_{t} I_{1}\right\rangle=\frac{d}{d t}\left(I_{1} * p_{X}\right)(t) \quad(t \in R) .
\end{aligned}
$$

From the above equation for the user cost of capital $p_{K}$ and the result in the previous section, which was

$$
\frac{d}{d t} K=I-p=I \Rightarrow p_{K}=-\frac{d}{d t} \sum_{n=0}^{\infty}(\breve{p})^{n} p_{I}, \text { where }(\breve{p})^{0}=\delta(\cdot)(\breve{p})^{n+1}=\breve{p}_{*}(\check{p})^{n}
$$

we may expect that we have

$$
I_{1}=\sum_{n=0}^{\infty}(\ddot{p})^{n} \text {. }
$$

This conjecture is indeed correct as is shown below.

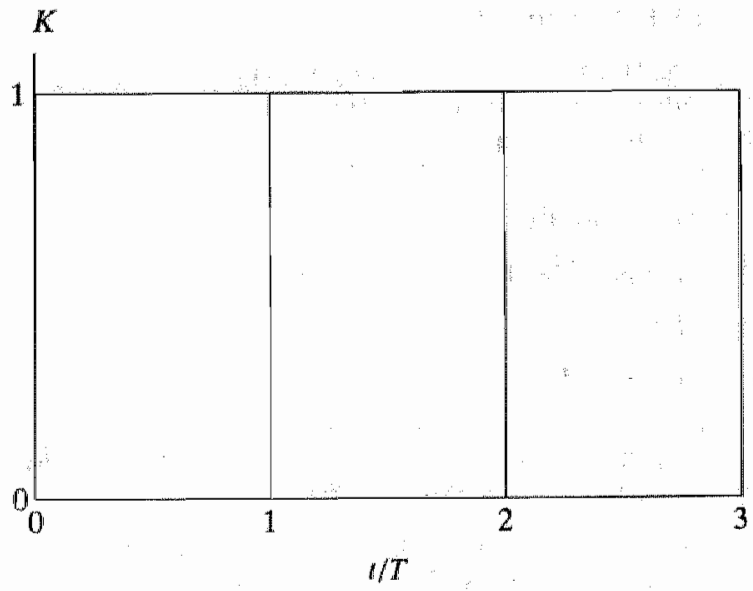

Figure 17.1 Capital Rectangular Function

Theorem 17.6: The equation

$$
\frac{d}{d t} 1(\cdot)=I_{\mathbb{H}}-p_{*} I_{1}
$$

iss solved by

$$
\check{I}_{1}=\sum_{n=0}^{\infty}(\breve{p})^{n}
$$

Proof: For this solution one has

$$
I_{1}=\sum_{n=0}^{\infty} p^{n}
$$

and 


$$
\frac{d}{d t} 1(\cdot)=\delta(\cdot)=p^{0}=\sum_{n=0}^{\infty} p^{n}-p * \sum_{n=0}^{\infty} p^{n}=I_{1}-p * I_{1} .
$$

In the following example the results are applied to the rectangular survital function.

Example 17.6 To build a unit capita] stock of an infinite lifetime, using a rectangular survival function, we have to repeat our investment after the lifetime $T$. This is displayed in Figure 17.1. Thus the investment flow $I_{1}$ consists of a series delta distributions and is of the form

$$
l_{1}(t)=\sum_{n=0}^{\infty} \delta(t+n T) \quad(t \in \mathbb{R}) .
$$

I will now use the above method to clarify why future investment prices play no part in the user cost of capital in case of exponentially decaying investments. As I will show below, this is caused by the fact that new and partly decayed investments are indistinguishable in the case of exponential decay.

Example 17.7 let the capital investment relation be given as

$$
\frac{d}{d t} K=I-\lambda K, K(-\infty)=0
$$

To build capital $K(t)=\mathbb{1}(t)$, we may use the investment flow

$$
I_{1}(t)=\delta(t)+\lambda \Perp(t)
$$

because we have then

$$
\frac{d}{d t} K=\frac{d}{d t} 1(t)=\delta(t)=\delta(t)+\lambda 1(t)-\lambda 1(t)=I_{1}-\lambda \mathbb{1}(t)=I_{1}-\lambda K .
$$

So, the given capital investment relation

$$
\frac{d}{d t} K=I_{1}-\lambda K, K(-\infty)=0
$$

is satisfied. The above derived user cost of capital solution is thus

$$
\begin{aligned}
p_{K} & \left.=-\frac{d}{d t} p_{I} \check{L}_{1}=-\frac{d}{d t}<p_{I}, T_{t}[\delta(\cdot)+\lambda \mathbb{L}(\cdot)]\right\rangle \\
& =-\frac{d}{d t}\left[p_{I}+\int_{t}^{\infty} p_{I}(\tau) \lambda d \tau\right]=-\frac{d}{d t} p_{I}+\lambda p_{I} .
\end{aligned}
$$

The future investment price are of no importance in this example, because there is no difference between new and decayed investments in case of exponentially decaying investments. This leads to the constant weight measure $\lambda$ that appears in the formula

$$
\int_{t}^{\infty} p_{I}(\tau) \lambda d \tau
$$

which is differentiated in order to compute $p_{K}$. Only because the weight measure $\lambda$ is constant in this formula, the future investment prices $p_{I}(t)$, $\tau>t$, have no chance to play a part in the user cost of capital $p_{K}(t)$.

\subsection{A Generalization}

One may generalize the theory in this chapter in case investment cost is noninear. In that case one should use a dynamic multiplier in a similar way as in the maximum principle of Pontryagin (1962). For general forms of survival functions we cannot use the standard theory, but we may apply the following modified approach.

The general problem is 


$$
\max \int g(K, I, t) d t
$$

where $K=A l$. To convert this problem to an unconstrained maximization problem, we adjoin this constraint using a dynamic Lagrange multiplier $\eta$. Now, we obtain

$$
\max _{K, I} \int[g-\eta(K-A I)] d t=\max _{K} \int\left[g-\eta K+\left(A^{\dagger} \eta\right) I\right] d t
$$

The latter integral may be maximized over $I$ and $K$ before integration, which implies the first order conditions

$$
\frac{\partial}{\partial T} g+A^{\dagger} \eta=0, \frac{\partial}{\partial R} g-\eta=0
$$

So, a solution of the optimization problem has to satisfy the restriction

$$
\frac{\partial}{\partial T} g+A^{\prime}\left(\frac{\partial}{\partial R}\right)=0
$$

In this chapter the case was investigated in which $g$ is linear in $I$. In that case $g$ is of the form $g(K, I, t)=f(K, t)-p_{I}(t) I$. Hence, we have

$$
\frac{\partial}{\partial I} g=-p_{I}
$$

and we cain solve the dynamic Lagrange multiplier $\eta$ from

$$
-p_{I}+\boldsymbol{A}^{\dagger}\left(\frac{\partial}{\partial K} g\right)=0 \Rightarrow \eta=\frac{\partial}{\partial K} g=\left(\boldsymbol{A}^{\dagger}\right)^{-1} p_{I^{*}}
$$

This means that we are left with the maximization problem

$$
\max _{K} f(K, t)-j K, \eta=\left(A^{\sharp}\right)^{-1} p_{I},
$$

where $\eta$ represents the user cost of capital.

Another case of interest is the case in which $g$ is linear in $K$. In that case we have $g(K, I, t)=p_{K}(t) K-c(I, t)$, where $p_{K}$ represents the discounted marginal revenue of capital. Now, we have

$$
\eta=\frac{\partial}{\partial K} g=p_{K}
$$

and we are left with the following maximization problem

$$
\max _{I}\left(A^{\dagger} \eta\right) I-c(I, t), \eta=p_{K}
$$

where $\left(A^{\dagger} \eta\right) I$ is the discounted revenue of investment and $c$ the discounted investment cost.

\subsection{Summary and Conclusions}

The basis of the theory concerning the user cost of capitall is the capital investment relation $K=\boldsymbol{A} I$, where $\boldsymbol{A}$ is linear operator that maps investment flow $I(\cdot)$ on a capital stock $K(\cdot)$. With the idea of the user cost of capital we may climinate the investment flow $I(*)$ from a given dynamic optimization problem of the form

$$
\max _{I} \int_{0}^{\infty}\left[f(k, t)-p_{I}(t) I\right] d t
$$

where $K=\boldsymbol{A}$. This elimination transforms the dynamic optimization problem into a static problem of the form

$$
\max _{K} \int_{0}^{\infty}\left[f\left(K_{n}, t\right)-p_{K}(t) K\right] d t
$$

which contains only the capital stock $K$ as a variable. Now, we may optimize before integration in contrast to the original dynamic problem. Furthermore, we may use the relation $I=A^{-1} K$ to derive the optimal investment flow from the optimal capitall stock solution. 
Below a short summary of the results is given. Suppose the relation of the capital stock $K$ with investment $I$ is described, using a linear operator $A$, a survival function $h$, and a retirement distribution $p$ respectively. In that case we obtain a corresponding relation between the user cost of capital $p_{k}$ and the discownted investment price $p_{l}$. In this chapter the following rellations are derived:

$$
\begin{aligned}
& K=A I \Rightarrow p_{K}=\left(A^{-1}\right)^{*} p_{I} \\
& K=h * I \Rightarrow p_{K}=\mathcal{L}^{-1}\left[\tilde{p}_{I}(s) / \tilde{h}(-s)\right],
\end{aligned}
$$

where $\tilde{f}=\mathcal{L}(f)$ denotes the Laplace transform of $f$.

Using a retirement flow and a linear operator $\boldsymbol{B}$, we have derived.

$$
\frac{d}{d t} K=I-B I \Rightarrow p_{K}=\frac{d}{d t} \sum_{n=0}^{\infty}\left(B^{\dagger}\right)^{n} p_{I} \text {, where }\left(B^{\dagger}\right)^{0}=1,\left(B^{\dagger}\right)^{n+1}=B^{\dagger}\left(B^{\dagger}\right)^{n}
$$

In case of a retirement distribution $p$, we have derived

$$
\frac{d}{d t} K=I-p_{*} I \Rightarrow p_{K}=-\frac{d}{d t} \sum_{n=0}^{\infty}(\check{p})^{n} * p_{I}, \text { where }(\check{p})^{0}=\delta(\cdot),(\check{p})^{n+1}=\breve{p} *(\check{p})^{n+}
$$

where the notation

$$
f(t)=f(-t): \quad(t \in \mathbb{R})
$$

is used. A result that is related to the latitier result is

$$
K=A I, A \text { time-independent, } A I_{1}=1(\cdot) \Rightarrow p_{K}=\frac{d}{d t} \breve{I}_{1} * p_{I} \text {. }
$$

What is the global lesson we can learn from the above theory? In the standard case in literature, where the investment surviwal function is of the exponential form, the current user cost of capital depends on the current discounted investment price and its derivative. This allows a myopic producer to be an optimizer. However, in general an optimizing producer has to look forward in the future. Concerning surwival functions that are not of the exponential form, the user cost of capital depends on futtre values of the discounted investment price.

One may generalize the theory in this chapter in order to apply it to nonlinear investment costs. In that case one should use dynamic multipliers in a sinilar way as in the maximum principle of Pontryagin (1962).

Finally, I have to make a remark about the theory in this chapter. I have not considered restrictions on the investment flow. For example, it may be required that investments are positive. The case in which such a restriction becomes active is not covered by the given theory. 


\section{EXPONENTIAL DISCOUNTED INVESTMENT PRICE}

\subsection{Introduction}

In the literature one often uses exponentially decreasing discounted inwestment prices. In this chapter wre shall solve the user cast of capilal for this particular case. The given solution may be applied to any investment capital relation that is derived from a survival function. I have used the yesults in this chapter to compute the user cost of capital for the Dutch industry data. These data are used in this book in order to show applications of the developed producer theory.

\subsection{Solution for the Exponential Price}

Suppose the discounted investment price $p_{l}$ is of the form

$$
p_{I}(t)=p_{I}(0) e^{-r t} \quad(t \geq 0)
$$

giver a discount rate $r>0$. Civen an investment survival function $h$, the previous chapter states that the user cost of capital is equal to

$$
p_{R}=\mathcal{L}^{-\dot{1}}\left[\tilde{p}_{I} / \tilde{h}\right]
$$

This expression is the inversion of the laplace transform $\tilde{p}_{I} / \tilde{\tilde{h}}$, where $\tilde{p}_{I}$ and $\tilde{h}$ are the Laplace transforms of $p_{I}$ and $h$ respectively. For example, the Laplace transform $\tilde{h}$ of $h$ is defined as

$$
\tilde{h}(r)=\int_{-\infty}^{\infty} h(t) \mathrm{e}^{-r t} d t
$$

Furthermore, the notation $\tilde{h}(s)=\tilde{h}(-s)$, for $s \in \mathbb{C}$, is used.

Given the form of the discounted investment $p_{1}$, we may derive a simple expression for the user cost of capital. This expression is given in the following theorem.

Theorem 18.1: Suppose $h$ is a survival function and

$$
p_{I}(t)=p_{I}(0) e^{-r t} \quad(t \geq 0) .
$$

Then the user cost of capital for the problem.

$$
\left\langle p_{I}, I\right\rangle=\left\langle p_{K}, K>\quad\left(K=h_{*} I\right),\right.
$$

wh which we assume $I(t)=0$ for all $t<0$, is

$$
p_{k}=p_{r} / \tilde{h}(r)
$$

Proof: The assumption $h(t)=I(t)=0$ for all $t<0$, umplies $K(t)=\left(h^{*} I\right)(t)=0$ for all. $t<0$. Hence, we have $\left\langle\hat{p}_{I} I\right\rangle=p_{I}(0) \tilde{I}(r)$ and $\left\langle p_{I}, \tilde{K}\right\rangle=p_{I}(0) \tilde{K}(r)$. So, because by the convolution theorem given in Appendix $D$, we have

$$
K=h \cdot I \Rightarrow \tilde{K}(r)=\tilde{h}(r) \tilde{I}(r)
$$

we obtain

$$
\begin{aligned}
<p_{I}, I> & =p_{I}(0) \tilde{I}(r)=\tilde{h}(r)^{-1} p_{I}(0) \tilde{h}(r) \tilde{I}(r)=\tilde{h}(r)^{-1} p_{I}(0) \tilde{K}(r) \\
& =\left\langle\tilde{h}(r)^{-1} p_{I}, K>=\left\langle p_{K}, K\right\rangle .\right.
\end{aligned}
$$

Remark: An alternative way to prove the above theorem is the use of the inversion formula for the Laplace transform 


$$
f(t)=(2 \pi)^{-1} \int_{c-i \infty}^{\infty+\infty} f(s) \mathrm{e}^{s t} d s
$$

So, we may compute the user cost of capitulal as

$$
p_{K}(t)=(2 \pi)^{-1} \int_{c-i \infty s}^{c+i \infty}\left[\tilde{p}_{I}(s) / \tilde{h}(-s)\right] e^{s t} d s
$$

where

$$
p_{I}(s)=\int_{-\infty}^{\infty} p_{I}(t) \mathrm{e}^{-r t} d t=p_{I}(0)(s+r)^{-1}
$$

In the general case we suppose that the past investment flow

$$
I_{-}(t)= \begin{cases}l(t) & (t<0), \\ 0 & \text { (otherwise) }\end{cases}
$$

is not equal to zero everywhere. Then we have $I(t)-I_{-}(t)=0$ for all $t<0$. Hence, by the above theorem we have the equalities

$$
\left\langle p_{I}, I-I_{-}\right\rangle=\left\langle p_{R}, h *\left(I-I_{-}\right)\right\rangle, p_{K}=\tilde{h}(r)^{-1} p_{I} .
$$

Moreover, $p_{I}(t)=0$ for all $t<0$, implies $\left\langle p_{I}, I\right\rangle=\left\langle p_{I}, I-I_{-}\right\rangle$. Hence, we have

$$
\left\langle p_{I}, I\right\rangle=\left\langle p_{I}, I-I_{-}\right\rangle=\left\langle p_{K}, h *\left(I-I_{-}\right)\right\rangle=\left\langle p_{K}, K\right\rangle-\left\langle p_{K}, h_{*} I_{-}\right\rangle .
$$

The subtracted cost $\left\langle p_{K}, h * I_{-}\right\rangle$, due to past investments $I_{-}$, is a given constant, because $p_{K}, h$, and the past investments $I$ are known. Thus in the general case we may interpret the given solution $p_{K}$ as the marginal cost of capital.

\subsection{Critique on the User Cost of Capital in Empirical Moidels}

The theory in the previous section is of interest, because many economic models are based on the assumption that the discounted investment price is of the form

$$
p_{I}(t)=p_{I}(0) \mathrm{e}^{-r t} \quad(t \geq 0),
$$

where $r$ is a given discount rate In this special case we can easily derive the user cost of capital, given an arbitrary survival function, as is shown in the previous section. This means a freedom to choose a survival function in empirical economic models, which is not found in current literature. Nearly all investment survival functions one encounters in empirical economic models are of the exponential or rectangular forn. However, if we may believe the results in a publication of the Netherlands Central Bureau of Statistics (1986), a better form of the investment survival function can be derived from a gamma retirement, distribution.

The gamma probability distributions are of the form

$$
p(t)=\lambda^{\alpha} t^{\alpha-1} \mathrm{e}^{-\lambda t} / \Gamma(\alpha) \quad(t \geq 0)
$$

where

$$
\Gamma(\alpha)=\int_{0}^{\infty} t^{\alpha-1} \mathrm{e}^{-t} d t
$$

One has for $\alpha$ the relation $\alpha=(\mu / \sigma)^{2}$, where $\mu$ is the mean and $\sigma^{2}$ the variance of the gamma distribution. For $\alpha=1$, the corresponding survival function

$$
h(t)=1-\int_{0}^{t} p(\tau) d \tau \quad(t \geq 0)
$$

is exponential, while for $\alpha \rightarrow \infty$ - keeping $\mu$ constant - the limiting 
corresponding survival function is rectangular. The Netherlands Central Bureati of Statistics (1986) found values of o between 2 and 3.5 , when the retirement of different capital assets was investigated.

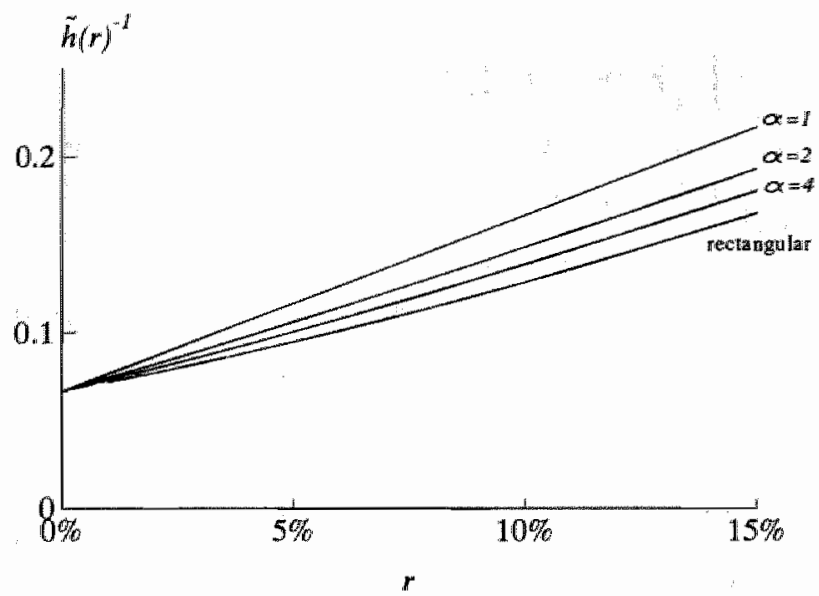

Figure 18.1 User Cost Capital

The question what the error may be, when we use the exponential or rectangular survival function instead of a more realistic survival function, is of interest. From Theorem 18.1 we derive for gamma investment retirement distributions with an exponential discounted investment price the equality

$$
p_{K} / p_{I}=\tilde{h}(r)^{-1}=r\left[1-(1+r / \lambda)^{-\alpha}\right]^{-1} \text {. }
$$

Figure 18.1 compares the above expression in case of the exponential $(\alpha=1)$ and the rectangular $(\alpha \rightarrow \infty)$ function to the case in which a more realistic form $(\alpha=2,4)$ is chosen. The mean investment lifetime $\mu$ is in all cases equal to 15 years. It appears that the exponential survival function overestimates the value of the user cost of capital with an error of approximately $10 \%$, while the rectangular function underestimates the user cost of capital with an ertor of the same magnitude. As is evident from this example, it is not only the mean investment lifetime that determines the user cost of capital but anso the shape of the retirement curve.

\subsection{Other Survival Functions}

The previous section criticizes the use of survival functions in empirical models and specifies a surviwal function, which might be a better choice. One may reply again that it is doubtcul whether this specific form of the survivall function is a good choice. In this section I will argue that it is not the specific parametric form of the survival function that is the most important, but the statistical properties of the corresponding retirement distribution. To make a good choice of a survival function, it is usually sufficient to specify the mean and variance of the corresponding retirement distribution. Because this specification is possible for the family of gammat distributions, we may use this family to compute the user cost of capital. 
Let us consider the case in which investment cost is of the form

$$
p_{I}(t)=p_{I}(0) e^{-n t} \quad(t \geq 0)
$$

Then the user cost of capital is equal to $p_{K}=p_{\Gamma} / \tilde{h}(r)$. where ${ }^{n}$ is the Laplace ransform of the survival function $h$. The following theorem yields an appoximation of $\tilde{h}(r)$ in case $r$ is small.

Theorem 18.2: For survival functions h one has

$$
h(r) \approx r^{-1}\left[1-e^{-r / p}\left(1+\sigma^{2} r^{2} / 2\right)\right] \quad(r+0),
$$

where $\mu$ is the mean and $\sigma^{2}$ is the variance of the corresponding rnetirement distribution $p$.

Proof: One can expand the Laplace transform of a probability distribution $p$ around $t=\mu$ as follows.

where

$$
\tilde{p}(s)=\mathrm{e}^{-s \mu}\left[1+\sum_{n=2}^{\infty} \mu_{r_{i}}(-s)^{n} / n !\right],
$$

$$
\mu_{n}=\int_{-\infty+\infty}^{\infty} p(t)(t-\mu)^{n} d t
$$

is the $n$-th central moment of the probability distribution $p$. This follows from

$$
\begin{aligned}
\tilde{p}(s) & =\int_{-\infty}^{\infty} p(t) \mathrm{e}^{-s t} d t=\mathrm{e}^{-s \mu} \int_{-\infty}^{\infty} p(t) \mathrm{e}^{-s(1-\mu t)} d t \\
& =e^{-s \mu} \int_{-\infty}^{\infty} p(t) \sum_{n=1}^{\infty}[-s(t-\mu)]^{n} / n ! d t \\
& =e^{-s \mu}\left[1+\sum_{n=2}^{\infty} \mu_{n 2}(-s)^{n} / n !\right] .
\end{aligned}
$$

For $|s|$ small, we may approximate $\tilde{p}$ by the first two terms of this expansion, and we obtain

$$
\tilde{p}(s) \approx e^{-s \mu}\left(1+\sigma^{2} s^{2} / 2\right)
$$

where $\sigma^{2}=\mu_{2}$ is the variance of the retirement distribution. Thus the Laplace transform of the survival function can be approximated by

$$
\tilde{h}(s) \approx s^{-1}\left[1-e^{-s \mu}\left(1+\sigma^{2} s^{2} / 2\right)\right] \quad(|s|+0)
$$

which depends only on the mean $\mu$ and variance $\sigma^{2}$ of the retirement distribution. Note that in case of symmetric retirement distribution one has

$$
\mu_{r 1}=0 \quad(n \text { odd } \text { and } n>1) \text {, }
$$

and then the given approximation of $\tilde{p}(s)$ is in fact a third order approximation.

Figure 18.2 compares the approximation of the user cost of capital, given in the above theorem, to the results in Figure 18.1. There are three examples of approximation given, which appear just above the user cost hat is approximated. The fourth approximation of the rectangular survival function

$$
\tilde{h}(s)=s^{-1}\left(1-\mathrm{e}^{-s: p}\right) \quad(s \in \mathbb{C})
$$

is exact, because in that case all higher central moments are equal to zero. From Figure 18.2 it appears that the approximation works well in case of gamma distributions with parameter $\alpha$ larger than one. For $\alpha=1$ the approximation does not work very well, because the approximation is based on an expansion around the mean, assuming that the mean is in the neighibourhood of the mode, and the mean and the mode of the exponential retirement 
distribution do not connclde at all. The approximation works good in case a is larger than one, because the distribution is more symmetric. For $\alpha$ in the area where the surviwal function has a more realistic form the fit is very good.

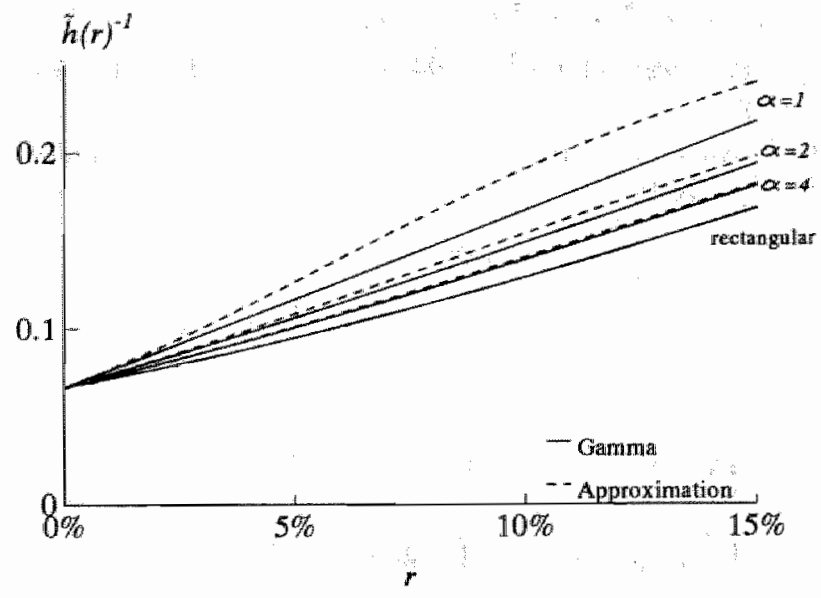

Figure 18.2 Approximation User Cost

We can give another simple approximation when $r$ is high. Then the user cost of capital will mainly depend on $r$. When $p$ is a probability distribution then we may show that $\tilde{p}(r)$ approaches zero, when $r$ increases, and we have then

$$
p_{K} / p_{I}=\tilde{h}(r)^{-1}=r[1-\tilde{p}(r)]^{-1} \approx r \text {. }
$$

We may summarize the above results, concerning the determination of the user cost of capital, as follows. When the discounted investment price is of the exponential form

$$
p_{I}(t)=p_{I}(0) \mathrm{e}^{-r t} \quad(t \geq 0)
$$

and the investment retirement distribution is maximal ir the neighbourhood of the mean investment lifetime, then the user cost of capital is approximately determined by the mean and variance of the retirement distribution.

\subsection{Summary}

A nice result is the simple solution of the user cost of capital $p_{K}$ in case capital $K$ can be described as $K=h * I$ and the discounted investment price

$$
p_{x}(t)=p_{l}(0) \mathrm{e}^{-r t}
$$

is of the exponential form. In this special case the user cost of capital $p_{K}$ is equal to $p_{r} / \tilde{h}(r)$.

Furthermore, it shown that the parametric specification of the investment survival function th is relatively unimportant in case the discount rate $T$ is low. Then it are mainly the statistical properties of the corresponding retirement distribution, like mean and variance of the investment lifetime, which determine the user cost of capital. It is shown that the shape of the retirement curve has a substantial influence on the user cost of capital 
solution. Hence, it is not sufficient to consider only the mean lifetime of capital assets, but one should also consider the variance of the lifetime.

In this chapter the user cost of capital for a given exponentiall survival runction is compared with the user cost of capital derived from a gamma retirnement distribution, that has a more realistic shape. It appears that when the discounted investment price is exponentially decreasing, the exponential survival function overestimates and the rectangular survival function underestimates the user cost of capital. 


\section{GENERATION OF CAPITAL STOCKS}

\subsection{Introduction}

With the perpetual inventory model the capital stock $K$ is described as

$$
K=h * I=\int_{-\infty}^{\infty} h(t-\tau) I(\tau) d \tau
$$

where $I$ is investment flow and $h$ the survival function of investments. A drawback to the generation of capital stocks, using the above equation, is the evaluation of the integral for every $t$. Another drawback is the need for investment data, covering a long period in the past. These drawbacks are not present when the capital stock is generated by a linear system of the form

$$
\dot{x}=A x+b I, K=c x,
$$

where $A$ is a real matrix, $b$ a real vector and $c$ a transposed real vector. In that case it is sufficient to know the initial value $x(0)$, to start the simulation at $t=0$. Such a linear system generates the same results as a perpetual inventory model with survival function

$$
h(t)=\operatorname{ces}^{A t} b \quad(t \in \mathbb{R})
$$

where

$$
\mathrm{e}^{A t} \equiv \sum_{n=0}^{\infty}(A t)^{n} / n ! \quad(t \in \mathbb{R})
$$

e.g. see Kailath $(1980$, page 70$)$. The triple $(A, \delta, c)$ is said to be a state-space realization for survival function $k$.

So, we may simplify the generation of capital stocks, using a state-space realization for the investment survival function. However, not every survival function has a simple state-space realization. This urges a family of survival functions with the following desired properties:

1. Every member has a simple state-space realization.

2. The shape of the members approximates the empirical shape of the investment survival function.

3. Every given positive mean and variance of the corresponding retirement distribution has a representative member in the family.

Such a family is useful for generating capital stocks, because retirement distributions with the same mean and variance tend to generate similar capltal stocks. As 1 will point out below, the latter is especially the case for investment flows, which can be described as the superposition of a slowly varying function and last fluctuations. Of course, the generation of capital stocks is obviously improved when the actual shape of the retirement distribution is approximated.

That the statistical properties of the retirement distribution are important follows from the results in Chapter 18. There it was shown that the Laplace transform of the corresponding survival function $h$ may be approximated by

$$
\tilde{h}(s) \approx s^{-1}\left[1-e^{-s / \mu}\left(1+\sigma^{2} s^{2} / 2\right)\right] \quad(s \in \mathbb{C}:|s| * 0) .
$$

This means that retirement distributions with the same mean and variance tend to generate similar capital stocks when the variation in the investment flow is small. Also this is the case for fast fluctuations in the investment flow, because one has 


$$
\tilde{h}(s) \approx s^{-1} \quad(s \in \mathbb{C}:|s| \rightarrow \infty) .
$$

This follows from $\tilde{h}(s)=s^{-1}[1-\tilde{p}(s)]$, where $p$ is the investment retirement

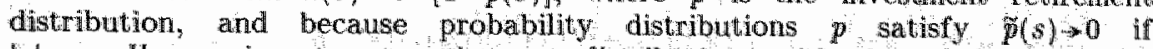
$|s| \rightarrow \infty$. Hence, investment retrement distributions with the same mean and variance tend to generate simlar capital stocks in case of a superposition of slow and fast investment fuctuations. Extension of the above results to a superposition is allowed, since the capital stocks are generated by a linear system.

\subsection{Contagious Erlang Distributions}

In Paccoud (1983) several families of probability distributions are given, which are used as retirement distribution to generate capital stocks. One of these families is the family of gamma distributions. The Statisches Bundesamt in Germany generates capital stocks using gamma retirement distributions. Also in Berends-Ballast (1987), where several parametric families of distributions are inwestigated, the best fit to empirical data was achieved by using gamma distributions..

However, not every gamma distribution has a simple state-space realization. The Laplace transform of a gamma distribution is given by

$$
\tilde{p}(s)=(1+s / \lambda)^{-\infty} \quad(s \in \mathbb{C}),
$$

and the Laplace transform of the corresponding survival function is

$$
\tilde{h}(s)=s^{-1}\left[1-(1+s / \lambda)^{-\alpha}\right] \quad(s \in \mathbb{C}) .
$$

For the existence of a state-space realization, given a survival function $h$, it is necessary that one can write $\tilde{h}(s)=a(s) / b(s)$, where $a(s)$ and $b(s)$ are polynomes, e.g. see Kailath $(1980$, page $66-69)$. So gamma distributions, such that $\alpha$ is integer, may be used to generate capital stocks with a state-space realization. For $\alpha=1$ one obtains the exponential survival function, which is widely used. Further, the case $\alpha=2$ is used in Almon et al. (1974) and Hahn and Schmoramz (1984). Finally, Stahmer (1983) propagates the use of the general case, where $\alpha$ is integer.

Definition: The subfamily of gamma distributions of the form

$$
p_{k}(\lambda ; t)=1(t) \lambda^{k} t^{k-1} \mathrm{e}^{-\lambda t} /(k-1) ! \quad(k \in \mathbb{N}, \lambda>0, t \in \mathbb{R})
$$

are called Erlang distributions.

The Laplace transform of such an Erlang distribution is

$$
\tilde{p}_{k}(\lambda ; s)=(1+s / \lambda)^{-k} \quad(s \in C) \text {. }
$$

The family of Erlang distributions does not contain a representative member tor every positive mean and variance. Therefore $I$ define a family of contagious Erlang distributions.

Definition: A contagious Eriang distribution is defined as a weighted mean of two Erlang distributions

$$
p(t)=\beta p_{k-1}(\lambda ; t)+(1-\beta) p_{k}(\lambda ; t) \quad(0 \leq \beta<1, \lambda>0, k \in \mathbb{N}, t \in \mathbb{R}) .
$$

The mean and variance of contagious Erlang distributions are as follows.

Theorem 19.1: Suppose

$$
p(t)=\beta p_{k-1}(\lambda ; t)+(1-\beta) p_{k}(\lambda ; t) \quad(t \in \mathbb{R}),
$$

is a comtagious Erlang distribution. Then

$$
\mu=E[t]=(k-\beta) / \lambda, \sigma^{2}=E\left[(t-\mu)^{2}\right]=\left(k-\beta^{2}\right) / \lambda^{2} \text {. }
$$


Proof: We obtain

$$
\begin{aligned}
& E[t]=\int t\left[\beta p_{k-1}(t)+(1-\beta) p_{k}(t)\right] d t=\beta(k-1) / \lambda+(1-\beta) k / \lambda=(k-\beta) / \lambda \\
& E\left[(t-\mu)^{2}\right]=E\left[t^{2}\right]-\mu^{2}=\int t^{2}\left[\beta p_{k-1}(t)+(1-\beta) p_{k}(t)\right] d t-\mu^{2} \\
&=\beta\left[(k-1)^{2} / \lambda^{2}+(k-1) / \lambda^{2}\right]+(1-\beta)\left[k^{2} / \lambda^{2}+k / \lambda^{2}\right]-(k-\beta)^{2} / \lambda^{2} \\
&=\left(k-\beta^{2}\right) / \lambda^{2} .
\end{aligned}
$$

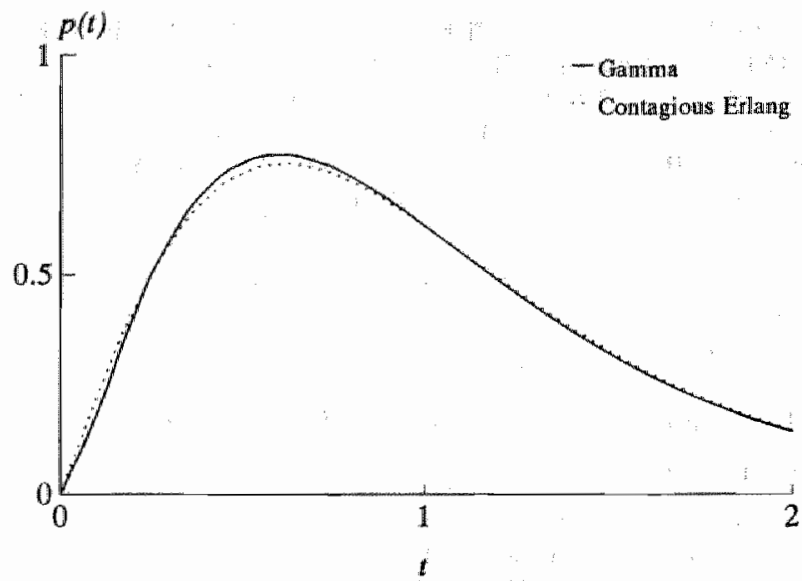

Figure 19.1 Gamma Approximation

Now I show that for every given positive mean and variance there is a unique contagious Erlang distribution.

Theorem 19.2: Suppose $\mu>0$ and $\sigma^{2}>0$. Then there exists a unique contagious Erlang distribution with mean $\mu$ and variance $\sigma^{2}$, for which the parameters $k$, $\lambda$ and $\beta$ are determined by

$$
k-1<\mu^{2} / \sigma^{2} \leq k, \lambda=\left\{\mu+\left[\left(\mu^{2}+\sigma^{2}\right) / k-\sigma^{2}\right]^{1 / 2}\right\} /\left\{\left(\mu^{2}+\sigma^{2}\right) / k\right\}, \beta=k-\lambda \mu,
$$

where $k \in \mathbb{N}$.

Proof: From $\mu=(k-\beta) / \lambda$ lol.lows $\beta=k-\lambda \mu$. Hence, one has

$$
\sigma^{2}=\left(k-\beta^{2}\right) / \lambda^{2}=\left[k-(k-\lambda \mu)^{2}\right] / \lambda^{2}
$$

which can be written as

$$
\left(\mu^{2}+\sigma^{2}\right) \lambda^{2}-2 k \mu \lambda+k^{2}-k=0 .
$$

Since $\lambda$ is positive, this means

$$
\begin{aligned}
\lambda & =\left\{2 k \mu+\left[(2 k \mu)^{2}-4\left(\mu^{2}+\sigma^{2}\right)\left(k^{2}-k\right)\right]^{1 / 2}\right\} / 2\left(\mu^{2}+\sigma^{2}\right) \\
& =\left\{\mu+\left[\left(\mu^{2}+\sigma^{2}\right) / k-\sigma^{2}\right]^{1 / 2}\right\} /\left\{\left(\mu^{2}+\sigma^{2}\right) / k\right\} .
\end{aligned}
$$

This means that one should have $\left(\mu^{2}+\sigma^{2}\right) / k-\sigma^{2} \geq 0$ and thus $k-1 \leq \mu^{2} / \sigma^{2}$. lurthermore, since $0 \leq \beta \leq 1$, one has $\beta^{2} \leq \beta$ and thus 


$$
\mu^{2} / \sigma^{2}=(k-\beta)^{2} /\left(k-\beta^{2}\right) \leq k-\beta \leq k
$$

For $\alpha \geq 2$, the contagious Erlang distributions appear to be a good approximation of gamma distributions, which have the same mean and variance. An example of such an approximation is given in Figure 19.1, where a gamma distribution is considered with $\alpha=2.5$.

\subsection{State-Space Realization for a Contagious Erlang Distribution}

In the above section 1 described the use of contagious Erlang distributions. It is shown that, given the mean and variance of the retirement distribution of capital, there exists a corresponding contagious Erlang distribution of the form

$$
p(t)=\beta p_{k-1}(\lambda ; t)+(1-\beta) p_{k}(\lambda ; t): \quad(t \in \mathbb{R}) .
$$

In this section I derive the form of the corresponding state-space realization, which generates the capital stock with the equations

$$
\dot{x}=A x+b I, K=c x \text {. }
$$

This realization is

$$
A=\left[\begin{array}{rrrrr}
-\lambda & 0 & . & 0 \\
\lambda & -\lambda & & . \\
0 & \lambda & & \cdot \\
\cdot & \cdots & . \\
. & . & . & 0 \\
0 & \cdots & 0 & \lambda & -\lambda
\end{array}\right], \quad b=\left[\begin{array}{l}
1 \\
\cdot \\
\cdot \\
\cdot \\
\cdot \\
1
\end{array}\right], \quad c=\left[\begin{array}{lllll}
0 & \cdots & 0 & \beta & 1-\beta
\end{array}\right]
$$

where $A$ is a $k \times k$ matrix.

The form of the survival function of an Erlang retirement distribution is shown in the following theorem.

Theorem 19.3: Suppose $\lambda>0$ and $p_{n}(\lambda ; t), n=1, \ldots, k$, is a series of Erlang distributions. Then

$$
1(\cdot) *\left(\delta(\cdot)-p_{k}\right)=\lambda^{-1} \sum_{n=1}^{k} p_{n}
$$

Proof: One can give a direct proof by using partial integration. The following proof with Laplace transforms is however easier. One has

$$
\begin{aligned}
\mathcal{L}\left[1(\cdot) *\left(\delta(\cdot)-p_{k}\right)\right] & =s^{-1}\left(1-\tilde{p}_{k}\right)=s^{-1}\left[1-(1+s / \lambda)^{-k}\right] \\
& =s^{-1}[(1+s / \lambda)-1] \sum_{n=1}^{k}(1+s / \lambda)^{-n}=\lambda^{-1} \sum_{n=1}^{k}(1+s / \lambda)^{-n} \\
& =\lambda^{-1} \sum_{n=1}^{k} \tilde{p}_{n^{*}}
\end{aligned}
$$

When the above theorem is combined with the following theorem then the given state-space realization can be derived.

Theorem 10.4: Suppose $\lambda>0$ and

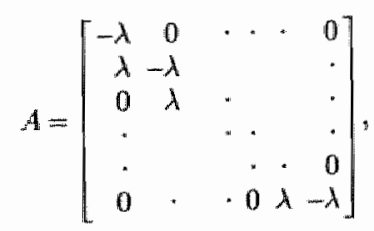

where $A$ is a kxk matrix. Then 


$$
\mathbb{e}^{A t}=\lambda^{-1}\left[\begin{array}{cccc}
p_{1}(\lambda, t) & 0 & * & 0 \\
p_{2}(\lambda, t) & p_{1}(\lambda, t) & \\
\cdot & & & \\
p_{k}(\lambda, t) & \cdot & & 0 \\
p_{1}(\lambda, t)
\end{array}\right]
$$

Proof: We may wite $A=\lambda(L-1)$, where 1 is the unity matrix and $L$ satisfies $L^{n}=0$ iff $n \geq k$. Because $L$ commutes with 1 , i.e. $L 1=1 L$, we have

$$
e^{A t}=e^{(L-1) \lambda t}=\mathrm{e}^{L \lambda t} \mathrm{e}^{-\lambda t}
$$

thus we obtain

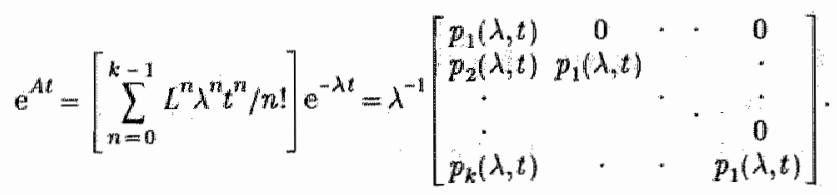

The above theorems prove that the given state-space realization corresponds to a contagious Erlang distribution.

Theorem 19.5: Suppose

$$
p(t)=\beta p_{k-1}(\lambda ; t)+(1-\beta) p_{k}(\lambda ; t) \quad(t \in \mathbb{R})
$$

is a contagious Erlang distribution and put

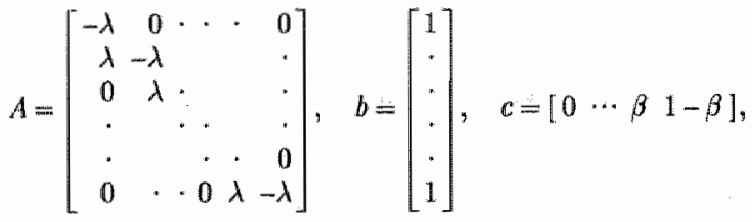

where $A$ is a $k \times k$ matrix. Then $(A, b, c)$ is a state-space realization for survival function

$$
h=1(\cdot) *(\delta(\cdot)-p) \text {. }
$$

Proof: Apply Theorem 19.3 and Theorem 19.4 to

$$
h(t)=\mathrm{ce}^{A t} b \quad(t \in \mathbb{R})
$$

\subsection{Initialization of the State-Space Realization}

Consider a state-space realization $(A, b, c)$ as described above. To start the simulation of

$$
\dot{x}=A x+b I, K=c x,
$$

we need to initialize $x(0)$. I will give two ways to approximate $x(0)$. For the first approximation $\mathbb{I}$ consider a constant investment flow $I(t)=I(0)$, for all $t \leq 0$, in the past. For the second approximation I consider a fixed growth rate $s \in \mathbb{R}$ of the iravestment flow

$$
I(t)=I(0) e^{\text {at }} \quad(t \leq 0),
$$

in the past, and a known current retirement flow of investments $R(0)$.

Using Theorem 19.3 and 19.4 , and the equality

$$
x=\left[\mathrm{e}^{A t} b\right] * I \text {, }
$$

we obtain $x_{n}=h_{n} * 1$, for $n=1, \ldots, k$, where $h_{n}$ is the survival function corresponding to an Erlang retirement distribution $p_{n}(\lambda ; t)$. Hence, because 
the mean lifetime for $h_{n}$ is equal to $n / \lambda$, we obtain in case of a constiant investment flow at $t \leq 0$ the approximation

$$
x_{n}(0)=(n / \lambda) I(0) \quad(n=1, \ldots, k),
$$

A more sophisticated approximation is derived by assuming the investment flow of the form

$$
r(t)=I(0) \mathrm{e}^{\text {si }} \quad(t \leq 0) \text {, }
$$

with a fixed rate of growth $s \in \mathbb{R}$. Then one has

$$
x_{n}(0)=\tilde{h}_{n}(s) I(0)=s^{-1}\left[1-(1+s / \lambda)^{-n}\right] I(0) \quad(n=1, \ldots, \ldots, k)
$$

Now, to determine $x_{n}(0)$, we need to know the rate of growth s. The following method is useful when the retirement flow of unvestments $R(0)$ is known.

Since the rate of growth $s$ is constant, we have the equality $R(0)=\tilde{p}(s) /(0)$, where $p$ is the retirement distribution. Because the contagious Enlang distribution approximates the gamma distribution with the same mean $\mu$ and wariance $\sigma^{2}$, we have

$$
\tilde{p}(s) \approx(1+s / \gamma)^{-\alpha} \quad\left(\alpha=\mu^{2} / \sigma^{2}, \gamma=\mu / \sigma^{2}\right) .
$$

By inverting this equation, we can approximate the rate of growth

$$
s \approx\left[(R(0) / I(0))^{-1 / \alpha}-1\right] \gamma \quad\left(\alpha=\mu^{2} / \sigma^{2}, \gamma=\mu / \sigma^{2}\right) .
$$

\subsection{The Discrete-Time Model}

The theory in this chapter describes a continuous-time model. The empirical data, however, are discrete. The continuous-ime model simplifies the mathematics, but to apply this model to empirical data it has to be reformulated into a discrete-time model. To derive the discrete-time model we replace $\dot{x}=A x+b I$ by

$$
[x(t+\Delta t)-x(t)] / \Delta t=A x(t)+b I(t)
$$

Thus the continuous model reformulated as a discrete-time model is

$$
x^{t+1}=(I+\Delta t A) x^{t}+\Delta t b I^{t}, K^{t}=c x^{t} .
$$

Note that the length of simulation period $\Delta t$ may not be too long. Otherwise the discrete-time model will not approximate the results of the continuous model. 


\section{SUMMARY}

The subject "Nonparametric Consumex and Producer Analysis" is related to the nomparametric statistical theory. This statistical theory is not based on a parametric specified probability distribution, but postulates instead that the probability distribution is member of a nomparametric family of probability functions. Such a family is defined by means of symmetry properties of the probability distribution.

The analogy of this approach in economic theory is using a nomparametric specification of utility and production functions. One may postulate that observations, concerning consumer and producer behaviour, is generated by a function that satisfies such a nonparametric specification. By developing tests to check whether such a hypothesis is satisfied, one may test the significance of assumptions concerning economical behaviour and the form of the utility or production function. These nonparametric tests may be of help in case one chooses a parametric specification of economic behaviour.

This thesis describes many of these monparametric tests. They are concerned with the economic behaviour of utility maximization, profit maximization and cost minimization, in relation to which the following nonparanetric specifications are considered: linear homogeneity, homotheticity, separability, and technical progress. The nomparametric tests are applied to Dutch consumer and industry data, showing the possibilities which the theory offers. Moreover, as an experiment the nonparametric tests of consumer behaviour are applied to aggregate random data. The random data were aggregated in the same way as the Dutch consumer data were aggregated by the Netherlands Central Bureau of Statistics.

In general the nonparametric tests do not indicate which specification has to be used. However, they show whether specifications are incompatible with the data. So they indicate which specifications one should certainly not use. A clear example are the nonparametric test results in this book concerning the Dutch industry data. These results indicate that profit maximization, not including any assumption of technical progress, cannot be used as a behaviour model for the Dutch industry data. However, one may use a profit maximization model that includes technical progress. Another possibility, concening these data, which does not assume technical progress, is the choice of a cost minimization model.

The results of the nomparametric tests show that one has to be careful in drawing conclusions from aggregate data. For it appeared that aggregate random data satisfied the hypothesis of consumer demand. This is caused by using an obvious rule concerning consumer denand: price times quantity equals expenditures. But prices and corresponding quantities tend to move in opposite direction through this rule, exactly what one expects of consumer behaviour. The belief in consumer behaviour acts in this case as a self-fulfilling prophecy.

In practice nonparametric tests concerning efficient economic behaviour nearly always reject the postulated hypothesis. Two ways are described to derive a measure of violation of the hypotheses. First, one may delete as few observations as possible from the data, to such an extent that the remaining observations do satisfy the hypothesis. Secondly, one may weaken the hypothesis by considering inefficient behaviour. In that case the maximal 
efficiency, for which the data still satisfies the hypothesis, indicates how seriously the hypothesis is violated.

Although the emphasis in this book is put on the development of nomparametric tests, concerning consumer and producer behaviour, ample attention is pard to the problem of the derivation of prices and quantities of capital stocks. It is shown that the conventional exponential decay of capital assumption can be improved for both the generation of capital stocks and the user cost of capital. Assuming that the retirement of capital may be described by a gamma distribution, a simple method is presented that approximates the capital stocks by using a linear dynamic model. Furthermore, it is described how one may derive the user cost of capital for an arbitrary given investment retirement distribution.

\section{Generalizations of the Nonparametric Theory}

The nomparametric tests in this book are presented in the form of theorems. These theorems are proved by constructing a suitable utility or production function for the given data. Hence, if the data satisfy certain properties, like concave budget constraints, this implies certain properties concenning the constructed function. This means that many variations are possible on the theorems, in which properties of the constructed function are implied by assumptions concerning the data. Another generalization, which can often be achieved without problems, is the generalization from the single-output production function to the case where multiple ontputs are allowed.

The differentiability of utility and production functions is not considered. An excellent short article of Chiappori and Rochet (1987) contains a proof that derives an infinitely differentiable utility function as the convolution of two functions. When one is interested in the existence of a differentiable utility or production function, their method can be applied to many results in this book.

Now that we have an overview of the results, we may search for comnections between de several nonparametric theorems with the aim to include them in a more general theory. Such a theory can answer the question for which specificiations one may derive a nonparametric theorem. At the beginning of this research this was mainly a matter of trial and error. Later it became apparent why certain specifications are a problem and others not. The global issue in such a theory is the symmetry of the optimization problem. For example, linearly homogeneous cost minimization, for which the Lagrangian shows symmetry, did not give problems. This in contrast to the results in case of weak separability, for which the Lagrangian does not display such an obvious symmetry.

\section{Applications of the Nonparametric Theory}

What are the applications of the nonparametric theory? First, it can be used to get an overview of the properties of a data set. This may save time when searching for a parametric specification of the utility or production function. The nomparametric tests may serve in that case as a guide, showing the possibilities that one has. Next, the nonparametric theory can be a tool in solving theoretical problems. For example, problems which consider the existence of utility functions or production functions, concerning a given demand relation.

One application of the nonparametric approach, that is especially useful in a producer context, is the forecast of economic behaviour. This was not discussed in this book, but the tools are presented in the form of bounds on the production function, and bounds on technical efficiency and technical 
progress. These bounds can be seen as conserved properties that are due to the symmetry of the optimzation model, which generates the data. In physics one finds andogical examples of conserved properties, like energy and momentum, which also proceed from the fact that the Lagrangian is symmetric. The nomparametric approach, with symmetry assumptions as a starting-point, is a suandard tool in advanced physics. For example, it has been used to predict the existence of new elementary particles. We might expect that such a nomparametric approach will also be of value in economics. 


\section{APPENDIX}

\section{A BINARY RELATIONS}

\section{A.1 Binary Relations}

Let $X$ be an arbitrary set of elements. A binary relation $R$ on the set $X$ is a subset of the Cartesian product $X \times X$, that is the set of ordered pairs $(x, y)$ such that $x$ and $y$ are in $X$. If the ordered pair $(x, y)$ belongs to $R$, one denotes indifferently $(x, y) \in R$ or $x R y$.

Given a birary relation $R$ on $X$, the complement $R^{c}$ is defined as the binary relation on $X$ for which one has

$$
x R y \Leftrightarrow y R^{c} x \quad(x, y \in X)
$$

The transitive closure $R^{*}$ is defined as the binary relation on $X$ for which one has $x R^{*} y$ if there exists a finite sequence $x=x^{1}, x^{2}, \ldots, x^{n}=y$ in $X$ such. that

$$
x^{m} R x^{m+1} \quad(m=1, \ldots, n-1) .
$$

A quilck way to compute this closure, given by Warshall (1962), may be found in Appendix C.5.

\section{A.2 Basic Properties of Binary Relations}

Let $R$ be a binary relation on the set $X$, and let $x, y$ and $z$ be arbitiary elements in $X$. Then $R$ is:

- reflexive

- irreflexive

- symmetric

- antisymmetric

- asymmetric

- complete

- strongly complete

- transitive

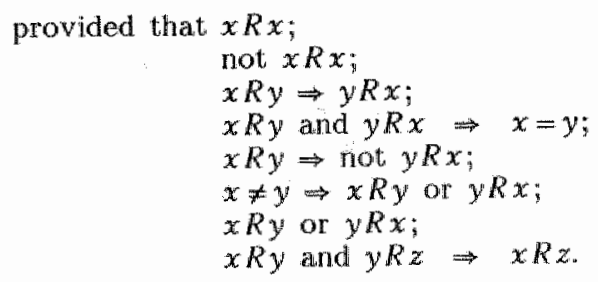

\section{A.3 Particular Binary Relations}

A transitive binary relation $R$ on the set $X$ is a:

- prearder or quasi order if $R$ is reflexive;

- total preorder or weak order if $R$ is strongly complete;

- partial order if $R$ is reflexive and antisymmetric;

- strict partial order if $R$ is asymmetric;

- total order if $R$ is antisymmetric and strongly complete;

- strict total order or linear order if $R$ is asymmetric and complete;

- equivalence relation if $R$ is reflexive and symmetric.

For preorders Herstein and Milnor's (1953) notation is used. In that case a preorder is denoted as $\succsim$, using the notations: 


$$
\begin{aligned}
& x \sim y \Leftrightarrow x \succsim y \text { and } y \succsim x, \\
& x>y \Leftrightarrow x \succsim y \text { and not } y \succeq x, \\
& x \lesssim y \Leftrightarrow y \succsim x, \\
& x<y \Leftrightarrow y \succ x .
\end{aligned}
$$

For any preorder it holds that $\succ$ is a strict partial order and $\sim$ is an equivalence relation. We refer in the text to a preorder $\succsim$ as $>$ for typographical convenience.

\section{A.4 Extensions of Orders}

Szpilrajn"s theorem, which states that any partial order can be extended to a total order is important.

Theorem A.1: Let $\succ$, be a partial order on a set $X$. Then there exists a total order $\succ^{\prime}$, m $^{\prime}$ on $X$ such that $\succsim \subset \beth^{\prime}$ and $>c \succ^{*}$.

Proof: See Sepilrajn (1930).

Similarly one may extend a preorder to a weak order. A proof of this theorem, using a graph theory result, may be found in Roubens and Vincke (1985, page 49). I will give an alternative proof that is based on Szpilrajn's theorem.

Theorem A.2: Let $\succ \sim$ be prearder on a set $X$. Then there exists a weak order $\chi^{\prime \prime} \sim$ " on $X$ such that $\succsim c \succsim$ ' and $\succ c>$.

Proor: The projection $\Pi: X \rightarrow X / \sim$ induces a preorder $\zeta_{2} \approx$ on $X / \sim$, defined by

$$
\text { IIx } \sum \| y \text { if } x \succsim y \quad(x, y \in X) \text {. }
$$

Since $\zeta, \sim$ is a preorder, it is easy to show that $\zeta, \approx$ is a partial order on $X / \sim$. Hence, by Szpilrajn's theorem, $\Sigma_{,} \approx$ can be extended to a total order $\zeta^{\prime}, \approx^{\prime}$ on $X / \sim$, such that $\bar{\zeta} \Sigma^{\prime}$ and $\zeta \subset \zeta^{\prime}$. Now, define the preorder $\succ^{\prime}, \sim$ on $X$ as

$$
x \succsim^{\prime} y \text { if } \Pi x \beth^{\prime} \Pi y \quad(x, y \in X)
$$

which is obviously a weak order. Furthermore, since

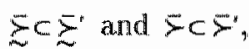

it is straightforward to show that one has $\succsim \subset \succsim^{\prime}$ and $\supset \subset \succ^{\prime}$.

In general a preorder cannot be extended to a partial order, but the following weaker theorem is avalable.

Theorem A.3: Let $\infty, \infty$ be prearder on a set $X$. Then there exists a partakn arder $\succ$; $\sim$ on $X$ such that $\succ \subset \succ$.

Proof: Define the partial order $\succ^{\prime}, \sim^{\prime}$ on $X$ by

$$
x \underset{\sim}{>} y \text { if }(x>y \text { or } x=y) \quad(x, y \in X) \text {. }
$$

To show that $>\subset>$, suppose $x>y$ for arbitrary $x, y \in X$. Then one has

$$
x x^{\prime \prime} y
$$

by definition, and $x \neq y$. Hence $x \succ^{\prime} y$, because the partial order $>^{\prime}, \sim^{\prime \prime}$ is antisymmetric. Thus

$$
x>y \Rightarrow x>^{\prime} y \quad(x, y \in X),
$$

which means that we have $\succ c \succ^{*}$. 


\section{A.5 Extensions of Binary Relations}

A nonparametric analysis of consumer data may reveal a part of consumers preferences. In general the revealed part of consumers preferences can be described by two binary relations $R$ and $P$ on a set $X$ with the following interpretation for consumers preferences:

$$
\begin{aligned}
& x R y \Rightarrow y \text { is not preferred to } x, \\
& x P y \Rightarrow x \text { is preferred to } y .
\end{aligned}
$$

A problem now is the question whether the relations $R$ and $P$ can be extended to a preorder, representing the preferences of a rational consumer. In a mathematical formulation this problem is the following:

Given two binary relations $R$ and $P$ on $X$, is there a preorder $>, \sim$ on $X$ such that $R \subset \succeq$ and $P \subset \succ$ ?

Necessary and sufficient conditions for the existence of such a preorder are stated in the following theorem.

Theorem A.4: Let $R$ and $P$ be arbitrary binary relations on a set $X$. Then the following conditions are equivalent:
(i) There existis a preorder $\succ$, on $X$ such that

$$
R \subset \succsim, P \subset>\text {. }
$$

(ii) There exists a weak order $\succ$, on $X$, satisfying the above condition.

(iii) One has $(R \cup P)^{*} \cap P^{c}=\varnothing$.

Proof (i) $\Rightarrow$ (ii): Follows from Theorem A.2.

(ii) $\Rightarrow$ (i): Obvious, because any weak order is a preorder.

(ii) $\Rightarrow$ (iii): For any preorder $\succ, \sim$ one has

$$
(R \cup P)^{*} \cap P^{c} \subset(\succsim \cup \succ)^{*} \cap \succ^{c}=\beth^{*} n<=\succsim n \prec=\emptyset \quad(R \subset \succsim, P \subset>) .
$$

(iii) $\Rightarrow(i)$ : Define the binary relation $\succsim$ on $X$ by

$$
x \succsim y \Leftrightarrow(x, y) \in(R \cup P)^{*} \text { or } x=y \quad(x, y \in X)
$$

This is clearly a preorder, such that

$$
R \subset \succsim \text { and } P \subset \succsim \text {. }
$$

To show that $P \subset \succ$, suppose $x P y$ for arbitrary $x, y \in X$. Then $x \succsim y$, because $P \subset \succsim$. Furthermore, (iii) implies that one has

$$
x P y \Rightarrow(y, x) \notin(R \cup P)^{*} \Rightarrow \text { not } y P x \Rightarrow x \neq y \text {. }
$$

Thus $(y, x) \notin(R \cup P)^{*}$ and $x \neq y$, which means that one has not $y \succsim x$. Hence we obtain $x \succ y$ and not $y \succeq x$, so that $x \succ y$. Thus $P<\succ$.

In empirical applications of the revealed preference axiom to consumer data. one has often $R=\emptyset$ in the problem given in the begimning of this section. In that case all relevant information is contained in the binary relation $P$, and one may use the following theorem.

Theorem A.5: Let $P$ be a binary relation on a set $X$. Then the following conditions are equivalent:

(i) There exists a weak order $\succ$, on $X$ such that $P \subset \succ$.

(ii) There exists a prearder $\succ, \sim$ on $X$ such that $P \subset \succ$.

(iii) There exists a partial order $\succ \sim$ on $X$ such that $P \subset \succ$.

(iv) There exists a total order $>, \sim$ on $X$ such that $P \subset \succ$.

(v) $P^{*}$ is igreflexitue.

Proof: (i) $\Rightarrow$ (ii): Any weak order is a preorder. 


\section{Apperadix}

(ii) $=$ (iii): Use Theorem A.3.

(iii) $\Rightarrow$ (iv): Use theorm $A, \mathbb{1}$.

$(v) \Rightarrow(v):$ If $P C>$ then, by transitivity of $\succ$, one has $P^{*} c \succ$. Hence $P^{*}$ is drreflexive, because $>$ is irreflexive.

$(v) \Rightarrow$ (i): If $P^{*}$ is irreflexive then clearly $P^{*} \cap P^{c}=\sigma$. Hence condition (iii) in Theorem $A .4$ is satisfied, if we take $R=\emptyset$. So this theorem implies the existence of a preorder $>, \sim$ on $X$ such that $Q \subset z$ and $P \subset>$. 


\section{B GRAPH THEORY}

\section{B.1 Introduction}

The graph theory in this appendix is related to a revealed preference type of problem, treated in Appendix A. This problem is the question whether there exists a preorder $\succ$, such that $P \subset>$, given a binary relation $P$. We can represent the relation $P^{\text {as }}$ a graph, a set of arrows between points. Then $x P y$ means that there is an arrow from $x$ to $y$. Now, the solution of the problem is the following. The desired preorder exists if and only if we can never return to the same point, when following arrow paths in the graph of $P$.

Another problem occurs when there does not exist a preorder $\succ$, such that PC>. Then it is the question how we can find a minimal set of points that can be deleted, together with its arrows, in order to find a smaller graph for which such a preorder exists. Such a set is called a minimal feedback vertex set. The problem of finding a minimal feedback vertex set is related to the deletion of a minimall number of observations in order to get consistency with the axiom of revealed preference.

\section{B.2 Graphs}

A graph can be depicted by a diagram in which uertices are represented by points in the plane, and each arc $(x, y)$ is represented by an arrow drawn from the point representing $x$ to the point representing $y$.

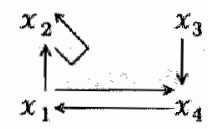

Figure B.1 A Graph

So a graph $G=(X, U)$ is a set $X$ of vertices together with a subset $U$ of the Cartesian product $X \times X$, whose elements are called arcs. For example, Figure B.1 represents the graph $G=(X, O)$ in which

$$
\begin{aligned}
& X=\left\{x_{1}, x_{2}, x_{3}, x_{4}\right\}, \\
& U=\left\{\left(x_{1}, x_{2}\right),\left(x_{1}, x_{4}\right),\left(x_{2}, x_{2}\right),\left(x_{3}, x_{4}\right),\left(x_{4}, x_{1}\right)\right\} .
\end{aligned}
$$

There is a close relation between graphs and binary relations. Any binary relation $R$ on a set $X$ defines a graph $G=(X, R)$ and vice versa.

Another representation of a graph $G=(X, U)$ with vertices $X=\left\{x_{1}, \ldots, x_{n i}\right\}$ is given by its $n \times n$ adjocency matrix $A=\left[a_{i j}\right]$, which is a Boolean matrix defined by

$$
a_{i j}= \begin{cases}1 & \left(\left(x_{i}, x_{j}\right) \in U\right), \\ 0 & \left(\left(x_{i}, x_{j}\right) \notin U\right) .\end{cases}
$$

For example, the adjacency matrix of the graph in Figure B.1 is the Boolcan matrix 


$$
A=\left[\begin{array}{llll}
0 & 1 & 0 & 1 \\
0 & 1 & 0 & 0 \\
0 & 0 & 0 & 1 \\
1 & 0 & 0 & 0
\end{array}\right] .
$$

The adjacency matrix provides a convenient form to store a graph in a computer.

The teminology of grapti theory is not standardized. As described above I consider graphs $G=(X, U)$ in which no multiple arcs may occur, i.e. no element of $X \times X$ appears more than one time in $U$. However, loops $(x, x)$ may occur. Some authors prefer to use the terms 'linear oriented graph', 'directed graph', or 'digraph' for the above definition of a graph, and often the term "node" is used rather than sertex".

When considering a graph $G=(X, v)$ we use the following terminology: If $u=(x, y)$ is an element of $U$ then $t$ is called incident from $x$ and incident to $y$. furthermore, $x$ is called a predecessor of $y$ and $x$ a successor of $y$. Any vertex $x$ which is end point of a loop $(x, x)$ is called an essential vertex.

\section{B.3 Subgraphs}

When we cut out a piece of a graph we are left with a subgraph. More precisely, if we remove from a $\mathrm{graph} G=(X, U)$ a subset of its vertices together with all the arcs incident to or from those vertices, we are left with a graph of the form $G_{Y}=\left(Y, U_{Y}\right)$, where $Y \subset X$ and $U_{Y}=U \cap Y \times Y$. Such a graph is called a subgraph of $G$. We may describe $G_{Y}$ more precisely as the subgraph of $C$ generated by $Y$. In case one has $Y=X-\{x\}$ we also use the notation $G_{x}$ instead of $C_{Y}$

\section{B.4 Paths in a Graph}

When we follow the arrows in a graph then we are following a path. So a path on a graph $G=(X, U)$ is a finite sequence of $\operatorname{arcs} u_{1}, \ldots, u_{r}$ in $U$ of the form

$$
\left(x, x_{i_{1}}\right),\left(x_{i_{1}}, x_{i_{2}}\right), \ldots,\left(x_{i_{r}}, y\right)
$$

One says that this is a path from $x$ to $y$, and this path encounters every vertex in the sequence.

If the end points $x$ and $y$ coincide then the patl is called a cycle. A grapl is called an acyclic graph if it does not contain any cycle.

\section{B.5. Strongly Connected Vertices}

Strongly comected vertices are connected to each other by a cycle path and a strongly connected component is a strongly connected set of vertices. More precisely:

Let $G=(X, y)$ be a graph. A pair of vertices $x, y \in X$ is said to be strongly connected if there are paths from $x$ to $y$ and from $y$ to $x$. The graph $G$ is said to be a strongly connected graph if each pair of distinct vertices $x, y \in X$ is strongly connected.

Now, let us define the binary relation $\sim$ on $X$ by

$$
x \sim y \Leftrightarrow x=y \text { or the pair } x, y \text { is strongly connected }(x, y \in X) .
$$

This relation is clearly an equivalence relation. The classes of this equivalence relation partition $X$ into subsets which generate strongly connected subgraphs of $G$. These subsets are called the strongly connected components of $G$. For example, the strongly connected components of the graph 
in Eigure $\mathbb{B} .1$ are $\left\{x_{1}, x_{4}\right\},\left\{x_{2}\right\}$ and $\left\{x_{3}\right\}$.

\section{B.6 Absorption of a Vertex}

To absorb a vertex in a graph, we pull out the vertex and connect the outgoing and incoming arrows with each other. More precisely:

Let $G=(X, U)$ be a graph and let $x \in X$. Define the graph $G_{x}^{*}=\left(X^{*}, V^{\prime}\right)$ as follows:

$$
X^{\prime}=X-\{x\}, U^{\prime}=\left\{(y, z) \in X \times X^{\prime} \mid(y, z) \in U \text { or }(y, x),(x, z) \in U\right\} .
$$

The graph $G_{x}^{*}$ is said to be obtained by the absorption of vertex $x$.

The following theorems concem the absorption of a vertex in graph. The elementary proofs are left to the reader.

Theorem B.1: Let $G=\left(X_{y} U\right)$ be a graph. Suppose $x \in X$ is not an essemtial vertex. Then $G$ is acyclic if and only if $G_{X}^{\prime}$ is acyclic.

Theorem B.2: Let $G=(X, U)$ be a graph. Then

$$
\left(G_{x}^{*}\right\rangle_{Y}=\left(G_{Y}\right)_{x}^{*} \quad(x \in Y \subset X) \text {. }
$$

\section{B.7 Feedback Vertex Sets}

When we cut out a reedback vertex set then the resulting graph is acyclic. More precisely, given a graph $G=(X, t)$ we say that a subset $Y$ of $X$ is a feedback yertex set of $G$ if every cycle in $G$ encounters at least one vertex in $Y$. So a feedback vertex set has the following property.

Theorem B.3: Let $G=(X, U)$ be a graph and $Y \subset X$. Then $Y$ is a feedback wertex set of $G$ if and only if $G_{X-Y}$ is an acychic graph.

Proof: Obvious.

We denote the set of all feedback vertex sets of $G$ as $\mathcal{F}(G)$. A feedback vertex set $Y \in \mathcal{F}(G)$ of minimal cardinality is called a minimal feedback vertex set.

The following theorems are valid for feedback vertex sets. The first theorem says that in case we search for all feedback sets containing a certain vertex, we may remove this vertex and search further.

Theorem B.4: Let $G=(X, C)$ be a graph and let $x \in X$. Then

$$
\{Y \in \mathcal{F}(G) \mid x \in Y\}=\left\{Y \cup\{x] \| Y \in \mathcal{F}\left(G_{x}\right)\right\} \text {. }
$$

Proof: Let $x \in Y \subset X$. Then one has obviously $G_{X-Y}=\left(C_{X}\right)_{X-Y}$. So the result follows from Theorem B.3.

At the other hand, when we search for all leedback sets not containing a certain vertex, we may absorb this vertex and search further.

Theorem B.5: Let $G=(X, U)$ be a graph and $x \in X$ not an essential wertex. Then $\{Y \in \mathcal{F}(G) \mid x \notin Y\}=\mathcal{F}\left(G_{x}\right)$.

Proof: Let $x \notin Y \subset X$. Then Theorem $B .1$ implies that $G_{X-Y}$ is acyclic if $\left(G_{X-Y}\right)_{*}$ is acyclic. Furthermore, by Theorem B.2 one has

$$
\left(G_{X-Y}\right)_{X}^{\prime}=\left(G_{X}^{s}\right)_{X-Y}
$$

Thus the result follows from Theorem B.3.

We combine both above theorems to prove the following main theorem. This theorem is used by Guardabassi (1971) in a branch-and-bound algorithm to find minimal feedback vertex sets.

Theorem B.6: Let $G=(X, U)$ be a graph and $x \in X$ not an essential vertex. Then 


\section{Appendix}

$$
\mathcal{F}(G)=\left\{Y \cup\{x) \mid Y \in \mathcal{F}\left(G_{x}\right)\right\} \cup \mathcal{F}\left(G_{x}^{*}\right)
$$

Proof: Follows immediately from Theorem B.4 and B.5.

Thus in case we are searching for minimal feedback sets, we may split the problem in wo maller problems using the above theorem. Furthermore, we may simplify the problem by only considering the strongly connected components. 


\section{PATH ALGEBRA'S AND LABELLED GRAPHS}

\section{C.I Introduction}

In Carré (1979) the definition of a graph is generalized, using a path algebra, which allows for a more unified treatment of path problems. The reason I introduce this idea is that it clarifies the connection between the two following problems:

(a) Given a relation $P$ on $X$, is there a preorder $>, \sim$ such that $P \subset>$ ?

(b) Given a square matrix $A$, are there numbers $\phi_{i}$ such that

$$
\phi_{i}-\phi_{j} \leq a_{i j} \quad(i, j \in I) \text {. }
$$

Problem (a) is related to acyclic Boolean graphs and is treated in Appendix B. Problem (b) is related to shortest paths in a labelled graph. The connection of the problem (a) and (b) becomes clear when we use a path algebra. A similar connection is walid for the following two problems:

(a) Given $R$ and $P$, is there a preorder $>$, such that

$$
R \subset \succsim, P \subset>\text {. }
$$

(b) Given $A$ and $B$, are there numbers $\phi_{i}$ such that

$$
\phi_{i}-\phi_{j} \leq a_{i j}, \phi_{i}-\phi_{j}<b_{i j} \quad(i, j \in I) .
$$

Problem (a') is solved in Appendix $A$, which considers binary relations, but can also be translated into a graph problem. Both problems (b) and $\left(b^{\prime}\right)$ are solved in this appendix, using path algebra's. Carre's definition of a path algebra is repeated in the following section for convenience.

\section{C.2 Path Algebra}

A path algebra is a set $p$ equipped with two binary operations $v$ and $\cdot$ which have the following properties, for all $x, y, z \in P$ :

1. The $v$ operation is idempotent, commutative, and associative:

$$
\begin{aligned}
& x \vee x=x, \\
& x \vee y=y \vee x, \\
& (x \vee y) \vee z=x \vee(y \vee z)
\end{aligned}
$$

2. The - operation is associative, and distributive over $v$ :

$$
\begin{aligned}
& (x \cdot y) \cdot z=x \cdot(y \cdot z), \\
& x \cdot(y \vee z)=(x \vee y) \cdot z=(x \cdot y) \vee(y \cdot z) .
\end{aligned}
$$

3. The set $p$ contains a zero element $\emptyset$ such that

$$
\begin{aligned}
& \emptyset \vee x=x, \\
& \emptyset \cdot x=x \cdot \emptyset=\emptyset,
\end{aligned}
$$

and a unit element, $e$ such that

$$
e \cdot x=x \cdot e=x \text {. }
$$

The operation $v$ is called the join operation and the operation is called multiplication. Matrix operations are defined as follows:

$$
X \vee Y=\left[x_{i j}, y_{i j}\right],
$$




$$
X \cdot Y=\left[V_{k} x_{i k} \times y_{k j}\right]
$$

The notation $x^{k}$ and $x^{k}$ have the meaning of a repeated multiplication as in

$$
x \cdot x=x^{2}
$$

The binary relation $\leq$ on $P$ is defined as

$$
x<y \Leftrightarrow x \vee y=y \quad(x, y \in P) \text {. }
$$

As the reader may verify this is a reflexive and antisymmetric relation, and thus artial order, on $P$. Simlarly, for matrices we define

$$
X \preceq Y \div X \vee Y=Y \text {. }
$$

\section{C.3 Examples of Path Algebra's}

We shall consider the following path algebra's?

1. The Boolean path algebra, where

$$
\begin{aligned}
& P=\{0,1\}, \quad=0, \quad e=1, \\
& x \vee y=(x \text { or } y), x \cdot y=(x \text { and } y) .
\end{aligned}
$$

2. The shortest path algebra, where

$$
\begin{aligned}
& P=\mathbb{R} \cup\{\infty\}, \varnothing=\infty, e=0, \\
& x \vee y=\min \{x, y\}, x \cdot y=x+y .
\end{aligned}
$$

Note that the notation is a bit confusing for the shortest path algebra, because one has then $x \leq y$ equivalent to $x \geq y$.

As the reader may verify, the Boolean path algebra can be represented as a subset of the shortest path algebra. "This is done by using the bijective. mapping

$$
I I(0)=\infty, \quad I(1)=0
$$

One has for example

$$
\Pi(x \text { and } y)=\min \{\Pi(x), \Pi(y)\}, \Pi(x \text { or } y)=\Pi(x)+\Pi(y)
$$

\section{C.4 Labelled Graphs}

Carré (1971) defines a labelled graph as a graph $G=(X, b)$ together with a labeling $l: U \rightarrow P-\{\varnothing\}$, where $P$ is a path algebra. I will extend for convenience the labelling $t$ on $X \times X$ by assuming $l(u)=\varnothing$ for $u \& U$, so that a labelled graph is tepresented as $l: X \times X \rightarrow p$.

Any labelled graph o with $n$ vertices can be described by its nxn adjacency mawrix $A=\left[a_{i j}\right]$ with entries $a_{i j}=l\left(\left(x_{i}, x_{j}\right)\right)$. For example, concerning a graph labelled with the Boolean path algebra one has $l(u)=1$, for $u \in U$. Because $\varnothing=0$, we obtain in this case the usual Boolean adjacency matrix.

An example of abelled graph for the shortest path algebra is given in Figure C.I.

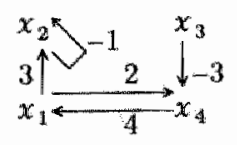

Figure C.1 A Labelled Graph 
The adjacency matrix of the labelled graply in figure C.1 is:

$$
A=\left[\begin{array}{cccc}
\infty & 3 & \infty & 2 \\
\infty & -1 & \infty & \infty \\
\infty & \infty & \infty & -3 \\
4 & \infty & \infty & \infty
\end{array}\right]
$$

We can generalize the definitions, concerned with graphs, easily for application to labelled graphs. The term "labelled graph" will often be abbreviated to "graph" for convenience.

Let $l: X \times X \rightarrow P$ be a labelled graph. The subgraph of $l$ generated by $Y \subset X$ is the graph $l_{Y}=l \mid Y$. The length (or cost) of a path $\mu=u_{1}, u_{2}, \ldots, u_{r}$ is

$$
l(\mu)=l\left(u_{1}\right) \cdot d\left(u_{2}\right) \cdot \ldots \cdot l\left(u_{r}\right)
$$

A labelled graph $l$ is said to be absorptive if one has

$$
(y) \precsim e
$$

for every cycle $\gamma$. The adjacency matrix of a graph is said to be absorptive, when the corresponding labelled graph is absorptive.

We might use the following definition for an acyclic labelled graph. A labelled graph is acyclic when $l(\gamma)<e$ for every cycle $\gamma$. Note that any graph labelled with the Boolean algebra is absorptive, but not necessarily acyclic.

For absorptive adjacency matrices one can prove the following theorem.

Theorem C.1: Suppose $A$ and $B$ are adjacency matrices and $A \precsim B$. If $B$ is absorptive then $A$ is absorptive.

Proof: See Carre (1979, page 105).

\section{C.5 The Weak Closure of an Adjacency Matrix}

The weak closwre of an adjacency matrix $A$ is defined as

$$
A^{*}=\lim _{n \rightarrow \infty} \bigvee_{k=1}^{n} A^{n}
$$

In case of the Boolean algebra the limit results in the transitive closure of the binary relation corresponding to $A$. In case of the shortest path algebra the limit $a_{i j}^{*}$ is equal to the shortest path length taken over all paths from vertex $x_{i}$ to $x_{j}$. A quick way to compute transitive closures is introduced by Warshall (1962) in relation to Boolean matrices. This algorithm is also described by Floyd (1962), but now with the aim to determine shortest path
lengths. Both algorithms are identical, when considered in path algebra terms, and as follows:

For all $\imath \in I$ do

For all $j \in l$ do

For all $k \in I$ do

$$
\text { Set } a_{i j}=a_{i k} \vee a_{k j} \text {. }
$$

This simple algorithm transforms adjacency matrix $A$ into the weak closure $A^{*}$. For the shortest path algebra one has $a_{i j}^{*} \in[-\infty, \infty)$, because the matrices

$$
\bigcup_{k=1}^{m} A^{n} \quad(n \in \mathbb{N})
$$

are decreasing for $n \rightarrow \infty$. So it may happen that $a_{i j}^{*}=-\infty$, thus $A^{*}$ is not necessarily a well-defined adjacency matrix for the shortest path algebra. Furthermore, in that case the algorithm given above is not reliable. However, 
the following theorem is vallid.

Theorem C.2: Let $A$ be the adjucency matrix of a graph labelled ath the shortest path olgebra and with vertices $\left\{x_{i}\right\}_{\text {ie }}$. Suppose (a) or (b) satssfited:

(a) $a_{i j} \in \mathbb{R} \quad(i, j \in I)$

(b) A finute-dimensional.

Then the following conditions are equivalent:

(1) A is absorptive.

(ii) $A$ is a well-defined adjacency motrix.

(iii) There exst numbers $\phi_{i}$ such that

$$
\phi_{i}+\phi_{j} \leq a_{i j} \quad(i, j \in I)
$$

Proof (i) $\Rightarrow$ (ii): Suppose $A$ is an absorptive matrix. To show that $A^{\text {*h }}$ is a well-defined adjacency matrix it is sufficient to show

$$
a_{i j}^{*} \neq-\infty \quad(i, j \in I) \text {. }
$$

So let $b_{y} j \in I$ and suppose $a_{i j}^{*}=-\infty$. We have to show this is impossible.

First, suppose (a) is satisfied. Since $a_{i j}^{*}=-\infty$, there exists a path $\mu$ from $i$ to $j$ such that $l(\mu)+a_{j i}<0$. Hence $l(\gamma)<0$, for the cycle $\gamma=\mu,\left(x_{j}, x_{i}\right)$, contradicting the assumption that $A$ is absorptive.

Now, suppose (b) is satisfied. Since $a_{i j}^{*}=-\infty$, there exists a sequence of paths $\mu_{n}$ from $x_{i}$ to $x_{j}$, such that

$$
\lim _{n \rightarrow \infty} l\left(\mu_{n}\right)=-\infty \text {. }
$$

Construct a sequence paths $\mu_{\eta}^{\prime \prime}$ without cycles by removing all cycles from the paths $\mu_{n}$. The resulting sequence satisfies again

$$
\lim _{n \rightarrow \infty} l\left(\mu_{n}^{\prime}\right)=-\infty
$$

because each removed cycle is of nonnegative cost, since $A$ is absorptive. Hence the number of arcs approaches infinity when $n \rightarrow \infty$. Now, all arcs in $\mu_{n}^{*}$ are different, because otherwise there is a cycle left. So we obtain a contradiction with (b), because there are maximal $m^{2}$ different arcs in a graph with $m$ vertices.

(ii) $\Rightarrow$ (iii): Suppose $A^{*}$ is a well-defined adjacency matrix. Then one has

$$
a_{i k}^{*} \leq a_{i j}+a_{j k}^{*},-\infty<a_{i j}^{*} \leq a_{i j} \quad(k, i, j \in I) \text {. }
$$

Suppose now that (a) is satisfied. Let $k \in I$ and define $\phi_{i}=a_{i k}^{*}$ for all $i \in I$. Then clearly $\phi_{i} \in \mathbb{R}$, for every , and

$$
\phi_{i}-\phi_{j} \leq a_{i j} \quad(i, j \in I) \text {. }
$$

Now, suppose (b) is satisfied. Then the above proof cannot be used, because we may have $a_{i k}^{*}=\infty$. To awoid this, let us define the following adjacency matrix

$$
b_{i j}= \begin{cases}\alpha & \left(a_{i j}=\infty\right), \\ a_{i j} & \text { (otherwise) }\end{cases}
$$

Because $A^{*}$ is a well-defined adjacency matrix and finite-dimensional, we may choose $\alpha$ such that $B^{*}$ is well - defined. Otherwise we can show the existence of a cycle on $B$, which is of negative length for every $\alpha \in \mathbb{R}$. (For the latter we need the assumption that $A$ is finite-dimensional). Such a cycle will be of negative length on $A$, which contradicts the assumption that $A^{*}$ is a well-defined adjacency matrix. 
Now, because $B^{*}$ is well-defined and satisfies condition (a) we may apply the previous results, derived for the case that (a) is satisfied, to obtain

$$
\phi_{i}-\phi_{j} \leq b_{i j} \leq a_{i j} \quad:(i, j \in I)
$$

(iii) $\Rightarrow$ (i): Define the adjacency matrix $B$ as follows

$$
b_{i j}=\phi_{i}-\phi_{j} \quad(\dot{i}, j \in I) .
$$

Then obviously $l(\gamma)=0$ for any cycle path $\gamma$ on the graph of $B$, thus $B$ is absorptive. Furthermore, (iii) implies that we have $A \lesssim B$. Hence $A$ is absorptive by Theorem C.1.

Let us define an adjacency matrix $A$ irreflex we when $a_{i i} \neq$ for all i.

Theorem C.3: Let $A$ and $B$ be two finite-dimensional adjacency matrices of $a$ graph labelled with the shortest path algebra and with vertices $\left\{x_{i}\right\}_{\text {ier }}$. Then the following conditions are equivalent:

(i) There exist numbers $\phi_{i}$ such that

$$
\phi_{i}-\phi_{j} \leq a_{i j}, \phi_{i}-\phi_{j}<b_{i j} \quad(i, j \in I) \text {. }
$$

(ii) $A \vee B$ is absorptive and $(A \vee B)^{*} \cdot B$ is irreflexive.

Proof (i) $\Rightarrow$ (ii): Put $C=A \vee B$. That $C$ has to be absorptive follows from Theorem C.2. Further, because one has

$$
\phi_{i}-\phi_{j} \leq c_{n j}^{*}, \phi_{j}-\phi_{i}<b_{j i} \quad\left(i_{j} j \in I\right),
$$

it follows that one has $c_{i j}^{*}+b_{j i}>0, i, j \in I$. Hence $C^{*} \cdot B$ is irreflexive.

(ii) $\Rightarrow$ (i): Put $C=A \vee B$ and suppose $C \in \mathbb{R}^{m}$. Because $C$ is absorptive, $C^{*}$ is a well-defined adjacency matrix by Theorem C.2. Now, put.

$$
\phi_{i}=m^{-1} \sum_{k \neq i} c_{i k}^{*} \quad(i \in I) .
$$

The definition of $C^{*}$ implies now

$$
c_{i k}^{*}-c_{j k}^{*} \leq c_{i j}, c_{i j}^{*} \leq c_{i j},-c_{j i}^{*} \leq c_{i j} \quad(k, i, j \in I) .
$$

Thus we have

$$
\phi_{i}-\phi_{j}=m^{-1}\left(\left[\sum_{k \neq i, j} c_{i k}^{*}-c_{j k}^{*}\right]+c_{i j}^{*}-c_{j i}^{*}\right) \leq m^{-1} m c_{i j}=c_{i j} \leq c_{i j} \quad(i, j \in I) .
$$

Finthermore, $C^{*} \cdot B^{*}$ irreflexive implies $c_{j i}^{*}+b_{i j} \neq 0$ for all $i, j \in l$. From this and $-c_{j i}^{* k} \leq c_{i j} \leq b_{i j}$ we obtain that

$$
-c_{j i}^{*}<b_{i j} \quad(i, j \in I) \text {. }
$$

Hence, the inequalities in condition (i) are satisfied, because we have

$$
\phi_{i}-\phi_{j} \leq m^{-1}\left((m-1) c_{i j}-c_{j i}^{*}\right)<m^{-1} m b_{i j}=b_{i j} \quad\left(i_{i j} \in l\right)
$$

Now suppose that we may have $c_{i j}=\infty$. In that case we can derive the resilt in a. similar way as in the proof (ii) $\Rightarrow$ (iii) of Theorem C.2. This is done by replacement of all infinite values in $A$ and $B$ by a finite value $\alpha$, such that condition (ii) remains valid. Then the result follows from the above proof in case of finite values.

There is an interesting connection between the above theorem and Theorem A.A. Condition (iii) in Theorem $A 4$ was $(R \cup P)^{*} \cap P^{C}=\varnothing$, where $R$ and $P$ are binary relations. When we identify these relations with their Boolean matrices, this is clearly equivalent to the condition that $(R \vee P)^{*} \cdot P$ is irreflexive. But this is exactly what appears as condition (ii) in Theorem C.3. Note that $R \vee P$ is absorptive as any Boolean matrix is absorptive.

We can explain this resemblance by representing the Boolean path algebra as a 
shortest path algebra, using, the mapping

$$
I(0)=\infty, \pi(1)=0
$$

Now the following clarifies why Theorem A.4 is a special case of Theorem $\mathrm{C} 3$. The existence of weak order $>$ such that

$$
R \subset \geq, P C \succ \text {. }
$$

in Theorem $A, 4$ is in fact equivalent to the existence of numbers $\phi_{i}$ such that

$$
\phi_{i}-\phi_{j} \leq a_{i j}=\Pi\left(x^{i} R x^{j}\right), \phi_{i}-\phi_{j}<b_{i j}=\Pi\left(x^{i} P x^{j}\right) \quad(i, j \in I) .
$$

\section{C.6 Theorems for Labelled Graphs}

We may apply the approach in Theorem B.1 to B.6 to feedback sets in labelled graphs. First I give some similar definitions concerning labelled graphs as I have eatlier given for normal graphs.

The labelled graph $l_{x}^{\prime}: X^{\prime \prime} X^{\prime \prime} \rightarrow p$, obtained from absorption of a vertex $x$ in a labelled graph $l: X \times X \rightarrow P_{*}$ is defined by:

$$
\begin{aligned}
& X=X-\{x)_{i} \\
& \left.\left.\left.l_{x}^{\prime}(y, z)\right)=U(y, z)\right) \vee[u(y, x)) \cdot u(x, z)\right] \quad\left((y, z) \in X \times X^{\prime}\right) .
\end{aligned}
$$

Note that this definition is a generalization of the definition for the absorption of a vertex in a Boojean graph, given in Appendix B.

Given a labelled graph $l: X \times X \rightarrow P$, a subset $Y$ of $X$ is said to be an feedbcck wertex set if every cycle $\gamma$, such that

$$
l(\gamma) \leq e
$$

encounters at least one vertex in $Y$. The set of all feedback vertex sets for $l$ is denoted by $A(l)$. A vertex $x \in X$ is said to be an essential vertex when $l((x, x)) \leq e$

We may proof the following six theorems, which are straightforward generalizations of the theorems B.1 until B.6.

Theorem C.4: Let $l: X \times X \rightarrow p$ be a labelled graph and suppose that $x \in X$ is not an essential vertex. There $l$ is absorptive if and only if $l_{x}$ is absorptive.

So, if we do wish to test whether a labelled graph $l$ is absorptive, we may derive from Theorem C.4 the following algorithm:

Step 1. Set absorptive $=$ True.

If $l$ contains an essential vertex then put absorptive - False.

Step 2. While absorptive $=$ True and $t$ contains more then one vertex do the following:

Take an arbitrary vertex $x$ of $l$ and put $l=l_{x}^{\prime}$.

If $l$ contains an essential vertex then put absorptive $=$ False.

The result absorptive will contain the answer whether is absorptive or not.

Theorem C.5: Let $4: X \times X \rightarrow p$ be a labelled graph. Then

$$
\left(l_{x}^{\prime}\right)_{y}=\left(h_{y}\right)_{x}^{\prime} \quad(x \in Y \subset X) \text {. }
$$

Theorem C,6: Suppose $4: X \times X \rightarrow P$ is labelled graph and $Y \subset X$. Then $Y$ is an feedback vertex set of $l$ if and only of $l_{X-Y}$ is absorptive.

Theorem C.7: Suppose $\quad: X \times X \rightarrow P$ is a labelled graph and $x \in X$. Then

$$
\left\{Y^{\prime} \in \mathcal{A}(l) \mid x \in Y\right\}=\left\{Y \cup\{x\} \mid Y \in A\left(b_{x}\right)\right\}
$$


Theorem C.8: Suppose $l: X \times X \rightarrow p$ is a labelled graph and $x \in X$ is not an essential vertex. Then

$$
\{Y \in \mathcal{A}(b) \mid x \notin Y\}=\mathcal{A}\left(l_{x}\right)
$$

Theorem C.9: Suppose $l: X \times X \rightarrow P$ is a labelled graph and $x \in X$ is not an essentinul vertex. Then

$$
\mathcal{A}(l)=\left\{Y \cup\{x\} \mid Y \in A\left(l_{x}\right)\right) \cup \mathcal{A}\left(l_{x}^{*}\right)
$$

The above theorem can be used as a branch rule in a branch-and-bound algorithm which determines minimal feedback sets in a labelled graph. 


\section{LAPLACE TRANSFORMS}

The (bilateral) Laplace transform $\vec{f}: C \rightarrow C$ of a function $f: \mathbb{R} \rightarrow \mathbb{R}$ is defined as

$$
\tilde{f}(s)=\int_{-\infty}^{\infty} f(t) \mathrm{e}^{-u t} d t
$$

The Laplace transform $\tilde{f}$ is only well-defined at $s \in \mathbb{C}$, if the above integral is well-defined. When a Laplace transsform is well-defined in the strip $a \leq R e s \leq b$, then the Laplace transform is analytic in this region and the inverse of the Laplace transform is given by

$$
f(t)=(2 \pi)^{-1} \int_{c-i \infty}^{c+i \infty} \tilde{f}(s) e^{s t} d s \quad(a<c<b) .
$$

The notations $\tilde{f}=\mathcal{L}[f]$ and $f=\mathcal{L}^{-1}[\tilde{f}]$ are used to denote the Laplace transform and the inverse of the Laplace transform respectively. One has at a simple discontinuity $t$ of the function $f$

$$
\mathcal{L}^{-1}[\mathcal{L}[f]](t)=\frac{1}{2}\left(f\left(t^{+}\right)+f\left(t^{-}\right)\right)
$$

The convolution theorem states the following useful property of convolutions.

Theorem D.1: Suppase the Laplace transforms of two functions $f(\cdot)$ and $g(\cdot)$ well-defined in a wertical strip in the complex plain. Then the convolution product $f_{*} g$ is well-defined. Furthermore, for $s$ in this vertical strip the Laplace transform of $f_{* g} g$ is equal to $\tilde{f}(s) \tilde{g}(s)$ and well-defined.

Table D.1 Examples of Laplace transforms

\begin{tabular}{ll}
\hline Function & Laplace transform \\
$f_{* g}(t)$ & $\tilde{f}(s) \tilde{g}(s)$ \\
$\int_{-\infty}^{t} f(\tau) d \tau$ & $s^{-1} \tilde{f}(s)$ \\
$\frac{d}{d \tilde{t}} f(t)$ & $s \tilde{f}(s)$ \\
$e^{-a t} f(t)$ & $\tilde{f}(s+a)$ \\
$f(t-a)$ & $e^{-a s} \tilde{f}(s)$ \\
$\delta(t)$ & 1 \\
$1(t) t^{n-1} /(n-1) !$ & $s^{-n}$ \\
\hline
\end{tabular}




\section{E DESCRIPTION OF THE DUTCH INDUSTRY DATA}

\section{E.1 Prices and Quantities}

The input data of the Dutch industry consist of prices and quantities of the commodities that are given in Table $\mathbb{E}_{1}$. The sectors in this table are used by the Netherlands Central Planning Bureau. The Dutch industry output data. consist of aggregate total output data for the sectors $2-13$; excluding indirect taxes and including subsidies. So when I refer to "Dutch industry" in this book I mean the aggregation of the sectors $2-13$.

Table E.1 Inputs of the Dutch Industry

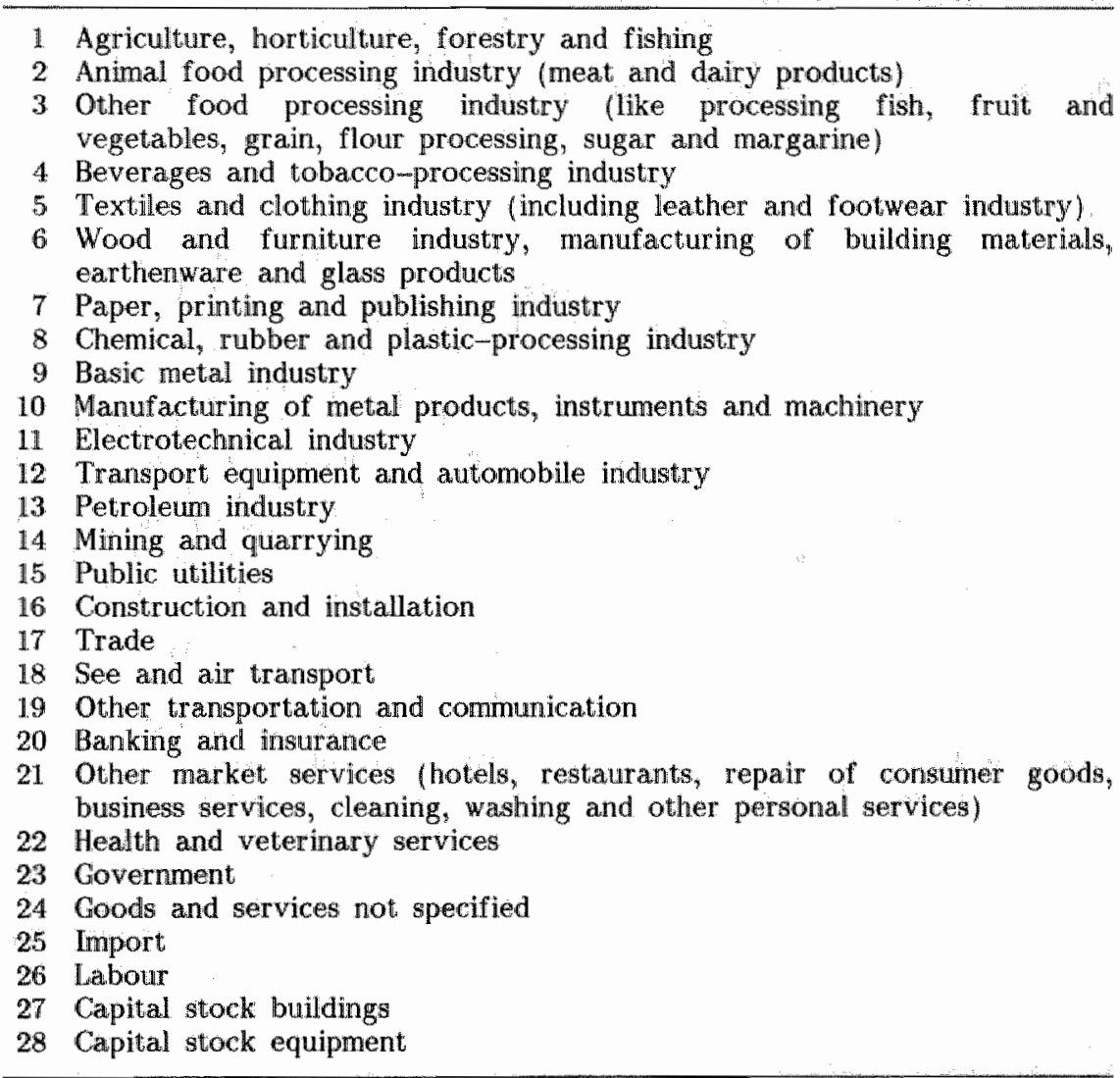

The above table looks simple, but it was quite a lot of work to gather a complete data set. I have constructed complete input-output data sets concerming the twelve industry branches $2-13$, including capital data. In this book II describe only the results concerning the aggregate data, generated using Paasche price indices, because it was not my aim to write an empirical study about Dutch industry branches.

The main source of nominal data were input-output tables published by the 
Netherlands Central Bureaus of Statistics in the National Accounts. These publications also contain tables of taxes and subsidles. These tables are used to derive nominal sales data, excluding indirect taxes and including subsidies. Only for recent years there were a few deflated input-output tables available, so it was necessary to gather price data for the nominal data. Furthermore, the input-output tables do not contain data on capital formation by destination, a problem that will be discussed in the following section.

The input output tables were deflated, using price data from various sources. A main source of price data was the Central Economic Plan published by the Metherlands Central Planning Bureau. To obtain accurate price data for the input commodities the following publcations of the Netherlands Central Bureau of Statistics are used: Maandstatistiek van de Prijzen, Maandstatistick wan de Binnenlandse Handel, Statistical Yearbook of the Netherlonds, and Prijsanalyse. The output prices of the Central Planning Bureau were used in case input prices were not available. Further, labour volume data was also obtained from the Central Planning Bureau. In labour volume the self-employed are included. The nominal cost of labour, given in the input-output tables and excluding the self-employed, is proportionally increased.

\section{E.2 Capital Stocks}

In this section I describe briefly the way I have generated the capital stocks concerning the Dutch industry data, which are used as a data example in this book. To generate capital stocks there were a few problems, which had to be solved. First I needed the mean and the variance of the lifetime of investments. I obtained from the Netherlands Central Bureau of Statistics parameter values for the gamma distribution for several types of investment goods at a low aggregation, level. For these parameters I computed the corresponding mean and variance. However, the investment data for the generation of the capital stock was only available at a higher aggregation level. Therefore an 'aggregate' mean and variance had to be estimated. These were obtained from an availabie data series of expenditures for investment goods at a low aggregation level for the years 1969-1984, given in Statistics on Fixed Capital Formation in Industry published by the Netherlands Central Bureau of Statistics. From these data and the available mean and the variance of the lifetime of a money unit investment at a low aggregation level, I estimated the mean and variance of the lifetime of a money unit investment at a higher aggregation level.

For the obtained mean and variance of the investment lifetime there is a corresponding contaglous Erlang retirement distribution, as is described in Section 19.2. For this distribution a corresponding state-space realization is described in Section 19.3. I generated the capital stocks with the discrete-time model in Section 19.5 , using this state-space realization. "To generate the capital stocks, which I have used in this book for the years 1969-1983, I had investment data available from the Netherlands Central Planning Bureau for the years 1950-1983.

To start the generation of the capital stocks a second problem had to be solved. The model has to be initialized. For this initialization I estimated the rate of investment growth around 1950. To obtain this rate I used available data of the retirement of investments in $1950-1956$, given in a publication of the Netherlands Central Bureau of Statistics (1957). These data and the investment data yielded a mean retirement rate of investments over the years 1950-1956. 
To estimate the rate of investment growth, I assumed that the retirement probability distribution is a gamma function with the above computed nean and variance. As is described in section 19.4, this assumption yields an estimation of the rate of investment growth. This section also described the initial values, which correspond to a given rate of investment growth.

The above procedure yielded time series of capinal stocks, one for buildings. and one for equipment, for 15 branches of industry. Finally, these stocks were aggregated, using chained Paasche price indices, to capital stocks concerning the entire Dutch industiry.

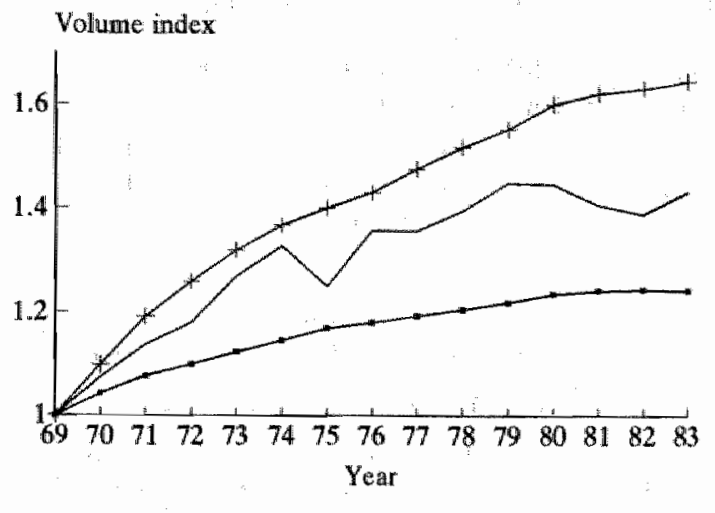

- Buildings + Equipment - Output

Figure E.1 Volume Indices Capital Stocks and Output

\section{E.3 Sensitivity to the Choice of Initial Values}

The sensitiwity of the capital stock data to the choice of initial values depends on the length of the initial period in which the capital stocks are computed, but not actually used as data. The capital stock generation starts in 1950 and the data are used in the period 1969-1983. Hence, the equipment; stocks will not be very sensitive to the way the capitall stocks are initialized. The mean lifetime of equipment is around 15 to 25 year and after 18 years there is not much left of the initialized stock. The mean lifetime of buildings is longer. Hence, the stocks of bullings may contain an error. However, the error due to the choice of initial values will be small, because investments are increasing over time. The capital stocks of bulldings consists in the period $1969-1983$ mainly of irvestments made after 1950 . The contribution of the initialized stock to the capitall stock is after 1969 only approximately $10 \%$ of the total stock.

\section{E.4 User Cost of Capital}

To compute the user cost of capital, I used the methor described in Chapter 18. The retirement distributions were assumed to be gamma functions: These functions are determined by the mean and variance, which are derived as described in the previous section. To compute the discount factor for the 
investment prices, the real interest is calculated as the long-term interest rate mimus the investment price inflation rate. The investment price inflation rate is calculated as the average rate over the past four years. The source of the long-term interest is De Nederlandsche Bank (1985). The investment price data were obtained from the Central Plaming Bureau. The data were corrected to allow for profit tax, investment tax credit and investment premium. The additional data, necessary for this correction, were found in Vermeend (1983), and Gelauff and Hasselman (1985).

It may be interesting for empirical researchers to know that using the conventional assumption of exponental decay would result in a negative user cost of capital for several periods. Of course, this is not a desirable result. It might be a reason why one sometimes increases the user cost of capital by adding an arbitrarily chosen risk premium to the discount factor. In the computation of the user cost of capital, using gamma retirement distributions, however, this problem did not occur. The resulting user cost of capital was positive for all periods.

A negative user cost of capital may occur when the discourted investment price is exponentially increasing, instead of decreasing. Suppose the investment price $p$ at time $t$ is equal to

$$
p(t)=p(0) e^{a t},
$$

where is the rate of price inflation. Now, let $u$ be the profit tax rate and $r$ the interest rate. Then the discounted investment price $p_{I}$ is equal to

$$
p_{I}(t)=p_{r}(0) \mathrm{e}^{-(d-a) t}, d=(1-u) r,
$$

where $d$ is the discount rate. The discount rate $d$ was approximately $4 \%$ to $6 \%$, while the price inflation $a$ often was higher. When this is the case then one obtains an increasing discounted investment price.

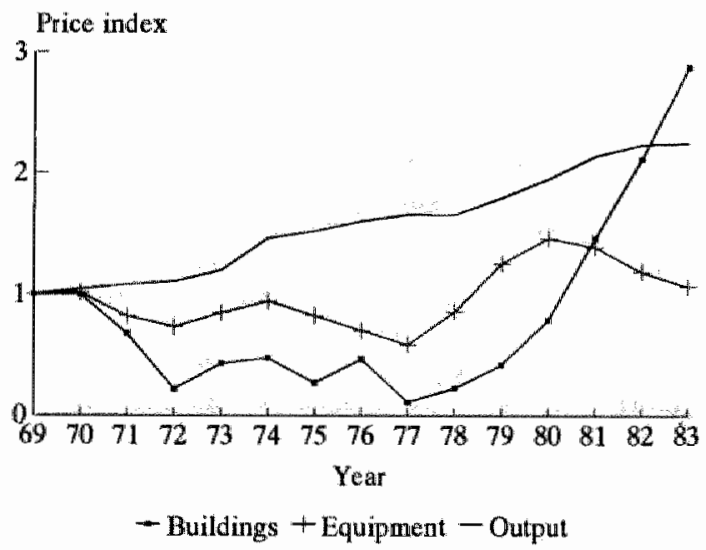

Figure E.2 Price Indices User Cost and Output

In order to check whether the results of the nonparametric tests are strongly influenced by the used assumptions concerning the user cost of capital, the nonparametric tests were applied to several types of user cost of capital 
data: Both the exponential and the gamma retirement distribution were used. Moreover, observed price inflation and profit tax rate were used, and variants in which price inflation or profit tax rate, or both, were ignored and set to zero. This procedure resulted in erght data variants for the user cost of capital. As already remarked, a negative user cost of capital was obtained for the exponential retirement distribution, using price inflation and a profit tax rate. So there remained only seven usable data types, which appeared to produce similar nonparametric test results. Especially the results of efficiency levels and the deletion of obserwations were similar. Concerning the nonparametric bounds, there was only some difference for the neutral change of capital. However, in general the results of the nonparametric tests were only slightly influenced by the used assumptions concerning the user cost of capital. The results described in part If and Part III, concern the assumption of a gamma retirement distribution, using price inflation and profit tax rate. 


\section{F RESULTS OF PRODUCER TESTS}

This appendx contans a summary of results, obtained in Part II and $\mathrm{II}$, of the application of several nomparametric tests to the Dutch industry data. The following table shows the values of $e_{1}$ the upper bound of the economical efficiency level, and the number $n$ of observations, that have to be deleted in order to get consustency with the assumed hypothesis.

Table F.1 Test Results, Observed Outputs Not Used

\section{Existence Production Function}

Cost minimization

Linearly homogeneous cost minimization

Profit maximization $n$

$0 \quad 100.09$

$3 \quad 99.9 \%$

$8 \quad 99.2 \%$

Table F.2 Test Results, Observed Outputs Used

\section{Existence Production Function.}

Cost minimization

Linearly homogeneous cost minimization

Profit maximization $n$

2

13

14

\section{Technical Progress}

Cost minimization

Linearly homogeneous cost minimization

Profit maximization $e$

$99.5 \%$

$89.6 \%$

$81.5 \%$

e

$99.8 \%$

$97.5 \%$

97.18

\section{$e$}

$100.0 \%$

$99.9 \%$

$99.8 \%$

Cost minimization

0

Linearly homogeneous cost minimization 3

Profit maximization

5. $99.8 \%$

\section{Neutral Change of Labour}

Cost minimization

$n \quad e$

Linearly homogeneous cost minimization

0

$100.0 \%$

Profit maximization

$99.8 \%$

$99.3 \%$

\section{Neutral Change of Capital}

Cost minimization

ne

Linearly homogeneous cost minimization

0

Profit maximization 
Table F.2 Continued

Hicks Neutral Progress

Cost minimization $n$

Linearly homogeneous cost minimization

Profit maximization

1

$99.8 \%$

$497.5 \%$

$597,1 \%$

Neutral Progress of Labour

Cost minimization n e

Linearly homogeneous cost minimization

Profit maximization

Neutral Progiess of Capital

Cost minimization $n$

Linearly homogeneous cost minimization

Profit maximization.

$\begin{array}{ll}1 & 99.8 \% \\ 3 & 97.5 \% \\ 3 & 97.1 \%\end{array}$

Weak Separability of Goods and Services

Cost minimization

$n$

2

$99.5 \%$

Linearly homogeneous cost minimization

$13 \quad 89.6 \%$

Profit maximization

$14 \quad 81.5 \%$

Weak Limearly Homogeneows Separability of Goods and Services ${ }^{2}$

Cost minimization

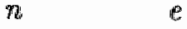

Linearly homogeneous cost minimization

7

Profit maximization

13

Homothetic Function ${ }^{3}$

Cost minimization

Profit maximization

$\begin{array}{rc}n & e \\ 4 & 99.5 \% \\ 14 & -\end{array}$

1. In this case $e$ is an upper bound for the efficiency upper bound.

2 The subfunction is assumed to be linearly homogeneous. There are no efficiency upper bounds avallable for these tests.

${ }^{3}$ While testing the conditions for the existence of a homothetic production function, it appeared that even efficiency level $e=0$ did not satisfy the hypothesis of profit maximization. 


\section{G OVERVIEW OF NONPARAMETRIC TESTS}

Finally, I give an overwiew of several nonparametric tests that are a result: of the main theorems in Part I, II and III. These tests are formulated for application to a finite set of price and quantity data. Such a data description is also used in Afriat (1972, 1981), Hanoch and Rothschildt (1972), Diewert (1973) and Varian (1982, 1983).

\section{G.1 Consumer Demand}

In case of demand behaviour it is assumed that price and quantity data $\left\{\left(p^{i}, x^{i}\right)\right\}_{i \in I}$ are given, where $p^{i}$ and $x^{i}$ are the price and quantity vector for period $:$ respectively. The following list of necessary and sufficient conditions is availlable for hypotheses concerning consumer demand. of course, they can also be applied to producer demand with unobserved output.

Consumer Demand: There exists a function $f$ such that

$$
f\left(x^{i}\right)=\sup _{p^{i} x p^{i} x^{i}} f(x), p^{i} x^{i}=\min _{f(x) \leq f\left(x^{i}\right)} p^{i} x \quad(i \in I)
$$

Condition: Let $R$ and $P$ be defined such that

$$
\begin{aligned}
& p^{i} x^{j} \leq p^{i} x^{i} \Leftrightarrow x^{i} R x^{j}, \\
& p^{i} x^{j}<p^{i} x^{i} \Leftrightarrow x^{i} P x^{j} .
\end{aligned}
$$

Then one must have

$$
x^{i} R^{*} x^{j} \Rightarrow \operatorname{not} x^{j} P x^{i} \quad(i, j \in I) \text {. }
$$

See Theorem 2.5, and also Afriat (1967) and Varian $(1982,1983)$.

Linear Homogeneity: There exists a linearly homogeneous function $f$ which satisfies the demand hypothesis.

Condition: There exist numbers $\Phi_{i}$ such that

$$
\phi_{i}-\phi_{j} \leq \ln \left(p^{i} x^{j} / p^{i} x^{i}\right) \quad(i \in I) .
$$

See Theorem 3.5, and also Afriat $(1972,1981)$, Diewert (1973) and Varian (1983).

Weak Separability: There exists a weakly separable function $f(x)=g\left(x_{1}, h\left(x_{2}\right)\right)$, such that $g\left(x_{1}, \eta\right)$ is strictly increasing in $\eta$, which satisfies the demand hypothesis.

Condition: There exist preorders $\succsim$ on $\left\{x^{i}\right\}_{\text {ier }}$ and $z^{\prime}$ on $\left\{x_{2}^{i}\right\}_{\text {iel }}$ such that

$$
\begin{aligned}
& p_{1}^{i} x_{1}^{j}+p_{2} x_{2}^{k} \leq p^{i} x^{i} \Rightarrow\left(x^{j} \approx x^{i} \text { or } x_{2}^{k}<x_{2}^{j}\right) \text { and } \\
&\left(x^{j} \prec x^{i} \text { or } x_{2}^{k} \leq x_{2}^{j}\right) \quad(i, j, k \in I), \\
& p_{1}^{i} x_{1}^{j}+p_{2}^{i} x_{2}^{k}<p^{i} x^{i} \Rightarrow x^{j}<x^{i} \text { or } x_{2}^{k} \prec^{i} x_{2}^{j} \quad(i, j, k \in I) .
\end{aligned}
$$

See Theorem 4.7 .

Concave Weak Separability: There exists a concave weakly separable function $f(x)=g\left(x_{1}, h\left(x_{2}\right)\right)$, such that $g$ and $h$ are concave and $g\left(x_{1}, \eta\right)$ is strictly increasing in $\eta$, which satisfies the demand hypothesis.

Condition: There exist numbers $u_{i}, v_{i}, \lambda_{i}>0$ and $\mu_{i}>0$ such that 


$$
\begin{aligned}
& u_{i} \leq u_{j}+\lambda_{j}\left[p_{1}^{j}\left(x_{1}^{i}-x_{1}^{j}\right)+\left(v_{i}-v_{j}\right) / \mu_{j}\right] \quad(i, j \in I), \\
& v_{i} \leq v_{j}+\mu_{j} p_{2}^{j}\left(x_{2}^{i}-x_{2}^{j}\right) \quad(i, j \in I) .
\end{aligned}
$$

See Diewert and Parkan (1978, 1985) and Varian (1983). Note that the theorem in Varian (1983) contains an omission. He does not require that the aggregator function $g$ and the subfunction $h$ are concave.

\section{G.2 Producer Demand}

In case of producer demand it is assumed that the data include the produced quantities $y_{i}$ for period $i$. Then the data ${ }_{1 s}$ of the form $\left\{\left(p^{i}, x^{i}, y_{i}\right)\right\}_{i e l}$. The list of necessary and sufficient conditions for the several hypotheses is as follows.

Producer Demand: There exists a function $f$ which satisfies the demand hypothesis and $f\left(x^{i}\right)=y_{i}$ for all $i \in I$, given the data $\left\{\left(p^{i}, x^{i}, y_{i}\right)\right\}_{i \in I}$.

Condition: One has

$$
p^{i} x^{j} \leq p^{i} x^{i} \Rightarrow y_{j} \leq y_{i}, \quad p^{i} x^{j}<p^{i} x^{i} \Rightarrow y_{j}<y_{i} \quad(i, j \in I) .
$$

See Theorem 8.1. See also Hanoch and Rothschildt (1972) and Varian (1984).

Techmical Progress: There exists an increasing series of functions $f_{i}, i \in I$, i.e. $f_{i} \geq f_{j}$ if $i \geq j$, such that each function $f_{i}$ satisfies the producer demand hypothesis for its corresponding data element $\left(p^{i}, x^{i}, y_{i}\right)$.

Condition: One has

$$
p^{i} x^{j} \leq p^{i} x^{i} \Rightarrow y_{j} \leq y_{i}, p^{i} x^{j}<p^{i} x^{i} \Rightarrow y_{j}<y_{i} \quad(j \leq i) .
$$

See Theorem 8.3 .

Hicks Neutral Change: There exists a function $f$ and numbers $t_{i}>0$, such that each function $t_{i} f$ satisfies the producer demand hypothesis for its corresponding data element $\left(p^{i}, x^{i}, y_{i}\right)$.

Condition: There exist values $\phi_{i}=\ln t_{i}$ such that

$$
\begin{array}{ll}
\phi_{i}-\phi_{j} \leq \ln \left(y_{i} / y_{j}\right) & \left(i, j \in I: p^{i} x^{j} \leq p^{i} x^{i}\right) \\
\phi_{i}-\phi_{j}<\ln \left(y_{i} / y_{j}\right) & \left(i, j \in I: p^{i} x^{j}<p^{i} x^{i}\right) .
\end{array}
$$

See Theorem 8.8 .

Neutral Change: There exists a function $f$ and numbers $t_{i}>0$, such that each function $f_{i}(x)=f\left(x_{1}, t_{1} x_{2}\right)$ satisfies the producer demand hypothesis for the corresponding data element $\left(p^{i}, x^{i}, y_{i}\right)$.

Condition: There exist values $\phi_{i}=\ln t_{i}$ such that

$$
\begin{array}{ll}
\phi_{i}-\phi_{j} \leq \ln \left(p_{2}^{i} x_{2}^{j} /\left[p^{i} x^{i}-p_{1}^{i} x_{1}^{j}\right]\right) & \left(i, j \in I: y_{j} \geq y_{i}, p^{i} x^{i}>p_{1}^{i} x_{1}^{j}\right), \\
\phi_{i}-\phi_{j}<\ln \left(p_{2}^{i} x_{2}^{j} /\left[p^{i} x^{i}-p_{1}^{i} x_{1}^{j}\right]\right) & \left(i, j \in I: y_{j}>y_{i}, p^{i} x^{i}>p_{1}^{i} x_{1}^{j}\right) .
\end{array}
$$

See Theorem 8.10 .

Linear Homogeneity: There exists a linearly homogeneous function $f$ which satisfies the producer demand hypothesis.

Condition: One has

$$
y_{j} \leq y_{i} p^{i} x^{j} / p^{i} x^{i} \quad(i, j \in I)
$$

See Theorem 9.1, and also Hanoch and Rothschildt (1972) and Afriat (1972).

Linear Homogeneity with Technical Progress: There exists an increasing series

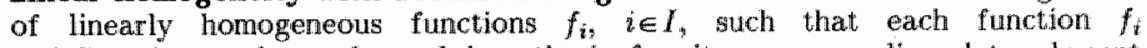
satisfies the producer demand hypothesis for its corresponding data element 
$\left(p^{i}, x^{i}, y_{i}\right)$

Condition: One has

$$
\left.y_{j} \leq y_{i} p^{i} x\right) / p^{i} x^{i} \quad(j \leq i)
$$

See Theorem 9.5 .

Linesur Homogeneity with Hicks Neutral Change: There exists a linearly homogeneous function $f$ and numbers $t_{i}>0, i \in I$, such that each function $t_{i}$ satisfies the producer demand hypothesis for its corresponding data element $\left(p^{i}, x^{i}, y_{i}\right)$

Condituon: There exist values $\phi_{i}=\ln t_{i}$ such that

$$
\phi_{i}-\phi_{j} \leq \ln \left(y_{j} p^{i} x^{i} / y_{i} p^{i} x^{j}\right) \quad(i, j \in I)
$$

See Theorem 9.9 .

Linear Homogeneity with Neutral Change: There exists a linearly homogeneous function $f$ and numbers $t_{i}>0, i \in I$, such that each function $f_{i}(x)=f\left(x_{1}, t_{i} x_{2}\right)$ satisfiles the producer demand hypothesis for its corresponding data element $\left(p^{i}, x^{i}, y_{i}\right)$.

Condition: There exist values $\phi_{i}=\ln t_{i}$ such that

$$
\phi_{i}-\phi_{j} \leq \ln \left(p_{2}^{i} x_{2}^{j} /\left[p^{i} x^{i} y_{j} / y_{i}-p_{1}^{i} x_{1}^{j}\right]\right) \quad\left(i_{j} j \in I: p^{i} x^{i} y_{j} / y_{i}>p_{1}^{i} x_{1}^{j}\right)
$$

See Theorem 9.12 .

Homotheticity: There exists a homothetic function $f$ which satisfies the producer demand hypothesis.

Condition: There exist numbers $\phi_{i}$ such that

$$
\begin{array}{ll}
\phi_{i}-\phi_{j} \leq \ln \left(p^{i} x^{j} / p^{i} x^{i}\right) & (i, j \in I) \\
\phi_{i}-\phi_{j} \leq 0 & \left(i, j \in I: y_{i} \leq y_{j}\right) \\
\phi_{i}-\phi_{j}<0 & \left(i, j \in I: y_{i}<y_{j}\right) .
\end{array}
$$

See Theorem 10.1, and also Hanoch and Rothschildt (1972) and Varian (1984). Note that both their theorems contain an omission. Hanoch and Rothschildt do not require

$$
\phi_{i}-\phi_{j}<0 \quad\left(i, j \in l: y_{i}<y_{j}\right)
$$

as is given in the condition above, and Varian does not require

$$
\phi_{i}-\phi_{j} \leq 0 \quad\left(i, j \in I: y_{i} \leq y_{j}\right)
$$

Weak Separabilty: There exists a weakly separable function $g\left(x_{1}, h\left(x_{2}\right)\right)$, such that $g\left(x_{1}, \eta\right)$ is strictly increasing in $\eta$, which satisties the producer demand bypothesiss.

Condition: There exists a preorder $\succsim$ on $\left\{x_{2}^{i}\right\}_{i \in I}$ such that

$$
\begin{array}{lll}
p_{1}^{i} x_{1}^{j}+p_{2}^{i} x_{2}^{k} \leq p^{i} x^{i}, y_{j}>y_{i} \Rightarrow x_{2}^{k}<x_{2}^{j} & (i, j, k \in I), \\
p_{1}^{i} x_{1}^{j}+p_{2}^{i} x_{2}^{k}<p^{i} x^{i}, y_{j} \geq y_{i} \Rightarrow x_{2}^{k}<x_{2}^{j} & (i, j, k \in I), \\
p_{1}^{i} x_{1}^{j}+p_{2}^{i} x_{2}^{k} \leq p^{i} x^{i}, y_{j} \geq y_{i} \Rightarrow x_{2}^{k} \leq x_{2}^{j} & (i, j, k \in I) .
\end{array}
$$

See Theorem 11.2.

Concave Weak Separability: There exists a concave weakly separable function $g\left(x_{1}, h\left(x_{2}\right)\right)$, such that $g$ and $h$ are concave and $g\left(x_{1}, \eta\right)$ is strictly increasing in $n$, which satisf tes the producer demand hypothesis.

Condition: There exist numbers $v_{i}, \lambda_{i}>0$ and $\mu_{i}>0$ such that 


$$
\begin{aligned}
& y_{i} \leq y_{j}+\lambda_{j}\left[p_{1}^{j}\left(x_{1}^{i}-x_{1}^{j}\right)+\left(v_{i}-v_{j}\right) / \mu_{j}\right] \quad(i, j \in I), \\
& y_{i} \leq v_{j}+\mu_{j} p_{2}^{j}\left(x_{2}^{i}-x_{2}^{j}\right) \quad(i, j \in I) .
\end{aligned}
$$

This is a simple modification of the conditions of Diewert and Parkan (1978, 1985), concerming concave weakly separable consumer demand and where output $y_{i}$ is not observed.

Weak Linearly Homogeneous Separability: There exists a weakly separable function $g\left(x_{1}, h\left(x_{2}\right)\right)$, such that $h$ is a linearly homogeneous and $g\left(x_{1}, \eta\right)$ is strictly increasing in $\eta$, which satisfies the producer demand hypothesis.

Condition: There exist values $\phi_{i}=\ln h\left(x_{2}^{i}\right)$ such that

$$
\begin{array}{ll}
\phi_{i}-\phi_{j} \leq \ln \left(p_{2}^{j} x_{2}^{i} / p_{2}^{j} x_{2}^{j}\right) \quad(i, j \in I), & \\
\phi_{i}-\phi_{j} \leq \ln \left(p_{2}^{i} x_{2}^{i} /\left[p^{i} x^{i}-p_{1}^{i} x_{1}^{j}\right]\right) & \left(i, j \in I: y_{j} \geq y_{i}, p^{i} x^{i}>p_{1}^{i} x_{1}^{j}\right), \\
\phi_{i}-\phi_{j}<\ln \left(p_{2}^{i} x_{2}^{i} /\left[p^{i} x^{i}-p_{1}^{i} x_{1}^{j}\right]\right) & \left(i, j \in I: y_{j}>y_{i}, p^{i} x^{i}>p_{1}^{i} x_{1}^{j}\right)
\end{array}
$$

See Theorem 11.4. Note that for price and quantity data we may assume that $g\left(x_{1}, \eta\right)$ is strictly increasing.

Linearly Homogeneous Weak Linearly Homogeneous Separability: There exists a linearly homogeneous weakly separable function $g\left(x_{1}, h\left(x_{2}\right)\right)$, such that the subfunction $h$ is linearly homogeneous and $g\left(x_{11}, \eta\right)$ is strictly increasing in $\eta$, which satisfies the producer demand hypothesis.

Condition: There exist values $\phi_{i}=\ln h\left(x_{2}^{i}\right)$ such that

$$
\begin{aligned}
& \phi_{i}-\phi_{j} \leq \ln \left(p_{2}^{j} x_{2}^{i} y_{j} / p_{2}^{j} x_{2}^{j} y_{i}\right) \quad(i, j \in I) \\
& \phi_{i}-\phi_{j} \leq \ln \left(p_{2}^{i} x_{2}^{i} / y_{i}\left[p^{i} x^{i} / y_{i}-p_{1}^{i} x_{1}^{j} / y_{j}\right]\right) \quad\left(i, j \in I: p^{i} x^{i} / y_{i}-p_{1}^{i} x_{1}^{j} / y_{j}>0\right)
\end{aligned}
$$

See Theorem 11.6. Note that for a finite price and quantity data set we may assume that $g\left(x_{1}, \eta\right)$ is strictly increasing.

Linearly Homogeneous Weak Separability: There exists a linearly homogeneous weakly separable function $g\left(x_{11}, h\left(x_{2}\right)\right)$, such that $g\left(x_{1}, 7\right)$ is strictly increasing in $\eta$, which satisfies the producer demand hypothesis.

Condition: There exists a preorder $\succsim$ on $\left\{v_{2}^{i}\right\}_{i \in I}$ such that

$$
\begin{array}{lll}
p_{1}^{i} w_{1}^{j}+p_{2}^{i} v_{2}^{k}<p^{i} v^{i} & \Rightarrow v_{2}^{k} \ll v_{2}^{j} & (i, j, k \in I), \\
p_{1}^{i} v_{1}^{j}+p_{2}^{i} v_{2}^{k} \leq p^{i} v^{i} \Rightarrow v_{2}^{k} \precsim v_{2}^{j} & (i, j, k \in I),
\end{array}
$$

where $w^{i}=y_{i}^{-1} x^{i}$ for all $i \in I$.

See Theorem 11.7. Note that this theorem proves only that the above is a necessary condition, and that for price and quantity data we may assume that $g\left(x_{1}, 7\right)$ is strictly increasing.

\section{G.3 Profit Maximization}

In case of profit maximization the data is also of the form $\left\{\left(p^{i}, x^{i}, y_{i}\right)\right\}_{i \in \text {. }}$.

Profit Maximization: There exists a function $f$ such that

$$
f\left(x^{i}\right)-p^{i} x^{i}=\max _{x} f(x)-p^{i} x_{,} f\left(x^{i}\right)=y_{i} \quad(i \in I) .
$$

Condition: One has

$$
y_{j} \leq y_{i}+p^{i} x^{j}-p^{i} x^{i} \quad(i, j \in l) .
$$

See Theorem 13.1, and also Hanoch and Rothschildt (1.972) and Varian (1984).

Technical Progress: There exists an increasing series of functions $f_{i}, i \in I$, such that each function $f_{i}$ satisfies the profit maximization hypothesis for 
its corresponding data element $\left(p^{i}, x^{i}, y_{i}\right)$.

Condition: One has

$$
y_{j} \leq y_{i}+p^{i} x^{j}-p^{i} x^{i} \quad(i, j \in I: j \leq i)
$$

See Theorem 13.8 .

Hicks Neutral Change: There exists anction $f$ and numbers $t_{i}>0$, such that each function $t_{i f}$ satisfies the profit maximization hypothesis for its corresponding data element $\left(p^{i}, x^{i}, y_{i}\right)$.

Condition: There exist values $\phi_{i}=\ln t_{i}$ such that

$$
\phi_{i}-\phi_{j} \leq \ln \left(\left[y_{i}+p^{i} x^{j}-p^{i} x^{i}\right] / y_{j}\right) \quad(i, j \in l) \text {. }
$$

See Theorem 13.10 .

Neutral Change: There exists a function $f$ and a series of numbers $t_{i}>0$, such that each function $f_{i}(x)=f\left(x_{1}, t_{i} x_{2}\right)$ satisfies the profit maximization hypothesis for its corresponding data element $\left(p^{i}, x^{i}, y_{i}\right)$.

Condition: There exist values $\phi_{i}=\ln t_{i}$ such that

$$
\phi_{i}-\phi_{j} \leq \ln \left(p_{2}^{i} x_{2}^{j} /\left[p^{i} x^{i}+y_{j}-y_{i}-p_{1}^{i} x_{1}^{j}\right]\right) \quad\left(i, j \in I: y_{j}-p_{1}^{i j} x_{1}^{j}>y_{i}-p^{i} x^{i}\right) .
$$

See Theorem 13.14.

Homotheticity: There exists a homothetic function $f$ which satisfies the profit maximization hypothesis.

Condition: There exist values $\phi_{i}$ such that

$$
\begin{aligned}
& \phi_{i}-\phi_{j} \leq \ln \left(p^{j} x^{i} / p^{j} x^{j}\right) \quad(i, j \in I), \\
& \phi_{i}-\phi_{j} \leq \ln \left(p^{i} x^{i} /\left[y_{j}-y_{i}+p^{i} x^{i}\right]\right) \quad\left(i, j ; y_{j}-y_{i}+p^{i} x^{i}>0\right) .
\end{aligned}
$$

See Theorem 14.1. In Varian (1984) weaker conditions are given concerning homothetic profit maximization, but the corresponding proof is incomplete.

Weak Separability: There exists a weakly separable function $g\left(x_{1}, h\left(x_{2}\right)\right)$, such that $g\left(x_{1}, \eta\right)$ is strictly increasing in $\eta$, which satisfies the profit maximization hypothesis.

Condition: There exists a preorder $\succsim$ on $\left\{x_{2}^{i}\right\}_{i \in I}$ such that

$$
\begin{array}{ll}
y_{j}-p_{1}^{i} x_{1}^{j}-p_{2}^{i} x_{2}^{k} \geq y_{i}-p^{i} x^{i} \Rightarrow x_{2}^{k} \precsim x_{2}^{j} & (k, i, j \in I) . \\
y_{j}-p_{1}^{i} x_{1}^{j}-p_{2}^{i} x_{2}^{k}>y_{i}-p^{i} x^{i} \Rightarrow x_{2}^{k} \prec x_{2}^{j} & (k, i, j \in I) .
\end{array}
$$

See Theorem 15.2. 


\section{REFERENCES}

Afriat, SN. (1967): The Construction of Utility Functions from Expenditure Data." International Economic Rewiew $7,67-77$.

(1973): "On a System of Inequalities in Demand Analysis: An Extension of the Classical Method," International Economic Review 14, $460-472$.

(1972): "Efficiency Estimation of Production Functions," International Economic Review 13, 568-598.

(1981): "On the Constructability of Consistent Price Indices Betwreen Several Periods Simultaneously," in Essays in the Theory and Measurement of Consumer Behaviour in Honour of Sir Richard Stone, ed. by A. Deaton. Cambridge: Cambridge Uniwersity Press.

Almon, C., L.M. Buckler, L.M. Horwitz and T. Reimbold (1974): 1985 Interindustry Forecasts of the American Fconomy, Lexington, Massachusetts: Lexington Books.

Antonelli, G.B. (1886): Sulla Teoria Mathematica Dell" Economica Potitica, Pisa: Tipografica del Folchetto. English translation in Preference, Utility and Demand, ed. by J.S. Chipman et al., New York: Harcourt, Brace Jovanovich, 1971, Chapter 16.

Berends-Ballast, H. (1987): "Lifetime of Capital Goods," Netherlands Official Statistics 2, 26-28.

Blackorby, C., D. Primont and R. Russell (1979): Duality, Separability, and Functional Structure: Theory and Economic Applications, Amsterdam: North-Holland.

Bronars, S.G. (1987): "The Power of Nonparametric Tests of Preference Maximization," Econometrica 55, 693-698.

Carre, B. (1979): Graphs and Networks, Oxford: Oxford University Press.

Central Planning Bureau (various issues): Centraal Economisch Plan, 's-Gravenhage: Staatsuitgeverij.

Chiappori, P., and J Rochet, (1987): "Revealed Preforences and Differentiabie Demand," Econometrica 55, 687-691.

Chipman, J.S., $\mathbb{L}_{*}$ Hurwicz and M.K. Richter, editors (1971): Preference, Utility and Demand, New York: Harcourt Brace Jovanovich.

De Nederlandsche Bank N.V. (1985): Monetaire tn financiele jaar-en kwartaalreeksen 1957-1983, Deventer: Kluwer.

Diewert, W.E. (1973): "Afriat and Revealed Preference Theory," Review of Economic Studies 40, $419-426$.

(1978): "Superlative Index Numbers and Consistency in Aggregation," Econometrica 46, $883-900$.

Diewert, W.E., and C. Parkan (1978): "Tests for the Consistency of Consumer Data and Nonparametric Index Numbers," Discussion Paper No. 78-27, University of Columbia.. 
Diewert, WE. and Parkan, C (1985): "Tests for the Consistency of Consumer Data," Journal of Econometrics 30, ne 1-2, 127-147.

Fare, R. and D. Primont (1995): Multi-Output Prodwction and Duality: Theory and Applications, Dordrecht: Kluwer Academic Publishers.

Fisher, F.M. (1971): "Aggregate Production Functions and the Explanation of Wages: A Simulation Experiment, The Review of Economics and Statistics 53, $305-325$.

Fisher, FM., RM. Solow and JM, Kearl 1977). "Aggregate Production Functions: Some CES Experiments," Review of Economic Studies 44, 305-320.

Floyd, R.W. (1962): "Algorithn 97: Shortesit Path" Communs Association Computing Machine 11, 493-497.

Gelauff, G.M.M., and B.H. Hasselman (1985): "Kapitaalkosten," Paper, Central Planning Bureau.

Gorman, W.M. (1953): "Community Preference Flelds," Econometrica 21, 53-56. $469-481$.

(1959): "Separable Utility and Aggregation," Econometrica 27,

Guardabassi, G. (1971): "A Note on Mimimal Essential Sets," IEE Transactions on Circuit Theory CT-18, $557-560$.

Hahn, F., and I. Schmoranz (1984): "Estimates of Capital Stock by Industries for Austria," The Review of Income and Wealth 30, 289-307.

Hanoch, G., and M. Rothschildt (1972): "Testing the Assumptions of Production Theory: A Nonparametric Approach," Journal of Political Economy 80, $256-275$.

Herstein, I.N., and J. Minnor (1953): "An Axiomatic Approach to Measurable Utility," Econometrica 211, $291-297$.

Houthakker, H.S. (1950): "Revealed Preference and the Utility Function," Economica 17, $159-174$.

Jorgenson, D.W. (1967): "The Theory of Investment Behavior" in Determinants of Investment Behavior, ed. by R. Ferber, New York.

Kailath, T. (1980): Linear Systems, Englewood Cliffs, N.J.: Prentice-Hall.

Koo, A.Y.C. (1963): "An Empirical Test of Revealled Preference Theory," Econometrica 31, 646-664. $39,89-98$.

(1971): "Revealed Preference - A Structural Analysis," Ecomometrica

Koo, A.Y.C. and G. Hasenkamp (1972): "Structure of Rewealed Preference - Some Preliminary Evidence," Journal of Polatical Economy 80, 724-744.

Koo, A.Y.C., and P. Schmidt (1974): "Cognitive Range in the Theory of Revealed Preference," Journal of Political Economy 82, 174-179.

Landsburg, S.E. (1981): "Taste Change in the United Kingdom, 1900-1955," Journal of Political Economy 89, 92-104.

Maks, JA.H. (J.978): "Consistency and Consumer Behaviour in the Netherlands, 1951-1977," European Ecomomic Review 11, 343-362.

(1980): Empirical Preference Orderings and Applied Demand Analysis, Dissertation, University of Groningen. 
(1982): "A Supplementary Method for Consumer Demand Analysis and Welfare Comparison Applied to United Kingdom and West German Data Sets:" Kwantitaticve Methoden 5, 56-77.

(1984): "Consumer Behaviour in the Netherlands, 1951-1977, A Non-parametric Approach," Memarandum of the Institute of Economic Research No. 159, University of Groningen, Presented at the 1984 European Meeting of the Econometric Society in Madrid.

Manser, E.M., and R.J. McDonald (1988): "An Analysis of Substitution Bias in Measuring Inflation, 1959-85," Econometrica 36, 909-930.

Mossin, A. (1972): "A Mean Demand Function and Individual Demand Functions Confronted with the Weak and Strong Axiom of Revealed Preference: An Empirical Test," Econometrica 40, 177-192.

Muellbauer, J. (1976): "Community Preferences and the Representative Consumer," Econometrica 44, $979-999$.

Netherlands Central Bureau of Statistics (1957): Investeringers in vastie activa, 1956 en 1957, Zeist: De Haan.

(1986): Bijdragen tot de statistioken : betreffende de
kapitaalgoederenvoorraad, s-Gnavenhage: Staatsuitgeverij.
(various issues): Statistics on Fixed Capital Formation in Industry,
's-Gravenhage: Staatsuitgeverij. Staatsuitgeverij.

(various issues): Nationale Rekiningen, 's-Gravenhage:

(various issues): Maandstatistiek van de Prijzen, 's-Gravenhage: Stratsuitgeverij.

- (various issues): Prijsanalyse, 's-Gravenhage: Staatsuitgeverij.

(various issues): Maandstatistiek wan de Binnenlandse Handel, 's-Gravenhage: Staatsuitgeverij.

- (various issues): Statistical Yearbook of the Netherlands, 's-Gravenhage: Staatsuitgeverij.

Netherlands Central Planning Bureau (various issues): Centraal Economisch Plan, 's-Gravenhage: Staatsuitgeverij.

Paccoud, $T$ (1983): "Stock of Fixed Assets in Industry in the Community Member States: Towards Greater Comparability," Studies of National Accownts No 2 , Luxembourg: Office for Official Publications of the European Communities.

Pontryagin, $\mathbb{L}_{\text {S.S., }}$ V.G. Boltyanskit, R.V. Gamkrelidze and E.F Mischenko 1962): The Mathematical Theory of Optimal Processes, translated by Tirogoff, New York: Interscience.

Richter, M.K. (1966): "Revealed Preference Theory," Econometrica 34, 635-645. $131-181$.

Rockafellar, R.T. (1970): Convex Anclysis, Princeton: University Press.

Roubens, M., and P. Vincke (1985): Preference Modeling, Berlin: SpringerVerlag.

Sagan, H. (1969): Introduction to the Calculus of Variations, New York: McGraw-Hill. 
Samulson, P.A. (1948): "Consumption Theory in Termis of Revealed Preference," Economitea $15,243-253$. $17,355-385$. (1950): "The Problem of Integrability in Utility Theory" Economica

Shephard, RW. (1953): Cost and Production Functions, New Jersey: Princeton. Princeton. (1970): The Theory of Cost and Production Functions, New Jersey:

Stahmer, C. (1983): "Alteraufbau des Anlagwermogens nach Wirtschaftsbereichen," Wintschaft und Statistrek 4, Statistisches Bundesamt, 285-290.

Szpilrajn, E: (1930): "Sur J'Extension de lOrdre Partial," Fundamenta Mathematúcae 16, $386-389$.

Takayama, A. (1974): Mathematical Economics, Hinsdale, Illinois: The Dryden Press.

Uzawa, H. (1960): "Preference and Rational Choice in the Theory of Consumption," pp. 129-148 in Mathematical Methods in the Social Sctiences, ed. by K.J. Arrow, S. Karlin and P.C. Suppes, Stanford, California: Stanford University Press.

Varian, H.R. (1982): "The Nonparametric Approach to Consumption Analysis," Econometrica $50,945-973$.

(1983): "Non-parametric Tests for Consumer Behaviour," Review of Economic Studies 50, 99-110.

(1984): "The Nonparametric Approach to Production Aralysis," Econometrica 52, $579-597$.

(1988): "Revealed Preference with a Subset of Goods," Jourral of Economic Theory 46, 179-185.

Vartia, Y.O. (1978): "Fisher"'s Five Tines Fork and Other Quantum Theories of Index Numbers," in Theory and Applications of Economic Indices, ed. by W. Eichhorn et al., Würzburg.

Vermeend, W.A.T.G. (1983): Fiscale investeringsfaciliteiten: een onderzoek naar de werking van vervroegde afschrijving, whesteringsaftrek en de wet investeringsrekening, Dissertation, Arnhem: Gouda Quint.

Warshall, S. (1962): "A. Theorem on Boolean Matrices," Journal of the American Association for Computing Machinery 9, 11-12.

Zemanian, A.H. (1965): Distribution Theory and Transform Analysis, New York. 


\section{RELATED LITERATURE}

Adamowicz, W.L., and T. Grahamtomasi (1991): Revealed Preference Tests of Nonmarket Goods Valuation Methods," Journal of Environmental Econonics and Management 20, nr $1,29-45$.

Afriat, S.N. (1977): The Price Index, London: Cambridge University Press. University Press.

Aizcorbe, A.M. (1991): "A Lower Bound for the Power of Nonparametric-Tests" Journal of Business and Economic Statistics 9, ni $4,463-467$.

Ashenfelter, $0 .$, and D. Sullivan (1987): "Nonparametric-Tests of Market-Structure - An Application to the Cigarette Industry," Jowrnal of Industrial Economics 35, nr 4, 483-498.

Banker, R.D., R.F. Conrad and R.P. Strauss (1986): "A Comparative Application of Data Envelopment Analysis and Translog Methods - An Illustrative Study of Hospital Production," Management Science 32, nr 1, 30-44.

Banker, R.D., and A. Maindiratta (1988): "Nonparametric Analysis of Technical and Allocative Efficiencies in Production," Ecomometrica 56, ni 6 , $1315-1332$.

Barnhart, S.W., and G.A. Whitney (1988): "Nonparametric Analysis in Parametric-Estimation - An Application to Translog Demand Systems," Review of Economics and Statistics 70, $\mathrm{nr} 1,149-153$.

Barshira, Z. (1992): "Nonparametric Test of the Expected Utility Hypothesis," American Journal of Agricultural Economics 74, $\mathrm{nr} 3,523-533$.

Basu, K. (1984): "Fuzzy Revealed Preference Theory," Jowrnal of Economic Theory 32, 212-217

Bronars, S.G. (1987): "The Power of Nonparametric-Tests of Preference Maximization," Econometrica 55 , nr 3, 693-698.

Browning, M. (1989): "A Nonparametric Test of the Life-Cycle Rational-Expectations Hypothesis," International Economic Review 30 , nr 4 $979-992$.

Burton, M.P., and T. Young (1991): "Nonparametric-Tests for Changes in Consumer Preferences for Meat in Great-Britain," Journal of Agricultural Ecoriomics 42, nr 2, $138-145$.

Carey, M. (1989): "Estimating Nonparametric Convex-Functions Using Gradient Data," European Journal of Operational Research 41, nr 1, 73-85.

Chang, K.P., and Y.Y. Guh (1.991): "Linear Production-Functions and the Data Envelopment Analysis," European Journal of Operational Research 52 , $\mathrm{nr}$ 2, $215-223$.

Charnes, A., W.W. Cooper and R.M. Thrall (1986): "Classifying and Characterizing Efficiencies and Inefficiencies in Data Development Analysis," Operations Research Letters 5, nr 3, 105-110.

Chavas, J.P., and T.L. Cox (1988): "A Nonparametric Analysis of Agricultural Technology," American Journal of Agricultural Economics 70, nr 2, 303-310. 
Chavas, J.P., and T.L. Cox (1990): "A. Nonparametric Analysis of Productivity - The Case of Unted-States and Japanese Manufacturing, American Economic Revuev 80, nir 3,450-464.

Chavas, J.P. and Cox, T.L. (1992): "A Nonparametric Analysis of the Influence of Research on Agricultural Productivity," American Journal of Agriculiural Economics 74, nir 3, 583-591.

Chavas, J.P., and T.L. Cox (1993): "On Generalized Revealed Preference Analysis,"Quarterly Jowrnal of Economics 108, nr 2, 493-506.

Chiappori, P.A. (1988): "Rational Household Labor Supply," Econometrica 56, nr $1,63-90$.

(1990): "On Falsifiability of Consumer Theory," Revue Economique 41, nr $6,1001-1026$.

Clark, S.A. (1988)" "Revealed Independence and Quasi-Linear Choice," Oxford Economic Papers-New Series 40, nr 3,550-559.

Epstein, L.C., and A.J. Yatchew (1985): "Nonparametric Hypothesis-Testing Procedures and Applications to Demand Analysis," Journal of Ecomometrics 30, ni $1-2,149-169$.

Färe, R., and S. Grosskopf (1985): "A Nonparametric Cost Approach to Scale Efficiency," Scandinavian Journal of Economics 87, nr 4, 594-604.

Färe, R., S. Grosskopf and C.A.L. Lovell (1987): "Nonparametric Disposability Tests," Journal of Economies-Zeitschrift fur Nationalokonomie 47, nr 1 , $77-85$.

Färe, R., S. Grosskopf and H. Lee. (1990): "A Nonparametric Approach to Expenditure-Constrained Profit Maximization," American Journal of Agricultural Economics $72, \mathrm{nT} 3,574-581$.

Färe, R., S. Grosskopf and S.K. Li (1992): "Linear-Programming Models for Firm and Industry Performance," Scandinavian Journal of Economics 94, nr 4, $599-608$.

Farrell, M.I. (1957): "The Measurement of Productive Efficiency", Journal of the Royal Statistical Society, Series A, CXX Part II, 253-290.

Fawson, $C$, and C.R. Shumway (1988): "A Nonparametric Investigation of Agricultural Production Behavior for United-States Subregions," American Journal of Agricultural Economics 70, ne 2, 311-317.

first, $2 .$, S.T. Hackman and U. Passy (1993): "Efficiency Estimation and Duality-Theory for" Nonconvex Technologies," Journal of Mathematical Economics 22, nr 3, 295-307.

Fisher, $D_{1}$, and M. Mccrickard (1992): "Rational-Expectations and the Demand for Money - A Nonparametric Approach," Journal of Macroeconomics 14, nr 4, $573-591$

Fuchsseliger, S., and A. Pfingsten (1986): "Cost-of-Living Indexes Based on Demand-Functions," Joumal of Economics-Zeitschrift fur Nationalekonomie: 46, nr $1,49-64$.

Green, E.J., and K. Osband (1991): "A Revealed Preference Theory for Expected Utility," Review of Economic Studies 58, nr 4, 677-695.

Gross, J. (1991): "On Expenditure Indexes in Revealed Preference Tests," Jowrnal of Polatical Economy 90, ar 2, 416-419. 
Garbaccio, R.F., B.E. Hermalin and N.E. Wallace (1992): "Nonparanetric Methods to Measure Efficiency: A Comparison of Methods," UC Berkeley nr $92-206$.

Houtman, M., and J.A.H. Maks (1983): "On Mimimall Feedback Node Sets in Revealed Preference Theory," Memorandum of the Instutute of Economic Research No. 136, University of Groningen.

Houtman, M., and J.A.H. Maks (1983): "Maximal Homothetic Subsets in a Given Data Set, Nemorandum of the Institute of Economic Research No, 137, University of Groningen.

Houtman, M., and J.A.H. Maks (1984): The Existence of Homothetic Utility Functions Generating Dutch Consumer Datia," Memorandum of the Institute of Economic Research No. 158, University of Groningen, Presented at the 1984 European Meeting of the Econometric Society in Madrid.

Houtman, M. (1986): "Nonparametric Tests for Weak Separability," Presented at the 1986 European Meeting of the Econometric Society in Budapest, Hongary.

Jerison, D., and M. Jerison (1992): "Approximately Rational Consumer Demand and Ville Cycles," Journal of Economic Theory 56, nr 1, 1.00-120.

Knoblauch, V. (1992): "A Tight Upper Bound on the Money Metric Utillty Function," American Economic Review 82, nr 3, 660-663.

$41-45$

(1993): "Recovering Homothetic Preferences," Economic Letters 43,

Lewbel, A. (1991): "The Rank of Demand Systems - Theory and Nonparametric-Estimation," Econometrica 59, nr 3, 711-730.

Matzkin, R.L. (1991): "Axioms of Revealed Preference for Nonlinear Cholce Sets," Econometrica 59, nr 6, $1779-1786$.

Matzkin, R.L., and M.K. Richter (1991): "Testing Strictly Concave Rationality," Jowrnal of Economic Theory 53, nr 2, 287-303.

Moschini, G. (1990): "Nonparametric and Semiparametric Estimation - An Analysis of Multiproduct Returns to Scale," American Joumal of Agricultural Economics 72, nr 3, 589-596.

Orr, B., and K.J. Lee (1990): "A Nonparametric Analysis of Variable Cost Minimization in the United-States Paper-Industry," Applied Economics 22, nr $6,839-852$.

Patterson, K.D. (1991): "A Nonparametric Analysis of Personal Sector Decisions on Consumption, Liquid Assets and Leisure," Economic Journal 101, nr 408 , $1103-1116$.

Peters, H., and P. Wakker (1991): "Independence of Irrelevant Alternatives and Revealed Group Preferences," Econometrica 59, nr 6, 1787-1801.

Samuelson, $\mathbb{L}$. (1987): "A Test of the Revealed-Preference Phenomenon in Congressional Elections," Public Choice 54, nr 2, 141-169.

Seiford, L.M., and R.M. Thrall (1990): "Recent Developments in Dea - The Mathematical-Programming Approach to Frontier Analysis," Journal of Econometrics 46, nr 1-2, $7-38$.

Sengupta, J.K. (1989): "Nonparametric-Tests of Efficiency of Portiolio Investment," Journal of Economics-Zeitschrift fur Nationalekonomie so, nr $1,1-15$. 
Swofford, J.L., and G.A. Whiney (1987): Nomparametric-Tests of Utility Maximization and Weak Separability for Consumption, Leisure and Money," Review of Ecomomics and Statistics 69, nr 3,458-464.

Swofford, IL., and GA. Whitney (1988): A Comparison of Nonparametric-Tests of Weak Separability for Anmual and Quarterly Data on Consumption, Leisure, and Money," Journal of Business and Economic Statistics 6, nr 2, $241-246$.

Swofford, J.Li, and G.A. Whitney (1990): Bounding an Economic Monetary Aggregate Under Nonhomothetic Preferences," Joumal of Business and Economic Statistics 8, nx 1, $137-141$.

Swofford, J.L.; and A. Whitney (1994): "A Revealed Preference Test for Weakly Separable Utility Maximization with Incomplete Adjustment;" Journal of Econometrics $60,235-249$

Tsur, Y. (1989): "On Testing for Revealed Preference Condutions," Economics Letters 31, ni 4, 359-362.

Ullah. A. (1988): "Nonparametric-Estimation of Econometric Functionals," Canadian Journal of Economics-Revue Canadienne d'Economique 21, nr" 3, $625-658$.

Valdmanis, V. (1992): "Sensitivity Analysis for Dea Models - An Empirical Example Using Public ws Nfp Hospitals," Journal of Public Economics 48, nr $2,185-205$.

Varian, H.R. (1983): "Nonparametric-Tests of Models of Investor Behavior," Journal of Financial and Quantitative Analysis 18, nr 3, 269-278.

- (1985): "Nonparametric Analysis of Optimizing Behavior with Measurement Error," Journal of Econometrics 30, $\mathrm{nr} 1-2,445-458$.

(1988): "Estimating Risk-Aversion from Arrow-Debreu Portfolio Choice," Econometrica 56, nr 4, 973-979.

Econometrics 46, nr 1-2, 125-140.

Yatchew, A.J. (1985): "A Note on Non-Parametric Tests of Consumer-Behavior," Economics Letters 18, nr 1, 45-48.

(1992): "Nonparametric Regression Tests Based on Least-Squares," Econometric Theory 8 , nr 4, 435-451. 
Emboldened page numbers indicate an entry in a definition

absorption of a vertex 171,178 absorptive matrix or labelled graph 26, 27, 33, 118, 175, $176-178$

active constraints 38,149

acyclic graph $170,171,173,175$

adjacency mitrix $35,37, \mathbf{1 6 9}$, $170,174,175-177$

adjoint operator $\mathbf{x i v}, 142$

adjustment costs 141

Afriat, S.N. $2,7,9,13,21,24$, $31,34,188,189$

aggregate data $7,18-19,58-65$,

$162,181-1.83$

aggregator function $42,43-45$,

$47,104,106,110,114,132$,

134,189

algorithm

absorptive test 178

bisection 15

minimal feedback sets 171,179

shortest path, transitive closure 175

Almon, C. 157

analytic function 180

angular momentum 1 .

antisymmetric binary relation $\mathbf{1 6 5}$,

166,174

Antonelli, G.B. 7, 11

approximately finite data $51,52-54$, 57

approximation

gamma distribution 159

user cost of capital $153-154$

arcs $169,170,176$

arithmetic price index $60,61-63$

assets $137,152,155$

asymmetric relation 165

axiom of revealed preference 7,9 ,

$11,15-16,17,18-21,47,49$,

$51-52,57-59,63,69-70,73,90$, 169

base period $35,60,61-62$

belief in consumer behaviour 65 , 162

Berends-Ballast, H. 157

bias of price indices 65

binary relation $x i i i, 10,15,17$,
$20,165,166-167,169-170$,

$173-175,177$

bisection $15,31,70,77,85$

Blackorby, C. 42

Boolean matrix and graph $\mathbf{1 6 9 - 1 7 0}$, $173-175,177-178$

Boolean patth algebra 174,177

bounded function 54,56

bounds xili, $7,15,24,29,31-40$, $68,70-80,84-97,101-103,106$, $109-110,116-127,131,163-164$, $185-186$

branch-and-bound algorithm 171, 179

branches of industry 181, 183

Bronars, S.G. 63

budget $2,4-5,10,13,19,58$, $62-63,65,112,163$

buildings 181,183

capital 1-3, 67-68, 79-80, 95-98, 125-127

capital stock $2,3,136,138-142$, $144,147-149,156-157,159,16 \%$, 181-183

Carre, B. 173-175

CES-function 58

chained index $36,39,60,61-63$, 65,183

Chiappori, $P .163$

Chipman, J.S. 9, 11

closed mapping $49,50,52,54-56$, 71,78

closure of a relation or graph xiit, $16-17,19,33,95,125,165,175$

Cobb-Douglas function 58

coefficient of technical elficiency 120,121

commodities $7,28,34,58-64$, $181-182$

compact set $48-49,53-54$

competitive

consumer 6

market for output 113

complement xili, 17,165

complete relation $\mathbf{1 6 5}$

computer program $8,47,100$

concave function $7,22-23,27,29$, $39-42,45,52,56-57,103,105$, $131,133,135,163,188-191$ 
cone $24,25-41,84-110,116,124$, 129,131

connected component 170,172

constraint $2,4,38,58,141,148$, 163

conserved properties $1,6,18,25$, $28,34,45,47,164$

conisumer demand $1-2,4-9,10$, $11-65,69,71,101,104,117-118$, $162,188,191$

contagious erlang distribution 157 , $160-161,182$

continuous function $22-23,27,29$, $45,48,51,53-57,105-106,117$, 133,139

continuous-time model 161

convergence $49,55-56$

convolution xili, $139,150,163$, 180

cost function $2,6,9,10-135$

cost minimizing behaviour $\theta, 10$, $49-50,55$

coefficients of

technical efficiency 69

neutral change 79

countable data set 90,121

counter example 9, 50-51.

cover 51-53

current period 35,60

cycle path $26,170,171,175-178$

data enclosure 12

De Nederlandsche Bank N.V. 184

decay of investments $2,140,142$,

$147,163,184$,

delta distribution $\mathbf{x i v}, \mathbf{1 3 7}, 147$

Diewert W.E. 7, 24, 31, 42, 59,

$63,65,135,188-189,191$

difference

upper bound xiv, 117

lower bound xiv, 117

differentiability 163

differential equations 11

digraph 170

direct index 60,62

directed graph 170

discontinuity $22-23,29,53,180$

discounted cost $136,140-141$,

$1.48-155, \quad 183-184$

discrete-time model 161,182

distribution theory 140,143

diverging bounds 123,126

dual

approach 44

relation 12

theorem 54

Dutch industry data $66-135,181$,
$182-187$

dynamic multiplier 147,149

economical inefficiency 2,6

efficiency

level $13-15,31,35-36,38$, $51-57,61-65,77,80,82,85$, $120,123,125,126,185-186$

transformation $2,9,12,13-14$, $29-30,79-82,114$

upper bound $15,29,31,35,68$, $70-74,77,79-82,85,87-93$, $95-96,98,101-102,106,116$, $118,120,122-123,125,127$

efficient behaviour $6,9,13-15$, $24,27,30-31,36,38,64-65$, $116,122,162$

elementary particles 164

energy 1, 18, 164

equipment 181,183

equivalence relation $18,105,166$, 170

Erlang distribution $157,158-159$, 182

essential vertex $170,171,178,179$

exponential

decay $2,142,147,163,184$

discounted price 136,140 ,

$150-155,184$

surwival function 137,138 , $141-143,149,152,155,157$

retirement distribution 137,185

factors

Hicks neutral change 75

Hicks neutral progress 122

neutral progress capital and labour $80,1.27$

Fàne, $R$. 115

feedback vertex set 169, 171, 172 ,

178,179

fictitious economies 58

finite-dimensional matrix 176-177

Fisher, F.M. 58

Fisher price index $60,61-63,65$

Floyd, R.W. 175

forecast of economic behaviour 163

frontier production function xiv,

$2,66-67,68-70,73,81-82$,

$86-87,89-90,114,119,121$

Fubini's theorem 139,145

gamma distribution 137, 151-153,

$155,157,159,161,163,182-185$

GARP 182-187

gauge symmetry 10

Gelauff, G.M.M. 184 
geometric index $7,60,61-63$

global analysis $7,11-12$

Gorman, W.M. 58

graph $169,170-1179$

gross investment flow 139

Guardabassi, G. 171

Hahn, F. 157

Hanoch, G. 84, 100, 188-191

Harrod neutral change $\mathbf{6 7}$

Hasenkamp, G. 58

Hasselman, B.H. 184

Herstein, I.N. xiii, 165

Hicks neutral

change $67,74-75,78,89$, $91-92,121-123,186$, $189-190,192$

progress $67,68,74,76-77$, $89,93,122-123,131,187$

homothetic function $1,24,31,61$, $58-59,63-65,68,99,101-102$, $108,114,129-132,162,187$, 190,192

Houthakker, H.S. 7, 11

hyperplanes 7

incident arc 170

inconsistent behaviour 58-59, $64-65$

individual demand $58-59$

induced order $10,15,46,105,134$

industry data 181-182

inefficiency $2,6,28,66,118$

inefficient behaviour $14,21-22$,

$72,91,121,162$

infinitely differentiable utility

function 163

inflation $184-185$

innerproduct xiv, 141, 142

input-output $58,68,181-182$

inputs $66-67,78-79,112-113,136$, $181-182$

Integrability Problem 7, 11

intersection 49,52

invariance 83

invariant $1,5-6,9-10,24,28$, $44-45,108-109,145$

irreflexive $165,167-168,177$

isoquant $27,83-84$

join operation 173

Jorgenson, D.W. 141

Kailath, T. 156, 157

Kearl, J.H. 58

Koo, A.Y.C. 58, 59 labelling 174

labelled giaph $35,173,174$, $175-179$

labour $67,79,80,95-98,125-127$, $182,186-187$

Lagrange multiplier 115,148

Lagrangian $1,18,28,24-25,43$, $115,163-164$

Landsburg, S.E. 18, 59

Laplace transform xiil, 143 , $149-150,153,156-157,159,180$

Laspeyres price index $35-36,39$, $54,60,61-63$

length or cost of a path 175

lifetime of investments 3 , $136-137,145,147,152$, $154-155,161,182-183$

linear:

liomogeneity $1,5,7,24$,

$25-41,45,64-65,68$, $78-81,83-97,99-102$, $104,106-111,114-119$ $129-131,162-163,186-191$

operator $139,140-145$; $148-149$

order 165

oriented graph 170

local analysis $7,11,42$

locally nonsatiated function 5 , $48,54,56-57$

logistic probability distribution 137

long-term interest $183-184$

loop $\mathbf{1 7 0}$

lower bound xiv, 31-34, 37, 41,

$70,77,80,87,117,126$

lower bound $L$ xiv, 70

lower semicontinuous function $\mathbf{5 2}$, $54,56-57$

macro clatia $58-59$

Maks, J.A.H. 18, 59

Manser, E.M. 31, 59, 63

marginal

cost of capital 151

reventue of capital 148

monopolistic market 113

maximum principle $2,141,147,149$

McDonold, R.J. 31, 59,63

measure theory 145

mechanics $1,18,28,145$

micro data, 58-59

Milnor, $J$, xili, $\mathbf{1 6 5}$

minimal feedback vertex set 169,171

mode 153

moment 153

monotonic function 101-103, 131 
monotonic transformation $10,50-52$, $68,71-72,74,99,101-102,104$ $106,114,129,131$ monotonically uncreasing function $22,27,45,52,104-105,133$

Mossin, A. 58,59

Muellibutuer, $J .58$

multiple outputs 117,163

myopic producer 149

narrow bounds 80,95

neoclassical theory $2,4,6-7,11$, 24,112

Netherlands Central Burean of

Statistics $7,61-63,68,137$,

$141,151-152,162,182$

Netherlands Central Planining

Burean 68, 181-182, 184

Neumann series 144

neutral

change $67,78-79,81,94-98$, $124-127,185$

progres $67,79-80,97-98$, $127-128,186$

node 170

Noether theorem 1

noncompetitive market 2,6

nonlinear budget constraint 2

nonlinear investment costs 147 , 149

non-aggregate data 62

nonsatiated function $5-6,48,54$, 56-57

normal probability distribution 137

operator xili-xiv, 139-145

ordering $18,70,101$

orders $1,10-11,15,46-47,49$,

166

ontlying observation 96

Paasche price index $36,39,60$, $61-63,181,183$

Pidcoud, T. 157

panel data 58

parametric approach $3,5,7,24$, $31,58,137,140,152,154,157$, $162-163$

Parkan, C. 7, 31, 42, 59, 63, 135, 189,191

partial efficient behaviour 13

partial order $47,165,166-167,174$

path algebra 173, 174-178

patths xiti, $35-38,169,170,173$,

$175-176$

perpetual inventory method 139, 156
Poincare transfomation 1

pointwise limit function 41,50 ,

$52,55-56,71,87$

polynomes 157

Ponitryagin, L.S. 2, 147, 149

pre-war data 59

predecessor 170

preference $69-71,73-75,82,90,134$

preference order $1,5,6,9-12,15$;

49

preorder xiif, $5,10,11-12$,

$15-16,45-47,49,105,110,133$,

$165,166,167,169,173,188$,

190-192

probability 1, 64, 137, 140, 151,

$153-154,157,183,162$

present value of profit 136

producer demand with observed

output 66

profit maximizing behaviour

112-113, 114-135, 191-192

with observed output 114

Primont, D. 115

quantum theory $1,2,145$

quasi order 165

quasi-concave function 105,133

random data $7,58,62-64,162$

rationalizing

preorder and utility function 12

production function $\mathbf{6 7 - 6 8}$

rays $25,28,34,38,83$

recession 37

rectangular survival function 137 ,

$138,141,145,147,151-153,155$

reflexive binary relation $10, \mathbf{1 6 5}$, 174

regression 89,120

relative

lower bound xiv, 31-32

upper bound $x i v, 32,34,85$, $101-102,110,117$

retired investments 1,39

retirement distribution 3,136 ,

$137,139-140,144-145,149$,

$151-157,159-161,163,182-185$

Richter, M.K. 2, 12

risk premium 184

Rochet, J. 163

Rockafellar, R.T. 52, 56

Rothschildt, M. 84, 100, 188-191

Roubens, M. 166

Santuelson, P.A. 7, 11

satiation 51

scaled data $78,83-84,86,108-111$ 
Schmidi, P. 59

Schmoranz, I. 157

seasonal influences 59

self - wulfilling prophecy 65,162

separating hyperplanes 7

shadow cost of capital 141

shape of survival function 152 ,

$154-156$

Shephard, R.W. 99

shortest path xiii, $35-38,75,77$,

$100,173-178$

shortest path algebra 174, 175-178

simulation $156,160-161$

single-output 66,163

Solow, R.M. 58

Solow neutral change 67

step function xiv

stochastic elements 59

Strahmer, C. 157

state-space realization $\mathbf{1 5 6}, \mathbf{1 5 7}$,

$159-160,182$

statistical theory 156

strict total order 165

strongly

complete binary relation 10 , 165

conmected component 170,172

connected grap 170

subfunction $42,45,48,68$,

$104-108,110,114,132,189,191$.

subgraph 170,175

subsidies $181-182$

successor 170

superposition principle 64-65

survival function $3,136-137$,

$138-160$

symmetric relation 165,166

symmetry $1,5,6,9-10,18,24-25$,

$28,34,42-45,48-49,129,132$,

$145,153-154,162-164$

Szpilrajn, E. 166

Szapilrajn's theorem 166

Takayama, A. 141

taste change 59

tax 181-182, 184-185

technical

progres xiv, 66, 67, 68, $72-73,81,85,88-91,114$, $118,120-121,162,189,191$, 186

change 67, 69

efficiency $68-74,88-91$, 120-121, 163

efficiency upper bound $\mathbf{7 0}$, $71,73,68,88-91$

textbook theory $1-2,9,11,66$,
$112-113,142$

thick" indifference curve 4-5

time-independent 18,149

Törnquist price index $60,61-63,65$

total preorder 165

transitive

closure xiii, 16-17, 165, 175

binary relation 10,165

triangular matrix $18-19, \quad 31$

unit-isoquant 83-84

unobserved output $75,99,101$,

$104,117,188$

unrelíable data $2,8,64$

upper

bound xiv, $7,15,24,29-40$, $68-98,101-102,106$, $109-110,116-127,186$

bound of xiv, 85

semicontinuous function 27 ,

$52,54,56-57$

upward bias 59,65

user cost of capital $2,3,136$,

$140-144,150-154,163,183-185$

utility maximizing behaviour $\mathbf{9 - 1 0}$, $49-50,55,58-59$

Uzawa, H. 7, 11

Varian, H.R. $7,17-19,21,24,30$ $42,59,84,90,99-100,131$, $134-135,188-192$

variance $151-159,161,182-183$

Vartia, Y.O. 65

Vermeend, W.A.T.G. 184

vertex $37-38,169,170-171$,

$174-179$

Vincke, P. 166

Warshall, S. 165, 175

waste $4,13,70$

weak

closure $33,46,47,95,105$, $125,134,175$

order $10,17,20-21,165$, $166-167,178$

separability $1,5,7,42$, $43-48,68,104-111,114$, $129,132-135,162-163$, $187-188,190-192$

Weibull probability distribution 137 weight period $60,61-62$

Zemanian, A.H. 140,143 


\section{NEDERLANDSE SAMENVATTING / SUMMARY IN DUTCH}

Het onderwerp "Niet-parametrische consumenten- en producentenanalyse" is verwant actu de niet-parametrische statistiek. Deze statistiek is nivet gebaseerd op parametrische specificaties van kansverdelingen, maar gaat ervan wit dat de kansverdeling lid is van een niet-parametrische familie van kansverdelingen. Zo'n familie wordt vastgelegd door symmetrie-eigenschappen voor de knswerdeling te specificeren.

De analogie var deze benadering in de economische wetenschappen is het gebruik van een niet-parametrische specificatie voor de nuts- of produktiefunctie. Voor datat van economisch gedrag kan gepostuleerd worden dat deze gegenereerd is door middel van een functie, die an een niet-parametrische specificatie voldoet. Door toetsen te ontwikkelen, om na te gaan of aan zo'n hypothese is voldaan, kan men de bruikbarheid toetsen van annames betreffende economisch gedrag en de vorm van de nuts- of produktiefunctie. Deze niet-parametrische toetsen kumnen hulp bieden bij het kiezen van een parametrische specificatie van economisch gedrag.

Dit proefschrift beschrijft een groot aantal van deze niet-parametrische toetsen. Het gaat hierbij om econornisch gedrag van nutsmaximering, kostenminimering en winstmaximering, watrbif de volgende niet-parametrische specificaties beschouwd worden: lineaire homogeniteit, homotheticiteit, scheidbaarheid en technische vooruitgang. De toetsen zijn toegepast op prijzen en hoeveelheden, betreffende consumenten- en producentengedrag in Nederland. Zo wordt een indruk gegeven van de mogelijkheden die deze theorie biedt. Bovendien is als experiment een aantal miet-parametrische toetsen voor consumentengedrag toegepast op geaggregeerde random data. De random data werden voor dit doel geaggregeerd met aggegegratie methodes, die het Centraal Bureau voor de Statistiek voor de Nederlandse consumentengegevens heeft gebruikt.

Uit de resultaten van de niet-parametrische toetsen valt in het algemeen niet op tie maken welke specificatie gekozen moet worden. De toetsen geven wel aan of specificaties in strijd zijn met de data. Zo wordt duidelijk welke specificaties zeker niet gebruikt moeten worden. Een duidelijk voorbeeld hieryoor leveren de resultaten van de niet-parametrische toetsen voor de gegevens van de Nederlandse industrie. Deze geven aan dat winstmaximering, zonder gebruik te maken van de aanname var technische vooruitgang, niet in aanmerking komt alls gedrags-specificatie. Winstmaximering met technische vooruitgang is wel een toegestane gedrags-specificatie. Een andere mogelijkheid, die niet uitgaat van technische vooruitgang, is de weronderstelling van kostenminimerend gedrag.

Uit de resultaten blijkt dat yoorzichtigheid is geboden bij het trekken van conclusies over geaggregeerde consumentengegevens. Gebleken is namelijk dat geaggregeerde random data voldeden aan de hypothese van consumentengedrag. De oorzak hiervan is het gebruik van een voor de hand liggende regel betreffende consumentengedrag: prijs maal hoeveelheid is gelijk aan de uitgaven. Prijsindices en bijbehorende hoeveelheden krijgen daardoor de neiging on zich in tegengestelde richting te bewegen, datgene wat men van het gedrag van een consument zou verwachten. Het geloof in consumentengedrag werkt hier dus als een self-fulfilling prophecy.

In de praktijk blijken de niet-parametrische toetsen - op de "revealed preference" toets na - de gestelde hypotheses over efficient economisch 
gedrag te verwerpen: Er worden twee maniecen arangegeven waarmee bepaald kan worden in welke mate zo'n hypothese verworpen wordt. Ten eerste kan men zo weinig mogelijk waarnemingen uit de dataverzameling verwjjeren, zodanig dat de overblifwende gegevens voldoen an de hypothese. De tweede methode is het verzwakken van de gestelde hypothese door ook inefficiènt gedrag te beschouwen. De maximale efficiëtie, warbij de gegevens nog aan de hypothese voldoen, geeft dan een indruk van de mate warin ala deze hypothese voldaan is.

Hoewel de nadruk ligt op de ontwikkeling van niet-parametrische toetsen, wordt ruime aandacht besteed aan het afleiden van prijzen en hoeveelheden voor kapitaal woorraden. Voor het berekenen van de kiapitaalvoorraden en de schaduwprijs van kapitaal, wordt aangetoond dat de gebruikelijke anname van exponentieel verval van kapitaal op eenwoudige manier verbeterd kan worden. Beschreven wordt hoe kapitaalvoorraden gegenereend kumnen worden met behulp van een lineair dynamisch model. Hierbij is aangenomen dat de afstoot van kapitaal benaderd kan worden door een gammafunctie. Bovendien wordt beschreven hoe de schaduwprijs van kapitaal af geleid kan worden voor een willekeurig gegeven afstootfunctie van kapitaal.

\section{Generalisatie van de niet-parametrische theorie}

De niet-parametrische toetsen volgen uit theorema's, die in dit boek worden afgelleid. Deze theorema's worden bewezen doon een geschikte nuts- of produktiefunctie te construeren voor de gegeven data. Als de data aan bepaalde eigenschappen wolldoen, zoals concaviteit van de budgetrestricties, houdt dit in dat deze geconstrueerde functie bepaalde eigenschappen heeft. Dit betekent dat een groot aantal varianten mogelijk is op de theorema's, waarbij eigenschappen van de geconstrueerde functie volgen uit aannames over eigenschappen van de gegeven data. Een andere generalisatie, die vaak zonder problemen gemaakt kan worden, is de generalisatie van single-output naar het geval waar meerdere outputs zijn toegestaan.

Er worden geen differentieenbare nuts- en produktiefuncties beschouwd. In een artikel van Chiappori en Rochet (1987) staat echter een elegant bewijs, waarin een differentieerbare nutsfunctie is afgeleid als de convolutie van twee functies. Als men het bestaan van een differentieerbare nuts- of produktiefunctie wil bewijzen, kan deze methode op veel resultaten in dit boek worden toegepast.

Nu er een overzicht is van de resultaten, is het mogelijk om de verbanden tussen de verschillende niet-parametrische theorema"s te ondlerzoeken. Dit met het doel ze in te passen in een algemenere theorie. Zo' $\mathrm{n}$ theorie kan antwoord geven op de vraag voor welke specificaties er met succes een nietparametrisch theorema afgeleid kan worden. Bij de anvang van dit onderzoek was dit voornamelijk een kwestie van trial en error. Later werd duidelijk waarom bepaalde specificaties een probleem waren en andere niet. De rode draad in zo'n algemene theorie is de symmetrie wan het optimeringsprobleem. Lineair homogene kostenminimering, waarvoor de Lagrangeaan symmetrie vertoont, leverde bijwoorbeeld geen problemen op. Dit in tegenstelling tot de zwak scheidbare nutsfunctie, waarvoor de Lagrangeaan geen voor de hand liggende symmetrie vertoont.

\section{Toepassingen van de niet-parametrische theorie}

Wat zijn de toepassingen van de niet-parametrische theorie? Ten eerste kan deze theorie gebruikt worden om een overzicht te krijgen van de eigenschappen van een yerzameling gegevens. Dit kan tijd besparen bij het zoeken naar een parametrische specificatie van de nuts- of produktiefurictie. De niet- 
parametrische toetsen dienen dam als een leidraid, die aangeeft welke mogelijkheden er zijn. De niet-parametrische theorie kan verwolgens een hulpmiddel zijn bij het oplossen van theoretische problemen. Voorbeelden heryoor zin problemen warbij voor een gegeven vraagrelatie gevraagd wordt naar het bestaan van een nuts- of produktiefunctie.

Een toepasing van de niet-parametrische benadering die voorall bruikbaar is voor producentengedrag, is de woorspelling van econonisch gedrag. Dit onderwerp komt niet aari de orde in dit boek, maar het gereedschap ervoor wordt alangerelkt in de vorm van grenzen voor de produktiefunctie en grenzen voor de technische efficientie en technische vooruitgang. Deze grenzen kunnen beschouwd worden als behouden grootheden, die volgen wit de symmetrie van het optimeringsmodel, dat de waarnemingen genereert. Voor deze behouden grootheden zijn analoge voorbeelden te vinden in de fysica, zoals energie en impulsmoment, die ook daar voortkomen uit het feit dat de Lagrangeaan symmetrie vertoont. In de theoretische fysica geldt de niet-parametrische benadering; met symmetrie-veronderstellingen als uitgangspunt, als standaardgereedschap. Zo is bijvoorbeeld het bestatan van nieuwe elementaire deeltjes voorspeld. Te werwachten is dat dese niet-parametrische benadering ook wan waarde zall blijken te zijn in de economische wetenschap. 


\section{CURRICULUM VITAE}

Martijn Houtman was born on 23 june 1955 in The Hague. After finishing B.A. mathematics and physics with subsidiary subject sociology, he graduated in 1982 in mathematics with the subsidiary subjects computer science and physics. Subsequently he worked as fellow assistant at the Department of Economics of the University of Groningen. His work consisted of writing computer programs, concerning nonparametric tests of consumer behaviour. After this he was several years engaged in research at the Department of Economics of the University of Groningen. During this time he was for several months on detachment at the University of Michigan in Ann Arbor.

Besides research on nomparametric theory he studied the structure of economic models. He also made a simulation model of the Dutch economy. At the moment the is graduating in physics at the University of Leiden. His main subjects are theoretical physics and artificial intelligence. Occasionally he occupied himself with research in other areas. For example, he investigated the way foreigners are presented at the Dutch television. Since 1995 he works parttime as a researcher at the University of Limburg in Maastricht. 